\title{
La Représentation du local, de l'universel et de la réalité dans Rue la Poudrière, Le Voile de Draupadi et Le Sari vert d'Ananda Devi
}

\author{
by \\ MUKTA DAUSOA
}

A thesis

submitted to the Victoria University of Wellington in fulfillment of the requirements for the degree of

Doctor of Philosophy

Supervisors

Associate Professor Jean Anderson

Dr Myreille Pawliez

Victoria University of Wellington

2015 


\begin{abstract}
This doctoral thesis, written in French and entitled «La Représentation du local, de l'universel et de la réalité dans Rue la Poudrière, Le Voile de Draupadi et Le Sari vert d'Ananda Devi », is an in-depth historico-sociological analysis of Mauritian writer Ananda Devi's three novels published in 1988, 1993 and 2009 respectively. Having Mauritius as their background, these three novels introduce themes such as slavery, the trans-oceanic experience of Indian indentured labourers, prostitution, rape, domestic violence and homicide, through female protagonists in Rue la Poudrière and Le Voile de Draupadi, and a male protagonist in Le Sari vert.

Existing research on Devi's work concentrates mostly on the plight of women, who are victims of a Mauritian patriarchal society. Moving away from this approach, my research focuses on the spatial, historical and sociological dimensions in order to closely analyse the surroundings of the characters. The first focus of this research is to see whether these three novels exclusively allude to Mauritian society or also deal with universal concepts. The second focus is to determine the degree of realism of these three fictional works. Thus, the overall focus of this research is to scrutinise the degree of particularism, universalisation and realism in the geographical, historical and social dimensions of the novels.

I begin with a brief presentation of Ananda Devi and her place in Mauritian literature. Then, I explain the objectives of the thesis and introduce my methodologies, which include the theories of Tzvetan Todorov and Jean-Marc Moura used to analyse the local and universal aspects, and the theories of Guy de Maupassant, Roland Barthes, Maureen Ramsden, Vincent Jouve, Michael Riffaterre, Mark Sainsbury, Névine El Nossery used to frame the examination of realism. Sociological theories of Emile Durkheim and Robert Merton are also elaborated, to support the analysis of the social dimension. In order to better scrutinise the historical dimension of the novels, I have used the work of Mauritian and international historians as well as Mauritian and international governmental reports. Khal Torabully's notion of coolitude is also discussed to evaluate how different ethnic groups are represented in the novels. I have in addition coined the term "le rêve mauricien", in relation to the social dimension of the novels. Finally, I conclude with issues of realism, the local, the universal and fiction.
\end{abstract}




\section{RÉSUMÉ}

Cette thèse de doctorat, intitulée «La Représentation du local, de l'universel et de la réalité dans Rue la Poudrière, Le Voile de Draupadi et Le Sari vert d'Ananda Devi » est une analyse historico-sociologique approfondie de trois romans parus en 1988, 1993 et 2009, de l'auteure mauricienne Ananda Devi. Ayant pour cadre l'île Maurice, ces romans présentent des thèmes comme l'esclavage, le sort des travailleurs engagés indiens, la prostitution, le viol conjugal, la violence domestique et l'homicide, à travers des protagonistes féminins pour Rue la Poudrière et Le Voile de Draupadi, et masculins dans Le Sari vert.

Les travaux de recherche existants sur Ananda Devi se concentrent principalement sur le sort des femmes, sous le joug de la société mauricienne patriarcale. Se détachant de cette approche essentiellement féministe, nous étudions les dimensions spatiale, temporelle et sociale des trois romans pour analyser l'univers et la situation sociale des personnages. Le premier objectif de ce travail est de considérer si ces œuvres dépeignent exclusivement la société mauricienne, ou s'ils couvrent plutôt des questions plus étendues. Le deuxième axe de la recherche consiste à analyser leur degré de réalisme autant au niveau spatial et temporel que sociologique. Ainsi, il est essentiellement question de déterminer la part du réalisme, de l'universalisme et du particularisme dans ces trois romans.

Nous recensons d'abord les écrits d'Ananda Devi et explorons sa place dans la littérature mauricienne, avant d'expliquer l'objectif et la méthodologie de la thèse. Tout particulièrement, nous faisons appel aux travaux de Tzvetan Todorov et Jean-Marc Moura pour étudier les concepts de local et d'universel, et ceux de Guy de Maupassant, Roland Barthes, Maureen Ramsden, Vincent Jouve, Michael Riffaterre, Mark Sainsbury, Névine El Nossery pour analyser le réalisme. Nous avons également recours aux théories sociologiques d'Emile Durkheim et de Robert Merton pour l'analyse de la dimension sociale. Nous utilisons aussi les conclusions d'historiens et d'analystes gouvernementaux, mauriciens et internationaux, pour appuyer notre discussion. Nous nous intéressons de plus à la notion de coolitude de Khal Torabully pour mieux appréhender la situation des différents groupes ethniques dont il est question dans les romans. Nous élaborons un nouveau concept, celui du "rêve mauricien", qui nous permettra de réfléchir sur la dimension sociale. Nous terminons en concluant sur les questions de réalisme, local, universel et fiction. 


\section{ACKNOWLEDGEMENTS}

I am grateful to Victoria University of Wellington for providing me with research grants to travel to Mauritius in 2012 and 2013 and for financial support in 2013 and 2014. I wish to express my sincere appreciation to Dr Marco Sonzogni and Dr Limin Bai for their support and assistance with administrative formalities.

I wish to express my heartfelt gratitude to my supervisors for their support and belief in me. I thank Dr Myreille Pawliez for her availability, moral support, perseverance and continuous encouragement, which always gave me a boost, and Associate Professor Jean Anderson for her insightful comments, enlightening suggestions and experience which guided and helped me be more critical towards my work.

I would especially like to draw attention to the encouragement, understanding and consideration of my parents Rajnarain and Meerah Guttee, which enabled me to always keep going in difficult times and reach my goal. They taught me that determination, perseverance and discipline are the keys to success. I pay a deep tribute to my mother, who sacrificed her career for her children. I also benefited greatly from the encouragement of my in-laws, Dhyaneswar and Jyoti, not forgetting my sister Geetanjalee and brother Divesh, who always stood by me throughout this project. I am most grateful to Sunita and Deo Narayan who have been my mentors since my arrival in New Zealand. Without their help, I would never have completed this project successfully. I deeply regret that my maternal grandfather Bharut Jingree, who was a firm believer in girls' education, did not live long enough to see my project completed. I also thank my colleagues Janette Briggs for her sense of humour and moral support, Alessandro Macilenti for sharing stimulating discussions, Loveday Kempthorne for all her encouragement and advice and the administrative staff, Sarah Walton, for her immense help and expertise in formatting the thesis.

I convey my sincere thanks to my son Mrinal for bringing great joy to my life, for giving me the happiness of being a mother and much love after long and tiring hours of research. Last, but not least, I am particularly indebted to my husband Mahendra, who enabled me to grow, push my limits and fulfill my dreams by always supporting, guiding and assisting me, even in most difficult times. This thesis would never have been completed without his sacrifices, patience, determination and unconditional love. I dedicate this thesis to him. 


\section{REMERCIEMENTS}

Je suis reconnaissante à l'université Victoria de Wellington pour m'avoir accordé une aide financière en 2012 et une subvention en 2013 pour conduire des recherches à Maurice. Je remercie aussi l'université pour m'avoir exemptée des frais d'inscription en 2013 et 2014. J'exprime aussi ma reconnaissance à Marco Sonzogni et Limin Bai, qui m'ont toujours épaulée et aidée dans ces démarches administratives.

Je tiens à exprimer ma profonde gratitude à mes directrices de thèse pour avoir cru en moi et pour m'avoir soutenue jusqu'au bout. Je sais gré à Myreille Pawliez pour sa disponibilité, son soutien moral, sa persévérance et ses encouragements inlassables qui m’ont stimulée et à Jean Anderson pour ses remarques percutantes, ses suggestions éclairantes et son expérience qui m'ont guidée à devenir plus critique envers mon travail.

Il me faut aussi souligner les encouragements, la compréhension et les attentions de mes parents Rajnarain et Meerah Guttee, qui m'ont permis de persévérer dans les moments difficiles et de réaliser mon rêve. Ils m'ont appris que la détermination, la persévérance et la discipline sont la clé du succès. Je rends hommage à ma mère qui a sacrifié sa carrière pour ses enfants. J'ai aussi bénéficié des encouragements de mes beaux-parents, Dhyaneswar et Jyoti, sans oublier ma sœur et mon frère Geetanjalee et Divesh, qui m'ont épaulée tout au long de ce projet. Sunita et Deo Narayan m'ont aidée depuis mon arrivée en Nouvelle-Zélande : sans leur aide, je n'aurais jamais pu mener à bien ce projet. Je regrette profondément que mon grandpère maternel, Bharut Jingree, qui croyait avec ferveur à l'éducation des filles, n'ait pas vécu assez longtemps pour célébrer l'aboutissement de cette thèse. Je remercie aussi mes collègues, Janette Briggs pour son sens de l'humour et son soutien moral, Alessandro Macilenti pour ses discussions très stimulantes, Loveday Kempthorne pour ses encouragements et conseils et le personnel administratif, Sarah Walton, pour son immense aide et expertise au formatage de la thèse.

Un grand merci à mon fils Mrinal, pour me rappeler constamment la joie de vivre et me faire connaître les bonheurs d'être mère et surtout pour m'avoir apporté beaucoup d'amour après mes longues journées de travail. Enfin, je suis particulièrement redevable à mon époux, Mahendra, qui m'a permis de m'épanouir, de repousser mes limites, de réaliser mon rêve le plus cher, et qui m'a soutenue, guidée, épaulée avec sa conviction infaillible. Ce travail n'aurait jamais pu être achevé sans ses 
sacrifices, sa patience, sa détermination et son amour inconditionnel. Je lui dédie cette thèse. 


\section{TABLE DES MATIÈRES}

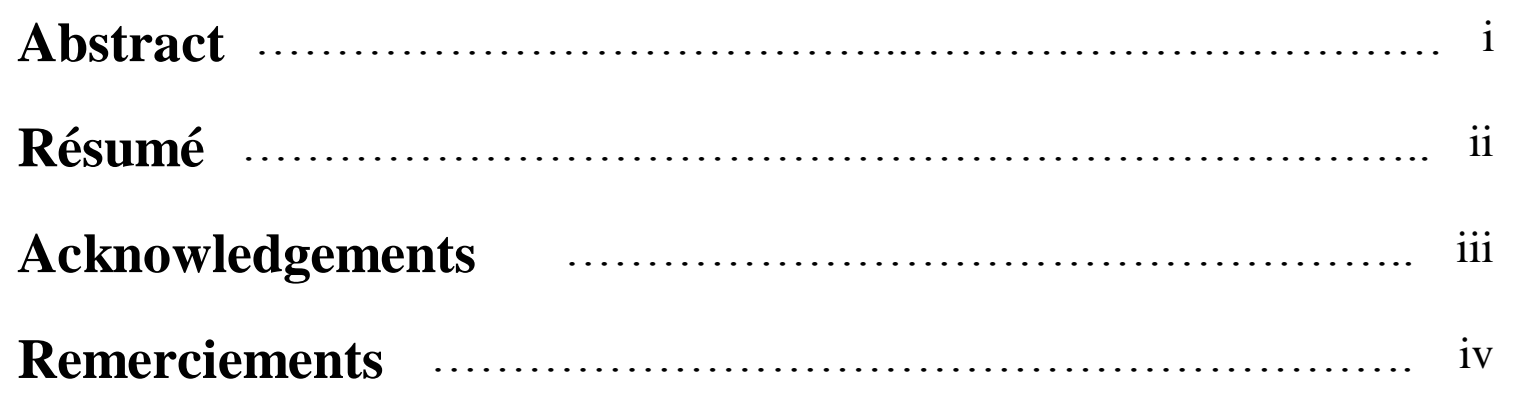

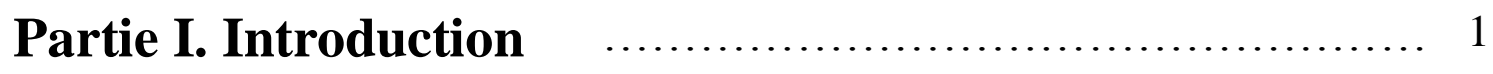

L'auteure et sa place dans la littérature mauricienne $\ldots . \ldots \ldots \ldots \ldots .2$

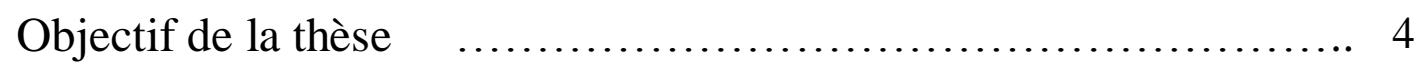

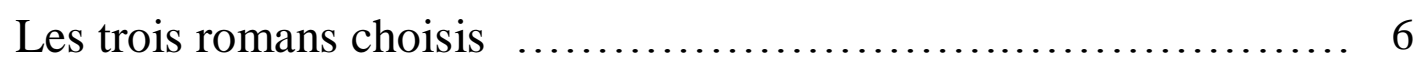

Bilan des recherches sur les trois roman $\quad \ldots \ldots \ldots \ldots \ldots \ldots \ldots \ldots . \ldots \ldots$

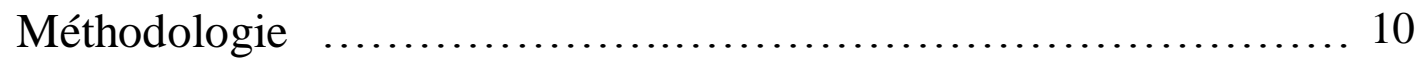

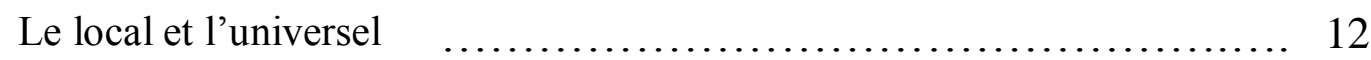

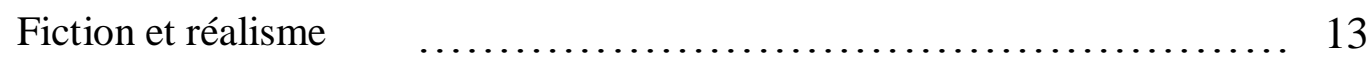

La microsociologie $\quad$...................................... 15

La coolitude $\quad$............................................. 18

Le rêve mauricien $\quad$.......................................... 19

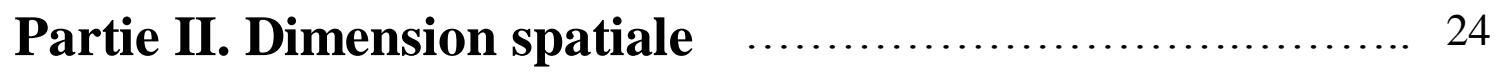

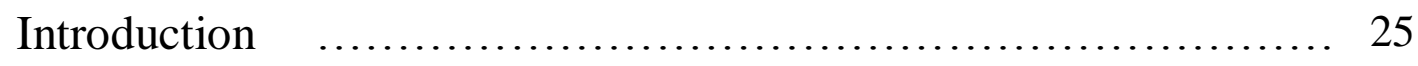

Rue la Poudrière $\quad$........................................... 25

Géographie et toponymie dans Rue la Poudrière $\quad$.................. 27

Flore et faune dans Rue la Poudrière $\quad$................................ 27

Une description spatiale à la fois locale et universelle dans Rue la

Poudrière 
Géographie et toponymie dans Le Voile de Draupadi $\quad$..................... 35

Nature, flore et faune dans Le Voile de Draupadi ...................... 38

Un décor local et réaliste dans Le Voile de Draupadi $\quad$................ 42

Le Sari vert $\quad$.................................................. 42

Géographie et toponymie dans Le Sari vert $\quad$.......................... 43

Flore et faune dans Le Sari vert $\quad$.................................... 44

Une localisation locale mais une faune et flore universelle dans

Le Sari vert

Localisation spécifique et une universalisation dans les trois romans 46

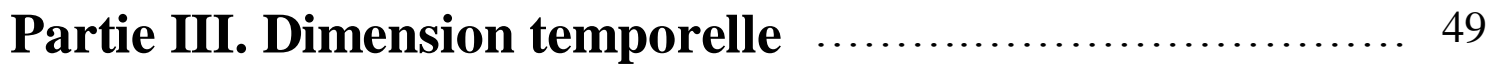

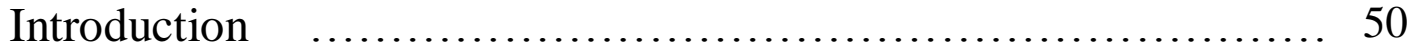

Rue la Poudrière $\quad$................................................. 51

L'ancrage de l'histoire $\quad$....................................... 51

La grève des dockers de $1979 \quad$.................................. 52

La destruction des bâtiments à la Butte ............................. 54

Un ancrage et un passé local dans Rue la Poudrière $\quad$................. 56

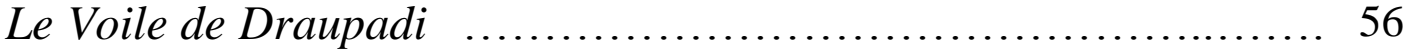

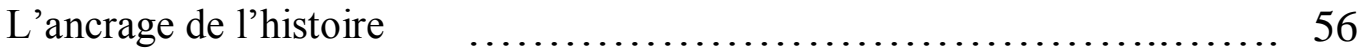

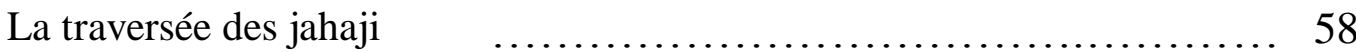

bhai

Les travailleurs engagés une fois à l'île Maurice $\quad \ldots \ldots \ldots \ldots \ldots \ldots \ldots . . . \ldots 60$

Un ancrage local et réaliste et un passé à la fois local et universel dans

Le Voile de Draupadi 
L'ancrage de l'histoire

La falaise du Morne, les esclaves africains et malgaches à l'île Maurice ... 65

Les émeutes de juillet 1937 dans les établissements sucriers à l'île

Maurice

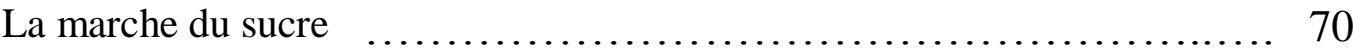

Le conflit racial de janvier 1968 à l'île Maurice $\quad$....................... 70

Anachronisme du présent diégétique et faits historiques à la fois locaux et universels dans Le Sari vert

Cadre réel local et problèmes universels dans les trois romans

Partie IV. Dimension sociale

Introduction

Rue la Poudrière

La communauté créole dans Rue la Poudrière

La classe inférieure dans Rue la Poudrière

Utilisation des langues dans Rue la Poudrière

Relations de couple dans Rue la Poudrière

Marie et Edouard

Paule et Mallacre

Paule et Tapsy

Le rêve mauricien dans Rue la Poudrière

Représentation réaliste mais universelle de la classe ouvrière dans

Rue la Poudrière

Le Voile de Draupadi

Représentation des ethnies dans Le Voile de Draupadi 
Les disparités raciales et discriminatoires entre la communautés

hindoue et créole dans Le Voile de Draupadi

La représentation de la classe sociale dans Le Voile de Draupadi

La classe aisée dans Le Voile de Draupadi

La classe inférieure dans Le Voile de Draupadi

Le registre de langue utilisé dans Le Voile de Draupadi

Le rêve mauricien dans Le Voile de Draupadi

Relations de couple dans Le Voile de Draupadi

Yashoda et son mari

Anjali et Dev

Margaret et Shyam

Représentation réaliste à la fois locale et universelle des ethnies et classes sociales dans Le Voile de Draupadi

La représentation des différentes ethnies dans Le Sari vert

La communauté hindoue dans Le Sari vert

La communauté créole dans Le Sari vert

Les disparités raciales et discriminatoires dans Le Sari vert

La classe sociale et le registre de langue dans Le Sari vert

Le rêve mauricien dans Le Sari vert

Relations de couples dans Le Sari vert

Le couple Bissam

Kitty et son mari

Représentation réaliste à la fois locale et universelle des ethnies et classes sociales dans Le Sari vert 
Partie V. Du local à l'universel : trois romans réalistes $\quad \ldots \quad 142$

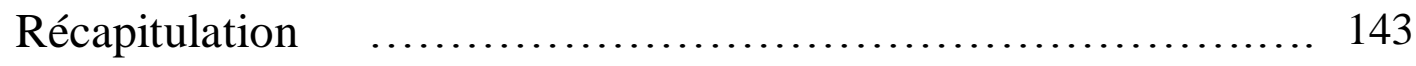

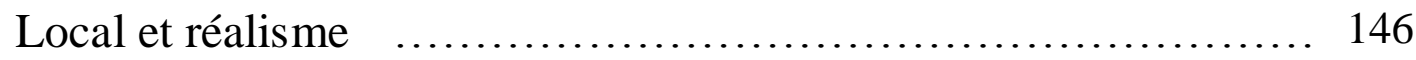

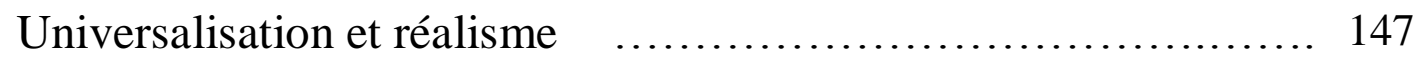

Le factuel et le fictionnel $:$ le «mensonge du romancier $» \ldots . . . . .149$

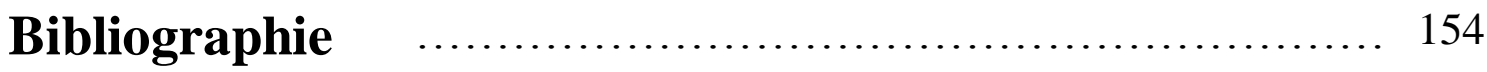




\section{Partie I}

\section{Introduction}




\section{L'auteure et sa place dans la littérature mauricienne}

Née à l'île Maurice, à Trois-Boutiques, le 23 mars 1957, Ananda Devi Nirsimloo-Anenden est écrivaine, ethnologue et traductrice à l'Organisation mondiale de la propriété intellectuelle (OMPI). Cette auteure mauricienne, qui a longtemps vécu en Afrique, habite désormais à Ferney-Voltaire, tout près de Genève. Elle écrit principalement en français mais ses romans contiennent aussi des phrases et dialogues en hindi, en bhojpuri et en créole mauricien. Reconnue comme « la grande dame des lettres mauriciennes ${ }^{1}$, elle continue à écrire et revient souvent dans son île natale pour retrouver sa source d'inspiration.

L'auteure a publié en 1973, à l'âge de quinze ans, une nouvelle, La Cité Atlee, pour laquelle elle a reçu le prix du concours d'écriture de l'Office de radiodiffusion télévision française. Quatre ans plus tard, elle a publié Solstices, un recueil de nouvelles qui l'a rendue célèbre à Maurice. Ananda Devi a aussi soutenu, plus tard, un doctorat d'anthropologie sociale tout en continuant à s'adonner à l'écriture. C'est ainsi qu'elle a publié en 1987, après une absence de dix ans de la scène littéraire, un deuxième recueil de nouvelles, Le Poids des êtres. En 1988, Devi a publié son premier roman, Rue la Poudrière, aux Nouvelles Editions Africaines, à Congo-Brazzaville, où elle habitait. On lui doit, depuis, de nombreux autres romans parus dans différentes maisons d'édition, telles que Dapper, L'Harmattan et plus récemment, Gallimard. Ainsi, elle a écrit Le Voile de Draupadi (1993), L'Arbre-fouet (1997), Moi, l'interdite (2001) qui a remporté le prix Radio France du livre de l'océan Indien, Pagli (2001), Soupir (2002), La Vie de Joséphin le Fou (2003), Eve de ses décombres (2006), couronné par le prix des Cinq Continents de la Francophonie et le prix du livre RFO, Indian Tango (2007) qui a décroché le prix France Télévision, Le Sari vert (2009) couronné par le prix LouisGuilloux, son premier récit autobiographique, Les Hommes qui me parlent (2011) et Les Jours vivants (2013). En 2010, elle a été nommée Chevalier des Arts et des Lettres. Devi est aussi l'auteure d'autres recueils de nouvelles et de poèmes. Par ailleurs, une des nouvelles de Solstices a été adaptée au cinéma par son mari et son fils en 2006 sous le titre La Cathédrale. Ils ont aussi réalisé en 2012 le film Les Enfants de Troumaron, basé sur son roman Eve de ses décombres. Son roman Pagli a été traduit en anglais par Devi elle-même et en espagnol par Manuel Serrat en 2002. Soupir a aussi été traduit en

\footnotetext{
${ }^{1}$ Fabien Mollon, «Ananda Devi : il y a une violence latente à Maurice », http://www.jeuneafrique.com/Article/JA2726p082-083.xml0/, consulté le 22 mai 2013.
} 
espagnol en 2004 par Isabel Olid, Moi, l'interdite a été traduit en hindi en 2006 par Asha Pandeet et Indian Tango a été traduit en anglais en 2011 par Jean Anderson.

A l'instar de Marcel Cabon, Carl de Souza, Nathacha Appanah et Shenaz Patel, tous originaires de l'île Maurice, Ananda Devi est incontestablement une écrivaine reconnue sur le plan international. Qualifiée comme «prolifique et talentueuse auteure mauricienne $»^{2}$, Devi «est devenue une référence dans la culture mauricienne $»^{3}$ et « suscite un intérêt grandissant dans le monde francophone » ${ }^{4}$. Aussi s'est-elle « révélée une personnalité au talent littéraire incontesté » ${ }^{5}$ et «une des romancières les plus en vue de l'océan Indien $»^{6} . \mathrm{La}$ « femme de lettres mauricienne $»^{7}$, s'est incontestablement fait une place bien à elle parmi les auteurs francophones non seulement mauriciens mais aussi internationaux.

Les romans de Devi décrivent, pour la plupart, la condition de la femme mauricienne sous le joug d'une société patriarcale. Véronique Bragard, écrivaine et conférencière à l'Université catholique de Louvain en Belgique, écrit à ce propos que l'auteure «explore avec une grande acuité de nombreux caractères humains, recomposant ainsi les multiples univers qui se côtoient, s'affrontent, se déchirent dans un espace insulaire qui n'est pas moins analysé que recréé » ${ }^{8}$. En septembre 2006, à la remise du prix des Cinq Continents de la Francophonie, le président du jury Abdou Diouf a rendu hommage à sa «belle écriture représentative de la diversité des cultures dans l'espace francophone ainsi [qu'à] l'originalité de ses personnages qui vont aux lisières de l'animalité afin de remuer la conscience de ceux qui pensent représenter l'humanité $»^{9}$. En outre, en 2009, dans le quotidien mauricien, Le Matinal, un

\footnotetext{
2 Anonyme, « Ananda Devi lauréate 2010 du prix Louis-Guilloux », http://www.lexpress.mu/article/ananda-devi-laur\%C3\%A9ate-2010-du-\%C2\%AB-prix-louis-guilloux$\%$ C2\%BB, consulté le 20 octobre 2011.

${ }^{3}$ Ibid.

${ }^{4}$ Ashwiny Kistnareddy, «Entre nomadisme et sédentarité : l'identité îlienne dans Pagli d'Ananda Devi », Le Panoptique : perspectives sur les enjeux contemporains, 2008, http://www.lepanoptique.com/sections/arts-litterature/entre-nomadisme-et-sedentarite-lidentite-iliennedans-pagli-dananda-devi/, consulté le 2 mai 2012.

${ }^{5}$ Anonyme, «Ananda Devi : prix Louis Guilloux 2010 », http://cibamike.wordpress.com/2010/08/, consulté le 17 janvier 2013.

${ }^{6}$ Anonyme, «Union des femmes de la Martinique », http://www.unionfemmesmartinique.com/?article683-textes-et-poemes-pour-la-journee-du-25-nov-a-fort-de-france, consulté le 5 décembre 2012.

${ }^{7}$ Anonyme, «Intertitres. L'écriture de fil en aiguille », http://cibamike.wordpress.com/2010/08/, consulté le 17 janvier 2013.

${ }^{8}$ Véronique Bragard, «Ananda Devi », 2001, http://www.lehman.cuny.edu/ile.en.ile/paroles/devi.html, consulté le 2 mai 2012.

${ }^{9}$ Jean-Marc Poché, « Le prix des Cinq Continents de la Francophonie à une Mauricienne », http://www.radiomoris.com/forum/nouvelles-de-lile-maurice/4382-le-prix-des-cinq-continents-de-lafrancophonie-une-mauricienne.html, consulté le 25 juillet 2011.
} 
journaliste présente Ananda Devi comme «le porte-parole de toutes ces femmes meurtries, ici et ailleurs, sous toutes les latitudes ${ }^{10}$ et précise «alors que d'autres récusent l'île, Ananda Devi y retourne, à chaque fois qu'elle prend sa plume pour créer un nouveau personnage $»^{11}$.

\section{Objectif de la thèse}

Il n'est guère surprenant que les œuvres de Devi aient déjà fait l'objet d'articles, de chapitres de livres et de thèses ${ }^{12}$ et nombreux sont ceux qui ont tenu des entretiens avec l'auteure ${ }^{13}$. Ces nombreux travaux ${ }^{14}$ considèrent, pour la plupart, les thèmes souvent abordés par Devi tels le silence des femmes ${ }^{15}$, l'amnésie ${ }^{16}$, l'anorexie ${ }^{17}$, la folie $^{18}$, la métamorphose ${ }^{19}$, la violence infligée aux femmes ${ }^{20}$, la rébellion de la femme ${ }^{21}$ ou les conditions inhumaines des travailleurs engagés ${ }^{22}$. Si tous ces articles et

\footnotetext{
${ }^{10}$ Anonyme, «Le Sari vert d'Ananda Devi : une constance littéraire qui force l'admiration », Le Matinal, 2009, http://www.lematinal.com/mobile/magazine/268-sari-vert-dAnanda-Devi-une-constance-littrairequi-force-ladmiration.html, consulté le 2 mai 2012.

${ }^{11}$ Ibid.

${ }^{12}$ Voir la section «Thèses consacrées aux romans d'Ananda Devi » de la bibliographie pour la liste exhaustive.

${ }^{13}$ Voir la section « Entretiens d'Ananda Devi consultés » de la bibliographie.

${ }^{14}$ Voir la section «Critiques, articles, ouvrages et sites relatifs à Ananda Devi » de la bibliographie pour la liste exhaustive.

${ }^{15}$ Véronique Bragard, «Cris des femmes maudites, brûlures du silence, la symbolique des éléments fondamentaux dans l'œuvre d'Ananda Devi », Notre librairie, 142, 2000, p. 66-73; Florina-Liliana Mihalovici, "La "guerre silencieuse" de la femme dans une société patriarcale qui la dévore : Le Sari vert d'Ananda Devi », dans Parole au silence, Limoges, Pulim, 2012, p. 103-113.

${ }^{16}$ Amaleena Damlé, «Phantasmal Relics, Psychoanalytical and Deconstructive Ghosts in Moi, l'interdite and Pagli, Amnesia and Haunting », Amnesia: Private and Public Memory in Modern French Culture, Peter Collier, Anna Magdalena Elsner, Olga Smith, eds, Bern, Peter Lang, 2009, p. 229-240.

${ }^{17}$ Njeri Githire, « The Semiotics of (Not) Eating: Fasting, Anorexia, and Hunger Strike in Ananda Devi's Le Voile de Draupadi», Nottingham French Studies, 48, 1, 2009, p. 82-93.

${ }^{18}$ Ashwiny Kistnareddy, «Interrogating Identity: Psychological Dislocations in Ananda Devi's Novels », Dalhousie French Studies, 94, Spring 2011, p. 27-38 ; Putu Basey Itsieki, Jean de Dieu, « Amour, folie, liberté : la dissidence féminine dans l'écriture d'Ananda Devi », La Tortue verte : revue en ligne des littératures francophones, http://w3.gril.univ-tlse2.fr/analyses/A2012/ITSIEKI.pdf, consulté le 25 janvier 2013.

${ }^{19}$ Magali Marson, «Carnalité et métamorphoses chez Ananda Devi », Indispensables animaux, Notre librairie 163, 2006, p. 71-76 ; Serge Meitinger, «L'Innocence meurtrière : humanité et animalité dans les récits d'Ananda Devi », Francofonia, 17, 2008, p. 161-178; Serge Meitinger, «Les fantastiques dans les nouvelles d'Ananda Devi », Iris : les cahiers du Gerf, 26, 2004, p. 17- 28.

${ }^{20}$ Karine Bénac-Giroux, «Le martyre des femmes dans Le Sari vert d'Ananda Devi », dans Libertés et Oppressions, Paris, L’Harmattan, p. 103-113 ; Julia Effertz, « Le Prédateur, c'est moi : l'écriture de la terre et la violence féminine dans l'œuvre d'Ananda Devi», Violence in French and Francophone Literature and Film, James Day, Amsterdam, Rodopi, 2008, p. 71-82.

${ }^{21}$ Rohini Bannerjee, «Daughter Forsaken: la résistance of the Indo-Mauritian Girl Child in Ananda Devi's Novel », dans India in the World, Cristina M. Gámez-Fernández, Antonia Navarro-Tejero, Cambridge Scholars Press, 2011, p. 123-134 ; Véronique Bragard, «Eaux obscures du souvenir. Femme et mémoire dans l'œuvre d'Ananda Devi », Convergences and Interferences: Newness in Intercultural Practices. Ecrire d'une nouvelle ere/aire, Amsterdam et New York, Rodopi, 8, 2001, p. 187-199 ; Bruno Cunniah, «La textualisation de la dissidence chez Ananda Devi et Shakuntala Boolel », Les Cahiers du GRELCEF, 3, 2012, p. 123-137; Valérie Magdelaine-Andrianjafitrimo, «De Sita à Draupadi. Les
} 
travaux réalisés éclairent l'œuvre de Devi en dégageant ses thèmes et en examinant le style de l'auteure, nous proposons d'étudier la dimension spatiale, l'ancrage temporel et la dimension sociale de trois de ses romans pour tenter de distinguer à quel point ces aspects peuvent être décrits comme réalistes. L'autre but de cette recherche sera de percevoir dans quelle mesure la représentation de ces aspects est spécifique à l'île Maurice et à la société mauricienne.

Nous tenterons donc d'analyser comment dans les trois romans, qui ont pour décor l'île Maurice, l'auteure fluctue entre deux extrémités : le local et l'universel, la fiction et la réalité. Même si l'auteure soutient que le cadre de ses romans est imaginaire, les lieux et certains événements touchent de près la société de l'île Maurice, ainsi que d'autres sociétés ailleurs. Nous notons, ici, une contradiction entre ce qu'affirme l'auteure et le fait que ses écrits soient inspirés par sa connaissance approfondie de l'île Maurice. Nous nous interrogerons sur cette contradiction apparente en suivant les deux axes qui sous-tendent les romans de Devi. Si le roman est, certes un récit imaginaire, il repose en général sur un fond réel. Devi brouille ainsi les barrières entre la fiction et la réalité, entre le local et l'universel. C'est d'ailleurs pourquoi elle atteint un lectorat très large et varié qui arrive à non seulement cerner la portée universelle de ses romans, mais aussi à déchiffrer son caractère local.

Pour notre étude, nous avons sélectionné Rue la Poudrière, Le Voile de Draupadi et Le Sari vert parce qu'ils ont comme toile de fond l'île Maurice et présentent des personnages issus de classes sociales diverses qui évoluent dans un univers mauricien et sont témoins de réalités historiques qui ont marqué le passé de l'île. Nous en explorerons la toponymie et la topographie, la flore et la faune, les références historiques, la situation et les conditions de vie des personnages afin de déterminer dans quelle mesure ces récits représentent la réalité mauricienne. Nous nous efforcerons aussi de démontrer à quel point cette réalité peut être universelle et toucher d'autres sociétés. Nous visons ainsi à mesurer le degré d'universalisme et de particularisme de ces romans.

ambivalences d'Anjali et de Vasanti dans Le Voile de Draupadi, d'Ananda Devi », La Revue «Vues d'ailleurs », 6, 2011-2012, p. 1-25.

${ }^{22}$ Rohini Bannerjee, «The Kala Pani Connection: Francophone Migration Narratives in the Caribbean Writing of Raphaël Confiant and the Mauritian Writing of Ananda Devi », Anthurium: a Caribbean Studies Journal, 7, 2009, p. 1-11 ; Véronique Bragard, «Regards croisés sur la mémoire coolie des Antilles aux Mascareignes », Nouvelles études francophones, 21, 2006, p. 163-180. 


\section{Les trois romans choisis}

Rue la Poudrière, le premier roman d'Ananda Devi paru en 1988, est narré à la première personne et se déroule dans un cadre tout à fait mauricien. Il raconte l'histoire de la petite Paule, fille indésirée du couple Marie et Edouard qui habitent dans un quartier très pauvre de Port-Louis, la capitale de l'île Maurice. Paule passe ainsi sa petite enfance dans un quartier non spécifié de Port-Louis, puis à presque «trois ou quatre ans $»^{23}$ ou bien à «six ans ${ }^{24}$ [l'âge de Paule, ici, reste flou] va habiter aux Dockers' Flats et à La Butte. Finalement, elle passe plusieurs années de sa jeunesse au centre de la capitale, dans la rue la Poudrière. Paule vit dans une famille très pauvre. Son père Edouard travaille au port en tant que débardeur, alors que sa mère, Marie, exerce plusieurs petits métiers. Cette dernière prétend être guérisseuse et pratique la magie noire. Edouard, alcoolique, et Marie, rancunière, échangent des injures, des coups de pied et de poing qui font partie de leur quotidien et de celui de Paule. Après un accident de travail, Edouard devient chômeur et alcoolique. Un jour de délire éthylique, il vend Paule à son ami Mallacre, proxénète de la rue la Poudrière. Paule fait alors la connaissance d'Aline, une jeune fille de dix-sept ans qui habite aussi la maison close. Les deux jeunes filles se lient d'amitié mais Aline met fin à ses jours après une histoire d'amour malheureuse. Très vite initiée à la prostitution, par Mallacre, Paule fait la connaissance de Tapsy, son premier client qui devient son petit ami. Paule est progressivement envahie par le désir d'avoir un enfant qu'elle pourrait aimer sans limites. Se croyant enceinte, elle ne sait pas si c'est de Mallacre, de Tapsy ou de son dernier client, un ivrogne, qui s'est précipité sur elle dans l'obscurité de sa minuscule chambre de la maison close. Or, Paule a reconnu ce client à sa voix et à sa silhouette vue au clair de lune : c'est son père, Edouard. Dégoûtée et outrée, Paule se sauve de la maison close et erre dans les rues de Port-Louis jusqu'à ce qu'elle arrive à son ancienne demeure de la Butte où elle boit une des tisanes que préparait autrefois Marie, et qui s'avère être du poison. Agée d'une vingtaine d'années, Paule meurt dans cette vieille baraque alors qu'à l'extérieur, des bulldozers arrivent pour raser le quartier déjà en ruines.

Le Voile de Draupadi, publié en 1993, est l'histoire d'Anjali, l'épouse de Dev, un avocat, et aussi mère de Wynn, leur fils unique, qui est en train de mourir d'une forme de méningite aiguë. Alors qu'elle veille son fils, Anjali, aussi narratrice, se

\footnotetext{
${ }^{23}$ Ananda Devi, Rue la Poudrière, Abidjan, Nouvelles Editions Africaines, 1988, p. 11.

${ }^{24}$ Ibid., p. 9.
} 
rappelle certains épisodes de son enfance passés avec sa cousine Vasanti qui s'était accidentellement immolée le jour d'une cérémonie hindoue, sous les yeux d'Anjali et de son frère Shyam. Vasanti était très belle mais très différente des autres filles de son âge et sa mort avait traumatisé Anjali. Pour sauver la vie de Wynn, Dev demande à sa femme de faire le sacrifice de la marche sur le feu. Malgré sa peur, Anjali cède aux pressions de son mari et de sa belle-famille et accepte de faire une période de jeûne et de prières pour demander la grâce de Dieu. Si le rituel de la marche sur le feu n'aide en rien à sauver la vie de Wynn, il permet à Anjali de se retrouver, et surtout de prendre sa vie en main. Dévastée par la mort de son fils et dégoûtée par le comportement de son mari, Anjali sollicite le divorce. Elle se retrouve seule, mais il lui reste son amie Fatmah.

Le Sari vert, publié en 2009, est le quatorzième livre de l'auteure et relate l'histoire d'un vieil homme malade et alité. Médecin de profession, le docteur Bissam Sobnath était autrefois connu sous le nom de «Dokter-Dieu». C'est lui, la voix principale qui livre, au fur et à mesure, le secret qu'il porte, en une sorte de monologue intérieur. Les événements sont donc perçus à travers le regard qu'il porte sur les trois femmes importantes de sa vie : sa femme, sa fille Kitty, et sa petite-fille Malika. Ce médecin hindou s'était marié à une belle jeune fille âgée de quinze ans qui meurt à vingt ans dans des circonstances troubles. Le nom de cette jeune femme n'est jamais mentionné mais elle est définie par son sari vert. Le couple avait eu deux enfants, Kitty et un fils décédé quelques jours après sa naissance. Plus tard, Kitty se marie à un libraire et ce couple aura, à son tour, une fille, Malika. Après la mort soudaine du gendre, Kitty et Malika vont habiter chez le vieil homme pendant un certain temps. Malika devient plus tard institutrice et son grand-père désapprouve sa relation avec sa compagne, Marie-Rose. Décidées de résoudre le mystère de la mort de leur mère et grand-mère, Kitty et Malika essayent de faire parler le vieil homme mais celui-ci meurt et le doute persiste. 


\title{
Bilan des recherches sur les trois romans
}

Il est à noter que Le Voile de Draupadi et Le Sari vert ont été l'objet de plusieurs critiques ou analyses alors que Rue la Poudrière n'a presque pas reçu de critiques, peut-être, parce que Rue la Poudrière a été publié par une petite maison d'édition africaine à une époque où l'auteure n'était pas encore connue. Cette idée est aussi partagée par Nivoelisoa Galibert, qui avance que :

\begin{abstract}
Rue la Poudrière trop lointaine pour être de l'histoire immédiate, est de fait paradoxalement trop proche pour entrer dans le cadre de l'histoire contemporaine. La documentation apportée est le plus souvent de seconde main, ou fondée sur des dires de tiers. Ouvrage de souvenirs et d'émotions toutefois, Rue la Poudrière peut rester dans nos archives ${ }^{25}$.
\end{abstract}

Parmi les rares articles consacrés à ce roman celui de Françoise Lionnet souligne que Rue la Poudrière résiste aux analyses critiques qui tentent de le domestiquer, de le catégoriser ou de l'immobiliser dans un cadre littéraire ou culturel rigide $^{26}$. Toutefois, Cooper retient que le roman est présenté comme un feu brûlant d'introspection, un voyage intérieur passionné, la quête mystique d'un "soi" privé de toute liberté de choix, un "soi” qui n'a en fait peut-être jamais existé ${ }^{27}$. Cooper estime que le roman forme une épopée mystique ${ }^{28}$. D’après Vicram Ramharai, « Ananda Devi, dans Rue la Poudrière dévoile un aspect de Port-Louis que les auteurs contemporains tendent à occulter ou effleurer seulement $»^{29}$. Il est d'avis que ce roman «pose [...] le problème de la relation entre le "soi" et la société, entre le moi et les espaces liés à des

\footnotetext{
${ }^{25}$ Nivoelisoa Galibert, «Port de badge et vision panoptique : les Dockers' Flats de Port-Louis dans Rue la Poudrière (1988) d'Ananda Devi », Revue historique des Mascareignes, 5, 2004, p. 97.

${ }^{26}$ "Rue la Poudrière escapes all critical attempts to domesticate, label, or immobilize it within an unambiguous literary or cultural framework », Françoise Lionnet, «Evading the Subject: Narration and the City in Ananda Devi's Rue la Poudrière », L'Esprit créateur, Postcolonial Representations: Women, Literature, Identity, Ithaca, Cornell University Press, 1995, p. 52.

27 «Ananda Devi's first published novel, Rue la Poudrière, is presented as a "feu brulant d'introspection", a passionate inner voyage, a mystical quest of a self- deprived of all freedom of choice, a self that in fact may never have existed », Danielle Chavy Cooper, «Rue la Poudrière », World Literature Today, 68, 1990, p. 515.

28 «All in all, Rue la Poudrière is a gripping, powerful, relentless novel, a mystical poetic epic. One of a kind », Ibid., p. 515.

${ }^{29}$ Vicram Ramharai, «La Ville de Port-Louis dans Rue la Poudrière d'Ananda Devi », L'océan Indien dans les littératures francophones, Paris, Karthala, 2001, p. 373.
} 
gens d'une certaine condition sociale $»^{30}$. De ce fait, Ramharai conclut que « le choix de Port-Louis comme cadre romanesque permet à Ananda Devi d'évoquer un espace qui fonctionne comme un piège $»^{31}$. En outre, Rohini Bannerjee remarque que dans Rue la Poudrière, Devi a mis l'accent sur les déséquilibres qui peuvent exister entre les philosophies hindoues de base et la vie urbaine contemporaine dans la société mauricienne ${ }^{32}$. Pour ce qui est du roman Le Voile de Draupadi, Cooper constate qu'il combine habilement des thèmes, personnages, événements et humeurs ${ }^{33}$. Elle note la clarté stylistique de ses expressions chaleureuses, de ses métaphores poétiques, et de son lyrisme qui ajoute à la charge émotionnelle ${ }^{34}$. Véronique Bragard, pour sa part, mentionne l'importance et le symbolique des éléments et affirme que Le Voile de Draupadi «est un ouvrage réaliste et psychologique » ${ }^{35}$. Pour Le Sari vert, Rohini Bannerjee observe que c'est «un roman sensoriel, passionnant et innovateur [qui] à travers un lyrisme électrique [...] plonge dans le créole, l'anglais et l'hindoustani, [...] explore la modernité de la violence $»^{36}$. Magali Marson, quant à elle, pense que Le Sari vert « est l'image d'une condition féminine détruite avant l'éclosion, ayant pour seuls recours le hurlement du silence ou la déviance par rapport aux normes érigées » ${ }^{37}$. Elle remarque également que le « réquisitoire contre la violence » ${ }^{38}$, exprime une «barbarie inexcusable, dont seul l'humain est capable $»^{39}$. Pour Christine Rousseau, le roman est « un huis clos à haute tension [où] Ananda Devi se tient sur le fil de l'(in)humanité, comme pour mieux révéler les ombres sulfureuses qui nous habitent $»^{40}$. Enfin, Yves Chemla juge que «le roman d'Ananda Devi luit de cette beauté qu'ont les visages réduits au silence par la folie masculine, mais dont les espaces intérieurs sont balayés

\footnotetext{
${ }^{30}$ Ibid., p. 383.

${ }^{31}$ Ibid.

${ }^{32}$ Rohini Bannerjee, «Daughter Forsaken: la résistance of the Indo-Mauritian Girl Child in Ananda Devi's Novel» dans India in the World, Cristina M. Gámez-Fernández, Antonia Navarro-Tejero, Cambridge Scholars Press, 2011, p. 125.

33 «Multifaceted and intense in subject matter, Le Voile de Draupadi skillfully interweaves themes, characters, events and moods », Danielle Chavy Cooper, «Le Voile de Draupadi», World Literature Today, 68, 1994, p. 640.

34 «Her lyricism flows easily and adds to the emotional charge », Ibid., p. 515.

${ }^{35}$ Véronique Bragard, «Cris des femmes maudites, brûlures du silence, la symbolique des éléments fondamentaux dans l'œuvre d'Ananda Devi », Notre librairie, 142, 2000, p. 73.

${ }^{36}$ Rohini Bannerjee, «Le Sari vert », Journal du conseil international d'études francophones (CIEF), 25, 1, 2010, p. 215.

${ }^{37}$ Magali Marson, «Ananda Devi. Le Sari vert», Cultures Sud. La Revue en ligne des littératures du sud, http://www.culturessud.com/contenu.php?id=42, consulté le 2 mai 2012.

${ }^{38}$ Ibid.

${ }^{39}$ Ibid.

${ }^{40}$ Christine Rousseau, «Le Sari vert d'Ananda Devi : un terrible monologue », http://www.lemonde.fr/livres/article/2009/11/12/le-sari-vert-d-ananda-devi_1266068 3260.html, consulté le 12 avril 2013.
} 
d'émotions que les mots, même insuffisants, parviennent quand même à repeupler ${ }^{41}$. Il conclut qu' «Ananda Devi répond à une interrogation radicale sur la condition féminine et le façonnement de celle-ci par les pseudo morales, et les échardes de l'histoire, qui façonnent l'île Maurice ${ }^{42}$.

Ainsi, les analyses faites par les chercheurs se concentrent principalement sur le style imagé, lyrique et poignant et sur les thèmes tels la quête mystique des personnages, le symbolisme des éléments ou la condition féminine. Ces chercheurs mentionnent également le milieu social, la tension psychologique des personnages femmes réduites au silence par les hommes. Pour cette étude, nous proposons de laisser de côté ces aspects déjà vus et de changer l'axe de la recherche en mettant l'accent sur le côté géographique, historique et sociologique des trois romans pour déterminer si ces romans dépeignent avec vérité la société mauricienne.

\section{Méthodologie}

Nous analyserons les trois romans choisis selon leur date de parution : Rue la Poudrière (1988), Le Voile de Draupadi (1993) et Le Sari vert (2009). Examiner les œuvres par ordre chronologique nous aidera en effet à mieux suivre d'éventuels changements au sein des trois romans, d'autant plus que Rue la Poudrière et Le Voile de Draupadi présentent très visiblement plus de références toponymiques que Le Sari vert. Qui plus est, puisque ces trois romans couvrent plusieurs décennies, les étudier par date de parution permettra de mieux suivre les événements historiques allant de la période pré-indépendante à la période post-indépendante. Enfin, cette approche nous aidera aussi à cerner s'il y a effectivement une évolution dans la société que présente l'auteure et si la place subalterne de la femme au sein du couple est perçue comme immuable.

Par ailleurs, pour étudier la dimension spatiale, nous examinerons la façon dont la toponymie, la flore et la faune sont décrites pour voir si le contexte local est privilégié par rapport à l'universel et pour mesurer le réalisme de l'univers décrit. L'étude de la dimension temporelle consistera à repérer les références historiques mentionnées dans les textes pour ensuite les comparer aux faits réels afin de juger de leur réalisme historique. Dans le chapitre quatre, nous nous intéresserons à la

\footnotetext{
${ }^{41}$ Yves Chemla, «Ananda Devi : Le Sari vert », http://www.cec-ong.org/index.php?option=com content\&view=article\&id=416:ananda-devi-le-sari-vert205-\&catid=131:chroniques-de-livres\&Itemid=224, consulté le 11 avril 2013.

${ }^{42}$ Ibid.
} 
dimension sociale, en étudiant la classe associée aux personnages, leur communauté et leurs conditions de vie dans le but d'évaluer le réalisme social des trois romans. Finalement dans la conclusion de la thèse, nous reviendrons sur les questions du local et du réalisme, de l'universalisation et du réalisme, du factuel et du fictionnel.

Comme nous l'avons vu, la plupart des chercheurs s'intéressant aux œuvres de fiction de Devi sont d'avis, et pour certains, ceci semble même indiscutable, que celleci dresse le portrait de l'île Maurice tout en mettant en avant les failles de la société mauricienne ${ }^{43}$. Ainsi, les analyses se portent sur les maux sociaux mauriciens, et démontrent comment Devi apporte, la plupart du temps, une lueur d'espoir en proposant aux femmes de prendre leur vie en $\operatorname{main}^{44}$. Or, nous remarquons que, Devi a plusieurs fois précisé dans ses entretiens que même si ses romans sont inspirés par la situation de l'île Maurice, les thèmes qui y figurent ne se limitent pas à l'île :

J'écris de la fiction et il se trouve que le lieu qui m'enracine le plus, c'est Maurice. Mais la portée de mes livres dépasse, je l'espère, la seule société mauricienne, et mes romans parlent d'individus qui débouchent sur des archétypes universels. Je dirais plutôt que ces personnages peuvent exister n'importe où et que l'île où se situent mes livres est plutôt une île fictionnelle que réelle ${ }^{45}$

\footnotetext{
${ }^{43}$ «Son écriture poétique contraste avec la violence de ses romans, la rage de ses héroïnes : des Mauriciennes broyées par une société machiste mais qui trouvent la force de s'émanciper malgré tout », Julien Burri, « Ananda Devi : la rage et le sucre », http://www.hebdo.ch/ananda_devi_la_rage_et_le_sucre_135983_html, consulté le 07 août 2013 ; « D’origine indo-mauricienne mais témoin d'exil et mélanges culturels complexes, Devi écrit sur tous les aspects de la société mauricienne sans "ethniciser" sa créativité », Véronique Bragard, Srilata Ravi, Ecritures mauriciennes au féminin: penser l'altérité, Paris, L'Harmattan, 2011, p. 12; «Rue la Poudrière and Moi, l'Interdite are two of Devi's works which are the most sensitive to the identity question facing the modern Mauritian generation, in particular, to its sentimental attachment to certain parts of its culture and historical background. At the same time, this generation, including the girl children of Paule and Mouna, are attempting to not only construct an identity that is authentically Mauritian but one that pays homage to their country of origin [...] Paule and Mouna not only face the challenges of being girl children born to a Hindu family, they are Hindu children living in Mauritius », Rohini Bannerjee, «Daughter Forsaken : la résistance of the Indo-Mauritian Girl Child in Ananda Devi's Novels » dans India in the World, Cristina M. Gámez-Fernández, Antonia Navarro-Tejero, Newcastle, Cambridge Scholars Press, 2011, p. 130.

${ }^{44}$ «In both Rue la Poudrière and Le Voile de Draupadi, Devi demonstrates that negotiating identity is not just a difficult process, but more often than not an impossible task [...] If the question of identity brings about such suffering as has been analysed in this article then, Devi suggests we should not persist in our quest but should simply be », Ashwiny Kistnareddy, "Interrogating Identity: Psychological Dislocations in Ananda Devi's Novels », Dalhousie French Studies, 94, Spring 2011, p. 36-37.

${ }_{45}$ Nathalie Philippe, «Explorer l'île pour atteindre l'universel. Retour sur l'œuvre d'Ananda Devi », Les Dépêches de Brazzaville, http://www.brazzaville-adiac.com/medias/dossiertele/suplit7.pdf, consulté le 13
} 
De ce fait, nous notons, à l'instar de Françoise Lionnet, Ritu Tyagi, Eileen Lohka et Odile Cazenave ${ }^{46}$, qu'il existe une divergence entre ce que la plupart des chercheurs ont trouvé et ce que dit Devi. Ainsi, dans notre recherche, nous tenterons d'analyser de près l'univers des personnages afin de voir si les romans de Devi dépeignent exclusivement la société mauricienne, ou s'ils s'ouvrent vers des horizons divers pour souligner des vérités plus universelles.

\section{Le local et l'universel}

Puisqu'un des grands axes de notre recherche est de dégager le local de l'universel, nous nous interrogerons sur le caractère de l'univers des personnages et sur leurs conditions de vie. Pour ce travail, nous retenons que le local est le fait de rester cloisonné et restreint dans un espace particulier du point de vue toponymique, donc, dans ce cas, dans celui de l'île Maurice. C'est aussi le fait de rester dans un cadre spécifique et particulier. Ainsi, dans ce contexte, le local s'exprimera par rapport à la géographie mauricienne, au passé historique mauricien et la société mauricienne dans son ensemble. L'universel au contraire, sera ce qui est hors de ce cadre local, ce qui part vers un autre lieu, en prenant une perspective plus large et au-delà du cadre spécifique de Maurice. Tout comme l'étude du local consistera à analyser la géographie, l'histoire et la société mauricienne, l'étude de l'universel consistera aussi, à examiner ces trois aspects, dans une perspective plus large. A ce propos, Ernest Dunn ${ }^{47}$, se demandant si la littérature africaine est confinée au local ou si elle part vers un universalisme, est d'avis que « les deux ne sont pas mutuellement exclusifs, mais sont

mars 2013; Mar Garcia, «Entretien avec Ananda Devi », La Tortue verte : revue en ligne des littératures francophones, http://www.latortueverte.com/Entretien\%20avec\%20Ananda\%20Devi.pdf, consulté le 5 septembre 2011, p. 3.

46 «Is this evasive self-positioning of the subject of the enunciation meant to evoke a universal female protagonist? », Françoise Lionnet, «Evading the Subject: Narration and the City in Ananda Devi's Rue la Poudrière », L'Esprit créateur. Post-colonial Women's Writing, 33, 1993, p. 17 ; " Devi's 'Mauritianness', therefore, lies in her openness to the universal, not in the insularity that the island can impose on Mauritians. In short, she chooses to embrace the expanse of the sea rather than the confinement of the land», Ritu Tyagi, "Rethinking Identity and Belonging. "Mauritianness" in the Work of Ananda Devi », dans Islanded Identities. Constructions of Postcolonial Cultural Insularity, New York, Rodopi, 2011, p. 106 ; « Ce lieu fluide [...], multiple et polymorphe, perçu autant de façon abstraite que concrète, se défait de la notion de frontière et englobe de ce fait une vision plus large de ce que l'écrivain tente de mettre en mots », Eileen Lohka, «Outrepasser le lieu et ouvrir un espace de création. Le cas d'Ananda Devi », Nouvelles études francophones, 28, 2013, p. 28-29 ; « L'œuvre d'Ananda Devi [en se référant au roman Les Hommes qui me parlent], ouverte sur le monde, l'ailleurs, l'autre, est traversée par un ensemble de motifs récurrents, notamment la présence d'une violence sourde », Odile Cazenave, «Les Hommes qui me parlent d'Ananda Devi. Un nouvel espace pour se dire ? », Nouvelles études francophones, 28, 2013, p. 39.

${ }^{47}$ Ernest Dunn, «Critical Perspectives: Local, Regional, Universal? », African Literature Today, 4, 1978, p. 52-55. 
plutôt liés ${ }^{48}$, et que le flux doit procéder à juste titre du particulier à l'universel ${ }^{49}$. Nous espérons pouvoir déterminer ce qu'il en est des trois romans d'Ananda Devi à partir des références textuelles. Si les traits repérés présentent des vérités qui dépassent le cadre mauricien, nous pourrons montrer que les romans sont portés vers l'universalisme, où l'universel supplante le local en dépassant les spécificités d'une culture ou d'une société, comme le suggèrent Devi, Lionnet, Tyagi, Lohka et Cazenave.

\section{Fiction et réalisme}

Un des grands axes de notre recherche étant la représentation de la réalité dans les trois romans, nous proposons de faire appel à des théoriciens qui se sont intéressés à la problématique du factuel dans le récit fictif. Nous aurons en particulier recours aux idées avancées par Guy de Maupassant, Roland Barthes, Mark Sainsbury, Névine El Nossery, Michael Riffaterre et Maureen Ramsden, qui se sont tous concentré sur l'idée de l'effet de réel dans le texte fictif.

Barthes est d'avis que le réalisme dans un récit fictif découle de l'importance des détails donnés même si ces derniers sont «superflus » ${ }^{50}$ et consistent d'un « remplissage $»^{51}$. A en croire Barthes, ce « remplissage » permet au lecteur de mieux situer le cadre et de comprendre l'ambiance et l'atmosphère qui règnent dans le roman. Si pour un lecteur mauricien, évoquer un cadre tropical n'est pas difficile, le lecteur "étranger" doit imaginer l'environnement à partir de la description détaillée et réaliste, par exemple, des frangipaniers, jacarandas, papayes rouges, cannes à sucre de l'île. Mark Sainsbury pense également qu'un texte fictif peut contenir des références directes à la réalité que le lecteur doit accepter comme telles si l'auteur a intégré des éléments relevant de la couleur locale dans son texte ${ }^{52}$. Nevine El Nossery précise que la fiction a pour rôle de « former, façonner ou modeler l'expérience pour la représenter et non à proprement parler l'inventer $»^{53}$, et de ce fait, « la fiction a [...] le pouvoir de susciter la réalité tout en maintenant une distanciation critique $»^{54}$. Maureen Ramsden, pour sa

\footnotetext{
48 « The two are not mutually exclusive, but rather, often go together », Ibid., p. 53.

49 « The flow should rightfully proceed from the particular to the universal », Ibid., p. 53-54.

${ }^{50}$ Roland Barthes, « L’Effet de réel », Communications, 11, 1968, p. 84.

${ }^{51}$ Ibid.

52 «Many fictions contain straightforward truths, which the reader is supposed to take as true. In such cases, the author provides "local color" by telling us things which not only are true, but which the reader is intended to realize he is supposed to treat as true », Mark Sainsbury, Fiction and Fictionalism. New Problems of Philosophy, London, Routledge, 2010, p. 4.

${ }^{53}$ Névine El Nossery, Témoignages fictionnels au féminin. Une réécriture des Blancs de la guerre civile algérienne, New York, Rodopi, 2012, p. 29.

${ }^{54}$ Ibid., p. 222.
} 
part, examine les barrières qui peuvent exister entre fiction et factuel ${ }^{55}$ et pense que chacun perçoit le monde en fonction de son propre contexte social et religieux. Pour elle, il existe de la fiction dans tout texte factuel et des éléments factuels dans tout texte fictionnel. Ainsi, il est difficile pour elle de tirer une ligne de démarcation claire entre fait et fiction. Michael Riffaterre maintient que le roman, tout en étant fictif, doit être vraisemblable pour retenir l'attention des lecteurs ${ }^{56}$. Il explique, de son côté, que le vraisemblable résulte d'une représentation verbale de la réalité, sans être la vérité ellemême ; c'est ce qu'il appelle la fictionnalisation ${ }^{57}$ de la réalité. Nevine El Nossery renchérit que le vraisemblable est «la substitution de l'expérience réelle ou hypothétique par une structure rhétorique imagée, qui néanmoins semble véridique et que le lecteur tend à accepter ${ }^{58}$. D'ailleurs, Guy de Maupassant avait déjà observé que le romancier projette une image de la réalité qu'il veut faire parvenir aux lecteurs. Maupassant avance aussi que chaque personne a sa propre perception de la réalité qui n'est qu'une «illusion du monde ${ }^{59}$. Cette représentation réaliste permet de présenter, selon lui, une «illusion complète du vrai $»^{60}$, « une image exacte de la vie ${ }^{61}$. Nous pourrions ainsi résumer ces notions des divers théoriciens par ce qu'avance Barthes, que lorsqu'un texte est vraisemblable, il se crée un effet de réel qui fait croire que tout ce qui a été écrit relève de la vérité.

Pour notre étude, nous retenons de ces propos que le réalisme, dans un texte fictif, est basé sur la représentation vraisemblable de la réalité, c'est-à-dire d'une vérité géographique, historique ou sociale. Dans un roman, le réalisme découle donc de l'expression du factuel et du vraisemblable qui produit un effet de réel, ou illusion de réel. En somme, aucun texte fictif n'est complètement fictionnel puisqu'il relate, d'une façon ou d'une autre, des éléments réels que le lecteur connaît plus ou moins bien. Pour faire croire à la réalité, le romancier a recours au vraisemblable et à des détails basés sur la réalité. Ces considérations théoriques forment le fondement de notre analyse des allusions spatiales à l'île Maurice, des faits historiques comme la traversée des

\footnotetext{
${ }^{55}$ Maureen Ramsden, « Fictional Frontiers: the Interrelation of Fact and Fiction between the World and the Text », Neophilologus, 95, 2011, p. 341-358.

56 «Its very name declares its artificiality and yet it must somehow be true to hold the interest of its readers », Michael Riffaterre, Fictional Truth, London, The John Hopkins University Press, 1990, xii.

${ }^{57}$ " Verisimilitude is an artifact, since it is a verbal representation of reality rather than reality itself : verisimilitude itself, therefore, entails fictionality », Ibid.

${ }^{58}$ Névine El Nossery, Témoignages fictionnels au féminin. Une réécriture des Blancs de la guerre civile algérienne, New York, Rodopi, 2012, p. 69.

${ }^{59}$ Guy de Maupassant, « Le Roman », dans Pierre et Jean, Paris, Albin Michel, 1970, p. 836.

${ }^{60}$ Ibid., p. 835 .

${ }^{61}$ Ibid., p. 833.
} 
travailleurs engagés indiens, et les émeutes dans les établissements sucriers, et des conflits raciaux et faits sociaux qu'Ananda Devi intègre, tels la prostitution, la pauvreté, le viol conjugal, l'homicide et la violence domestique.

\section{La microsociologie}

Nous nous appuierons sur la microsociologie pour étudier l'univers social des personnages ainsi que leurs relations au sein de leur classe et de leur famille.

Par microsociologie, nous comprenons l'étude d'un petit groupe social, à l'opposé de la société dans son ensemble. Stephen Sanderson, distinguant la microsociologie de la macrosociologie, précise que la microsociologie étudie l'ensemble des habitudes intellectuelles et comportementales au sein d'un petit groupe social tandis que la macrosociologie examine à grande échelle les façons de vivre et s'intéresse donc à l'économie, à la politique, au mode de vie des familles aussi bien qu'au fonctionnement des systèmes religieux ${ }^{62}$. Nous nous placerons dans la perspective de la microsociologie puisque nous cherchons à observer les relations des personnages masculins et féminins au sein du couple et de leur groupe social et nous nous concentrerons sur l'observation de leurs attitudes et comportements, de leur fonctionnement et mode de vie dans les trois romans.

Par ailleurs, le sociologue Joel Charon explique que toute société est divisée en classes sociales qui sont souvent déterminées par la situation économique de la personne. Ainsi, être riche signifie avoir plus de pouvoir et être en mesure de plus influencer l'ordre social et politique qu'une personne de classe moyenne ou inférieure. Il estime également que la société fait partie intégrante de la vie de l'être humain à travers sa socialisation ${ }^{63}$, c'est-à-dire, à travers les rapports qu'il développe avec les autres au sein de son groupe social. De ce fait, notre travail se concentrera sur l'étude de la relation, cordiale ou hostile, qu'entretiennent les différents membres des communautés dépeintes dans les trois romans. Précisément, nous analyserons la représentation des communautés mauriciennes, tant hindoue et musulmane que créole, et des différentes classes décrites dans les trois romans. Ceci nous amènera à nous intéresser aux registres de langue qui peuvent être indicatifs d'une classe sociale particulière. Ensuite, à un deuxième niveau, nous rapporterons en détails les rapports qu'entretiennent les personnages sur le plan interpersonnel au sein du groupe plus

\footnotetext{
${ }^{62}$ Stephen Sanderson, Macrosociology. An Introduction to Human Societies, New York, Harper \& Row Publishers, 1988, p. 2.

${ }^{63}$ Joel Charon, The Meaning of Sociology, Englewood Cliffs, Prentice-Hall, 1987, p. 51.
} 
restreint, notamment la relation entre mari et femme, et entre parent et enfant. Notre recherche couvrira donc ces deux aspects de la microsociologie; les relations intercommunautaires ou inter-classes et les relations intrafamiliales.

De surcroît, nous nous intéresserons aux travaux d'Emile Durkheim et de Robert Merton. Durkheim, affirme à propos des classes sociales que la division en classes d'une société est nécessaire mais ne doit pas être une barrière entre les hommes car elle doit promouvoir et améliorer l'autonomie de l'être humain même si ce dernier est convaincu qu'être autonome signifie avoir des liens de solidarité avec les autres ${ }^{64}$. Ainsi précise-t-il que cette solidarité est nécessaire pour consolider la stabilité et les liens sociaux et assurer que l'individualisme ne mène pas à la décadence ${ }^{65}$. Cette question de division de classe sociale sera particulièrement utile pour analyser l'univers fictif des personnages, en comparaison de la société mauricienne.

De son côté, Emile Durkheim s'est plus particulièrement penché sur la question d'anomie qu'il définit comme le dérèglement social qui se produit lorsqu'une personne n'est pas en mesure de répondre à ses attentes personnelles et à son statut social ${ }^{66}$. Pour Marco Orrù, l'anomie se présente plutôt lorsqu'il y a une irrégularité ou une absence d'homogénéité sociale dans toute la société ${ }^{67}$. Selon lui, puisque la société est le moteur des règles morales, l'anomie consiste en un relâchement des règles morales et judiciaires ${ }^{68}$. C'est dire que l'anomie fait référence aux restrictions sociales qui peuvent limiter les aspirations de l'individu, ou encore, au conflit qui émerge lorsque les croyances personnelles d'un individu s'opposent au système préétabli d'une société ${ }^{69}$. Cette notion d'anomie, fort intéressante, sera utile pour comprendre comment, dans les romans à l'étude, certains personnages tentent sans succès de s'intégrer dans la société en suivant des normes établies et finissent par aller à l'encontre de ces règles.

\footnotetext{
64 «In this respect resembling Max Weber, Durkheim wanted to enhance the autonomy of the individual even as he clung to the idea that such autonomy could only be attained upon secure foundations in conditions of social solidarity firmly binding its members together », Emile Durkheim, The Division of Labour in Society, London, Macmillan Publishers, 1984, p. xiii.

65 «Durkheim was convinced that without stable social bonds, without social solidarity, individualism would lead to the decay of society », Ibid., p. xiv.

${ }^{66}$ Emile Durkheim, Le Suicide. Etude de sociologie. Livre premier: les facteurs extra-sociaux, http://classiques.uqac.ca/classiques/Durkheim_emile/suicide/suicide_Livre_1.pdf, consulté le 16 décembre 2013, p. 7.

67 « Anomie becomes any form of deregulation or lack of cohesion from which society may suffer », Marco Orrù, Anomie, London, Allen \& Unwin, 1987, p. 107.

68 «Society is the mainspring of moral rules; anomie is the negation of society and hence the negation of all morality; any loosening of societal rules, whether moral or judicial, can be labeled as anomie », Ibid., p. 107-108.

69 «For some writers, anomie is the absence of cultural restraints on human aspirations: for others, it denotes a conflict of belief-systems in a society », Ibid., p. 1.
} 
Par ailleurs, Durkheim, s'intéressant aux causes du suicide, pense que celui-ci est une conséquence directe d'un malaise social causé par des inégalités. Selon lui, l'inégalité dans la distribution des biens peut amener une personne à recourir à des comportements déviants ou asociaux parce qu'il est dépossédée de ses valeurs sociales ou de sa raison d'être. Or, nous avons remarqué dans les trois romans que certains personnages n'hésitent pas à pratiquer la prostitution ou la magie noire pour gagner plus d'argent. Nous constatons aussi que certains personnages sont en conflit permanent avec les autres membres de leur famille à cause de valeurs sociales qui les étouffent. Cette perspective permettra d'expliquer la déchirure familiale dans Rue la Poudrière et Le Voile de Draupadi aussi bien que la mort étrange de la femme du médecin dans Le Sari vert qui se laisse mourir sous les yeux de sa fille et de son mari après avoir été ébouillantée avec du riz.

Pour sa part, Robert Merton analyse les phénomènes qui peuvent émerger chez une personne ou dans une société dominée par une autre, tels que le ressentiment, la déviance, la rébellion, et la dysfonction. Selon lui, le ressentiment se manifeste quand une personne éprouve de la haine, de l'envie ou de l'hostilité parce qu'elle n'a pas la capacité d'exprimer ses sentiments violents envers son agresseur ${ }^{70}$. Il y a déviance quand la personne rejette les pratiques institutionnelles sans que les aspirations de cette culture soient remises en cause ${ }^{71}$ et dysfonctionnement social quand il y a violation d'un code éthique ${ }^{72}$. La rébellion émerge quand les valeurs de la société et les difficultés de les suivre génèrent des comportements déviants qui vont à l'encontre du bon fonctionnement de la société ${ }^{73}$. Merton remarque que la rébellion, qui touche surtout ceux qui sont au bas de l'échelle sociale, contribue à la formation de sousgroupes unis mais en marge de la société ${ }^{74}$. Les "dysfonctions" découlent de comportements qui ne sont pas conformes aux normes ${ }^{75}$ de la société. En fait, ce qui

\footnotetext{
${ }^{70}$ «Diffuse feeling of hate, envy and hostility [...] a sense of being powerless to express these feelings actively against the person [...] evoking them », Robert Merton, Social Theory \& Social Structure, New York, The Free Press, 1968, p. 209.

71 « This [...] refers to the rejection of institutional practices but the retention of cultural goals », Ibid., p. 230.

${ }_{72}$ «Assumptions that deviant behaviour is necessarily equivalent to social dysfunction, and social dysfnction, in turn, to violation of an ethical code », Ibid., p. 237.

${ }^{73}$ «It is the conflict between culturally accepted values and the socially structured difficulties in living up to these values which exerts pressure toward deviant behavior and disruptive of the normative system », Ibid., p. 245.

${ }^{74}$ «Rebellion is confined to relatively small and relatively powerless elements in a community, it provides a potential for the formation of subgroups, alienated from the rest of the community but unified within themselves », Ibid.

75 «Dysfunctions [are] those observed consequences which lessen the adaptation or adjustment of the system », Ibid.
} 
paraît fonctionnel pour certains, peut être dysfonctionnel pour d'autres, et vice versa. Ces concepts aideront à appréhender le comportement malveillant ou destructif de certains personnages et à expliquer les causes de séparation de certains couples dans les trois romans.

L'ensemble de toutes ces notions de microsociologie nous permettra d'étudier et d'analyser l'univers social des personnages et leurs aspirations ainsi que d'établir si les individus et les couples suivent les normes de leur groupe ou s'ils se rebellent contre les règles d'une société qui les empêchent de se réaliser sur le plan personnel. De plus, nous serons dans la possibilité d'appréhender les situations précaires des personnages, et surtout d'expliquer le malaise social qui règne non seulement à l'île Maurice, mais ailleurs dans le monde. Ainsi faisant, nous pourrons conclure comment l'anomie bascule les fondements d'une famille et mène à un dysfonctionnement social.

\section{La coolitude}

Dans le but de mieux comprendre le fonctionnement et les réactions des personnages hindous dans les trois romans, il nous sera indispensable de nous référer au concept de coolitude qui a été élaboré par le poète mauricien, Khal Torabully. Inspiré par le terme de négritude qui fait allusion à une situation initiale similaire ${ }^{76}$, il perçoit la coolitude comme faisant référence à l'expérience des Indiens émigrés qui sont partis travailler loin de leur village natal, ainsi qu'à la prise de conscience de ces derniers qui ont fini par accepter leur exil et par s'adapter à leur terre d'accueil ${ }^{77}$. En fait, Khal Torabully considère la notion de coolitude surtout en référence à la traversée de l'océan Indien entreprise par les coolies indiens et au processus d'adaptation et d'acceptation de leur terre d'exil. D'après Torabully, la coolitude est « une remise en perspective ${ }^{78} \mathrm{du}$ passé culturel des coolies qui a été «mis à mal » ${ }^{79}$ et de leur ajustement à une terre d'exil, à un environnement hostile et à des cultures préexistantes, notamment africaine et européenne. De ce fait, Torabully affirme que même si la coolitude a une relation avec l'Inde, elle n'a pas pour autant son noyau dans ce pays, car ce dernier n'est que le point de départ et non l'ultime but ${ }^{80}$. Pour Jean-Georges Prosper, poète mauricien de

\footnotetext{
76 « Négritude and Coolitude sprang from the same tragedy », Marina Carter, Khal Torabully, Coolitude. An Anthology of the Indian Labour Diaspora, London, Anthem Press, 2002, p. 147.

77 «Coolitude confronts the experience of Indians beyond the seas, and [...] the elaboration of the awareness of the Indian who has accepted his exile, and acquired new forms of expression, to become part of the history of the nations in which he has settled », Ibid., p. 214.

${ }_{78}^{7 b i d ., ~ p . ~} 144$.

${ }^{79}$ Ibid., p. 145.

${ }^{80}$ Ibid., p. 147.
} 
renom, la coolitude est une « sorte de négritude à l'indienne » ${ }^{81}$, faisant écho à Senghor qui avait défini la négritude comme étant une idéologie qui restaure les valeurs des Noirs qui tentent de retrouver leur dignité dans un monde colonial qui les avait privés d'humanité $^{82}$. Pour lui, la coolitude souligne le fait que des milliers d'Indiens se sont exilés de leur pays pour trouver du travail comme travailleur engagé en s'exilant. Pour d'autres, ce concept renvoie spécifiquement à la notion de mélange des cultures. Il conduit donc à un imaginaire et à une prise de conscience transculturelle ${ }^{83}$. Ainsi, Véronique Bragard affirme que la coolitude fait référence à la traversée cauchemardesque du coolie, à la fois en tant que migration historique et comme métonymie de la rencontre des cultures. En mettant l'accent sur le voyage, le lien avec l'Inde comme terre natale idéalisée est évacuée ${ }^{84}$.

Si le terme de coolitude est appliqué à toutes les colonies anglaises où les travailleurs indiens engagés étaient partis travailler, comme l'observe Torabully, il faut noter que c'est plustôt l'île Maurice que les Caraïbes qui a vu naître la notion de coolitude $^{85}$. Ce concept sera donc surtout appliqué pour l'étude du Voile de Draupadi puisque le grand-père d'Anjali fait partie de ceux qui ont traversé l'océan pour s'installer dans leur nouvelle terre d'acceuil. Une fois sur place, il a dû, comme les autres travailleurs engagés, faire face aux conditions terribles de travail dans les champs de canne pour assurer une meilleure vie à sa famille et les sortir de la misère. Cette conception sociologique relative aux Hindous de l'île Maurice servira également à l'analyse de la famille hindoue descendue de travailleurs engagés décrite dans $L e$ Voile de Draupadi et Le Sari vert.

\section{Le rêve mauricien}

Pour pouvoir conclure les analyses menées dans le chapitre sur la dimension sociale, nous concevons le terme de 'rêve mauricien' qui fait allusion à l'ambition de

\footnotetext{
${ }^{81}$ Ibid., p. 15.

82 « Négritude [...] refers to the values of the Black man, and represented an attempt to give him his dignity in a colonial world in which Negro seemed to be almost devoid of humanity », Ibid., p. 146.

83 « Coolitude is an aesthetic blend, a kind of mix of a complex culture, bringing to the imaginaire a part of the other. It calls to attention "Indianness" in relation with 'otherness' as a premise which leads to a transcultural awareness », Ibid., p. 168.

84 « [...] is not based on Coolie as such but relies on the nightmare transoceanic journey of Coolies, as both a historical migration and a metonymy of cultural encounters [...] By making the crossing central, coolitude avoids any essentialism and connection with an idealized Mother India, which is clearly left behind », Ibid., p. 15.

85 «It is Mauritius, rather than the Caribbean, which has provided the inspiration for this poetry of créolité and for the birth of coolitude », Ibid., p. 215- 216.
} 
tout Mauricien, quelles que soient sa communauté ou sa classe sociale, de monter l'échelle sociale afin de jouir d'une vie plus confortable.

Si cette notion semble découler de l'American dream, qui fait référence à des principes qui encouragent les gens en dehors de toute appartenance ethnique, à prospérer et à monter l'échelle sociale, la notion de 'rêve mauricien' ne se base pas sur les mêmes idéologies. En effet, même si le 'rêve mauricien' concerne la société mauricienne dans son ensemble, la notion n'est pas fondée sur les bases égalitaires de l'American dream, où le dur labeur est la clé à la réussite financière et sociale. Le concept de 'rêve mauricien' implique plutôt un certain individualisme qui peut mener à la corruption, à la débauche ou à la dépravation dans le simple but de progresser et d'accéder à la richesse matérielle. Si l'American dream signifiait que les gens travaillaient d'arrache-pied pour atteindre leur but grâce à leur détermination et à leur dur labeur, le 'rêve mauricien' consiste plutôt à avoir recours à des actions plus ou moins illégales pour atteindre son but. Le 'rêve mauricien' incite surtout le riche à devenir plus riche en lui permettant d'éliminer ceux qui vont à l'encontre de leur ambition personnelle, même si cela implique voler, corrompre, vendre ou tuer les siens. Sachant que les trois romans dépeignent, entre autres, des personnages corrompus et égocentriques qui vivent dans une société très inégale, ce concept permettra, par exemple, d'éclairer les comportements de Marie et de Mallacre dans Rue la Poudrière, de Dev et de Faisal dans Le Voile de Draupadi, et de Bissam Sobnath dans Le Sari vert.

Pour bien cerner ce concept de 'rêve mauricien', il est essentiel d'expliquer plus le fonctionnement de la société mauricienne contemporaine, complexe et unique comme le constate Jean-Georges Prosper: «les Mauriciens ne sont pas un peuple autochtone. Issus de souches européenne, africaine et asiatique depuis deux siècles seulement, les Mauriciens se distinguent par une personnalité hybride et complexe ${ }^{86}$. De fait, cette société multiculturelle, multiraciale et multilingue se compose officiellement des Indo-Mauriciens, formés de deux groupes différents, Hindous et Musulmans ${ }^{87}$, des Sino-Mauriciens, et de la population générale ${ }^{88}$, terme qui fait

\footnotetext{
${ }^{86}$ Jean-Georges Prosper, Histoire de la littérature mauricienne de langue française, Rose-Hill, Editions de l'océan Indien, 1978, p. 8.

87 « The differentiation on religious lines was acknowledged in the 1962 census which enumerated the Indo-Mauritian population in two ethnic communities: Hindus and Muslims », Amit Mishra, Mauritius, Delhi, National Book Trust, 2009, p. 134.

${ }^{88}$ « General population, when first conceived by census officials, was a bracket for all the people who could not be identified as Indo-Mauritians or Chinese [...] This category comprises of white people from French descent (Franco-Mauritian), coloured Creole population of mixed ancestry (African-European, African-Indian or African Chinese) and all people who does not appear, from his way of life, to belong to
} 
allusion aux descendants blancs des Français et des Créoles métisses d'origine africaine ${ }^{89}$.

Pour les Indo-Mauriciens, catégorie de la population qui est fréquemment représentée dans les romans de Devi, la priorité a toujours été d'acquérir une bonne éducation, d'avoir un travail respectable, de préférence dans le secteur gouvernemental, ou d'être propriétaires de terrains et d'acquérir un bien matériel. Dupon remarque que

par leur épargne, et grâce au morcellement de propriétés, les Indiens accèdent en grand nombre à la propriété constituant une classe de petits planteurs qui peu à peu arrive à détenir près de la moitié de la superficie des terres plantées en canne. C'est de cette classe dont sont les Indo-Mauriciens qui accéderont progressivement à beaucoup d'emplois possibles, y compris la fonction publique $^{90}$.

Si les Indo-Mauriciens travaillent, pour la plupart, dans le secteur gouvernemental ou exploitent les terres agricoles depuis longtemps, les Créoles sont, principalement, pêcheurs, travailleurs dans les ports, laboureurs, ou ouvriers dans les usines sucrières ou textiles ${ }^{91}$. Toutefois, de nos jours, « on trouve [...] des Créoles dans les postes les plus élevés du Civil Service ${ }^{92}$. Pour ce qui est des Sino-Mauriciens, plus de cinquante pour cent des entreprises commerciales, incluant de grands commerces, leur appartiennent ${ }^{93}$. L'aspiration à acquérir du bien matériel, à avoir un bon niveau d'éducation et à être à l'aise financièrement ne se limite donc plus aux Indo-Mauriciens mais touche en fait toutes les ethnies de cette société composite ${ }^{94}$ depuis que le programme «l'éducation pour tous », qui a débuté en 1950, a aidé la population à

one or the other of these three communities (Hindu, Muslim and Chinese) », Amit Mishra, Mauritius, Delhi, National Book Trust, 2009, p. 130.

${ }^{89}$ Pour de plus amples détails sur le regroupement de la population mauricienne : «2011 Housing and Population Census, Mauritius. Volume 2. Demographic and Fertility Characteristics », Ministry of Finance and Economic Development, http://www.gov.mu/portal/goc/cso/file/2011VolIIPC.pdf, consulté le 8 juin 2014, p. 68.

${ }^{90}$ J. F. Dupon, «La Société mauricienne », Revue juridique et politique, 23, 1969, p. 341.

${ }^{91}$ ThinkQuest, « Social Stratification in Mauritius », http://library.thinkquest.org/C0110237/Population/Social_Classification/social_classification.html, consulté le 13 janvier 2014.

${ }^{92}$ J. F. Dupon, «La Société mauricienne », Revue juridique et politique, 23, 1969, p. 353.

93 Amit Mishra, Mauritius, Delhi, National Book Trust, 2009, p. 132.

${ }^{94}$ J. F. Dupon, « La Société mauricienne », Revue juridique et politique, 23, 1969, p. 337. 
grimper l'échelle sociale ${ }^{95}$. De nos jours, tous les Mauriciens jouissent non seulement d'une éducation gratuite mais aussi d'un système de santé public gratuit. Les gens au bas de l'échelle ou à faible revenu reçoivent aussi des aides alimentaires, des pensions ou bénéficient même des logements sociaux de la NHDC (National Housing Development Company Ltd) qui leur permettent d'améliorer leurs conditions de vie.

Si le gouvernement s'efforce d'aider et d'unir la nation mauricienne, c'est aussi pour réduire la ségrégation, les injustices et les conflits ethniques. Pourtant, les différentes ethnies de l'île Maurice, qui sont obligées de cohabiter, restent bien démarquées : «on constate [...] qu'assez rares sont les habitants de ce pays qui se considèrent d'abord comme Mauriciens ${ }^{96}$. Ceci peut s'expliquer en partie par le fait qu'officiellement, les habitants sont catégorisés selon leur appartenance ethnique. Ceci contribue à ce que « le danger "communaliste" demeure très fort à l'île Maurice »". Connue comme la nation arc-en-ciel, l'île Maurice a vu dans le passé des incidents raciaux, notamment en janvier $1968^{98}$ et en février $1999^{99}$, qui indiquent qu'il reste malgré tout des inégalités sociales qui affectent surtout les Créoles encore stigmatisés par l'esclavage ${ }^{100}$, vu que ces deux conflits raciaux impliquaient les Créoles. De ce fait, le communautarisme est bel et bien toujours présent : «On pourrait presque dire qu'il fait partie du folklore local. On le trouve dans tous les secteurs de la vie ${ }^{101}$ et cela malgré le slogan nationaliste "enn sel lepep enn sel nation" (un seul peuple, une seule nation) ${ }^{102}$. Malgré ces divisions ethniques et sociales qui subsistent, l'île Maurice connaît un succès économique certain, souvent appelé «miracle mauricien » ${ }^{103}$, et le peuple mauricien continue son ascension vers la réussite matérielle. C'est là la preuve

\footnotetext{
95 Amit Mishra, Mauritius, Delhi, National Book Trust, 2009, p. 137.

${ }^{96}$ J. F. Dupon, « La Société mauricienne », Revue juridique et politique, 23, 1969, p. 342.

97 Ibid., p. 356.

98 Anonyme, «La vague de violence à Port-Louis. La Riot Unit et la SMF prêtes à intervenir », Week- End, $\mathrm{n}^{\mathrm{o}}$. 69, 21 janvier 1968, page inconnue.

${ }^{99}$ Catherine Boudet, «Emeutes et élections à Maurice. La mort de Kaya, aléa ou échec de la construction nationale ? », Politique africaine, 79, 2000, p. 153-164.

100 «Faced with this institutional void, creoles today find themselves in a situation of social exlusion in the Mauritian society. They feel indeed sometimes referred to as nation (lowly people) which is indicative of the fundamental discrimination against the poor black creoles, stigmatized by the past of their ex-slaves ancestors who were subsequently emancipated or considered as such in the colony », Suzanne Chazan-Gillig, "The Roots of Mauritian Multi-Culturalism and the Birth of a New Social Contract: Being “Autochtone”, Being Creole », Journal of Mauritian Studies, 2, 2003, p. 66.

101 Nooreeda Khodabocus, «ENN SEL LEPEP, ENN SEL NATION ?: Communalisme vs. Mauricianisme », http://www.lepep.net/2013/03/enn-sel-lepep-enn-sel-nation-communalisme-vs-mauricianisme/, consulté le 13 janvier 2013.

102 Véronique Bragard, «Matière à photographie: cosmopolitiques et modernité créoles à l'île Maurice », French Forum, 34, 2009, p. 76.

${ }^{103}$ Emmanuel Leroueil, «Le Miracle mauricien », http://terangaweb.com/le-miracle-mauricien/, consulté le 14 janvier 2014.
} 
qu'existe un 'rêve mauricien' qui fait redoubler d'effort les gens dans l'espoir d'une promotion sociale, et ceci sans distinction de race ou d'appartenance ethnique.

Les romans de Devi montrent ces clivages ethniques et sociaux et comment les personnages réussissent plus ou moins bien à échapper à leurs conditions d'origine. Ils font également référence aux conflits raciaux qui ont contribué à renforcer les divisions sociales et ethniques de la société mauricienne, et qui font que l'individu n'œuvre pas pour le bien-être de la société dans son entier, mais est de plus en plus individualiste et arriviste. Le 'rêve mauricien' fait que l'individu n'hésite pas à aller contre les normes sociales pour un gain financier personnel et à jouir de son pouvoir au sein de son entourage et de son groupe social. Nous nous demanderons comment les romans que nous examinons font état de ce phénomène social typique de Maurice, et nous en étudierons les manifestations au niveau des personnages. Nous nous intéresserons aussi aux couples afin de déterminer s'ils poursuivent ce rêve à l'unisson, ou si, au contraire, c'est ce phénomène social qui fait que les couples se brisent. 


\section{Partie II}

\section{Dimension spatiale}




\section{Introduction}

Dans cette partie, il sera question d'examiner dans les trois romans les aspects géographique et toponymique, la flore et la faune pour déterminer à quel point ces romans sont ancrés dans une réalité typiquement mauricienne. En ayant recours aux travaux de Michael Riffaterre, Vincent Jouve, ou encore Névine El Nossery, nous nous demanderons comme ces trois romans relèvent de la fiction et combien ils sont une représentation vraisemblable de la réalité géographique mauricienne.

Rue la Poudrière se déroule majoritairement à Port-Louis, la capitale de l'île Maurice, tout en faisant référence à certains endroits de la capitale, telles la Butte ou la rue la Poudrière alors que Le Voile de Draupadi, plus axé sur d'autres villes et villages de l'île, regorge d'indications sur la faune et la flore de toutes sortes. Le Sari vert est plutôt basé sur les grandes villes de Curepipe et de Port-Louis et la petite ville de Mahébourg. Contrairement aux deux autres romans, celui-ci ne dévoile guère de détails sur la flore et faune mauriciennes. L'analyse détaillée des descriptions de celles-ci vise à déterminer par quel biais l'espace littéraire de ces trois romans limite leur encadrement toponymique mais aussi comment ces espaces dépeints peuvent dépasser un territoire précis pour s'ouvrir sur l'universel.

\section{Rue la Poudrière}

Jusqu'à présent, très peu de chercheurs se sont préoccupés de l'aspect géographique, de la toponymie, de la faune et de la flore. Vicram Ramharai est un des rares critiques à avoir commenté cet aspect du roman. Il démontre comment la ville de Port-Louis peut être un espace néfaste pour les personnages : «espace inhumain ${ }^{104}$ ou «espace dégradé et dépravé » ${ }^{105}$. Dans son analyse, Ramharai explique que les Dockers' Flats, La Butte et rue la Poudrière sont «trois micro-espaces » ${ }^{106}$ qui font partie du «macro-espace ${ }^{107}$, qu'est Port-Louis. Il remarque que les Dockers' Flats «sont liés au port dans la mesure où ce sont les travailleurs du port qui y habitent ${ }^{108}$. Jean-Claude Abada Medjo, quant à lui, mettant l'accent sur les caractéristiques de la ville de Port-Louis, fait une comparaison entre Port-Louis et New Delhi dans Indian

\footnotetext{
${ }^{104}$ Vicram Ramharai, «La Ville de Port-Louis dans Rue la Poudrière d'Ananda Devi », L'océan Indien dans les littératures francophones, Paris, Karthala, 2001, p. 374.

${ }^{105}$ Ibid., p. 376.

${ }^{106}$ Ibid., p. 374.

${ }^{107}$ Ibid.

${ }^{108}$ Ibid.
} 
Tango. Il note que «comme à Port-Louis, île Maurice, les quartiers riches de New Delhi, en Inde, caractérisés par une architecture exceptionnellement géométrisée, sont réservés aux nantis, à des castes "supérieures", alors que les bidonvilles au profil architectural improbable sont le lot des défavorisés issus des castes "inférieures" » ${ }^{109}$. Dans un sens, Medjo rejoint Ramharai en comparant Port-Louis à «un ensemble spatio-temporel $»^{110}$ et les Dockers' Flats, la Butte et rue la Poudrière à «trois microchronotopes $»^{111}$. Si pour Ramharai, la ville garde les personnages «prisonniers ${ }^{112}$, pour Medjo, cet espace est un «piège ${ }^{113}$ pour Paule, qui est «comme toutes les filles rabotées par l'esclavage sexuel ${ }^{114}$. Par ailleurs, Nivoelisoa Galibert écrit que la ville de Port-Louis «n'a d'existence que par les référents propres à en permettre l'identification : une rue, La Poudrière ; un quartier, les dockers' flats » ${ }^{115}$ et affirme que « le roman élabore une sémantisation des dockers' flats conforme à l'univers mécanique et pessimiste des ports ${ }^{116}$. Pour sa part, Ritu Tyagi avance que les romans de Devi font le récit des jeunes Mauriciens marginaux ${ }^{117}$ et met l'accent sur la géographie et le cadre sombre dépeints dans Rue la Poudrière, où Port-Louis représente un piège et une impasse pour les personnages ${ }^{118}$, un lieu aliéné et inconnu ${ }^{119}$, une ville noire et hostile ${ }^{120}$.

Finalement, en gardant en tête ce qui a déjà été dit sur ce roman, nous procéderons à l'analyse de la toponymie, de la flore et de la faune dans Rue la Poudrière pour déterminer le degré de réalisme et de fiction, ce qui nous aidera à conclure à quel point cette œuvre est basée sur les aspects locaux de l'île Maurice.

\footnotetext{
109 Jean Claude Abada Medjo, « Poétique de la ville dans l'œuvre d'Ananda Devi », http://revel.unice.fr/loxias/index.html?id=7044, consulté le 17 janvier 2013, p. 6.

${ }^{110}$ Ibid., p. 4.

111 Ibid.

112 Vicram Ramharai, «La Ville de Port-Louis dans Rue la Poudrière d'Ananda Devi », L'océan Indien dans les littératures francophones, Paris, Karthala, 2001, p 374.

113 Jean Claude Abada Medjo, « Poétique de la ville dans l'œuvre d'Ananda Devi », http://revel.unice.fr/loxias/index.html?id=7044, consulté le 17 janvier 2013, p. 4.

115 Nivoelisoa Galibert, «Port de badge et vision panoptique : les Dockers' Flats de Port-Louis dans Rue la Poudrière, (1988) d'Ananda Devi, Revue Historique des Mascareignes, 5, 2004, p. 96.

116 Ibid., p. 100.

117 «In her novels, Devi recounts the tales of young Mauritians who belong to the marginalized strata of society and live out their lives against the background of the island's inherent cultural diversity », Ritu Tyagi, «Rethinking Identity and Belonging. "Mauritianness" in the Work of Ananda Devi », dans Islanded Identities. Constructions of Postcolonial Cultural Insularity, New York, Rodopi, 2011, p. 92.

118 «She presents an inhuman space limited not only by its geography but also by the poverty, suffering and misery that trap characters in an impasse », Ibid., p. 97.

119 « They are caught in an inevitable contradiction as the island home turns into an alienating insular space, and often becomes entirely unfamiliar », Ibid., p. 98.

${ }^{120}$ «Far from Hart's serene sculpted mountains, lit by a bright and luminous sun, Devi's Port-Louis is a dark and hostile world », Ibid., p. 99.
} 


\section{Géographie et toponymie dans Rue la Poudrière}

Rue la Poudrière a pour cadre la ville de Port-Louis mais les personnages sont ancrés dans des quartiers et faubourgs qui ne sont pas décrits de manière détaillée. Il est fait mention de «Marie, Reine de la Paix » ${ }^{121}$, l'illustre chapelle, sanctuaire et lieu de pèlerinage érigé en 1940 qui se trouve sur le flanc de «la montagne des Signaux » ${ }^{122}$, renommée à Port-Louis pour son phare qui guide les bateaux dans le quai de «la Grande Rivière Nord-Ouest ${ }^{123}$, réputée pour ses vingt-deux kilomètres de longueur, qui termine son cours à Port Louis, du «Champ de Mars » ${ }^{124}$, connu pour son hippodrome où «Edouard emmen(ait) Marie [...] pour les courses hippiques ${ }^{125}$, de «l'église de Cassis » ${ }^{126}$, célèbre église chrétienne, et de « la cathédrale de St. Louis » ${ }^{127}$, cathédrale bien connue des Mauriciens, complétée en 1933.

Outre ces brefs points de repère connus de Port-Louis, certains endroits sont décrits plus en détails. Ainsi, Paule est principalement associée à trois lieux spécifiques, selon les différentes périodes de sa vie. Son enfance se déroule aux Dockers' Flats, situés à Baie-du-Tombeau au nord de Port-Louis : "nous habitions alors un quartier réservé aux travailleurs des quais, les débardeurs et les stevedores, à l'époque où les salaires étaient encore minables $»^{128}$. Son adolescence se passe à la Butte, toujours à Port-Louis mais plus à l'ouest de la capitale. Devenue adulte, elle vit dans le centre de Port-Louis, dans la rue la Poudrière. En outre, la jeune fille perçoit Port-Louis comme « un personnage d'une étrange antiquité, une vieille créature décimée mais éternelle qui connaît le passé et toutes ses meurtrissures ${ }^{129}$. A d'autres occasions, Paule décrit la ville comme étant « antique [...] à la face de mendiante délurée » ${ }^{130}$, « fripé[e] comme une jupe trop lavée et trop séchée au soleil, avec ses tôles rouillées réfractant la chaleur, ses chemins rouges de poussière ${ }^{131}$, ou comme « [une] cité tentaculaire et vigoureuse, grand navire aux cales pouilleuses, aux ponts pleins de soleil $»^{132}$. Outre ces images lyriques, en décrivant à plusieurs reprises ces divers endroits de Port-Louis, Devi a fait

\footnotetext{
${ }^{121}$ Ananda Devi, Rue la Poudrière, Abidjan, Nouvelles Editions Africaines, 1988, p. 61.

${ }^{122}$ Ibid., p. 68.

123 Ibid., p. 134.

124 Ibid., p. 36.

125 Ibid.

${ }^{126}$ Ibid., p. 109.

127 Ibid.

${ }^{128}$ Ibid., p. 18.

${ }^{129}$ Ibid., p. 110.

${ }^{130}$ Ibid., p. 114

131 Ibid., p. 119.

${ }^{132}$ Ibid., p. 167.
} 
appel à ce que Michel Arrivé appelle les «toponymes quotidiens » ${ }^{133}$, qui sont, en fait, « des toponymes ordinaires : ceux qu'on utilise à tout moment de la vie quotidienne pour désigner un lieu, quel que soit ce lieu $»^{134}$.

En plus, les métaphores et comparaisons de Port-Louis accentuent son caractère générique de grande ville où la pauvreté, comme Barney Cohen l'explique, se manifeste clairement dans : des quartiers surpeuplés, un taux élevé de criminalité, des logements insalubres et un accès insuffisant à l'eau potable, à un assainissement adéquate et aux autres services ${ }^{135}$. De ce fait, nous constatons que la description faite de Port-Louis dans le roman est aussi celle de n'importe quelle ville en voie de développement. C'est pourquoi Danielle Cooper décrit le port comme étant une partie de la ville qui est identifiée à Port-Louis à l'île Maurice, mais [qui] pourrait aussi être peu importe quel port, peu importe quel bidonville notamment du tiers monde, $[\ldots]$ peu importe quel ghetto qui existe avec une population marginale qui lutte pour son existence, entouré d'un huis $\operatorname{clos}^{136}$.

Les Dockers' Flats sont dépeints comme « une termitière grouillante d'hommes, de femmes et d'enfants empaquetés dans les boîtes de savon de trois étages, six appartements aux pièces minuscules et sans air, "salle de séjour", chambre à coucher, salle de douche, cuisine, le tout contenu dans trente mètres carrés d'espace ${ }^{137}$. Or, pour Paule, l'appartement de sa famille constitue une spacieuse habitation de «luxe ${ }^{138}$. C'est aussi l'endroit où elle a établi « la seule amitié [qu'elle a] nourrie à cet âge de sables mouvants ${ }^{139}$ avec son amie Mireille qui lui a «appris la première l'intensité de ces voyages intérieurs à huis clos, qui avaient lieu entre ces quatre murs ${ }^{140}$. Cette description de Paule des Dockers' Flats est très vraisemblable puisque d'après les descriptions faites par Margaret, une ancienne habitante des Dockers' Flats,

\footnotetext{
${ }^{133}$ Michel Arrivé, « Toponymie et littérature », http://hal.inria.fr/docs/00/82/29/75/PDF/TOPONYMIE_LITTA_RATURE_PDF.pdf, p. 2, consulté le 1 mai 2014.

134 Ibid.

135 «The manifestations of urban poverty are clearly visible in all major cities: overcrowded neighborhoods, high rates of crime, inadequate housing and insufficient access to clean water, adequate sanitation and other services », Barney Cohen, « Small Cities, Big Problems », Science and Technology, 20, 2004, p. 87.

136 «It is identified as Port-Louis in the author's native Mauritius, but it might as well be any harbor, any shanty town in the Third World or beyond, anywhere a ghetto exists and a marginal population ekes out a miserable existence in a no-exit situation », Danielle Chary Cooper, «Rue la Poudrière », Notre librairie, 118, 1994, p. 171.

${ }^{137}$ Ananda Devi, Rue la Poudrière, Abidjan, Nouvelles Editions Africaines, 1988, p. 18.

138 Ibid.

${ }^{139}$ Ibid., p. 19.

${ }^{140}$ Ibid.
} 
cet endroit était « comme une île surpeuplée » ${ }^{141}$, où, « au climat qui régnait dans les Flats, il fallait ajouter l'état des lieux, où le crépi partait en lambeaux, mais là n'était pas le pire: lorsque les tanks de WC étaient bouchés, les appartements situés endessous empestaient, l'eau avec ses matières fécales s'infiltraient dans les chambres ${ }^{142}$. Ainsi, nous constatons que le témoignage de cette ancienne habitante des Dockers' Flats est très proche des descriptions données dans le roman. Dans ce cas, « la fiction a [...] le pouvoir de susciter la réalité » ${ }^{143}$ avec la description des bâtiments vétustes de Port-Louis. Et de ce fait, Devi «nous met en rapport avec des lieux intimes de la ville [et] construit une trame symbolique à travers laquelle nous lisons l'espace et les lieux urbains ${ }^{144}$ de ce roman. Et faisant le lien entre roman et lecteur, Vincent Jouve, en mentionnant les faits réels d'une œuvre, ajoute que « le texte ne peut décevoir l'attente du lecteur que dans une certaine limite. Ce dernier ne tolérera pas (surtout dans un genre aussi référentiel que le roman) de trop grandes distorsions entre le monde textuel et son monde de référence ${ }^{145}$.

Le roman est aussi ancré dans le port. C'est le lieu de travail du père de Paule, c'est aussi le quartier où Paule ressent « le plaisir de toutes les sensations réunies ${ }^{146}$. Il est question des bateaux immenses qui accostent au quai, des débardeurs qui effectuent l'embarquement et le débarquement des caisses. C'est par le regard de Paule, qui s'y rend souvent, que l'on suit son père, Edouard, et les autres ouvriers qui travaillent «avec la prestesse agressive des fourmis rouges » ${ }^{147}$. Une longue description du port insiste sur «les cargos [qui] glissent jusqu'aux quais [...] les remorques [qui] font le va-et-vient entre les bateaux de pêche [...] des chalutiers plus étroits [qui] accostent le quai [...] couleur de rouille ${ }^{148}$. Ce portrait du port de PortLouis peut en fait référer à n'importe quel port du monde où arrivent et partent les bâtiments de navigation pour l'embarquement et le débarquement du fret et des

\footnotetext{
${ }^{141}$ Pradeep Daby, «Des Dockers Flats aux nouvelles résidences : ce qui a changé pour des familles entières »,

http://www.defimedia.info/defi-plus/dp-societe/item/55709-des-dockers-flats-aux-nouvelles-residencesce-qui-a-change-pour-des-familles-entieres.html, consulté le 8 juin 2014.

142 Ibid.

${ }^{143}$ Névine El Nossery, Témoignages fictionnels au féminin. Une réécriture des Blancs de la guerre civile algérienne, New York, Rodopi, 2012, p. 222.

${ }_{144}^{145}$ Bertrand Lévy, « Géographie et littérature. Une synthèse historique », Le Globe, 146, 2006, p. 41.

${ }^{145}$ Vincent Jouve, L'Effet-Personnage dans le roman, Paris, Presses Universitaires de France, 1992, p. 95 .

${ }_{146}$ Ananda Devi, Rue la Poudrière, Abidjan, Nouvelles Editions Africaines, 1988, p. 22.

${ }^{147}$ Ibid.

${ }^{148}$ Ibid.
} 
passagers ${ }^{149}$. D'ailleurs, Paule ne remarque-t-elle pas : «qui ne connaît pas le port, son gris, sa lourdeur et son tumulte ne connaît pas le battement de Port-Louis ${ }^{150}$; suggérant bien la généralisation de ce lieu de transbordement. Ivan Seidl a noté que l'auteur peut ainsi faire appel à l'expérience du lecteur « à force de nommer des choses connues ou évidentes, l'auteur réussit à animer en nous un monde, non sans similitude avec celui où nous vivons $»^{151}$.

La Butte, située à l'ouest de Port-Louis, est un endroit plutôt commercial que résidentiel mais Rue la Poudrière n'offre que peu de détails sur cet endroit :

La Butte recevait, d'un côté, l'air de la montagne, de l'autre, l'effluve du terrain d'ordures qui servait de dépôtoir à toute cette partie de la cité. Ces deux courants trouvaient leur point d'impact dans l'agglomération de maisons où nous habitions, collées aux flancs comme des jumeaux siamois ${ }^{152}$.

Ainsi, en ne donnant pas de plus amples détails sur la Butte, nous concluons que ce sont les Dockers' Flats et rue la Poudrière qui ont marqué le plus Paule, sans doute parce qu'elle a grandi et mûri. Par ailleurs, ce peu de détails sur la Butte démontre que l'auteure universalise cet endroit, qui pourrait se situer n'importe où.

Donc, après ce bref passage à La Butte, Paule se déplace vers la rue la Poudrière, qui se situe au centre de la capitale. Encore une fois, il n'y a que peu d'informations ou de descriptions. La rue est simplement décrite comme « un monde clos » ${ }^{153}$ «où jadis résonnaient les bruits de ferraille d'un chantier de réparation de bateaux, [et qui] contient des enclaves et des tributaires que fréquentent encore les mains flairant la chair $^{154}$. Pourtant d'après Françoise Lionnet, la rue la Poudrière réfère à une rue réelle de l'ancien quartier de Port-Louis. Jusqu'à 1950, cette rue était fréquentée par les prostituées et contenait des rangées de maisons closes bien connues des marins, touristes et bourgeois locaux ${ }^{155}$.

\footnotetext{
${ }^{149}$ Larousse, « Le Port », http://www.larousse.fr/dictionnaires/francais/port/62676?q=le+port\#61970, consulté le 3 mai 2013.

${ }^{150}$ Ananda Devi, Rue la Poudrière, Abidjan, Nouvelles Editions Africaines, 1988, p. 22.

${ }^{151}$ Ivan Seidl, « Aspects de l'espace dans le roman français moderne », www.phil.muni.cz/plonedata/wurj/erb/.../8seidl-74.rtf, consulté le 23 avril 2014, p. 124.

${ }^{152}$ Ananda Devi, Rue la Poudrière, Abidjan, Nouvelles Editions Africaines, 1988, p. 41.

${ }^{153}$ Ibid., p. 126.

154 Ibid., p. 121.

155 «Refers to a very real street in an old neighbourhood of the capital city Port-Louis. Until 1950s this street was frequented by prostitues and lined with maisons closes [...] well known to visiting sailors,
} 
Un autre endroit, plutôt fluvial que terrestre, qui tient une place importante dans la vie de Paule, est la Grande Rivière Nord Ouest. Il est à noter que Paule décrit cette rivière à deux moments bien différents de sa vie. Adolescente, elle y a vu les débris jetés par les usines : «dans la Grande Rivière Nord Ouest parmi les canards blancs et les feuilles d'aloès pourries et les déchets d'usine ${ }^{156}$. Jeune adulte, Paule pense à son amie Aline, morte. N'étant auparavant qu'une coulée d'eau sale, cette rivière prend le visage d'un meurtrier et devient la source de la mort de son amie Aline : « elle est étalée dans la boue, maculée de la tête aux pieds par tout ce qui pollue la Grande Rivière Nord Ouest ${ }^{157}$. Lieu du suicide d'Aline, cette rivière est à l'image de la dégradation subie avec le temps et dont les canards souffrent :

Ils ne dégagent plus la même lumineuse gaieté. Quelque chose les a happés dans le courant primaire de la vie, la pollution les a touchés du doigt, un souffle de mort imprègne leurs plumes les lestant d'une pesante humidité ${ }^{158}$.

La toponymie des lieux met en place l'univers de Paule et le situe à Port-Louis à l'île Maurice. Parallèlement, les descriptions plutôt générales des lieux donnent un caractère universel à ces endroits.

\section{Flore et faune dans Rue la Poudrière}

Ayant localisé l'univers de Paule, notre étude peut désormais se focaliser sur la flore et la faune, et en particulier sur les insectes et reptiles qui fréquentent les différents lieux qu'habite Paule, ainsi que sur les plantes médicinales de sa mère Marie. Paule est entourée des plantes cueillies par celle-ci. Elles peuvent être guérisseuses ou mortelles, utilisées par Marie pour soi-disant guérir les maux tels que «syphilis, stérilité, paralysie, impotence, avortement, poison... » ${ }^{159}$. Ces plantes, dont ni la provenance ni les noms ne sont donnés, servent à faire de la tisane : «au plafond, comme une jungle odorante et crissante de vies multiples et pleines d'ombres, pendaient des dizaines d'espèces de feuilles et de plantes, de couleurs diverses et

tourists and local bourgeois », Françoise Lionnet, «Evading the Subject: Narration and the City in Ananda Devi's Rue la Poudrière », Postcolonial Representations: Women, Literature, Identity, Ithaca, Cornell University Press, 1995, p. 56.

${ }^{156}$ Ananda Devi, Rue la Poudrière, Abidjan, Nouvelles Editions Africaines, 1988, p 27.

${ }^{157}$ Ibid., p. 137.

158 Ibid., p. 135.

${ }^{159}$ Ibid., p. 13. 
d'apparence étrange ${ }^{160}$. Que ce soient des plantes à goût doux ou amer, elles peuvent en effet être soit tropicales, soit venir d'ailleurs.

Un autre élément majeur du texte est l'allusion aux insectes et aux petits reptiles qui partagent l'environnement de Paule, aux Dockers' Flats ou dans la maison close : « les lézards grignotant l'air de leurs cordes vocales, brefs et gris avec leurs petits yeux noirs comme des billes, et qui peuvent rester immobiles [...] et les cancrelats que j'ai toujours connus $[\ldots]$ ceux-ci sont gros, bien nourris, et ils volent ${ }^{161}$. Le lézard, un des reptiles les plus envahissants à l'île Maurice, y a été introduit dans les années $1700^{162}$. L'espèce la plus commune, de couleur grise, vit dans des maisons, exactement comme Paule le suggère. La blatte, plus communément appelée cancrelat à l'île Maurice, vit surtout dans les maisons et a même la capacité de voler dès qu'elle atteint l'âge adulte, exactement comme cela est décrit dans le roman. Rue la Poudrière fait aussi mention des arachnéens et de rongeurs qui se trouvent souvent dans les maisons à l'île Maurice surtout dans les régions chaudes : «cette maison [la maison close] aux plafonds bas et moisis, aux cloisons rongées par les rats et les termites $»^{163}$.

Tout au long du roman, Paule, elle-même, est comparée à des insectes, reptiles ou animaux de petite taille qui sont faciles à éliminer ou intimider : «c'est moi le chien du mendiant qui a oublié d'aboyer [...] comme un rat pris au piège, je sortais de ma cachette ${ }^{164}$, ou encore «Paule, jouait l'oisillon du nid ${ }^{165}$. L'animal peut être agressif : «je me suis vue telle un petit animal féroce, prêt à déchirer et à éventrer sa proie, qu'elle soit souris, lézard ou enfant écarquillée ${ }^{166}$. Paule est ainsi comparée au chien, au rat, à l'oiseau, à la souris ou au lézard ; des espèces qui se trouvent un peu partout dans le monde. La mère de Paule est, elle, comparée à une poule : «Marie a une petite tête à crête de poule noire ${ }^{167}$. Or, la poule noire, associée au maléfique, à l'invocation occulte et à la sorcellerie, est connue dans le monde entier. Cette image suggère que Marie aussi est associée à la magie noire et au malheur. Mallacre, lui, est comparé à un serpent énorme : «Mallacre est un serpent, il se glisse souplement entre les doigts de Marie, il est cobra, non attendez, il est anaconda [...] il est gigantesque, il

\footnotetext{
${ }^{160}$ Ibid., p. 57.

${ }^{161}$ Ibid., p 105.

162 Anonyme, « Mauritius Reptiles »,

http://www.mauritius-reptiles.com/the-reptiles-of-mauritius/introduced-reptiles/house-geckos/, consulté le 21 février 2013.

${ }^{163}$ Ananda Devi, Rue la Poudrière, Abidjan, Nouvelles Editions Africaines, 1988, p.172.

${ }^{164}$ Ibid., p. 3.

165 Ibid., p. 15

${ }^{166}$ Ibid., p. 76.

${ }^{167}$ Ibid., p. 105.
} 
ouvre une gueule immense ${ }^{168}$. L'anaconda, serpent non venimeux originaire de l'Amérique du Sud, est considéré comme un des plus gros serpents du monde tandis que le cobra, originaire de l'Asie, est un serpent agressif dont le venin est mortel. Mallacre est donc dépeint comme un être agressif et dangereux. Si d'un côté il est comparé à ces serpents dangereux, de l'autre côté, il est associé à de grands prédateurs féroces qui sont toujours à la recherche de proies faciles : «sa gorge épaisse comme une gorge de buffle $»^{169}$, « ses yeux fauves $»^{170}$, « ses yeux jaunes de tigre, [...] sa peau rugueuse et épaisse comme celle d'un animal, Mallacre était un lion ou un tigre pleinement grandi ${ }^{171}$. Ces animaux sont ici représentatifs de Mallacre et Marie, qui nuisent et font du mal à Paule.

Par ailleurs, nous observons que certains animaux ont un caractère mythique. Ainsi, « le véritable coq batailleur ${ }^{172}$ relié aux rites vaudou dans le monde entier est assimilé à Mallacre, fait ressortir sa virilité, sa forte personnalité et son pouvoir maléfique. Il est aussi comparé au tigre, qui, dans la mythologie japonaise est le dieu du tonnerre et connu pour sa force. De plus, dans la mythologie chinoise, cet animal est l'un de ceux qui représentent la théorie des cinq éléments. Autant de cas qui montrent à quel point ces comparaisons à caractère mythique dépassent le cadre local auquel sont associés les insectes et les lézards et universalisent la description de l'île Maurice en particulier.

\section{Une description spatiale à la fois locale et universelle dans Rue la Poudrière}

En somme, dans Rue la Poudrière, si les lieux sont localisés, leur description s'avère plutôt universelle. De plus, la flore, la faune, les insectes ou reptiles cités ne sont pas nécessairement locaux et renvoient aussi à l'ailleurs. Ce qui ressort finalement de cette analyse est que même si Rue la Poudrière contient sans aucun doute des références toponymiques ou géographiques locales, d'autres mentions relèvent d'une réalité plus générale, presque mythique. Tout le roman, centré sur la ville de Port-Louis, « restitue une perception diachronique de la réalité ${ }^{173}$ mauricienne puisque cette ville a vu et connu tant de changements et d'évolution économique aussi bien que sociale, et

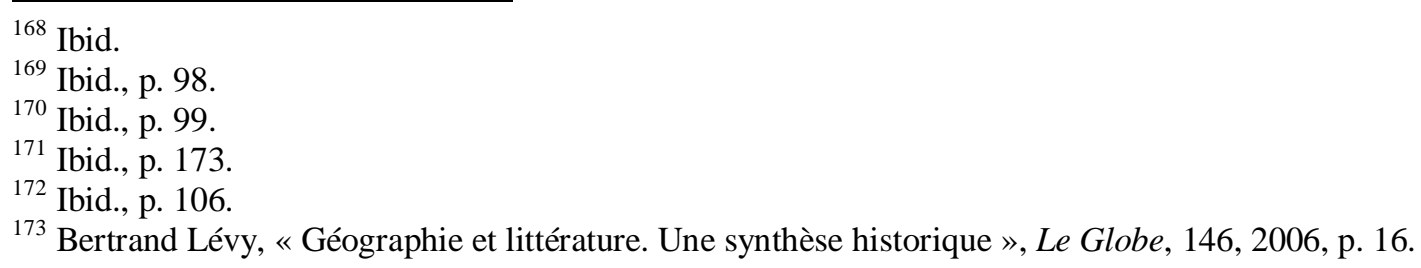


agit comme un «marquage symbolique [...] qui tisse une trame de lieux de mémoire ${ }^{174}$. De cette manière le lecteur mauricien ou étranger est en mesure d'appréhender le monde de Devi qui met « la fiction [...] au service de la réalité ${ }^{175}$ pour ancrer son histoire à Maurice, un lieu réel, tout en créant des personnages aux allures plus universelles.

\section{Le Voile de Draupadi}

Des trois textes à l'étude, c'est Le Voile de Draupadi qui offre le plus de détails en ce qui concerne la description des lieux, de la faune et la flore, d'autant plus que la nature représente un aspect important pour la protagoniste. A part ces informations géographiques et toponymiques, il s'y trouve aussi des données sur la formation géologique de l'île, sur les différentes saisons et surtout sur l'impact qu'ont la faune et flore sur la vie des personnages.

Pour ce qui est de ce roman, il existe jusqu'ici peu de recherches faites sur les aspects toponymiques et géographiques. Jean-Claude Medjo, pour sa part, fait référence à Port-Louis et avance que cette ville, qui représente «l'épuisement [...] l'étouffement [...] la dislocation mentale [et la] disparition physique des personnages » ${ }^{176}$, ressemble à, et rejoint «le destin de toutes les villes modernes ${ }^{177}$. Rohini Bannerjee consacre également quelques lignes de son travail au port et note précisément comment les villages et bâtiments érigés dans l'île sont bien différents des villages du Tamil Nadu qu'ils ont quittés, ajoutant que c'est ce dont la plupart des Indiens se rendent compte en arrivant de l'Inde à l'île Maurice ${ }^{178}$.

D'autres critiques se sont intéressés à l'importance de l'océan dans Le Voile de Draupadi. Ainsi, Bragard considère que « la traversée transocéanique ${ }^{179}$ est un lieu de

\footnotetext{
${ }^{174}$ Ibid., p. 41.

${ }^{175}$ Névine El Nossery, Témoignages fictionnels au féminin. Une réécriture des Blancs de la guerre civile algérienne, New York, Rodopi, 2012, p. 71.

${ }^{176}$ Jean Claude Abada Medjo, «Poétique de la ville dans l'œuvre d'Ananda Devi », http://revel.unice.fr/loxias/index.html?id=7044, consulté le 17 janvier 2013.

177 Ibid.

178 «Upon arriving in Mauritius from India [...] many Indians realize that the villages they left back in Tamil Nadu are quite different from the urban port built in Port-Louis, Mauritius », Rohini Bannerjee, «The Kala Pani Connection: Francophone Migration Narratives in the Caribbean Writing of Raphaël Confiant and the Mauritian Writing of Ananda Devi », Anthurium: a Caribbean Studies Journal, 7, 2009, p. 6.

${ }^{179}$ Véronique Bragard, «Cris des femmes maudites, brûlures du silence, la symbolique des éléments fondamentaux dans l'œuvre d'Ananda Devi », Notre librairie, 142, 2000, p. 66.
} 
mort et de renaissance, d'unité et d'incertitudes ${ }^{180}$, et que la mer constitue un leitmotiv de la traversée de l'océan Indien par les travailleurs engagés. Cette mer qui « lie et délie, détruit et renouvelle, évoqu[ant] à la fois incertitude et éternité Elle devient un lieu de mort et de renaissance, d'unité et d'incertitudes ${ }^{181}$. Rohini Bannerjee et Véronique Bragard remarquent combien cet océan, qui sépare l'Inde de l'île Maurice et qui est souvent nommé «Kala Pani ${ }^{182}$ ou «Eaux obscures » ${ }^{183}$, suggère la souffrance des Indiens qui ont traversé cette grande étendue marine pour aller travailler dans les champs de canne de l'île Maurice.

De plus, Véronique Bragard pense que la mer est personnifiée dans le roman et que Devi « fait sans cesse dialoguer l'eau et le feu dans de nombreuses oxymores ${ }^{184}$. La présence de la mer et du feu sont des éléments très présents dans Le Voile de Draupadi :

L'eau, le feu et la terre, éléments et symboles fondamentaux par excellence, apparaissent dans ces trois romans [Le Voile de Draupadi, Moi, l'interdite et L'Arbre fouet] comme des leitmotiv qui révèlent à la fois le mysticisme et l'héritage hindou de l'auteur ${ }^{185}$.

Puisque ces études survolent la question spaciale, nous proposons d'examiner en détail les divers endroits de l'île Maurice mentionnés dans le roman pour en dégager le degré d'authenticité avant d'étudier l'importance de la nature.

\section{Géographie et toponymie dans Le Voile de Draupadi}

Pendant son adolescence, Anjali, passant la plupart de ses vacances chez son oncle Sanjiva, à Constance-la-gaieté, donne beaucoup de détails topographiques décrivant les différents reliefs de ce village. Situé dans l'est de l'île, ce petit village

\footnotetext{
${ }^{180}$ Ibid., p. 191.

${ }^{181}$ Ibid.

${ }^{182}$ Rohini Bannerjee, «The Kala Pani Connection: Francophone Migration Narratives in the Caribbean Writing of Raphaël Confiant and the Mauritian Writing of Ananda Devi », Anthurium: a Caribbean Studies Journal, 7, 2009, p. 1-11.

${ }^{183}$ Véronique Bragard, «Eaux obscures du souvenir. Femme et mémoire dans l'œuvre d'Ananda Devi », Convergences and Interferences: Newness in Intercultural Practices. Ecrire d'une nouvelle ere/aire, Amsterdam et New York, Rodopi, 8, 2002, p. 187-199.

${ }^{184}$ Ibid., p. 191.

${ }^{185}$ Ibid., p. 70.
} 
agricole réel est habité en grande partie par les travailleurs des champs de canne, ce qui aide à en dresser un portrait réaliste ${ }^{186}$.

Même si le nom de l'endroit où habitent les parents d'Anjali n'est pas mentionné dans le roman, la narratrice précise qu'ils habitent tout près de l'autoroute aux «frontières grouillantes de Quatre-Bornes et de Rose-Hill » ${ }^{187}$. Il est intéressant de noter à ce propos que l'île Maurice compte une seule autoroute, construite dans les années 1960, et qui relie le Sud-Est au Nord ${ }^{188}$. Connus pour leurs commerces, QuatreBornes et Rose-Hill sont deux villes résidentielles qui se situent dans le district de Plaines Wilhems, sur le plateau central de l'île. En ne précisant pas le nom du lieu de résidence des parents d'Anjali, Devi évite un ancrage spécifique reconnaissable ou vérifiable, fictionnalisant certains aspects de son histoire ${ }^{189}$.

Lors d'une longue promenade en voiture avec son mari, Anjali arrive au sudouest de l'île, où elle raconte la naissance de l'île et accentue son origine volcanique :

On ne peut pas aller plus loin. L'océan est déjà là et le collier de récifs s'interrompt ici pour laisser pénétrer des formes liquides (auxquelles) on peut à peine leur donner le nom de vagues. [...] Les gerbes d'eau gravissant nerveusement les rochers [qui étaient] là au tout début, [qui] ont connu un frémissement des gaz et des énergies comprimés sous la voûte terrestre, [qui] ont connu la fureur des explosions telluriques, les coulées de lave qui, à chaque refroidissement, se solidifiaient et devenaient un sol, un espace et une aire ${ }^{190}$.

\footnotetext{
186 « Furthermore, characters travel through countries, regions and cities, or dream of the ocean [...] or remember places of their childhood [...]. Consequently, we are dealing with heterogeneous data concerning topographical elements (such as building, town, country, continent etc) and basic geometrical elements [...] that are described by supplementary attributes », Anne-Kathrin Reuschel, Lorenz Hurni, «Mapping Literature: Visualisation of Spatial Uncertainty in Fiction », The Cartography Journal, 48, 2011, p. 295.

${ }^{187}$ Ananda Devi, Le Voile de Draupadi, Paris, L'Harmattan, 1993, p. 120.

188 Auguste Toussaint, Port-Louis. A Tropical City, Londres, Allen and Unwin, 1973, p. 120.

189 «The distinction of these literary spatial entities is not realized by the topographical objects to which they refer, but by the function the entity is used for within fiction [...] For instance, a place of action can be, on one hand, inside of a building or a city, but on the other hand, this building or city might only be roughly located somewhere within a given region », Anne-Kathrin Reuschel, Lorenz Hurni, « Mapping Literature: Visualisation of Spatial Uncertainty in Fiction », The Cartography Journal, 48, 2011, p. 294.

${ }^{190}$ Ananda Devi, Le Voile de Draupadi, Paris, L'Harmattan, 1993, p. 82.
} 
Anjali précise que « remontant vers les hauts plateaux » ${ }^{191}$ «vers les hauts contreforts de Moka ${ }^{192}$, le couple a continué sa route et a «longé la montagne Ory, avec ses amples terrassements plantés de cannes ${ }^{193}$ qui sont localisés au pied de la chaîne de Moka $^{194}$. Anjali, la narratrice, dévoile avec ce voyage, la géographie de l'île Maurice d'est en ouest en passant par le sud mais explore très peu la partie nord de l'île.

Le roman dépeint également le cœur de l'île, où se trouve «Port-Louis, la capitale politique et économique de l'île Maurice ${ }^{195}$ et où se trouvent les Salines, où Anjali part se ressourcer ${ }^{196}$. Ce lieu est perçu comme un lieu «rescapé des bouleversements industriels de Port-Louis ${ }^{197}$; dans la réalité, les Salines sont un quartier résidentiel dans les faubourgs du sud de la capitale. Anjali mentionne aussi le «temple de Mariammen » ${ }^{198}$, qui est l'un des premiers temples tamouls à Maurice. Quand Anjali part retrouver son amie Fatmah, elle fait une description assez détaillée de la partie de la ville où se situent les quartiers chinois et musulman :

Fatmah habitait le quartier musulman de Port-Louis. Il n'y régnait pas cette atmosphère dense, lourde et odorante du quartier chinois [...] la porte de la mosquée était ouverte, et les hommes en y entrant, délaissaient sans crainte leurs sandales à l'entrée [...] des cours rigoureusement fermées, interdites au regard des passants et des curieux, s'échappaient des effluves riches de parfum d'épices ${ }^{199}$.

Comme pour le roman Rue la Poudrière, Le Voile de Draupadi contient donc des informations toponymiques très réalistes qui aident le lecteur à se situer dans l'île et la capitale. Par conséquent, le décor élaboré dans le roman joue « un rôle primordial [et crée] autour des figures principales du roman, un cadre qui leur [est] propre et à travers

\footnotetext{
${ }^{191}$ Ibid., p. 152.

192 Ibid., p. 119.

193 Ibid.

${ }^{194}$ Situé dans le district de Moka, de l'autre côté de la chaîne des montagnes qui sépare Port-Louis et Moka, la montagne Ory est connue pour ses température modérées.

195 Jean Claude Abada Medjo, « Poétique de la ville dans l'œuvre d'Ananda Devi », http://revel.unice.fr/loxias/index.html?id=7044, consulté le 17 janvier 2013.

${ }^{196} \mathrm{Si}$ le plateau central est connu pour sa température basse et son humidité, le Nord de l'île et PortLouis, sont connus pour leur climat chaud et sec.

${ }^{197}$ Ananda Devi, Le Voile de Draupadi, Paris, L'Harmattan, 1993, p. 112.

198 Ibid., p. 151.

${ }^{199}$ Ibid., p. 101
} 
[...] duquel se manifeste leur personnalité ${ }^{200}$. Ainsi, les descriptions géographiques et toponymiques ne contribuent pas seulement à «localiser la scène ${ }^{201}$ mais « [elles] institue[nt] autour des personnages et de l'action un cosmos nécessaire, cohérent, qui prolonge et représente dans le visible, comme fait un emblème ou une figure, certaines données invisibles qu'il rend ainsi plus insistantes et plus aisément perceptibles au lecteur $»^{202}$.

\section{Nature, flore et faune dans Le Voile de Draupadi}

L’importance de la nature dans la vie des personnages est indéniable. En effet, ces derniers se retrouvent très souvent hors de leur lieu d'habitat, au cœur de la nature. Pour se ressourcer, ils font des randonnées, ou escaladent la montagne où ils tiennent des séances de méditation. Anjali exprime ce besoin de se retrouver en pleine nature à maintes reprises, mais surtout lorsqu'elle escalade le Pieter Both, connu pour être la deuxième plus haute montagne de l'île, et pour ressembler à une tête humaine ${ }^{203}:$ «à mesure que je montais, je me sentais devenir ivre, folle. Quelque chose se mettait à vibrer en moi » ${ }^{204}$. C'est lors de son ascension qu'Anjali réalise à quel point la nature la rend heureuse, et du fait qu'elle est amoureuse de Dev :

Le Pieter Both aux formes sculpturales et ailées, [...] elle n'était pas bien grande, cette montagne, mais les formes tailladées de cette chair volcanique pétrifiée dans d'impossibles contorsions la rendaient menaçante $[\ldots]$ ce petit joyau de verdure qu'était l'île, avec ses visages incessantes, ses métamorphoses surnaturelles. De la montagne s'élevait le souffle tumultueux de ma première

\footnotetext{
${ }^{200}$ François Richard, « Le décor romanesque », Etudes françaises, 8, 1972, p. 346.

201 Ibid., p. 347.

202 Ibid., p. 348.

203 «Il y a une légende bien connue à l'île Maurice, supposément rattachée au Pieter Both : les bergers faisaient brouter l'herbe à leurs moutons sur les flancs de la montagne. Ces bergers étaient souvent avertis par les anciens d'éviter d'emmener leur troupeau de l'autre côté de la montagne. Mais un beau jour un des bergers a dû transgresser cette règle pour aller récupérer un de ses moutons qui était parti de l'autre côté du Pieter Both. Dès que le berger est arrivé à l'autre bord de la montagne, sa tête s'est transformée en pierre par les fées », Shaen Adey, Fiona McIntosh, Sept jours à L'île Maurice et Rodrigues, Le Cap, New Holland Publishing Limited, 2004, p. 25.

${ }^{204}$ Ananda Devi, Le Voile de Draupadi, Paris, L'Harmattan, 1993, p. 20.
} 
émotion véritable, le premier sentiment qui me poussât vers un homme autre que Shyam ${ }^{205}$.

Par ailleurs, Anjali fait mention du climat varié et variable de l'île:

L'hiver tropical est froid et lumineux, à la fois coloré et très blanc. Le jour se contredit lui-même plusieurs fois [...], changeant d'humeur et d'aspects. Sur le rebord de la fenêtre, un moineau s'est blotti dans un rectangle de soleil en gonflant ses plumes pour se protéger du froid ${ }^{206}$.

Anjali part ensuite au plateau central où :

Il pleut ce matin comme il ne peut pleuvoir que sur les hauts plateaux, ce crachis constant et froid qui jette une poussière de rien sur le pare-brise de la voiture, puis d'un coup, sans prévenir, se met à le noyer. Les nuages ont l'air d'être descendus sur la ville, mais ce n'est que le brouillard du matin, qui se dissipera tout à l'heure lorsqu'il aura achevé son œuvre de démantèlement ${ }^{207}$.

Ceci montre à quel point Anjali est proche de la nature et de son milieu. Elle se remémore souvent des journées passées au cœur de la nature, en compagnie de son frère et de sa cousine, Vasanti :

Comment pourrais-je jamais oublier les jours passés làbas [à Constance-la-Gaieté] ? Le réveil, au matin, avec des chants d'oiseaux entrant par la fenêtre ouverte, les rosiers filants égratignant impérieusement les volets verts pour nous commander de sortir. Un bruit d'eau tout près, et ces parfums de la terre, tièdes, épicés, mélanges de thym et de menthe, de coriandre et de camphre ${ }^{208}$.

Déjà adolescente, Anjali se sentait en parfaite union avec la nature environnante. Adulte, elle continue d'avoir besoin de ses vibrations positives, surtout pour gérer tout

\footnotetext{
205 Ibid., p. 21.

${ }^{206}$ Ibid., p. 17.

${ }^{207}$ Ibid., p. 39.

${ }^{208}$ Ibid., p. 44.
} 
le stress occasionné par la mort de son fils : «à présent, assise sur la balançoire, frôlée par les feuilles comme par des lèvres humides [...] je me sens enfin libre de pleurer $»^{209}$. De fait, Anjali donne toute une liste de plantes tropicales, au point de constituer une sorte de guide botanique : «le buisson de frangipanier, les jacarandas et les flamboyants $»^{210}$ ou encore, «des fibres de coco [...] les bananiers [...] ces fruits des bois $[\ldots]$ papayes rouges $[. .$.$] des patates douces, du manioc { }^{211}$. Anjali fait également référence aux diverses plantations de la vallée, au nord de l'île, qui est supposément l'endroit idéal pour faire pousser toutes sortes de fleurs et d'arbres fruitiers :

La végétation dans la vallée avait toujours été plus dense que dans le village. Les papayers et les badamiers poussaient à l'état sauvage [...] les enfants aimaient aussi beaucoup ces fruits des bois [...] il y avait des buissons de jujubes [...] nous déterrions des patates douces, du manioc, des tubercules inconnus ${ }^{212}$.

Il est question aussi, bien sûr, de la canne à sucre dont la culture est répandue un peu partout dans l'île :

Très hautes, prêtes à être coupées, celles-ci arboraient cette glorieuse et rare couleur d'émeraude [...] et les tiges de canne brune au cœur des feuilles étaient épaisses et riches en sucre, [...]. Un des champs avait déjà été coupé et nettoyé, et la terre chaude, remuée, avait une bonne odeur rouge, une odeur de fertilité 213 .

Contrairement aux descriptions de la végétation qui mettent en place l'univers mauricien des personnages, les références à la faune sont en général des métaphores. Anjali est perçue comme lionne, chienne ou « mère-louve » ${ }^{214}$, des animaux intimidants mais qui ont surtout un grand instinct maternel :

\footnotetext{
${ }^{209}$ Ibid., p. 32.

${ }^{210}$ Ibid., p. 74.

${ }^{211}$ Ibid., p. 34.

212 Ibid.

213 Ibid., p. 119.

${ }^{214}$ Ibid., p. 88.
} 
Je pourrais y retrouver, tel un chien flairant quelque trace, la présence de mon enfant ${ }^{215}$;

Réintégrant ma peau de mère et de lionne, armée d'un instinct aussi féroce qu'il est primitif ${ }^{216}$;

La lionne ne capitule qu'à la dernière extrémité. Elle surveille avec hargne, avec méfiance, le sommeil de son petit. Ses yeux furtifs glissent passagèrement sur les présences intruses terrées à l'orée du bois. Puis, sentant leur terreur devant sa force, son innombrable pouvoir, rassurée qu'ils ne se rapprocheront pas pour lui enlever son fils, elle se retourne pesamment et pose définitivement sur lui son regard ${ }^{217}$.

Par contre, Vasanti est associée au milieu aquatique et aux poissons : «elle luisait, glissante et brune comme un poisson de l'étang [...] on ne voyait que Vasanti qui nageait, une longue chevelure noire, dansante comme une algue [...] je m'approchai, je vis qu'il n'y avait que la chevelure d'algue $»^{218}$. Dev est, quant à lui, comparé à «l'ogre du petit poucet ou [au] loup du chaperon rouge $»^{219}$ par Anjali. Il est intéressant de noter que la plupart de ces comparaisons animalières font allusion à de gros animaux terrestres peu faciles à maîtriser, ou à des animaux aquatiques insaisissables qui peuvent se faufiler facilement.

Il est clair que l'histoire se déroule à l'île Maurice puisque l'ensemble des espèces végétales dont il est question sont tropicales et que les noms des villes, villages, montagnes soulignent le fait qu'il s'agit bien de l'île Maurice. Par contre, la comparaison des personnages à des animaux communs ou mythiques tels le loup, la lionne, le chien, les poissons, relève de l'universel.

\footnotetext{
${ }^{215}$ Ibid., p. 42.

216 Ibid., p. 50.

${ }^{217}$ Ibid., p. 171.

${ }^{218}$ Ibid., p. 33

${ }^{219}$ Ibid., p. 97.
} 


\section{Un décor local et réaliste dans Le Voile de Draupadi}

Il ressort que Devi a intégré la couleur locale ${ }^{220}$ de façon très précise dans le roman et construit un décor mauricien réaliste qui rend l'histoire plus vraisemblable. De ce fait, les lecteurs n'ont pas besoin de connaître l'île Maurice pour imaginer l'univers des personnages ${ }^{221}$. Très vraisemblable, le décor présente également une «fonction esthétique ${ }^{222}$ qui « s'écarte [...] de sa mission purement réaliste ${ }^{223}$ avec certaines métaphores animalières identifiant le tempérament des personnages. Pour Devi, il est important de mélanger l'universel au local : «parler de Maurice mais [aussi] du monde en même temps [dans le but, non pas] d'écrire pour son petit cercle mais pour tout le monde $»^{224}$. Pourtant, ce roman, fortement ancré dans le milieu mauricien, reste plus local qu'universel ${ }^{225}$ en ce qui concerne sa dimension spatiale.

\section{Le Sari vert}

Il est apparent dès la première lecture que Le Sari vert présente peu d'éléments toponymiques et encore moins de détails sur la faune et la flore. Le peu d'informations présentes sur la faune et la flore est transmis par les trois personnages principaux du roman; le docteur Bissam, Kitty et Malika. Les lieux donnés sont rares et l'environnement passe au second plan. Ce sont plutôt les sentiments et l'état d'âme du vieil homme, le docteur Bissam, alias Dokter Dieu, qui est en phase terminale d'un cancer, qui importent. Pour le vieil homme alité, son environnement n'a presqu'aucun intérêt.

\footnotetext{
220 «Couleur locale henceforth stands for the typical features of a historic setting or a given locale, or can incorporate both », Vladimir Kapor, «Couleur locale. A pictoral term gone astray? », A Journal of Verbal/Visual Enquiry, 25, 2008, p. 27.

${ }^{221}$ «Readers need not be familiar with the reality that the texts is about in order to believe it true », Michael Riffaterre, Fictional Truth, London, The John Hopkins University Press, 1990, p. 8.

${ }^{222}$ Roland Barthes, «L'Effet de réel », Communications, 11, 1968, p. 85.

${ }^{223}$ François Richard, « Le Décor romanesque », Etudes françaises, 8, 1972, p. 346.

${ }^{224}$ Le Mauricien, «Entretien avec Ananda Devi, "Il y a une scission de moi-même ; dans mes livres j'ose tout" ",

http://www.lemauricien.com/article/ananda-devi-ecrivain\%C2\%A0-\%C2\%AB\%C2\%A0il-y-scissionmoi-meme\%C2\%A0-mes-livres-j\%E2\%80\%99ose-tout\%C2\%A0\%C2\%BB, consulté le 21 décembre 2012.

${ }^{225}$ « These are the two [en faisant référence à L'Arbre fouet et Le Voile de Draupadi] where I went very deep, both in trying to explore the inner depths or the philosophy of religion, or mysticism rather, and then at the same time condemning the ritualistic fanaticism. So I think, because these two books are very grounded in Mauritian society, they were less universal and much more about the actual real society », Peter Hawkins, « An Interview with Ananda Devi », Wasafiri, 26, 2011, p. 13.
} 


\section{Géographie et toponymie dans Le Sari vert}

Au début du roman, le vieil homme, seul dans sa chambre, fait allusion à la ville de Curepipe ; aussi connue comme la Ville-Lumière, Curepipe est située au centre de l'île dans les hauteurs. C'est parce que la maison de Kitty est située à Curepipe que le vieil homme commente : «La ville de Curepipe est bien la seule du pays à connaître un automne permanent. Il n'y a qu'elle pour vivre dans un endroit qui refuse aussi obstinément le soleil tropical $»^{226}$.

Par ailleurs, le docteur Bissam se souvient qu'étant jeune, il «traversai[t] l'île de nord en sud ${ }^{227}$ pour soigner ses malades, sans pour autant donner d'amples détails sur le Nord de l'île. D'autres régions mentionnées par le docteur Bissam sont PortLouis $^{228}$, Camp Carole ${ }^{229}$, Camp Kenya ${ }^{230}$. Ces deux derniers sont deux petits villages situés dans le sud-est de l'île. Mention est aussi faite de Mahébourg ${ }^{231}$, petite ville côtière dans le sud-est de l'île. Il se rappelle aussi les marais salins à Tamarin ; endroit côtier dans l'ouest de l'île, connu pour être le lieu le plus sec et le plus chaud. C'est aux Salines de Tamarin, que le vieil homme revoit souvent sa femme : «Nous marchons dans les salines [...] étendues plates, carrés de mer s'évaporant lentement pour ne laisser qu'un résidu de sel d'un blanc insoutenable ${ }^{232}$.

Dans les souvenirs du vieil homme, Malika, parlant à sa mère, oppose Curepipe à Case Noyale ; village côtier dans l'ouest de l'île où elle travaille comme institutrice, et où l'activité principale des villageois est la pêche : « il fait si chaud à Case Noyale, tu vois, pas comme à Curepipe, froidure humide, moisissures glacées, non, là-bas c'est le chaud du vent et de la terre qui monte, seulement un peu déblayé par le vent marin ${ }^{233}$. Kitty, elle, fait allusion à la péninsule du Morne, une falaise située dans l'extrême sudouest de l'île bien connue pour sa légende des esclaves malgaches et africains marrons qui s’y étaient réfugiés ${ }^{234}$.

Ces lieux divers nommés placent l'histoire du docteur Bissam et de sa famille à l'île Maurice.

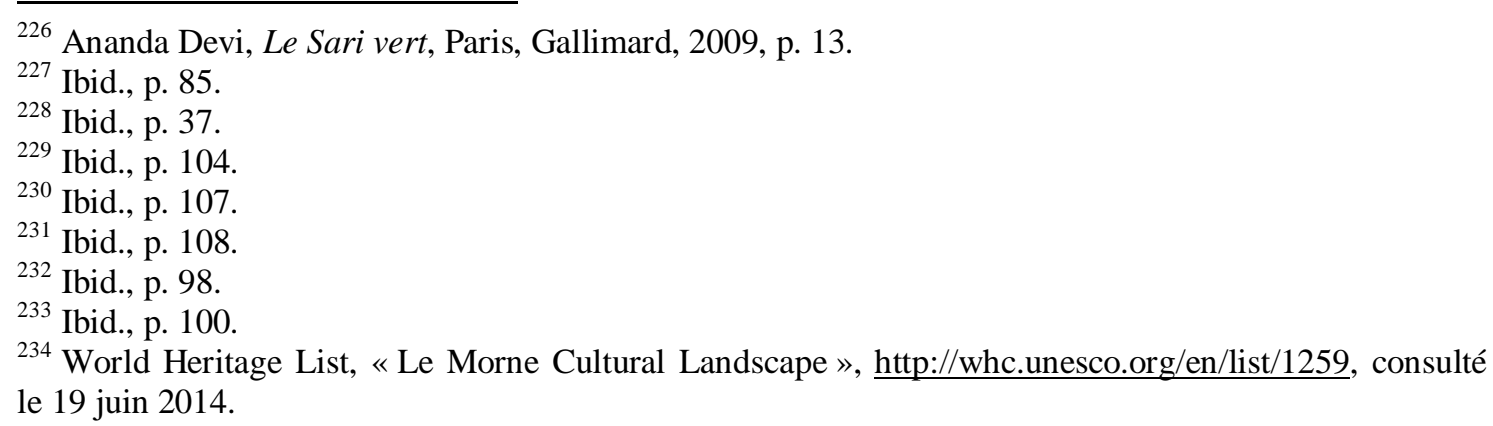




\section{Flore et faune dans Le Sari vert}

Il n'existe qu'une seule et brève mention de la flore faite par le vieil homme. Il s'agit «des enfilades de cannes, le trésor de l'île » ${ }^{235}$, la seule plante que le docteur arrive à apprécier.

Il n'y a pas de détails non plus sur les animaux présents sur le sol de cette île, seulement de brèves mentions d'animaux ou d'oiseaux montrant que le docteur Bissam trouve la nature dérangeante, et menaçante :

Les bengalis fous de pluie ont repris leur chorale. Ils ne me laissent pas en paix. Chaque fois que je commence à m'endormir, on dirait que tous les oiseaux de la terre le savent. Après ce sera les crapauds, ensuite les chiens. Une symphonie animalière, mais sans harmonie aucune [...] Ceux qui ont les yeux ouverts savent, au contraire, que ce sont les hommes qu'il faut protéger de la nature [...] les deux corbeaux femelles, là-bas, récitant leur litanie de rancunes, sont aussi une menace ${ }^{236}$.

Cependant, le vieil homme, narcissique et machiste, compare Kitty et Malika au cochon ou à la chienne : «à l'affût comme des chiennes en manque » ${ }^{237}$. Eternellement insatisfait de sa fille, le vieil homme saisit chaque occasion pour verser son venin sur kitty en l'insultant et en la comparant à des bêtes : « Kitty, Kitty, Kitty, viens ma chatte, viens sur les genoux de papa $»^{238} ;$ «chienne $»^{239}$. En fait, le vieil homme montre sa haine et son mépris envers toutes les femmes en les comparant à une vache : «c'est seulement après que je suis allé m'occuper de l'autre vache qui n'a eu, elle, aucun mal à faire sortir l'enfant ${ }^{240}$. Il compare aussi la compagne de Malika, Marie-Rose Patience, à une «vache noire ${ }^{241}$ et fait des allusions dénigrantes vis-à-vis de Malika : «tu disais à maman [kitty] que je ressemblais à un gros cochon ${ }^{242}$ ou encore «cette fille est une tisane d'herbe bourrique. Cueillie là où toute une portée de lapins a

\footnotetext{
${ }^{235}$ Ananda Devi, Le Sari vert, Paris, Gallimard, 2009, p. 85.

${ }^{236}$ Ibid., p. 14-15.

${ }^{237}$ Ibid., p. 80.

${ }^{238}$ Ibid., p. 14.

${ }^{239}$ Ibid., p. 93.

${ }^{240}$ Ibid., p. 90.

${ }^{241}$ Ibid., p. 49.

242 Ibid., p. 47.
} 
pissé ${ }^{243}$. Le jour où Kitty se trouve un mari, ne pouvant digérer cette trahison de sa fille, il n'épargne pas son gendre et le compare à un rat : « le rat, le rat, j'assassine le rat et sa cohorte. Le rat qui m'a tout pris $»^{244}$.

Puisque la perception de l'environnement passe essentiellement par le vieil homme malade cloué au lit chez sa fille à Curepipe, il n'est pas surprenant qu'il se remémore certains épisodes de son passé à Port-Louis, Camp Kenya et Mahébourg qu'il traversait pour aller soigner ses patients. De plus, le vieux médecin, invalide et cloîtré chez sa fille qui s'occupe de lui, perçoit son nouvel environnement avec horreur : « la maison était un enfer. Je levais les yeux et je voyais des toiles d'araignée se balançant au plafond ${ }^{245}$. Les lieux, la faune et la flore sont surtout filtrés par le vieil homme puisque le roman est principalement constitué du monologue du vieux médecin mourant, exactement comme l'explique Ivan Seidl :

Si un récit nous est présenté tout entier au moyen du monologue intérieur, nous ne percevons de la réalité que ce qu'a pu en percevoir tel ou tel personnage puisque nous adoptons son "point de vue". La technique du point de vue ne consiste pas [...] seulement dans le monologue intérieur, mais d'une façon quelconque, elle ne transmet au lecteur que la réalité que perçoit le héros. Le monde extérieur s'incorpore dans le courant de conscience ${ }^{246}$.

\section{Une localisation locale mais une faune et flore universelles dans $\mathrm{Le}$ Sari vert}

Ce qui fait que l'espace mauricien a relativement peu de place dans le texte et que les comparaisons animalières reflètent les attitudes dénigrantes et sexistes du vieux médecin. Par ailleurs, puisque le personnage est cantonné à la maison, son champ visuel est limité alors que l'ouïe acquiert plus d'importance, d'où les allusions aux chants des oiseaux ou au son de la pluie ${ }^{247}$. Si les réminiscences du personnage principal nous emmènent de Port-Louis à Camp Kenya, ou à Mahébourg, c'est une

\footnotetext{
${ }^{243}$ Ibid., p. 74.

${ }^{244}$ Ibid., p. 145.

245 Ibid., p. 126.

${ }^{246}$ Ivan Seidl, « Aspects de l'espace dans le roman français moderne », www.phil.muni.cz/plonedata/wurj/erb/.../8seidl-74.rtf, consulté le 23 avril 2014, p. 127.

${ }^{247}$ « Dans les camphriers, les bengalis fous de pluie ont repris leur chorale. Ils ne me laissent pas en paix. Chaque fois que je commence à m'endormir, on dirait que tous les oiseaux de la terre le savent », Ananda Devi, Le Sari vert, Paris, Gallimard, 2009, p. 14.
} 
excursion limitée et nous n'avons aucune description ni même aucune mention des villages du Nord de l'île.

\section{Localisation spécifique et universalisation dans les trois romans}

L'analyse détaillée des trois romans a montré que les références aux lieux, aux plantes, aux animaux et aux insectes jouent un grand rôle dans Rue la Poudrière et Le Voile de Draupadi mais bien moins dans Le Sari vert où les allusions à la géographie, la faune et la flore n'apparaissent que brièvement.

Nous avons vu que, dans Rue la Poudrière, les lieux tels que l'église MarieReine-de-la-Paix, la montagne des Signaux, la Grande Rivière Nord-Ouest, le Champ de Mars et la cathédrale Saint-Louis, et dans Le Voile de Draupadi, les endroits tels que Quatre-Bornes, Rose-Hill, Plaine Wilhems, Constance-la-Gaiété, Moka, la montagne Ory et le temple Mariammen ancrent l'univers des personnages très précisément à l'île Maurice. La technique de nommer les lieux trouve tout particulièrement sa place dans le plus toponymique des trois textes, Rue la Poudrière, qui donne une idée précise d'endroits et de monuments de Port-Louis. Le Sari vert est également situé à l'île Maurice et fait mention des endroits tels Port-Louis, Curepipe, Mahébourg, les Salines de Tamarin, Camp Kenya, Camp Carole, Case Noyale et Mon Trésor Mon Désert. Pour ce qui est de la flore et de la faune, Le Voile de Draupadi présente toute une panoplie d'espèces végétales et animales endémiques à l'île Maurice qui, selon l'auteure, a été introduit pour satisfaire les éditeurs qui voulaient de longues descriptions de la flore et de la faune mauriciennes pour donner un caractère plus local au roman. Par exemple, il est fait mention du «Paille-en-Queue ${ }^{248}$, du buisson de frangipanier, des jacarandas, des flamboyants, des fibres de coco, des bananiers, des papayes rouges, des patates douces et du manioc. Pour ce qui est du Sari vert, il est question de bengalis, oiseau commun à l'île Maurice, et de la canne à sucre, omniprésente dans l'île. Rue la Poudrière présente toute une liste d'espèces animales, telles que le lézard, le cancrelat, le rat et la termite que l'on trouve communément à l'île Maurice. De la sorte, l'ensemble des références toponymiques et géographiques enracine les histoires et les personnages dans l'île Maurice et renforce «l'effet de réel » dans les trois livres. Le local qui découle de ces allusions aux lieux, à la flore et à la faune propres ou communes à l'île Maurice contribue à la vraisemblance de leur histoire fictive.

${ }^{248}$ Ananda Devi, Le Voile de Draupadi, Paris, L’Harmattan, 1993, p. 18. 
L'auteure, dans un de ses entretiens, constate d'ailleurs que « les personnages que je créais naissaient de mon imaginaire, ou peut-être de rencontres brèves et fulgurantes dans une rue, sans que j'en sache rien de plus, mais les paysages décrits étaient, eux, directement empruntés à la réalité ${ }^{249}$.

Si certains détails localisent et concrétisent l'histoire et l'univers des personnages, d'autres mondialisent le roman. La description faite dans Le Voile de Draupadi du Pieter Both ${ }^{250}$ est un exemple typique d'universalisation. Bien qu'ancrée dans le milieu mauricien par son nom, cette montagne de l'île maurice est décrite comme n'importe quelle montagne volcanique. De par sa description, cette montagne mauricienne acquiert un caractère générique qui contribue au phénomène d'universalisation. Dans Rue la Poudrière, la rue où se trouve la maison close est nommée mais non décrite ; ce qui fait de cette rue de Port-Louis le symbole de toutes les rues du monde fréquentées par des prostituées. De même, la description très réaliste des Dockers' Flats rappelle celle des Dockers' Flats dans d'autres pays quant à son état d'écroulement. De fait, les Dockers' Flats de Port-Louis, comme les Dockers' Flats de Londres, représentent le quartier insalubre et surpeuplé typique où les bâtiments forment un ensemble de minuscules appartements construits en rangées ${ }^{251}$ avec des murs et plafonds noircis avec l'accumulation des impuretés entassées au fil de longues années de négligence ${ }^{252}$. Ces endroits sont tellement négligés et mal construits que les rayons de soleil n’y pénètrent jamais; ces appartements ne reçoivent jamais une bouffée d'air frais et connaissent rarement les plaisirs d'une goutte d'eau fraîche ${ }^{253}$. Cette description des New Victoria Docks à Londres correspond exactement à celle faite par Devi des Dockers' Flats de Port-Louis. En quelque sorte, nous pouvons dire qu'il y a universalisation du quartier des travailleurs portuaires dans le roman, d'autant plus que Devi, prenant des libertés avec la réalité, dépeint des Dockers' Flats délabrés,

\footnotetext{
${ }^{249}$ Indes réunionnaises, «L'Ecriture est le monde, elle est le chemin et le but », entretien avec Ananda Devi, Indes réunionnaises, 2003, http://www.indereunion.net/actu/ananda/intervad.htm. Web, consulté le 22 juillet 2011.

${ }^{250}$ «Elle n'était pas bien grande, cette montagne [Pieter Both], mais les formes tailladées de cette chair volcanique pétrifiée dans d'impossibles contorsions la rendaient menaçante. La montée n'était pas difficile. Très souvent les pentes s'adoucissaient, comme pour nous ouvrir une voie d'accès, et les pierres s'émoussaient. Des parfums inconnus s'évadaient des échancrures, quelque chose de sec et d'enivrant, comme une ancienne ferveur », Ananda Devi, Le Voile de Draupadi, Paris, L'Harmattan, 1993, p. 18.

${ }^{251}$ « The houses are built in rows », Charles Dickens, «Londoners Over the Border », Household Words, 16,1857, p. 241

${ }^{252}$ «Walls and ceiling [...] black with the accretions of filth which have gathered upon them through long years of neglect», Andrew Mearns, The Bitter Cry of Outcast London. An Inquiry into the Condition of the Abject Poor, London, James Clarke, 1883, p. 8.

253 «The sun never penetrates, [...] never visited by a breath of fresh air, and [...] rarely know the virtues of a drop of cleansing water », Ibid., p., 7 .
} 
anciens et dégradés alors qu'ils sont neufs et propres au moment où la famille de Paule y emménage. A en croire Reuschel et Hurni, les espaces dépeints dans un roman peuvent être transformés ou remodelés selon les besoins narratifs ${ }^{254}$; et c'est exactement ce qui se passe dans le roman où Devi peint une réalité décalée dans le temps pour véhiculer l'idée de pauvreté. Les plantes médicinales qu'utilise Marie dans Rue la Poudrière, parce qu'elles ne sont pas nommées, contribuent également à cet effet d'universalisation. Dans Le Voile de Draupadi, ce sont certains oiseaux, tels le moineau ${ }^{255}$ ou le martin ${ }^{256}$, qui donnent la note universelle au roman. Dans Le Sari vert, des espèces animales que l'on trouve partout, telles le cochon, le chien, le lapin ou l'araignée, sont utilisées par Bissam pour dénigrer les femmes de sa famille certes, mais aussi les femmes en général. En outre, les comparaisons et analogies aux animaux mythiques, comme par exemple le lion dans Rue la Poudrière et Le Voile de Draupadi, mettent également en lumière cette tendance vers l'universel, vers l'ailleurs ciblé par Devi qui choisit en universalisant certaines références animalières de dépasser le local. Dans l'hindouisme, le lion est connu pour sa force et est aussi le véhicule de la déesse invincible Durga. Dans la mythologie égyptienne, le lion, qui représente plusieurs dieux et déesses, est vénéré pour sa force. Dans Le Voile de Draupadi, les références au poisson renvoient à Matsya, le Dieu poisson qui est considéré être l'incarnation du Dieu protecteur Vishnu dans la mythologie indienne. Dans Le Sari vert, la vache fait allusion à la déesse hindoue Nandi et au dieu Shiva. Ainsi, toutes ces comparaisons ou métaphores animalières qui renvoient aux mythes évoquent un ailleurs plus universel. De la même façon, les références au Petit Poucet, au Chaperon rouge, à la baleine de Moby Dick ${ }^{257}$ dans Le Voile de Draupadi ramènent aux contes bien connus et font appel à un imaginaire nourri de symbolisme universel. Le fait que les romans de Devi «sont lus partout, [et] que les lecteurs [les] comprennent bien ${ }^{258}$ est bien la preuve qu'un phénomène narratif d'universalisation s'ajoute à l'ancrage local de ses romans, en particulier en ce qui concernent les trois romans à l'étude.

\footnotetext{
254 «Spaces in narrations can be transformed or remodelled by the author; this ranges from simple renaming of places to the combination or relocation of places to totally invented places without an obvious conterpart in the geospace », Anne-Kathrin Reuschel, Lorenz Hurni, « Mapping Literature: Visualisation of Spatial Uncertainty in Fiction », The Cartography Journal, 48, 2011, p. 294.

255 Ananda Devi, Le Voile de Draupadi, Paris, L'Harmattan, 1993, p. 17.

${ }^{256}$ Ibid., p. 18.

257 «Je m'étais promis de lui raconter l'histoire de Moby Dick », Ananda Devi, Le Voile de Draupadi, Paris, L'Harmattan, 1993, p. 42.

${ }^{258}$ Nathalie Philippe, « Explorer l'île pour atteindre l'universel. Retour sur l'œuvre d'Ananda Devi », Les Dépêches de Brazzaville, http://www.brazzaville-adiac.com/medias/dossiertele/suplit7.pdf, consulté le 13 mars 2013.
} 


\section{Partie III}

\section{Dimension temporelle}




\section{Introduction}

Dans ce chapitre, nous visons à examiner les références historiques dans les trois romans pour en préciser le degré de réalisme afin de conclure sur l'effet de réel et sur la problématique du local et de l'universel. En effet, les données temporelles disséminées dans ces récits ancrent précisément l'histoire dans une période déterminée ou déterminable et il est fait allusion à des événements du passé dont il est intéressant d'évaluer le réalisme.

S'il est apparent que Le Voile de Draupadi présente de nombreuses allusions historiques, il n'en est pas de même pour Rue la Poudrière et Le Sari vert où il n'est pas aussi simple de reconstituer exactement à quel moment se déroule le présent du récit. Néanmoins, malgré cette temporalité qui reste assez floue, les références historiques et le présent du récit des trois romans sont souvent traçables et vérifiables et contribuent à l'ancrage des romans dans une réalité plus ou moins locale. Les faits historiques mentionnés remontent parfois à un passé lointain. Ainsi, Rue la Poudrière brosse une île pré-indépendante où règne la misère, l'insalubrité et de nombreux maux sociaux qui affectent Paule, le personnage principal. Par contre, Le Voile de Draupadi décrit l'histoire de quatre générations de la famille d'Anjali et couvre une période qui remonte à la traversée des travailleurs engagés indiens. Dans Le Sari vert, des allersretours dans le passé ramènent à des événements très anciens qui se sont véritablement déroulés à l'île Maurice, et référence est même faite aux esclaves africains et malgaches à l'île Maurice.

Afin de bien mener notre étude, l'analyse de chaque roman suivra deux perspectives. La première sera de déterminer l'ancrage de l'histoire principale dans le temps, et la seconde sera de comparer les références historiques mentionnées aux événements réels qui ont eu lieu à l'île Maurice. Finalement, nous pourrons cerner dans quelle mesure ces romans sont réalistes et mauriciens.

De nombreuses études ont été consacrées à la traversée des coolies de l'océan Indien, aussi nommé Kala Pani ou "eau obscure"259. D'autres travaux se focalisent sur la condition difficile des travailleurs engagés, leur choc émotionnel après la séparation de leur pays d'origine et leur condition de vie une fois dans l'île, mais sans toutefois

\footnotetext{
${ }^{259}$ Rohini Bannerjee, «The Kala Pani Connection: Francophone Migration Narratives in the Caribbean Writing of Raphaël Confiant and the Mauritian Writing of Ananda Devi », Anthurium: a Caribbean Studies Journal, 7, 2009, p. 1-11; Véronique Bragard, «L'Empreinte des Kalapani dans la littérature caribéenne et mauricienne : une comparaison transcoloniale », L'Esprit créateur, 50, 2010, p. 86-94; Elizabeth Deloughrey, «On Kala Pani and Transoceanic Fluids », New Literature Review, 47, 2011, p. $71-92$.
} 
faire allusion aux trois romans que nous étudions. Il n'existe par ailleurs aucun travail axé sur l'aspect historique du roman Le Sari vert. Vu le peu de recherche faite à ce propos, la comparaison des références temporelles de l'histoire et des événements historiques mentionnés avec les interprétations des historiens ou les documents d'archives existants permettra de doser le réalisme historique des trois romans.

\section{Rue la Poudrière}

\section{L'ancrage de l'histoire}

Comme nous l'avons déjà vu, Françoise Lionnet souligne que le présent de l'histoire de Rue la Poudrière se déroule dans les années $1950^{260}$. Pour sa part, dans son introduction au roman, Vicram Ramharai reste plus vague et suggère, en ne donnant pas de date exacte, que le récit se serait déroulé à Port-Louis «avant les années $1980 »^{261}$, tandis que Devi le situe à la « fin des années soixante et dans la première moitié des années soixante-dix $»^{262}$ où :

Il y avait encore une misère intense, les gens étant pris dans le cercle vicieux de la pauvreté, de l'alcoolisme, de la prostitution. Il y avait des gens qui avaient faim, et qui vivaient dans un dénuement quasi total. Il y avait des 'îlois' qui dormaient sur le trottoir, des débardeurs qui s'étaient retrouvés du jour au lendemain sans travail. Il y avait des femmes qui subissaient toutes les violences et toutes les perversités sans avoir leur mot à dire, sans pouvoir espérer qu'elles seraient un jour entendues ${ }^{263}$.

Notre analyse approfondie du roman éclairera ces incertitudes concernant le présent du récit.

\footnotetext{
${ }^{260}$ «Until the 1950s, this street was frequented by prostitutes, and lined with "maisons closes" well known to visiting sailors, tourists and local bourgeois », Françoise Lionnet, "Evading the Subject: Narration and the City in Ananda Devi's Rue la Poudrière », L'Esprit créateur, Post-colonial women's writing, 33, 1993, p. 14.

${ }^{261}$ Ananda Devi, Rue la Poudrière, Abidjan, Nouvelles Editions Africaines, 1988, voir l'introduction du roman, sans pagination.

262 Ibid.

${ }^{263}$ Ibid., voir la préface du roman, sans pagination.
} 
Comme l'a remarqué Lionnet, le récit se déroule bien à partir des années 1950, à un moment où les Mauriciens vivaient dans la marginalité et le danger ${ }^{264}$, comme Paule, sa famille et ses amis, et où la construction de nouveaux bâtiments à Port-Louis a entraîné le déplacement des maisons closes dans les régions côtières ${ }^{265}$. Le roman fait également mention de deux incidents qui se sont vraiment déroulés à l'île Maurice dans les années 1979, notamment la grève des dockers et la démolition des anciens bâtiments de la Butte. Rue la Poudrière détaille la situation sociale déplorable de l'île Maurice des années 1950, 1960 et 1970.

\section{La grève des dockers de 1979}

Presqu'à la fin du roman, Tapsy, le client fidèle de Paule, révèle l'insatisfaction des travailleurs du port de Port-Louis: « les débardeurs font la grève [...] la nuit sera dure pour les dockers et les stevedores ${ }^{266}$. Il évoque aussi la raison de leur action et précise que c'est «à cause du "vrac" » ${ }^{267}$, terme qui désigne les produits transportés en vrac «sans emballage particulier ${ }^{268}$. Dans la réalité, les problèmes avaient commencé en juin 1971, et pour faire face aux conditions déplorables des travailleurs du port le Harbour Docks Workers Union avait initié la première grève des travailleurs le 21 juin $1971^{269}$. Cette grève a par la suite déclenché les nombreuses grèves que l'île Maurice a connues dans les années $1970^{270}$. Une des raisons qui a aussi poussé à cette grève de 1979 est, que les travailleurs de l'industrie revendiquaient « la reconnaissance de la SILU [Sugar Industry Labour Association] et de la l'UASI [Union of Artisans of the Sugar Industry], l'introduction de la semaine de 40 heures, 5 jours de travail pour les laboureurs [et] l'augmentation des salaires de 35 pour cent ${ }^{271}$. La grève dont fait

\footnotetext{
${ }^{264}$ Françoise Lionnet, «Evading the Subject: Narration and the City in Ananda Devi's Rue la Poudrière », L'Esprit créateur, Post-colonial women's writing, 33, 1993, p. 11.

${ }^{265}$ Ibid., p. 15.

${ }^{266}$ Ananda Devi, Rue la Poudrière, Abidjan, Nouvelles Editions Africaines, 1988, p. 147.

267 Ibid.

${ }^{268}$ D'après le rapport de la Truth and Justice Commission, "vrac" signifie en créole mettre en grand nombre sur une surface étendue, sans emballage ; "Kreol word meaning to lay out on a large surface without a wrapper », Truth \& Justice Commission, «Report of the Truth and Justice Commission. Volume 1 », p. 468, http://www.usip.org/sites/default/files/ROL/TJC_Vol1.pdf, consulté le 3 mars 2013.

${ }^{269}$ Selon le rapport de la Truth and Justice Commission, les travailleurs s'élevaient contre le refus d'une augmentation de salaire et revendiquaient une amélioration des conditions de travail, « The 1971 strike, more than any other event, was crucial for the future of harbour workers. Even if the strike did not start in the port itself, one of the employees' objectives was the readjustment of their pay [...] this strike was necessary and inevitable because employers were not in favour of demands made by workers », Ibid., p. 266.

${ }^{270}$ Lindsey Collen, « Another Side of Paradise », http://newint.org/features/2009/05/01/mauritius-class/, consulté le 18 septembre 2013.

${ }^{271}$ Le Defi Plus, « Histoire-1979 : les grévistes à deux doigts de la mort »,
} 
mention Tapsy est celle que les dockers ont entreprise en 1979 au Mauritius Sugar Bulk Terminal, où le sucre était stocké en vrac avant que les débardeurs l'emballent pour l'exportation. En effet, la construction du Mauritius Sugar Bulk Terminal a débuté en 1977 pour être achevée en $1980^{272}$, date à laquelle les docks ont été mécanisés pour pomper le sucre directement dans la cale des navires ${ }^{273}$. Avant que le Mauritius Sugar Syndicate ait pris la décision en 1971 de construire ce dock mécanisé, « quelque 2000 dockers [...] chargeaient 500000 tonnes de sucre dans des sacs de 50 à 80 kilos sur leurs dos $»^{274}$. La mécanisation du dock menaçait le travail des dockers qui mettaient quinze jours à embarquer tout le sucre nécessaire sur un navire, plutôt que trois jours avec la mécanisation du dock. D'après Philip English, pour la compagnie, cette mécanisation se faisait pressante car l'économie mauricienne du sucre était en plein essor de 1972-1979 suite à une forte croissance économique occasionnée par le commerce du sucre ${ }^{275}$. Cette grève de 1979 des travailleurs du dock qui a duré deux semaines ${ }^{276}$ et «qui avait donné [...] aux travailleurs une conscience de classe ${ }^{277}$, explique sans aucun doute pourquoi Tapsy remarque que «bientôt, ils ne pourront plus payer leur topette de rhum, leur drogue engourdissante, leur planche de salut $»^{278}$.

Ainsi, nous avons vu que cet événement, dont fait mention Tapsy et qui a eu lieu en 1979 à l'île Maurice, est décrit avec réalisme et révèle les problèmes de l'époque. Devi donne incontestablement beaucoup d'importance à l'espace et au cadre dans lequel évoluent les personnages, intégrant la couleur locale et créant un effet réel

http://www.defimedia.info/defi-plus/dp-societe/item/57259-histoire-1979-les-grevistes-a-deux-doigts-dela-mort.html, consulté le 3 août 2014.

272 The Mauritius Sugar Bulk Terminal Corporation, «The Mauritius Sugar Bulk Terminal Corporation », http://www.gov.mu/portal/sites/ncb/moa/mstc/background.htm, consulté le 3 septembre 2013.

273 «Come 1979, the docks were about to be mechanised, to pump sugar directly into the holds of ships », Lindsey Collen, «A Page in Revolutionary History. The 1979 General Strike in Mauritius », http://directaction.org.au/issue14/a_page_in_revolutionary_history_the_1979_general_strike_in_mauritius, consulté le 5 novembre 2012.

274 «Some 2000 dockers still loaded 500,000 tonnes of sugar in 50-80 kilogram bags on their backs », Ibid. 275 Philip English, «Mauritius Reigniting the Engines of Growth. A Teaching Case Study », http://siteresources.worldbank.org/WBI/Resources/wbi37136.pdf, consulté le 2 septembre 2013.

${ }^{276}$ Le gouvernement de l'époque refusait tout accord alors que les employeurs licenciaient ceux qui prenaient part aux grèves. C'est à ce moment que quelques activistes tels que Paul Bérenger et Ram Seegobin ont décidé de commencer une grève de la faim. C'est ainsi qu'après quatre jours "Lakor 23 UT" fut signé. Cet accord stipulait que «les usines ne fermeraient plus leurs portes et que tous les travailleurs qui avaient été licenciés pendant la grève seraient employés dans d'autres postes », Ram Seegobin, "Hunger Strikes: "Food Refusal" as a Means of Struggle », http://www.lalitmauritius.org/viewnews.php?id=965, consulté le 4 septembre 2013.

${ }^{277}$ Le Defi Plus, « Histoire-1979 : les grévistes à deux doigts de la mort », http://www.defimedia.info/defi-plus/dp-societe/item/57259-histoire-1979-les-grevistes-a-deux-doigts-dela-mort.html, consulté le 3 août 2014.

${ }^{278}$ Ananda Devi, Rue la Poudrière, Abidjan, Nouvelles Editions Africaines, 1988, p. 147. 
certain $^{279}$. A ce propos, Sainsbury affirme que la narration d'une histoire n'est en effet qu'un compte-rendu des vérités déjà connues que l'auteur relate de façon plus discrète $^{280}$. Exactement comme Devi fait part de la grève de 1979 sans donner de précision temporelle mais en mentionnant le "vrac" qui aide à déterminer le présent du récit. Aussi, nous pouvons conclure que Paule est née en 1959 et meurt en 1979 puisqu'au moment où elle meurt, la jeune fille est âgée de vingt ans ${ }^{281}$, et puisque la grève des dockers se passe en 1979.

\section{La destruction des bâtiments à la Butte}

Paule, qui est revenue à son ancienne demeure à la Butte peu de temps après la grève des dockers de 1979, a accidentellement ingurgité un poison et voit, dans son délire, «d'énormes monstres jaunes ${ }^{282}$ qui s'approchent du quartier pour détruire les bâtiments et faire place à des « façades de béton ou de ciment » ${ }^{283}$. Il n'est pas à exclure que cet épisode fasse allusion à la destruction réelle des vieux bâtiments de la Butte. C'est justement dans les années 1970 que le gouvernement mauricien avait mis en place de grands projets de restructuration au niveau de la santé, de l'éducation et du logement pour améliorer la qualité de vie de la population ; fait qui est confirmé dans le rapport du World Bank Group par l'économiste Ali Zafar ${ }^{284}$. Ce projet de reconstruction avait en effet été initié dans les années 1950 par l'architecte Thornton White, qui avait proposé un plan pour résoudre le problème de logement et améliorer la situation des dockers dans le nord et l'ouest de Port-Louis, justement là où se situe la Butte. Mais ce n'est que dans les années 1960 que les programmes du logement social pour les personnes à faibles revenus ${ }^{285}$ avaient débuté et que le Central Housing

\footnotetext{
279 «In such cases, the author provides "local color" by telling us things which not only are true, but which the reader is intended to realize he is supposed to treat as true », Mark Sainsbury, Fiction and Fictionalism. New Problems of Philosophy, London, Routledge, 2010, p. 4.

280 «Isn't a story-teller in some ways like someone who is recounting known truths? He will normally take care not to give rise to the impression he is really doing that », Ibid., p. 10.

281 «à vingt ans, qui pense à autre chose qu'une sortie le samedi soir », Ananda Devi, Rue la Poudrière, Abidjan, Nouvelles Editions Africaines, 1988, p. 115.

282 Ibid., p. 192.

283 Ibid.

${ }^{284}$ Ali Zafar, "Mauritius: an Economic Success Story", http://siteresources.worldbank.org/AFRICAEXT/Resources/Mauritius_success.pdf, $\quad$ consulté $\quad$ le 14 novembre 2013.

${ }^{285}$ «Social housing programmes for low income groups which had begun in the 1960s, gave way to a new housing strategy based on cost recovery through the setting up of a public company, The National Housing Development Company Limited (NHDC) », Office of the United Nations Resident Coordinator, Mauritius, « Common Country Assessment. Mauritius »,

http://www.law.yale.edu/rcw/rcw/jurisdictions/afe/mauritius/mauritius assessment.pdf, consulté le 3 septembre 2013, p. 45.
} 
Authority a été créé en 1970 pour lancer le programme de relogement ${ }^{286}$. Ainsi, 13000 maisons ont été construites vers 1970 dans les régions urbaines et rurales ${ }^{287}$ et à la Butte, quartier résidentiel pauvre situé dans l'ouest de Port-Louis qui avait souffert de glissements de terrains répétés ${ }^{288}$.

Tout compte fait, ces «énormes monstres jaunes » ${ }^{289}$, les bulldozers qui ont commencé en 1979 à démolir les anciens bâtiments en ruines de la Butte au moment où Paule meurt, correspondent au début de ce grand projet de relogement; ce qui vient confirmer que cet épisode du roman est basé sur un événement réel, mais qui est relativement peu connu. Dans une certaine mesure, Devi, à travers cet épisode, met la lumière sur la destruction des bâtiments de la Butte. Sainsbury affirme que ce genre littéraire éduque les lecteurs en insérant des faits réels qui ne sont autrement pas connus $^{290}$, comme le relogement à la Butte dans Rue la Poudrière qui est peu connu et encore moins mentionné. Il est tout aussi intéressant de noter que Devi a si ingénieusement incorporé et tissé la réalité à la fiction qu'il est difficile de séparer les deux, ce qui fait que le lecteur arrive difficilement à déchiffrer si la destruction de la Butte relève d'un événement fictif ou non, d'autant plus que Devi ne précise ni l'année ni la raison de ce relogement. C'est cette technique de représenter la réalité de façon subtile dont fait mention Maureen Ramsden lorsqu'elle énonce qu'une œuvre fictive doit contenir des informations sur la réalité de façon assez générale qui aideront les lecteurs à décoder le factuel du fictionnel ${ }^{291}$. Tout compte fait, dans cet épisode du relogement, Devi ne fait que mentionner l'endroit exact où ceci se passe mais cette piste nous a guidés pour résoudre l'énigme. Ceci est sans doute fait exprès par l'auteure

\footnotetext{
286 «The Central Housing Authority was set up and launched a programme for the re-housing », J. Addison, K. Hazareesing, A New History of Mauritius, Mauritius, Editions de l'océan Indien, 1989, p. 105.

287 « The houses were built all over the island in both urban and rural areas », Ibid.

288 «The La Butte area, a poor residential district located in the West of Port-Louis, the capital of Mauritius has suffered from repeated landslides », Port-Louis City Landslide Project, http://www.jica.go.jp/english/our_work/evaluation/oda_loan/post/2002/pdf/136_full.pdf, consulté le 3 septembre 2013.

${ }^{289}$ Ananda Devi, Rue la Poudrière, Abidjan, Nouvelles Editions Africaines, 1988, p. 192.

290 «The value of [...] fiction is precisely that it enables [...] to learn a (real world) truth which [...] otherwise would not have been known », Mark Sainsbury, Fiction and Fictionalism. New Problems of Philosophy, London, Routledge, 2010, p. 179.

291 «The acceptance by the reader of a particular way of transcending both factual and the fictional in written works involves the concept of verisimilitude. In order to enable the reader to decode a factual or fictional work in a particular genre, and belonging to a particular age, a work will usually exhibit clear generic signals », Maureen Ramsden, « Fictional Frontiers: the Interrelation of Fact and Fiction between the World and the Text », Neophilologus, 95, 2011, p. 347.
} 
qui joue avec l'intérêt du lecteur tout en ne donnant que peu d'informations afin de faire durer le suspense ${ }^{292}$.

\section{Un ancrage et un passé local et réaliste dans Rue la Poudrière}

En fin de compte, la référence à la grève du "vrac" dans le roman, permet de situer avec certitude l'histoire de Paule entre 1959 et 1979. La description réaliste de la grève des travailleurs du port de Port-Louis et celle de l'épisode de la démolition de la Butte font état de l'époque où les logements insalubres ont été remplacés entre 1960 et 1980 par des logements propres et modernes. Ces références à l'histoire locale de PortLouis ancrent de façon crédible l'histoire de Paule dans les années 1959 à 1979. Si d'une part, c'est à travers Tapsy que l'épisode de la grève est introduit, c'est Paule qui attire l'attention du lecteur sur la destruction des bâtiments de la Butte. De ce fait, agissant comme des agents moraux dans ce texte fictif ${ }^{293}$, Tapsy et Paule sont des «personnages-focalisateurs [...] qui attirent l'attention du lecteur sur un objet, un événement ou un autre personnage à travers leur propre regard $»^{294}$.

\section{Le Voile de Draupadi}

\section{L'ancrage de l'histoire}

Le Voile de Draupadi ne donne guère de référence temporelle exacte qui permette de situer l'histoire d'Anjali mais fait état de certains épisodes de l'histoire mauricienne, tels que la traversée des travailleurs engagés. A ce propos, Rohini Bannerjee commente les contraintes physiques et spirituelles ${ }^{295}$ de la traversée des travailleurs engagés, comme le grand-père d'Anjali. D'après elle, en quittant leur pays natal, les travailleurs engagés ont ressenti une grande perte physique et

\footnotetext{
292 «I like to play little games with the reader, leave some revelation to come at the end », Andre Borges, « Jaipur Literature Festival 2014: Music of Languages. An Interview with Ananda Devi », http://zeenews.india.com/entertainment/bookworm/jaipur-literature-festival-2014/music-of-languagesan-interview-with-ananda-devi_3165.htm, consulté le 13 juin 2014.

${ }^{293}$ «Within a fictional world the characters are responsible for their actions. They are moral agents », Frank Palmer, Literature and Moral Understanding. A Philosophical Essay on Ethics, Aesthetics, Education and Culture, Oxford, Oxford University Press, 1992, p. 110.

${ }^{294}$ Vincent Jouve, L'Effet-Personnage dans le roman, Paris, Presses Universitaires de France, 1992, p. 127-128.

295 «The fictional francophone works of both Caribbean writer Raphaël Confiant and Mauritian writer Ananda Devi recount the physical and spiritual dislocations of such Kala pani crossings », Rohini Bannerjee, «The Kala Pani Connection: Francophone Migration Narratives in the Caribbean Writing of Raphaël Confiant and the Mauritian Writing of Ananda Devi », Anthurium: a Caribbean Studies Journal, 7, 2009, p. 1.
} 
psychologique $^{296}$. Bannerjee ajoute que Devi se fie aux faits historiques et aux récits personnels des voyageurs pour aider les lecteurs à comprendre pourquoi tant d'Indiens, avec ou sans famille, ont quitté les provinces indiennes ${ }^{297}$. Pour elle, au lieu de nier cette période difficile de l'histoire mauricienne, le roman souligne à quel point cette rupture avec l'Inde est devenue une partie intégrante de l'identité historique mauricienne ${ }^{298}$. Nous nous intéresserons tour à tour aux épisodes historiques qui entrecoupent le récit, telle la traversée des travailleurs engagés et le sort des travailleurs engagés sur l'île.

Il est probable que le bateau dans lequel est venu le grand-père d'Anjali a accosté l'île entre 1920 et 1924 puisqu'il était venu dans l'un des derniers bateaux ${ }^{299}$. En effet, même si le roman n'indique ni le nom du bateau ni l'année précise de son arrivée, nous pouvons établir que le grand-père d'Anjali est arrivé entre 1920 et 1924, alors qu'il était un jeune homme estimé des autres ${ }^{300}$.

Par ailleurs, la date du 24 novembre 1986, inscrite à la fin du texte, peut être interprétée comme étant la date de la mort de Wynn, le fils d'Anjali. Puisque nous savons qu'Anjali avait 15 ans $^{301}$ à la mort de sa cousine qui remonte à 10 ans $^{302}$, nous pouvons estimer qu'Anjali est âgée de 25 ans en 1986. Autrement dit, elle serait née en 1961. Etant donné que la mère d'Anjali a 50 ans $^{303}$ en 1986, nous pouvons supposer qu'elle est née en 1936 et puisqu'elle s'est mariée à $17^{304}$ ans, son mariage remonterait à 1953. Cette hypothèse qu'Anjali est née en 1961 au moment où sa mère avait 25 ans,

\footnotetext{
296 « A great sense of loss and displacement, both physical and psychological, experienced by Indian indentured workers leaving their homeland [...]», Ibid., p. 2.

297 «Devi relies on historical fact and personal accounts of human experience and memory to help readers appreciate why so many Indians, with or without family, left the Indian provinces », Ibid.

298 «Instead of denying this difficult period of Mauritian history, Devi embraces it and underlines how breaking away from India to join an insular life on Mauritius has become an integral part of Mauritian historical identity », Ibid., p. 5.

299 « Mon grand-père, le père de l'oncle Sanjiva et de ma mère, était venu de l'Inde au début du siècle », Ananda Devi, Le Voile de Draupadi, Paris, L'Harmattan, 1993, p. 46.

300 «A l'avenir, l'île connaîtra la venue de plusieurs milliers de travailleurs indiens qui sont ainsi arrivés vers la fin de 1870 et jusqu'à la fin de 1924. Cette venue massive des Indiens explique pourquoi $68 \%$ de la population mauricienne comprend des Indo-Mauriciens dont $48 \%$ d'hindous », Office of Public Communication, Bureau of Public Affairs, «Historical, Political and Economic Overviews of the Countries of the World. Mauritius », http://dosfan.lib.uic.edu/ERC/bgnotes/af/mauritius9211.html, consulté le 23 octobre 2012.

301 «À quinze ans, j’avais été impressionnée, effrayée par Vasanti », Ananda Devi, Rue la Poudrière, Abidjan, Nouvelles Editions Africaines, 1988, p. 160.

302 «Maintenant, dix ans après sa mort », Ibid., p. 33.

303 « À cinquante ans, il ne reste plus en elle qu'un potentiel sans cesse grandissant de souffrance », Ibid., p. 63 .

${ }^{304}$ « À dix-sept ans, elle a fait un mariage convenable dans une famille de la même caste et du même clan », Ibid., p. 60.
} 
et que sa mère s'est mariée à 17 ans est fort probable vue qu'elle coincide avec le fait que les femmes hindoues de l'époque se mariaient jeunes et avaient des enfants jeunes.

\section{La traversée des jahaji bhai ${ }^{305}$}

Discutant de la traversée des travailleurs engagés comme le grand-père d'Anjali, l'historien Hugh Tinker note que ces Indiens venaient de tous les coins de l'Inde, notamment de Calcutta, Orissa, Bihar, Oudh, Madras ${ }^{306}$. Or, d'après Anjali, une fois sur le bateau, les travailleurs se liaient d'amitié et les passagers inconnus les uns des autres, au début du voyage, se créaient vite des liens de fraternité ; «s'appuyant les uns contre les autres, se forgeant des liens plus solides encore que ceux qui les avaient jadis liés à leurs familles et amis ${ }^{307}$. Tinker précise que même si ces voyageurs ne parlaient pas tous les mêmes dialectes, puisqu'originaires de régions différentes, une fois sur le bateau, le partage de la douleur physique et de la torture morale les rapprochaient à jamais.

Les coolies, tout comme les esclaves avant eux, avaient acquis la force, le sens de la camaraderie et même de la fraternité à travers les épreuves du voyage ${ }^{308}$ et $L e$ Voile de Draupadi fait ressortir ce fait : « les conditions du voyage étaient terribles » ${ }^{309}$. Fait souligné aussi par Tinker qui avance que ce voyage se résumait aux drames et à l'endurance ${ }^{310}$. Il explique aussi que ce sont les conditions climatiques et la taille des bateaux qui rendaient la traversée difficile et que ces voyageurs devaient faire face à toutes sortes de maladies et d'intempéries qui entrânaient souvent la mort. Si un voyage était fatigant mais tolérable, le suivant pouvait être un cauchemar inoubliable ${ }^{311}$. Les maladies aggravaient aussi la situation. Ainsi, lors du voyage du grand-père d'Anjali, une «dizaine de coolies [sont] morts en route ${ }^{312}$ et beaucoup étaient «moralement et physiquement épuisés par la dysenterie et la malnutrition » ${ }^{313}$. Authentifiant ce que raconte Anjali, Marina Carter relève la qualité douteuse des

\footnotetext{
${ }^{305}$ Ecrit en hindi, le terme "jahaji bhai” signifie les frères du bateau.

${ }^{306}$ Hugh Tinker, A New System of Slavery, London, The Camelot Press Ltd, 1974, p. 119.

307 Ananda Devi, Le Voile de Draupadi, Paris, L'Harmattan, 1993, p. 47.

308 «Certainly we may conclude that the coolies, like the slaves before them, acquired two gains from their voyage-fortitude, and a sense of comradeship and even brotherhood for those who had shared the passage with them », Hugh Tinker, A New System of Slavery, London, The Camelot Press Ltd, 1974, p. 118 .

${ }^{309}$ Ananda Devi, Le Voile de Draupadi, Paris, L'Harmattan, 1993, p. 46.

${ }^{310}$ Hugh Tinker, A New System of Slavery, London, The Camelot Press Ltd, 1974, p. 118.

311 «A great deal depended upon the conditions encountered by the different ships: one voyage would be tedious, but tolerable, the next would be a never-forgetting nightmare », Hugh Tinker, A New System of Slavery, London, The Camelot Press Ltd, 1974, p. 155.

${ }_{312}$ Ananda Devi, Le Voile de Draupadi, Paris, L'Harmattan, 1993, p. 47.

313 Ibid.
} 
aliments qui rendaient les voyageurs malades et les conditions déplorables dans les bateaux qui étaient si éprouvantes que certains n'hésitaient pas à mettre fin à leur vie en se jetant par-dessus bord ${ }^{314}$. Clare Anderson rejoint Carter et ajoute que la mortalité était causée par un manque d'approvisionnement ou la qualité douteuse de l'alimentation ${ }^{315}$. Anjali raconte de plus que les voyageurs étaient tous terrifiés, et que pour les aider à changer d'idées, ils avaient droit à des divertissements et amusements. Sans doute, les versets de la Gita récités par le grand-père faisaient partie de ces divertissements qui aidaient certainement à évacuer la peur associée à ce voyage long et périlleux. Tinker confirme que l'on encourageait les passagers à se divertir afin de diminuer leur angoisse et leur ennui ${ }^{316}$. Le Voile de Draupadi montre combien le voyage était difficile et irréversible :

Les conditions du voyage étaient terribles, mais plus effrayante encore était la conviction qu'ils ne pourraient jamais revenir en arrière, refaire le trajet à l'inverse. Ils avaient tous traversé le "Kala Pâni", l'eau noire de l'océan, et ils savaient qu'ils étaient déjà morts pour ceux de leur caste qui étaient demeurés en Inde, que le rituel des morts avait déjà été célébré en leur nom ${ }^{317}$.

Cette frayeur du non-retour du grand-père et des autres passagers n'était pas infondée car selon Tinker, les îles à l'étranger étaient généralement considérées par les Indiens comme Тари et tous ceux qui disparaissaient de leur village étaient considérés être partis à Tapu, un endroit maléfique ${ }^{318}$. En outre, pour Rohini Bannerjee, à part être considérés comme parias, les Indiens croyaient fermement que la traversée signifiait la

\footnotetext{
314 «His [a passenger named Ramasawmy, travelling from Karical, India to Mauritius aboard the Jacques Coeur in 1861] ill treatment on the ship had strengthened his resolve to take the risk of jumping into the sea », Marina Carter, Voices from Indenture. Experiences of Indian Migrants in the British Empire, London, Leicester University Press, 1996, p. 74.

315 "Although supplies were in theory inspected before ships departed, evidence suggests that captains sometimes skimped on them, and provisions either ran short or were of questionable quality », Clare Anderson, «Indian Convict Ship Mutinies in the Mid-Nineteenth Century », http://www.sasnet.lu.se/EASASpapers/22ClareAnderson.pdf, consulté le 11 septembre 2013.

316 «One rule stated: coolies must be encouraged to amuse themselves by harmless diversions and should be allowed to play on their drums, etc, till 8 bells. From time to time there were entertainments - dancing, singing, wrestling and single-stick play », Hugh Tinker, A New System of Slavery, London, The Camelot Press Ltd, 1974, p. 156.

317 Ananda Devi, Le Voile de Draupadi, Paris, L'Harmattan, 1993, p. 46- 47.

318 «Islands overseas were generally known as Тари: anyone who disappeared from the village (absconded, lost, or murdered) was said to have gone to Tapu, so there was an evil association with the whole business », Hugh Tinker, A New System of Slavery, London, The Camelot Press Ltd, 1974, p. 120.
} 
fin du cycle de réincarnation et l'impossibilité d'atteindre la caste supérieure ${ }^{319}$. Si le contrat stipulait que les travailleurs engagés pouvaient retourner en Inde, ceux qui l'ont fait, au lieu d'être accueillis à bras ouverts, étaient rejetés en tant que parias, impurs à tout jamais ${ }^{320}$. Ce qui implique que ceux qui entreprenaient le voyage vers la fin de la traite des travailleurs engagés, c'est-à-dire vers les années $1900^{321}$, comme le grandpère d'Anjali, savaient déjà qu'un retour au pays ne serait pas possible.

C'est ainsi qu'à travers son roman Devi fait une représentation très précise et frappante du voyage et des peines psychologiques subies par les passagers, représentation qui aide certainement les lecteurs à visualiser et comprendre le sort des coolies indiens. Indubitablement, les travaux de Hugh Tinker, Rohini Bannerjee, Marina Carter et Clare Anderson corroborent le fait que les conditions de la traversée de l'océan Indien vécues par le grand-père d'Anjali et les autres passagers reflètent la réalité. En empruntant les termes de Névine El Nossery, Devi a l'art de «former, façonner ou modeler l'expérience pour [...] représenter et non à proprement parler pour [...] inventer $\gg^{322}$ ce qui s'est passé sur ces bateaux. En plus, en présentant le grandpère d'Anjali comme étant le «guide ${ }^{323}$ et le «soutien ${ }^{324}$ des autres voyageurs, Devi incite les lecteurs de retrouver dans ce personnage « une image revalorisée ${ }^{325} \mathrm{du}$ coolie mauricien.

\section{Les travailleurs engagés une fois à l'île Maurice}

La traversée de l'océan a tellement marqué les travailleurs engagés indiens que même après s'être installés dans l'île, ils «entendaient de nouveau la houle inquiète, le clapotis des vagues contre la coque, ils sentaient le balancement du navire et leur mal

\footnotetext{
319 « By evading Mother India and the regeneration of waters of the Holy Ganges, many indentured Indians felt that crossing the forbidden Kala Pani meant the end of the reincarnation cycle and the possibility of never reaching higher caste status », Rohini Bannerjee, « The Kala Pani Connection: Francophone Migration Narratives in the Caribbean Writing of Raphaël Confiant and the Mauritian Writing of Ananda Devi », Anthurium: a Caribbean Studies Journal, 7, 2009, p. 3.

${ }^{320}$ «One young man went to Calcutta on the Ganges in October 1874, but [...] returned to Trinidad on the Foyle in November 1875. When he arrived at the ancestral village, he found that his father's name had been forgotten [...] he was told to be off for a pariah, and polluted for ever », Hugh Tinker, A New System of Slavery, London, The Camelot Press Ltd, 1974, p. 174.

321 «Between 1906 and 1924, around 1394 labourers were landed in Port-Louis harbour and in May 1924, the last batch of indentured workers set foot on Mauritian soil », Aapravasi Ghat, « Aapravasi Ghat Trust Fund Newsletter », 1, 2009, p. 11.

${ }^{322}$ Névine El Nossery, Témoignages fictionnels au féminin. Une réécriture des Blancs de la guerre civile algérienne, New York, Rodopi, 2012, p. 29.

${ }^{323}$ Ananda Devi, Le Voile de Draupadi, Paris, L'Harmattan, 1993, p. 48.

${ }^{324}$ Ibid.

${ }^{325}$ Vincent Jouve, L'Effet-Personnage dans le roman, Paris, Presses Universitaires de France, 1992, p. 62.
} 
de mer, l'odeur de fiel et d'urine dans la cale $»^{326}$ et qu'ils continuaient à chanter et prier pour calmer les esprits et chercher courage, comme l'observe Marina Carter. Selon elle, ces chants folkloriques des travailleurs engagés transformaient leur expérience en un acte d'héroïsme et de résistance ${ }^{327}$ et démontraient comment, convaincus par leurs recruteurs qu'ils trouveraient de l'or dans les rues de cette île, ils faisaient désormais face à la désillusion ${ }^{328}$. Si Devi présente les chants vocalisés des coolies comme une manière d'extérioriser les conditions difficiles du voyage, Carter estime plutôt que ce sont les conditions de vie dans les colonies qui ont donné lieu à ces chansons.

La précision avec laquelle la référence est faite aux chansons dans le roman et leur authentification par Carter relève du vraisemblable, qui est défini par Riffaterre comme étant cette idée qui a le pouvoir de remplacer la vérité tout en libérant la fiction des entraves des références et par El Nossery comme étant «la substitution de l'expérience réelle ou hypothétique par une structure rhétorique imagée, qui néanmoins semble véridique et que le lecteur tend à accepter $»^{329}$. Ainsi, Devi interprète les faits réels insistant sur les horreurs du voyage et ces épisodes semblent si réels pour le lecteur qu'il n'arrive pas à déchiffrer les faits réels du vraisemblable. Maureen Ramsden et Névine El Nossery notent que, si la réalité est filtrée par l'auteur et subit des distorsions lors de la phase d'interprétation ${ }^{330}$, « la fiction [peut] garantir à l'histoire le potentiel de ne pas s'effacer $»^{331}$. Dans ce cas, Devi semble privilégier le caractère symbolique de la traversée plus que le vraisemblable pour mieux suggérer l'horreur qu'ont subi les coolies indiens, aussi bien pendant le voyage qu'après. Ceci est d'autant plus plausible que le roman montre comment le grand-père d'Anjali et les autres coolies, une fois dans l'île, ont dû travailler dans les champs de canne dans des conditions rudes :

\footnotetext{
${ }^{326}$ Ananda Devi, Le Voile de Draupadi, Paris, L'Harmattan, 1993, p. 47.

327 « Once in the colonies, the folksongs of indentured labourers turn the depiction of migration into a heroic act of resistance », Marina Carter, Voices from Indenture. Experiences of Indian Migrants in the British Empire, London, Leicester University Press, 1996, p. 80.

328 «The following song from Mauritius, by contrast, acknowledges the exaggerated hopes of emigrants convinced by recruiters that they would find gold on the streets of that island and stresses the reality of disappointment », Ibid.

${ }^{329}$ Névine El Nossery, Témoignages fictionnels au féminin. Une réécriture des blancs de la guerre civile algérienne, New York, Rodopi, 2012, p. 69.

330 «In written discourse, as also in our perception of the world, reality - which is necessarily filtered through the writer's own personal viewpoint, is subject to distortions both at the level of interpretation and of presentation. Writers are also often led to include the subjective, the imaginative and the anecdotal », Maureen Ramsden, «Fictional Frontiers: the Interrelation of Fact and Fiction between the World and the Text », Neophilologus, 95, 2011, p. 347.

${ }^{331}$ Névine El Nossery, Témoignages fictionnels au féminin. Une réécriture des blancs de la guerre civile algérienne, New York, Rodopi, 2012, p. 200.
} 
Ils traversèrent ensemble nombreuses épreuves et des rites de passage. Ils en acquirent les mêmes rides et les mêmes regards, plus étroitement unis encore, soudés par leur expérience commune comme des enfants unis par la $\operatorname{peur}^{332}$.

Toutefois, même si le grand-père d'Anjali travaille dur sur les propriétés sucrières, il décide de protester car il s'oppose au traitement que les travailleurs subissent. Anjali raconte que son grand-père, prêt à tous les sacrifices, « fit plusieurs grèves de la faim pour obtenir une amélioration de leurs conditions ${ }^{333}$. Il a même voulu se soumettre au pire en menaçant « de se brûler vif » ${ }^{334}$. Ce fait, confirmé par la Truth and Justice Commission ${ }^{335}$ est aussi discuté par Carter qui avance qu'en vérité, dans les propriétés sucrières, les travailleurs engagés pouvaient poser une plainte formelle auprès du magistrat local ou $\mathrm{du}$ «Protector of Immigrants » ${ }^{336}$ mais qu'en général, ils craignaient des représailles de la part des colons anglais et de ce fait, ils n'avaient pas d'autres choix que la grève de la faim pour se faire entendre. Ainsi, la grève de la faim du grand-père d'Anjali est plausible. Par ailleurs, Carter estime que la plupart des plaintes des travailleurs engagés étaient liées au châtiment physique et à l'humiliation ${ }^{337}$. Les laboureurs exprimaient souvent leur insatisfaction concernant leurs logements, nourriture et traitements médicaux ${ }^{338}$; fait aussi mentionné dans le roman où les coolies à la fin du voyage sont «tous moralement et physiquement épuisés par la dysentrie et la malnutrition ${ }^{339}$.

\footnotetext{
${ }^{332}$ Ananda Devi, Le Voile de Draupadi, Paris, L'Harmattan, 1993, p. 47.

${ }^{333}$ Ibid., p. 48.

334 Ibid.

335 «Living and Working Conditions on Sugar Estates », Truth \& Justice Commission, « Report of the Truth and Justice Commission. Volume $1 »$, http://www.usip.org/sites/default/files/ROL/TJC_Vol1.pdf, consulté le 3 mars 2013.

${ }^{336}$ Marina Carter, Voices from Indenture. Experiences of Indian Migrants in the British Empire, London, Leicester University Press, 1996, p. 106.

${ }^{337}$ « The majority of complaints articulated by indentured Indians dealt with the humiliating and painful physical chastisement they had suffered, and with the failure of employers to meet specific contractual obligations », Ibid.

338 «Labourers often expressed dissatisfaction with their lodgings, rations and medical treatment. Over tasking and insufficient pay - usually the result of excessive deductions - were common causes of complaint », Ibid.

339 Ananda Devi, Le Voile de Draupadi, Paris, L'Harmattan, 1993, p. 47.
} 


\section{Un ancrage local et réaliste et un passé à la fois local et universel dans Le Voile de Draupadi}

Il ressort que Le Voile de Draupadi offre une description véridique des conditions rudes de la traversée et de la souffrance morale et physique des coolies par l'entremise du grand-père d'Anjali même si l'année exacte et le nom du bateau à bord duquel le grand-père d'Anjali était venu sont inconnus. Ce grand-père s'affirme en tant qu'un leader et proteste contre les abus qui ont lieu dans les établissements sucriers comme l'ont fait certains coolies dans la réalité, ainsi que l'attestent les travaux de Hugh Tinker, Rohini Bannerjee, Marina Carter, Clare Anderson aussi bien que les faits révélés dans le rapport de la Truth and Justice Commission. C'est ainsi qu'à travers les personnages fictifs et un cadre réel Devi démontre une facette réelle du passé douloureux que l'île Maurice a connu.

\section{Le Sari vert}

Les indications temporelles dans Le Sari vert sont peu nombreuses et souvent problématiques car le récit présente des allers-retours incessants entre le passé et le présent du récit. Nous allons d'abord essayer de situer l'ancrage historique de l'histoire, avant d'examiner les événements historiques mentionnés dans le roman : l'épisode de la falaise du Morne, les émeutes de 1937 dans les établissements sucriers à Camp Kenya, la marche du sucre et le conflit racial de janvier 1968.

\section{L'ancrage de l'histoire}

Une lecture minutieuse du Sari vert révèle que Kitty est âgée de soixante$\operatorname{deux}^{340}$ ou soixante-quatre ans ${ }^{341}$ et que Malika, la fille de Kitty est, elle, âgée d'une quarantaine d'années ${ }^{342}$ au moment où le protagoniste est en train de mourir d'un cancer de la prostate.

S'il n'existe aucune indication directe qui donne l'âge du vieil homme, nous pouvons déduire du roman qu'il était déjà médecin pratiquant en 1922. En effet, le récit indique qu'il était en route vers Camp Kenya pour soigner un blessé pendant les

\footnotetext{
${ }^{340}$ «Quant à l'autre [Kitty], elle doit avoir soixante-deux, soixante-trois », Ananda Devi, Le Sari vert, Paris, Gallimard, 2009, p. 11.

341 «Une vieille de soixante-quatre ans », Ibid., p. 16.

342 «Enfin, la jeune [Malika], elle a ses quarante ans passés », Ibid., p. 11.
} 
«émeutes et grèves dans les propriétés sucrières » ${ }^{343}$ que la Commission d'enquête de Hooper situe en $1937^{344}$.

De plus, dans cet épisode, le chauffeur de taxi et le médecin se remémorent le moment, quinze ans auparavant ${ }^{345}$, où le médecin avait refusé de soigner la mère du chauffeur, très malade ${ }^{346}$ et où il avait donné une gifle à Kitty ${ }^{347}$ alors que celle-ci avait un peu plus de deux ans ${ }^{348}$. Ce qui semble confirmer le fait que Kitty parle mal ${ }^{349}$ et met encore les doigts dans la bouche ${ }^{350}$ lors de cet épisode. Ce qui est également confirmé par le fait qu'à la naissance de Kitty vers 1920 , sa mère a dix-sept ans ${ }^{351}$. En plus, il est indiqué que la femme du médecin est morte à vingt ans ${ }^{352}$ alors que sa fille Kitty avait deux ans ${ }^{353}$. Tout ceci suggère que Kitty est née juste avant 1920.

Si Kitty a entre soixante-deux et soixante-quatre ans quand son père est mourant, le présent du récit se situe dans la première moitié des années 1980, ce que semble confirmer le fait qu'il est question dans le roman d'ordinateurs ${ }^{354}$, appareils qui ne sont apparus dans les maisons que vers $1985^{355}$.

Par ailleurs, si Kitty avait deux ans quand sa mère est morte à l'âge de vingt ans $^{356}$, il en découle que la mère de Kitty serait née vers 1900 . Or, le roman indique

\footnotetext{
${ }^{343}$ Ibid., p. 83.

344 « The first news of any concerted action of importance indicative of discontent on the part of workers on sugar estates came from the Flacq disctrict. This was on the $30^{\text {th }}$ July, 1937 », Charles Hooper, Report of the Commission of Enquiry into Unrest on Sugar Estates in Mauritius, 1937, Port-Louis, Government Printer, 1938, p. 9.

345 «C'était il y a quinze ans », Ananda Devi, Le Sari vert, Paris, Gallimard, 2009, p. 86.

346 « Ma mère [la màre du chauffeur] était très malade, elle avait mangé quelque chose qui l'avait empoisonnée », Ibid.

347 «Une fois, elle [Kitty] s'est endormie ainsi, assise sur une chaise raide, un verre de lait entre les mains. Le lait s'est renversé sur elle et par terre. Elle n'a rien dit [...] Ce regard bovin m'a [le médecin] tellement énervé que la gifle est partie, si fort que Kitty a basculé comme un mannequin de bois »; «Je reconnais que c'était totalement dérisoire, à la limite de l'absurdité ; je reconnais l'insulte et le camouflet impossible à oublier, même après quinze ans », Ibid. p. 37-38, 87.

348 «lle [Kitty] avait [...] deux [ans] quand sa mère est morte », Ibid., p. 68.

349 «Non, tu vas la mourir, dit-elle », Ibid., p. 106.

350 « Kitty a mis les deux doigts dans sa bouche », Ibid., p. 106.

351 «Elle avait dix-sept ans et elle n'avait pas compris que la vie de couple n'était pas une plaisanterie »; "Quand on est femme, quand on s'apprête à être mère, on ne peut rire de la même façon »; "Elle venait d'avoir dix-sept ans », Ibid., p. 25, 26, 56.

352 «La femme que j'ai connue à quinze ans, que j'ai tuée à vingt ans », Ibid., p. 210.

353 «Elle avait [deux ans] quand sa mère est morte », Ibid., p. 68.

354 « Pas vous l'obscène derrière son écran d'ordinateur », Ibid., p. 184.

${ }^{355}$ Kelvin Soh, «TIMELINE: Key dates in the history of the personal computer », http://www.reuters.com/article/2009/01/07/us-laptop-sb-idUSTRE50601V20090107, consulté le 15 novembre 2013 .

356 « [...] j'ai souffert pour la morte même si vous ne le croyez pas, ces dernières images d'elle, la plaie ouverte de ses yeux saignants, pauvre femme de vingt ans qui n'avait rien compris à la vie, ces derniers instants où elle a cru pouvoir tromper ma vigilence [...]», Ibid., p. 191.
} 
qu'en 1968 la femme du médecin était toujours vivante ${ }^{357}$. De ce fait, il existe un anachronisme d'environ quarante-huit ans.

\title{
La falaise du Morne, les esclaves africains et malgaches à l'île Maurice
}

Le roman fait allusion aux esclaves par l'intermédiaire de Kitty qui, parlant à son père de la peur ${ }^{358}$ qu'elle avait de lui lorsqu'elle était enfant, lui raconte qu'elle est enfin prête à se libérer de son emprise, tout comme les esclaves malgaches et africains qui avaient choisi, malgré l'opposition de leur maître, la voie de la liberté en se jetant de la falaise du Morne :

\begin{abstract}
C'est comme les esclaves marrons qui ont fui jusqu'au Morne [...] ayant eu sur les lèvres et dans le sang le goût de la liberté [...] ils ont couru ensemble vers le bord de la falaise et ils se sont envolés [...] prenant le vent et suivant le soleil, vers les côtes de l'Afrique qu'ils n'espéraient plus jamais revoir ${ }^{359}$.
\end{abstract}

D'après le site du Morne Heritage Trust Fund, il y a en effet eu des esclaves malgaches et africains à l'île Maurice entre 1638-1710, et ce phénomène s'est répandu en $1721^{360}$. Selon des témoignages et les légendes orales, des esclaves rebelles auraient grimpé les terrains difficiles de la montagne du Morne, village situé à l'extrême sudouest de l'île, et auraient converti ses anfractuosités en un abri permanent ${ }^{361}$. Une autre version serait qu'après l'abolition de l'esclavage un escadron aurait grimpé la montagne pour annoncer l'excellente nouvelle aux fugitifs, qui pris par surprise, se seraient jetés de la montagne ${ }^{362}$, par peur d'être rattrapés et enchaînés de nouveau. Un

\footnotetext{
357 « La première étonnée de ce déferlement d'adoration a été, bien entendu, ma chère épouse », Ibid., p. 131 .

${ }_{358}^{2}$ La peur est débilitante au point où rien d'autre ne compte, ni envies, ni espoirs, ni possibilité de rébellion. La peur est l'esclave ultime, d'où on ne sort que par la mort », Ananda Devi, Le Sari vert, Paris, Gallimard, 2009, p. 198-199.

${ }^{359}$ Ibid., p. 199.

${ }^{360}$ Le Morne Heritage Trust Fund, « Slavery and Marooning », http://www.gov.mu/portal/sites/lemorne/history.htm\#MaroonsandLeMorne, consulté le 15 novembre 2013.

361 «These rebellious slaves ascended the tricky terrain and steep rock from formations of this mountain, converted its crevices into permanent shelter and depended on its thick vegetation to avoid detection and evade capture by parties who might seek to apprehend them », Candice Lowe, Indrani Gopauloo, Colette Le Chantier, Diana Bablee, Le Morne Cultural Landscape. History, Symbolism and Tradition, PortLouis, Mauritius, Government Printing Department, 2010, p. 23.

362 «It is said that after the abolition of slavery in 1835 , a squadron climbed the mountain to announce the wonderful news to the fugitives who had taken refuge there. It appears that soldiers succeeded in forging their way towards the maroons. It was then dramatic! Taken by surprise and thinking that the
} 
troisième compte-rendu oral de cet incident suggère que les esclaves du Morne se seraient jetés au bas de la falaise afin d'éviter d'être capturés ${ }^{363}$ par leurs maîtres et ramenés aux champs.

C'est cette dernière version que Kitty relate puisqu'elle dit à son père que les esclaves «ont été pourchassés, acculés au bord extrême du rocher » ${ }^{364}$. Kitty rapporte les souffrances et l'impuissance de ces esclaves qui « ont couru ensemble vers le bord de la falaise $»^{365}$ en espérant traverser les eaux instables de l'océan Indien en quête de leur patrie perdue ${ }^{366}$. De ce fait, cette falaise agit non pas comme un obstacle mais comme un facilitateur qui les aide à réaliser leurs rêves ${ }^{367}$, tout comme Kitty qui parvient à se soulager auprès de son père du fardeau de son passé lourd de secrets qui la hantent.

\section{Les émeutes de juillet 1937 dans les établissements sucriers à l'île Maurice}

Le docteur Bissam se souvient de la période trouble des émeutes lorsqu'il était jeune médecin à l'île Maurice. Il se rappelle très bien qu'un soir un homme lui avait demandé «en pleines émeutes et grèves dans les propriétés sucrières » ${ }^{368}$ d'aller soigner son frère gravement blessé, un des «laboureurs en grève contre les propriétaires des usines sucrières ${ }^{369}$. Evoquant la cause de ces émeutes, le médecin rapporte que « les propriétaires des usines broyaient les journaliers et ruinaient d'un seul coup les petits planteurs en décrétant que leurs cannes n'étaient plus conformes ${ }^{370}$. L'île Maurice a, réellement, été témoin de grèves sanglantes du 30

soldiers were lost, the maroons threw themselves from the mountain and were inevitably crushed to pieces at the foot of the cliffs », Ibid., p. 71.

363 «Depending on the version, maroons' rejection of servitude and their pursuit of freedom was insinuated up to three times: first, as the escape of slavery to hide/reside on the mountain; second in the news of abolition of slavery, carried by British soldiers; and third in the choice of maroons to hurl themselves off the cliffs of Le Morne - an act intended to avoid recapture and which represented the commitment of maroons to the preservation of life », Ibid., p. 76.

${ }^{364}$ Ananda Devi, Le Sari vert, Paris, Gallimard, 2009, p. 199.

365 Ibid.

${ }^{366}$ «The Passe des Marrons, today known as Passe de l'Ambulante, at the foot of the mountain, provided an escape route for runaway slaves who dares attempt to reverse the middle passage and cross the unstable waters of the Indian Ocean in search of lost homelands », Candice Lowe, Indrani Gopauloo, Colette Le Chantier, Diana Bablee, Le Morne Cultural Landscape. History, Symbolism and Tradition, Port-Louis, Mauritius, Government Printing Department, 2010, p. 23.

367 « The continued or successive occupation of Le Morne Brabant mountain by maroons and its perception as a desired destination prior to the abolition of slavery, testifies to the view that, in contrast to colonists, slaves saw Le Morne not as an obstacle but as a facilitator for the actualization of their dreams », Ibid., p. 71.

368 Ananda Devi, Le Sari vert, Paris, Gallimard, 2009, p. 83.

${ }^{369}$ Ibid., p. 40.

${ }^{370}$ Ibid., p. 84. 
juillet au 17 août $1937^{371}$ et une des raisons de cette grève, d'après l'historien Storey Kelleher, était liée au fait que les petits planteurs utilisaient une variété spécifique de canne à sucre appelée "Uba" pour répondre aux énormes besoins en sucre, que les usines n'appréciaient pas parce qu'elle était trop fibreuse ${ }^{372}$. Comme le dit le docteur Bissam, la commission d'enquête de Charles Hooper confirme que la rébellion avait commencé dans l'usine sucrière de Sans Souci, dans le district de Flacq, dans l'est de l'île, parce que les planteurs avaient vu une notice qui les avertissait qu'ils seraient payés 15 pour cent de moins pour la variété «Uba $»^{373}$.

Le médecin remarque aussi que les coupeurs de canne et leurs familles «mouraient de faim ${ }^{374}$. L'enquête de Charles Hooper établit que la plainte principale des journaliers en grève était que leurs salaires étaient trop bas ${ }^{375}$. Selon cette Commission d'enquête de 1938, si les coupeurs de canne et leurs familles recevaient de l'argent et de la nourriture, la qualité de la ration était mauvaise ${ }^{376}$, et les maîtres la supprimaient pour ceux qui étaient absents du travail, même pour cause de maladie ${ }^{377}$.

Le docteur Bissam ajoute que les petits planteurs «étaient habitués à vivre à genoux ${ }^{378}$ et avaient l'habitude «de toujours dire oui mo bourzois, bien mo bourzois ${ }^{379}$. Kelleher explique la soumission des petits planteurs par le fait qu'ils n'avaient pas accès aux journaux, à cause de l'analphabétisme ${ }^{380}$. Leur soumission étant considérée comme normale, les propriétaires n'auraient jamais cru que ceux-ci auraient un jour le courage de protester. C'est pourtant ce qu'ils ont fait, comme Bissam l'a remarqué : les coupeurs de canne «ont déposé leurs serpes. Ils ont refusé de

\footnotetext{
371 «Sequence of events during the period of unrest. Strikes and police measures. $30^{\text {th }}$ July $-17^{\text {th }}$ August », Charles Hooper, Report of the Commission of Enquiry into Unrest on Sugar Estates in Mauritius, 1937, Port-Louis, Government Printer, 1938, p. vii.

372 «In 1937, the worst outbreak of violence in the island's history began as a dispute between factories and small planters over a particular sugar cane variety called "Uba" [...]. The small planters found this cane ideally suited to their needs in the highly competitive sugar economy, while the factories disliked its high fibre content intensely », Kelleher Storey, « Small-Scale Sugar Cane Farmers and Biotechnology in Mauritius: the "Uba" Riots of 1937 », Agricultural History, 69, 1995, p. 166-167.

${ }^{373}$ Charles Hooper, Report of the Commission of Enquiry into Unrest on Sugar Estates in Mauritius, 1937, Port-Louis, Government Printer, 1938, p. 53.

${ }^{374}$ Ananda Devi, Le Sari vert, Paris, Gallimard, 2009, p. 84.

375 «The principal complaint of the casual labourer is that his wages are too low », Charles Hooper, Report of the Commission of Enquiry into Unrest on Sugar Estates in Mauritius, 1937, Port-Louis, Government Printer, 1938, p 165.

376 « The most usual complaint is that the quality of the rations is bad », Ibid., p. 163.

377 Ibid.

${ }^{378}$ Ananda Devi, Le Sari vert, Paris, Gallimard, 2009, p. 84.

${ }^{379}$ Ibid.

380 «Small planters lacked access to the newspapers and other public forums of debate because of widespread discrimination and illiteracy », Storey Kelleher, "Small-Scale Sugar Cane Farmers and Biotechnology in Mauritius: the "Uba” Riots of 1937 », Agricultural History, 69, 1995, p. 167.
} 
couper la canne ${ }^{381}$. Le docteur ajoute que les maîtres des établissements ont riposté en envoyant « des hommes pour tabasser les laboureurs et les obliger à reprendre la coupe, sur les genoux s'il le fallait ${ }^{382}$. Ce fait mentionné dans le roman est aussi confirmée dans la commission d'enquête de Hooper qui décrit cet épisode en soulignant que la baisse de 15 pour cent des salaires avait abouti à une cessation totale du travail ${ }^{383}$ suite à la décision des petits planteurs qui avaient l'intention de boycotter les établissements sucriers $^{384}$. En représailles, les propriétaires avaient eu recours à des intimidations ${ }^{385}$, comme en ont témoigné les travailleurs disant avoir été battus ${ }^{386}$; fait confirmé par Hooper $^{387}$.

Dans le roman, le docteur Bissam qui allait soigner ces travailleurs rebelles, a été témoin des dégâts occasionnés lors ces émeutes: «des champs brûlés, des corbeilles renversées, des charrettes cassées » ${ }^{388}$, une «vache mourante ${ }^{389}$ dont « les quatre pattes [étaient] sectionnées avec une netteté chirurgicale ${ }^{390}$. Hooper atteste que les émeutiers de 1937, en colère, avaient renversé une douzaine de camions chargés de sucre $^{391}$ et qu'ils avaient jeté des pierres ${ }^{392}$. Cette grève avait aussi fait quatre morts et six blessés ${ }^{393}$. Aussi, le docteur Bissam avait dû sauver la vie d'un homme, habitant Camp Kenya, car « une balle lui a fait éclater le genou et une autre lui a traversé la cuisse ${ }^{394}$. Ces faits révélés par le médecin rejoignent ceux de la commission d'enquête qui affirme que les tirs sur la foule avaient pris une tournure mortelle ${ }^{395}$.

\footnotetext{
${ }^{381}$ Ananda Devi, Le Sari vert, Paris, Gallimard, 2009, p. 84.

${ }^{382}$ Ibid.

383 " It was not until $30^{\text {th }}$ July that the dissatisfaction at the cut manifested itself in the form of a cessation of work », Charles Hooper, Report of the Commission of Enquiry into Unrest on Sugar Estates in Mauritius, 1937, Port-Louis, Government Printer, 1938, p. 10

384 «The small planters intended to boycott the estate », Ibid.

385 «There were allegations of intimidation », Ibid., p. 11.

386 «We have been beaten », Ibid.

387 «We have therefore come to the conclusion that cases of interference with workers did occur », Ibid., p. 54.

${ }_{388}$ Ananda Devi, Le Sari vert, Paris, Gallimard, 2009, p. 107.

${ }^{389}$ Ibid., p. 41

${ }^{390}$ Ibid.

391 « They had already overturned about a dozen trucks with sugar canes », Charles Hooper, Report of the Commission of Enquiry into Unrest on Sugar Estates in Mauritius, 1937, Port-Louis, Government Printer, 1938, p. 21.

392 «For the first time violence was resorted to by the crowd at Mon Désert in the shape of throwing stones and similar objects », Ibid.

393 « The total number of casualties inflicted by the firing were four dead, of whom two died in hospital, and six wounded », Ibid., p. 29.

${ }^{394}$ Ananda Devi, Le Sari vert, Paris, Gallimard, 2009, p. 108.

395 « Other shots, however, were fired which took deadly effect on the mob », Charles Hooper, Report of the Commission of Enquiry into Unrest on Sugar Estates in Mauritius, 1937, Port-Louis, Government Printer, 1938, p. 28.
} 
Si la commission d'enquête a pour sa part montré que les incidents avaient débuté dans la propriété de Riche Fond, ou Rich Fund, dans le district de Flacq, le 30 juillet $1937^{396}$, elle fait aussi état de dégâts produits un peu partout dans l'île, que ce soit dans l'est ou le $\operatorname{sud}^{397}$. Le roman décrit des incidents qui se sont déroulés dans la propriété sucrière de Mon Trésor Mon Désert et se concentre sur un blessé au Camp Kenya, endroit qui se situe non loin de Mon Trésor Mon Désert, toujours dans le district de Mahébourg. Ces événements que raconte le docteur dans le roman reflètent parfaitement des incidents réels de 1937. Il est frappant de constater le degré de réalisme de cet épisode du Sari vert. Les événements de la commission d'enquête de Hooper et ceux mentionnés dans le roman montrent à quel point « la frontière est mince entre fiction et réalité ${ }^{398}$. Ainsi, même si Le Sari vert ne contient pas de personnages historiques, les événements mentionnés relèvent de faits historiques de l'île Maurice qui sont vérifiables.

Ce tissage entre réalité et fiction permet, d'après Ramsden, d'intégrer des éléments réels dans un roman social pour aider les lecteurs à se situer dans cet encadrement temporel précis ${ }^{399}$. D'après El Nossery, ce genre de roman relève du témoignage fictionnel qui « est un genre où la réalité et la fiction sont intrinsèquement imbriquées, à tel point qu'il est souvent difficile de distinguer les frontières qui les séparent ${ }^{400}$. Ceci dit, même s'il est impossible d'authentifier l'épisode de la vache mourante ou encore l'histoire du planteur blessé au genou par une balle, ces éléments rendent encore plus vraisemblable ce texte fictif.

\footnotetext{
${ }^{396}$ « The first strike to break out, with exception of the small strike at Chébel Estate, was that which occurred at Rich Fund in the Flacq district on the $30^{\text {th }}$ July $1937 »$, Ibid., p. 53.

397 «It was therefore with considerable alarm that we heard from the Commissioner of Police that estate authorities in the South were likewise arming themselves with a view to defence in the event of an attack upon their lives or property », Ibid., 55.

${ }^{398}$ Vincent Jouve, L'Effet-Personnage dans le roman, Paris, Presses Universitaires de France, 1992, p. 199.

${ }^{399}$ « The inclusion of references to real people and places is of particular importance in an historical or social novel. It would seem to necessitate a retaining of factual status of these elemnts from the real world which situate the reader in a particular time and space and create expectations in relation to the factual elements with which the writer of fiction must work », Maureen Ramsden, « Fictional Frontiers: the Interrelation of Fact and Fiction between the World and the Text », Neophilologus, 95, 2011, p. 349350 .

${ }^{400}$ Névine El Nossery, Témoignages fictionnels au féminin. Une réécriture des Blancs de la guerre civile algérienne, New York, Rodopi, 2012, p. 151.
} 


\section{La marche du sucre}

Le vieux médecin rapporte que lors d'une cérémonie de mariage, accompagné de sa jeune femme, il parlait de construire «l'avenir d'un pays pas encore indépendant ${ }^{401}$. Ceci indique clairement qu'à ce moment, l'île est toujours colonisée par les Anglais ${ }^{402}$. En fait, le jeune docteur rêve de les expulser de Maurice tout comme Nehru et Gandhi les avaient chassés d'Inde : «nous chasserions les Anglais de notre île, nous serions les pionniers, nous marcherions sur les traces de Nehru et de Gandhi ${ }^{403}$. Le jeune docteur et ses amis pensent que les Mauriciens auront leur « route du sucre ${ }^{404}$ comme la « route du sel ${ }^{405}$ de Gandhi : «nous aurions notre route du sucre pour faire écho à leur route du sel» ${ }^{406}$. Celle de Gandhi avait été entreprise par Gandhi le 12 mars $1930^{407}$ pour protester pacifiquement contre les taxes et le monopole de la production du sel imposés par les Anglais. Le médecin et ses amis établissent expressément une comparaison entre ce qui s'était produit en Inde et ce qui allait se produire à l'île Maurice et aspirent à l'indépendance. Cette référence par le médecin, à la "route du sel" confirme le fait que cette conversation entre le docteur et les autres présents au mariage se déroulait avant mars 1968, avant la naissance de Kitty et avant l'indépendance de l'île Maurice.

\section{Le conflit racial de janvier 1968 à l'île Maurice}

Le vieux médecin se souvient aussi des émeutes auxquelles il a été mêlé juste avant l'indépendance : «ce pays n'avait même pas commencé à exister que, déjà, il se désintégrait $»^{408}$. Il se rappelle très bien les incidents qui s'étaient produits lorsqu'il travaillait à l'hôpital civil, le premier hôpital de l'île Maurice ${ }^{409}:$ « une nuit je rentrais

\footnotetext{
401 Ananda Devi, Le Sari vert, Paris, Gallimard, 2009, p. 26.

402 Ceci est une autre indication qui démontre que le médecin avait commencé à travailler avant mars 1968, la date où l'île a obtenu son indépendance.

${ }^{403}$ Ananda Devi, Le Sari vert, Paris, Gallimard, 2009, p. 26.

${ }^{404}$ Ibid.

405 Ibid.

${ }^{406}$ Ibid.

${ }^{407}$ Scott Graham, « Gandhi’s Salt March to Dandi », http://postcolonialstudies.emory.edu/ghandis-salt-march-to-dandi/, consulté le 17 septembre 2013. ${ }_{408}$ Ananda Devi, Le Sari vert, Paris, Gallimard, 2009, p. 128.

409 «The first 40-bed hospital of the capital built in 1733 under governor de Maupin at [present day] Edith Cavell Street was destroyed by a cyclone. In January 1740 governor Labourdonnais opened the first wing of a new 240-bed hospital called Civil Hospital at the seaport while the second wing completed in June 1740 took the name of Military Hospital [...] In November 1984, the Civil Hospital was renamed Dr Abdool Gaffoor Jeetoo Hospital after a well-known pre-independent political leader who died in January 1961 », Ministry of Health and Quality of Life, « Dr. A. Gaffoor. Jeetoo Hospital », http://www.gov.mu/portal/site/mohsite/menuitem.4581ded5607bad351a42860aa0208a0c/, consulté le 12 mai 2014.
} 
chez moi, épuisé et inquiet à cause des désordres et des émeutes qui avaient éclaté dans l'île ${ }^{410}$. Tout comme pour le docteur Bissam, ces incidents sont restés gravés dans la mémoire de nombreux Mauriciens, même si, comme le raconte un témoin, la raison de cette émeute est peu claire ${ }^{411}$. Jean Houbert explique que ces altercations restent certes floues ${ }^{412}$ et se seraient produites à cause de divergences politiques ${ }^{413}$ qui auraient causé des frictions entre créoles et musulmans et mené à une violence meurtrière ${ }^{414}$. Cet avis de Houbert semble être conforme au rapport de l'Electoral Institue for Sustainable Democracy in Africa qui confirme que ces conflits étaient en rapport aux communautés créoles et musulmanes ${ }^{415}$. Ces conflits violents entre créoles et musulmans ont pris la forme de «bagarres raciales » ${ }^{416}$; fait confirmé aussi dans le journal local Le Cernéen, qui avance que les conflits avaient éclaté «entre la communauté musulmane et la population générale ${ }^{417}$. Dans le roman, lorsque le docteur Bissam est arrêté par la police, ces derniers ont enfermé « les musulmans d'un côté, les créoles de l'autre » ${ }^{418}$ et

\footnotetext{
${ }^{410}$ Ananda Devi, Le Sari vert, Paris, Gallimard, 2009, p. 127.

411 « People believed at the time that this tense atmosphere had been instigated by some people, but the reason behind it is not very clear [...] To this day it has never been ascertained how it started-people would rather forget about it», Mauritiusmag, "An Eye Witness Account of the 1968 Riots », http://www.mauritiusmag.com/?p=598, consulté le 30 août 2013.

$\frac{112}{41 /}$ What was strange about the violence of 1968 was that it was between Creoles and Muslims, the two ethnic groups which had opposed independence, that it remained localised in a suburd of the capital, and that it occurred after the elections but before independence. Whatever the cause, one of the consequences was that the Muslims withdrew their support for a time for the P.M.S.D. », Jean Houbert, « Mauritius: Independence and Dependence », The Journal of Modern African Studies, 19, 1981, p. 87.

${ }^{413}$ Lorsque la question de l'acquisition de l'indépendance de l'île Maurice s'est posée, Le Parti mauricien social démocrate (P.M.S.D.), soutenu majoritairement par les Créoles mauriciens, était en faveur que l'île continue à être dirigée par l'Angleterre alors que le Mauritius Labour Party (M.L.P.) optait pour l'indépendance de l'île. C'est à ce moment que le P.M.S.D., dirigé par Gaëtan Duval, fit appel à tous les Mauriciens pour voter contre l'indépendance de l'île. Mais le régime britannique a décidé d'aller de l'avant en octroyant à l'île son indépendance. Cette décision sous-entendait aussi que la Couronne britannique devait tout faire pour que le M.L.P. reste au pouvoir à l'île Maurice. Ces conflits sociaux se sont produits en janvier 1968, juste avant que l'île obtienne son indépendance en mars 1968, Jean Houbert, « Mauritius: Independence and Dependence », The Journal of Modern African Studies, 19, 1981, p.75-105.

${ }^{414}$ « The coloured middle class sulked for a time, and a few even emigrated to Australia; the poor black Creoles and a number of Muslims vented their frustration in a short but murderous bout of communal violence in the capital, Port-Louis, just before independence », Ibid., p. 87.

415 «On 22 August 1966 the Legislative Assembly unanimously passed a motion requesting independence for Mauritius from United Kingdom, the Parti Mauricien walked out before the vote was taken [...] these were followed by ten days of murderous communal riots in January 1968 between Muslims and Creoles, a state of emergency was declared on 22 January and British troops shipped in from Singapore to suppress the violence [...] Muslims were alienated from the P.M.S.D. for several years thereafter », Electoral Institute for Sustainable Democracy in Africa, « Mauritius: the Road to Independence (1945- 1968)», http://www.content.eisa.org.za/old-page/mauritius-road-independence1945-1968, consulté le 1 juillet 2014.

${ }^{416}$ Ananda Devi, Le Sari vert, Paris, Gallimard, 2009, p. 128.

417 Anonyme, «Port-Louis. La situation s'aggrave », Le Cernéen, $\mathrm{n}^{\mathrm{o}}$ 5262, 22 janvier 1968, page inconnue.

${ }^{418}$ Ananda Devi, Le Sari vert, Paris, Gallimard, 2009, p. 130.
} 
le docteur avait été emprisonné avec « le groupe des musulmans » ${ }^{419}$ sans doute parce que son apparence physique était plus proche des musulmans que des créoles. Le Cernéen confirme aussi que ces conflits sociaux avaient majoritairement éclaté à PortLouis et «dans les localités de la Plaine Verte, de Roche Bois et de St Croix » ${ }^{420}$. George Thomson, le secrétaire du Commonwealth de l'époque, confirme aussi qu'il y a eu des séries d'altercations entre les groupes communaux dans l'est de Port-Louis ${ }^{421}$.

De surcroît, le médecin raconte que l'impact de ces émeutes sanglantes et meutrières était telles qu'il «arrivait sans cesse de nouveaux blessés qu'il fallait recoller d'urgence. Des gens à la tête fracassée, aux bras cassés, au nez éclaté ${ }^{422}$ au point que «les premiers morts étaient arrivés à la morgue [...] dans leurs vêtements baignés de sang ${ }^{423}$. Si le roman ne donne pas de précision sur le nombre de morts, Thomson indique qu'ils étaient dix-sept ${ }^{424}$ tandis que le rapport de l'Electoral Institute for Sustainable Democracy in Africa affirme que le nombre de morts était de vingtcinq $^{425}$, et Stanley Smith, pour sa part, affirme que le nombre en était vingt-sept ${ }^{426}$. Devi n'a, pour sa part, fourni aucune information détaillée sur ces conflits préférant décrire l'impact humain et social de ces conflits raciaux. D'après Vincent Jouve, cette technique d'omettre quelques informations plutôt que d'autres relève du fait que «l'énoncé doit contenir autant d'informations qu'il est requis mais pas plus qu'il est nécessaire ${ }^{427}$ et de ce fait, même si Devi ne précise pas et ne s'attarde pas sur le nombre de morts, le lecteur arrive quand même à cerner la gravité de la situation. Cette dernière se dégradait si rapidement qu'un état d'urgence avait été déclaré à Maurice,

\footnotetext{
419 Ibid.

420 Anonyme, «Port-Louis. La situation s’aggrave », Le Cernéen, nº 5262, 22 janvier 1968, page inconnue.

421 «There has been a series of brawls between communal gangs in Eastern Port-Louis », Commons and Lords Hansard, Commons Sitting of 23 January 1968, « Mauritius (State of Emergency) », http://hansard.millbanksystems.com/commons/1968/jan/23/mauritius-state-of-emergency, consulté le 30 août 2013.

${ }^{422}$ Ananda Devi, Le Sari vert, Paris, Gallimard, 2009, p. 127.

${ }^{423}$ Ibid., p. 128.

424 «Within the last half hour I have been in telephonic communication with the Governor. I regret to say that the death toll has now reached 17 », Commons and Lords Hansard, Commons Sitting of 23 January 1968, « Mauritius (State of Emergency)»,

http://hansard.millbanksystems.com/commons/1968/jan/23/mauritius-state-of-emergency, consulté le 30 août 2013.

425 « At least 25 people were killed, hundreds were wounded and thousands driven from their homes », Electoral Institute for Sustainable Democracy in Africa, « Mauritius: the Road to Independence (19451968) », http://www.content.eisa.org.za/old-page/mauritius-road-independence-1945-1968, consulté le 1 juillet 2014 .

426 «The rioting between Muslims and Creoles early 1968, resulting in twenty-seven deaths, was unprecedented », Stanley De Smith, « Mauritius: Constitutionalism in a Plural Society », The Modern Law Review, 31, 1968, p. 602.

${ }^{427}$ Vincent Jouve, L'Effet-Personnage dans le roman, Paris, Presses Universitaires de France, 1992, p. 100 .
} 
suivi de l'envoi de troupes britanniques pour rétablir l'ordre ${ }^{428}$. C'est lors d'une de ces soirées ensanglantées que le médecin, sortant de «l'hôpital civil » ${ }^{429}$ où il travaillait, allait être attaqué par les émeutiers mais il affirme avoir eu de la chance car «ce soir la Special Mobile Force est arrivée avant qu'ils réussissent à s'entre-tuer. Les militaires les ont arrêtés ${ }^{430}$; la Special Mobile Force, l'armée mauricienne, créée en $1960^{431}$ avait pour but d'assurer la sécurité du pays ${ }^{432}$.

Bien que le médecin ne soit qu'une victime innocente, les policiers et la Special Mobile Force l'ont emmené avec les émeutiers «aux casernes » ${ }^{433}$ de la police mauricienne situées à Port-Louis. En effet, lors des conflits, la SMF et la police mauricienne faisaient leur mieux pour calmer la situation et «de fortes patrouilles mobiles [avaient] opéré dans les régions affectées durant toute la nuit du 22 janvier. Quinze personnes [avaient] été arrêtées et détenues par la police » ${ }^{434}$. La SMF avait été récemment mise en place, mais n'avait pas les moyens et le grand nombre de soldats nécessaires pour riposter ${ }^{435}$. C'est exactement la situation qu'a vécue le docteur Bissam qui note que : «les slogans haineux retentissaient et les mots se chargeaient de plus en plus d'un goût acide ${ }^{436}$ et que la police et les soldats mauriciens « sillonnaient le pays sans parvenir à masquer leur impuissance ${ }^{437}$. D'après le journal Week-end, ce sont les hélicoptères de la marine britannique qui ont aidé la police mauricienne dans une grande mesure à localiser les rassemblements et à empêcher d'autres actes de violence $^{438}$.

\footnotetext{
428 «Mr. Braine (by Private Notice) asked the Commonwealth Secretary whether he will make a statement about the declaration of a state of emergency in Mauritius and the sending of British troops from Singapore to restore order », Commons and Lords Hansard, Commons Sitting of 23 January 1968, « Mauritius (State of Emergency)», http://hansard.millbanksystems.com/commons/1968/jan/23/mauritius-state-of-emergency, consulté le 30 août 2013.

${ }^{429}$ Ananda Devi, Le Sari vert, Paris, Gallimard, 2009, p. 130.

${ }^{430}$ Ibid.

${ }^{431}$ Special Mobile Force, «Presentation of Regimental Colour on the occasion of the $50^{\text {th }}$ Anniversary of the SMF », SMF Gymkhana Grounds, Vacoas.

432 «On the departure of the British Garrison, the Mauritius Special Mobile Force was formed. It was evident that the task of the newly created SMF was to ensure the internal security of the country », Special Mobile Force, «Presentation of Regimental Colour on the occasion of the $50^{\text {th }}$ Anniversary of the SMF ", SMF Gymkhana Grounds, Vacoas.

${ }_{433}$ Ananda Devi, Le Sari vert, Paris, Gallimard, 2009, p. 130.

${ }^{434}$ Anonyme, «Port-Louis. La situation s'aggrave», Le Cernéen, nº 5262, 22 janvier 1968, page inconnue.

${ }^{435}$ C'est pour cela que le régiment britannique, connu aussi comme le King's Shropshire Light Infantry, était mandé sur l'île sous la commande du Major Brian Lowe, Mauritiusmag, « An Eye Witness Account of the 1968 Riots », http://www.mauritiusmag.com/?p=598, consulté le 30 août 2013.

${ }_{436}^{436}$ Ananda Devi, Le Sari vert, Paris, Gallimard, 2009, p. 128.

${ }^{437}$ Ibid.

${ }^{438}$ Anonyme, « 20 morts, 212 blessés. Le bilan d'une semaine de violence », Week-end, nº 70, 28 janvier 1968 , page inconnue.
} 


\section{Anachronismes du présent diégétique et faits historiques à la fois locaux et universels dans Le Sari vert}

En somme, dans Le Sari vert, les références historiques à la période de l'esclavage, à l'épisode où de nombreux esclaves affranchis se sont jetés de la falaise du Morne afin d'échapper à la maltraitance et de retrouver la liberté, aux grèves de 1937 des petits planteurs de cannes à sucre, et aux émeutes qui ont éclaté dans l'île en janvier 1968, deux mois avant l'indépendance, entre les communautés créole et musulmane de l'île Maurice rendent l'histoire du médecin plus réelle, vivante et crédible. En même temps, ces faits sont fictionnalisés et interprétés pour mettre l'accent sur leur signification sociale. Par ailleurs, il a été possible de situer le présent du récit dans la deuxième moitié des années 1980. Nous avons également détecté un anachronisme important concernant la femme du docteur. Cet anachronisme, qui constitue un amalgame des émeutes en 1968 et des grèves des petits planteurs de 1937, a pour effet d'accentuer l'importance qu'ont eue les idées d'émancipation dans l'histoire de la société mauricienne.

\section{Cadre réel local et problèmes universels dans les trois romans}

L'étude détaillée des références temporelles et des événements historiques ont mis en avant des périodes différentes de l'histoire mauricienne. Dans Rue la Poudrière, l'histoire de Paule se situe entre 1959 à 1979, et il est fait mention de la grève des dockers qui s'est produite à l'île Maurice en 1979 et de la démolition des bâtiments insalubres de la Butte qui a commencé dans les années 1970. Dans Le Voile de Draupadi, la mort du fils d'Anjali se situe en 1986, et il est question du passé lointain du grand-père d'Anjali arrivé comme travailleur engagé entre 1920 et 1924. Dans Le Sari vert, l'agonie du docteur Bissam se situe dans la première moitié des années 1980 et il est fait mention de deux incidents historiques majeurs qui ont secoué la population mauricienne : la grève des coupeurs de canne de l'hiver 1937 et le conflit racial de janvier 1968 entre musulmans et créoles.

Dans les trois romans, les événements historiques sont si bien représentés et détaillés, qu'ils contribuent à l'effet de réel ainsi qu'à mettre en avant leur signification sociale. De fait, les descriptions réalistes de ces événements historiques ancrent l'histoire des trois romans dans un contexte historique résolument mauricien. 
Si la grève de 1979, dans Rue la Poudrière, reflète une réalité historique mauricienne, ce n'est pas pour autant le seul pays qui a connu des grèves de débardeurs ou de coupeurs de canne qui cherchaient à améliorer leur situation de travail et leur salaire. Un des exemples serait la grève des débardeurs à Londres en 1889, dont a discuté Henry Champion ${ }^{439}$, et un autre serait celle qui s'est produite aux Etats Unis en 1913, dont a parlé Dan Radnika ${ }^{440}$. Aussi, les conditions des dockers et des coupeurs de canne sont universelles tout comme leurs revendications.

De la même façon, si Le Voile de Draupadi décrit la traversée entre l'Inde et Maurice, cette dernière n'est certainement pas la seule île à avoir accueilli des travailleurs engagés indiens. En effet, sous la colonisation britannique qui avait débuté tôt dans les années 1800, les travailleurs engagés indiens, étaient expédiés dans d'autres colonies telles l'île de la Réunion, les îles Fidji, le Surinam, la Guyane, Trinidad, les Seychelles, la Jamaïque, et l'Afrique du $\mathrm{Sud}^{441}$. Les conditions rudes des travailleurs engagés de l'Inde à destination des colonies mentionnées ont été évoquées dans plusieurs documents historiques comme le rappelle «The Passage » du texte A New System of Slavery de Hugh Tinker ${ }^{442}$, David Northrup ${ }^{443}$ ou Gaiutra Bahadur ${ }^{444}$. Tous démontrent des conditions de voyage similaires à celles décrites dans le roman ${ }^{445}$. De plus, l'historien David Northrup compare la traversée des coolies aux voyages des Chinois qui n'hésitaient pas non plus à mettre fin à leur vie ${ }^{446}$, ou au long voyage des migrants irlandais qui partaient vers l'Amérique du $\operatorname{Nord}^{447}$. Ceci démontre à quel point ce genre de déplacement n'est pas confiné aux frontières de l'île Maurice. Ce thème du voyage et de l'exil des travailleurs évoqué par l'entremise du grand-père d'Anjali

\footnotetext{
${ }^{439}$ Henry Champion. The Great Dock Strike: in London, August, 1889, London, Swan Sonnenschein \& Co, 1890 .

${ }^{440}$ Dan Radnika, « 100 Years Ago: the Philadelphia dockers strike and Local 8 of the IWW », http://libcom.org/library/100-years-ago-philadelphia-dockers-strike-local-8-iww-mouvementcommuniste-kolektivn\%C4\%9B-pr, consulté le 12 novembre 2013.

${ }^{441}$ Girmitunited.org, « Colonial British Indian Indentured Labour Transportation by Country », http://girmitunited.org/girmit/?page_id=816, consulté le 13 novembre 2013.

${ }^{442}$ Hugh Tinker, A New System of Slavery, London, The Camelot Press Ltd, 1974.

${ }^{443}$ David Northrup, Indentured Labour in the Age of Imperialism, 1834-1922, Cambridge, Cambridge University Press, 1995.

${ }^{444}$ Gaiutra Bahadur, Coolie Woman. The Odyssey of Indenture, Chicago, The University of Chicago Press, 2014.

${ }^{445}$ Pour de plus amples détails sur les conditions de la traversé des travailleurs engagés, voir la section « Ouvrages sur l'histoire de l'île Maurice consultés » de la bibliographie.

446 «Under these appalling circumstances some Chinese migrants sought to escape through suicide », David Northrup, Indentured Labour in the Age of Imperialism, 1834-1922, Cambridge, Cambridge University Press, 1995, p. 86.

${ }_{447}$ «Conditions of Irish migrants en route to North America in 1881 could as well apply to most indentured laborers $[\ldots]$ most would have been uncertain and confused about what lay before them ", Ibid., p. 88.
} 
remonte en effet à la traite des esclaves. Si les travailleurs engagés indiens étaient dupés et bernés pour qu'ils entreprennent la traversée, les esclaves africains et malgaches étaient, eux, enlevés de force de leur pays natal pour aller à l'île Maurice, comme l'expliquent Satyendra Peerthum et Richard Allen ${ }^{448}$. L'expérience du grandpère d'Anjali est représentative de celle de nombreux travailleurs exilés dans le monde entier.

Pareillement, dans Le Sari vert, si les émeutes des coupeurs de cannes à sucre de 1937 et le conflit racial de janvier 1968 ont frappé l'île Maurice, les mêmes atrocités existent encore de nos jours. Plusieurs endroits dans le monde sont les plateformes de ces conflits dont la population souffre ${ }^{449}$. De ce fait, ce genre de conflits ne se confinent pas à l'île Maurice mais sont universels.

Finalement, les trois romans à l'étude se situent dans un cadre historique mauricien très réaliste basé sur le passé réel et douloureux de l'île Maurice. Toutefois, la façon dont sont décrits ces événements, que ce soit la grève des travailleurs, les conflits raciaux, la traversée des coolies et leurs conditions de vie, conduit à une lecture beaucoup plus universelle. Devi s'est servie de l'histoire mauricienne pour représenter des faits universels. De ce fait, l'auteure prouve de nouveau que ses romans peuvent très bien être casés dans un cadre local mais que les thèmes abordés dépassent sans aucun doute les frontières de son île natale.

\footnotetext{
${ }^{448}$ Satyendra Peerthum, « The Horror of Slave Voyage to Mauritius Remembered », http://www.lexpress.mu/article/horror-slave-voyage-mauritius-remembered, consulté le 24 septembre 2013 ; Richard Allen, «Licentious and Unbridled Proceedings: the illegal Slave Trade to Mauritius and the Seychelles during the Early Nineteenth Century », The Journal of African History, 42, 2001, p. 91116.

${ }^{449}$ Pour de plus amples détails sur la discrimination et les conflits raciaux, voir : Robert Sellers, Cleopatra Caldwell, Karen Schmeelk-Cone, Marc Zimmerman, « Racial Identity, Racial Discrimination, Perceived Stress, and Psychological Distress among African American Young Adults », Journal of Health and Social Behavior, 44, 2003, p. 302-317 ; Tony Brown, David Williams, James Jackson, Harold Neighbors, Myriam Torres, Sherrill Sellers, Kendrick Brown, « Being Black and Feeling Blue: the Mental Health Consequences of Racial Discrimination », Race \& Society, 2, 2000, p. 117-131 ; Saffron Karlsen, James Nazroo, «Relation Between Racial Discrimination, Social Class, and Health Among Ethnic Minority Groups », American Journal of Public Health, 92, 2002, p. 624-631.
} 


\section{Partie IV}

\section{Dimension sociale}




\section{Introduction}

Dans ce chapitre, nous proposons d'examiner, en nous appuyant principalement sur les travaux des théoriciens tels Emile Durkheim et Robert Merton, et de sociologues et statisticiens, surtout mauriciens, la situation sociale des personnages et les conditions de vie dépeintes dans les trois romans pour d'abord confronter leur situation à la réalité mauricienne et déterminer à quel point cette société est perçue avec réalisme. Par ailleurs, puisque deux des trois romans sont centrés sur la communauté hindoue et la disparité qui existe entre les communautés hindoue et créole, l'accent sera également mis sur la notion de coolitude de Khal Torabully.Ensuite, nous évaluerons dans quelle mesure cette réalité est spécifiquement mauricienne, ou au contraire universelle.

La société de l'île Maurice est complexe et englobe différentes communautés qui essayent de cohabiter tant bien que mal : les Créoles, les Hindous, les Musulmans, les Sino-Mauriciens et les Européens sont les cinq groupes principaux qui la composent. Si Rue la Poudrière est axé sur la communauté créole, et en particulier sur la classe ouvrière en dépeignant la relation chaotique d'Edouard et Marie, Le Voile de Draupadi dépeint, par l'intermédiaire du couple Dev et Anjali, les rites et croyances de la communauté hindoue mais aussi la disparité qui existe entre cette dernière et la communauté créole. Le Sari vert, tout comme Le Voile de Draupadi, dépeint la classe moyenne hindoue et la disparité entre les communautés hindoue et créole par le biais du docteur Bissam et de sa femme.

À part étudier l'appartenance ethnique des personnages et les disparités sociales, entendues dans un sens très large, entre ces groupes sociaux, ce travail se tournera vers les rapports de couple entre les personnages masculins et féminins, car ceux-ci dévoilent souvent les attitudes typiques de l'homme et de la femme à l'intérieur d'un groupe social donné. Dans Rue la Poudrière, nous examinerons les couples Marie et Edouard, Paule et Mallacre, Paule et Tapsy, alors que dans Le Voile de Draupadi, l'accent sera mis sur les parents d'Anjali, sur Anjali et Dev, et sur Margaret et Shyam. Finalement, dans Le Sari vert, ce sont les couples du docteur et de sa femme, de Kitty et de son mari, et de Malika et de Marie-Rose qui seront examinés. 


\section{Rue la Poudrière}

Avant de mener à bien l'étude proprement dite des communautés dépeintes et du rapport dans les couples décrits dans Rue la Poudrière, il importe de faire le survol des recherches faites sur ce livre.

Vicram Ramharai, qui étudie dans Rue la Poudrière la question de l'identité de la femme mauricienne ${ }^{450}$, met en avant la souffrance de la femme, dont l'homme, même s'il souffre, est responsable. Selon lui, la libération de la femme ne peut se faire que par «une éducation de l'homme mauricien ${ }^{451}$. De plus, il remarque que cette situation difficile de la femme «n'est certes pas exclusive à l'île Maurice mais sa particularité d'être un pays multiracial fait que la situation de la femme y est plus complexe qu'ailleurs ${ }^{452}$. Il ajoute que :

Les époux et les pères, peu présents, sont, peut-être, les plus touchés par ces drames. En tournant le dos à leur fille ou à leur épouse, ils sont largement responsables de ce qui leur arrive. Le poids de la responsabilité envers la fille indésirée ou l'épouse que l'on ne peut pas ou ne plus aimer, constitue une catastrophe pour ces hommes ${ }^{453}$.

Dans une étude consacrée partiellement à Rue la Poudrière, Bannerjee ${ }^{454}$ se concentre surtout sur le rôle de la femme créole en contraste avec celui de l'IndoMauricienne en mettant en avant la vie turbulente et nomadique des prostituées, pour la plupart créoles. Elle ajoute qu'en jouissant d'une vie sexuelle épanouie, Paule fait exactement ce que la femme hindoue ne doit pas faire ${ }^{455}$. Paule représente donc celle qui refuse de se soumettre aux règles patriarcales et symbolise le courage et

\footnotetext{
450 «L'œuvre d'Ananda Devi se caractérise par son importante contribution à la construction d'une identité de la femme mauricienne », Vicram Ramharai, « Ananda Devi : repenser l'identité de la femme mauricienne », Notre librairie, 146, 2001, p. 110.

${ }^{451}$ Ibid., p. 106.

452 Ibid., p. 107.

${ }^{453}$ Ibid., p. 105.

${ }^{454}$ Rohini Bannerjee, «Daughter Forsaken: la résistance of the Indo-Mauritian Girl Child in Ananda Devi's Novel » dans India in the World, Cristina M. Gámez-Fernández, Antonia Navarro-Tejero, Newcastle, Cambridge Scholars Press, 2011, p. 123-133.

455 « Typical of Devi's female protagonists, Paule does not succumb to the idea of the girl child prostitute as further victimization, despite the violence experienced both in the home and on the streets of PortLouis. Instead, she considers herself not only as a form of relief and psychological catharsis from the burdens of domestic poverty for her clients but also for girl child status; becoming a prostitute actually helps Paule escape the exigencies of playing the ideal Hindu daughter », Ibid., 2011, p. 126.
} 
l'émancipation ${ }^{456}$. Eileen Lohka, elle aussi est d'avis que Paule, « qui semblent naître directement du terreau de son île ${ }^{457}$, se trouve dans la catégorie des nomades « dont la vie ne laisse que peu de traces ${ }^{458}$, et que Mallacre représente celui qui contrôle ces nomades. Elle conclut que malgré leur noirceur, les romans de Devi, ancrés « dans la mémoire et dans le temps ${ }^{459}$, garantissent l'évasion. De son côté, Ashwiny Kistnareddy ${ }^{460}$ constate que les Créoles souffrent d'un désintérêt pour leur passé ${ }^{461}$, et selon elle, Marie représente à la fois la femme destructrice qui pratique la magie noire et la femme protectrice, la Vierge Marie ${ }^{462}$. Kistnareddy conclut que, dans la société mauricienne que dépeint Devi, il y a deux modèles de créolisation : celui régi par l'histoire et ses sources, et celui régi par la perte d'identité ${ }^{463}$. Pour sa part, Njeri Githire $^{464}$, se donnant d'étudier la nutrition dans Rue la Poudrière, avance que la malnutrition agit comme des barrières qui créent des distinctions sociales ${ }^{465}$.

Ritu Tyagi, pense que les personnages femmes de Rue la Poudrière vivent dans un monde de désespoir, destitution, folie, putréfaction et destruction, un monde noir et statique $^{466}$ où les femmes ne seraient pas considérées comme humaines, mais plutôt comme des ombres ${ }^{467}$.

\footnotetext{
456 « Devi's girl child protagonists shun a simplified representation of themselves and refuse to submit to the conditions of patriarchal control, but instead resist in the given space allowed to them, be it the streets of Port-Louis or the behind the living-room hearth. Under Devi's pen, Paule and Mouna become IndoMauritian women and symbols of courage and emancipation », Ibid., p. 131.

${ }^{457}$ Eileen Lohka, « De la terre à la terre, du berceau à la tombe, lîle d'Ananda Devi », Nouvelles études francophones, 23, 1, 2008, p. 157.

${ }^{458}$ Ibid., p. 156.

${ }^{459}$ Ibid., p. 162.

${ }^{460}$ Ashwiny Kistnareddy, «Interrogating Identity: Psychological Dislocations in Ananda Devi's Novels », Dalhousie French Studies, 94, Spring 2011, p. 27-38.

${ }^{461}$ «The Indo-mauritian community and the Creole community are marked by over-attachment to the past and loss of past, respectively », Ibid., p. 27.

462 «Images of splits and doubles recur in this narrative where the mother herself is double: Marie is the longaniste and the baby killer and Marie the Virgin who protects her son. In the name of Marie, there is thus the double signifier », Ibid., p. 34.

${ }^{463}$ «In Mauritian society as depicted by Devi, there are two different models that impede the notion of creolisation and the richness it entails: one focusing on history and roots and the other having lost its memory. If the question of identity brings about such suffering as has been analysed in this article, then Devi suggests, we should not persist in our quest but should simply be », Ibid., p. 36-37.

${ }^{464}$ Njeri Githire, «The Semiotics of (Not) Eating: Fasting, Anorexia, and Hunger Strike in Ananda Devi's Le Voile de Draupadi », Nottingham French Studies, 48, 1, 2009, p. 82-93.

465 «In this novel [Rue la Poudrière], food is presented as a marker of social distinctions and boundaries, a marker which Paule attempts to erase as she seeks to escape from a strongly rooted identity », Ibid., p. 85 .

${ }^{466}$ «Pagli, La Vie de Joséphin le fou, Eve de ses décombres, L'Arbre fouet, and Rue la Poudrière introduce the reader to a mysterious world of despair, destitution, madness, putrefaction and destitution », Ritu Tyagi, «"Feminine" Desire in Ananda Devi's Narratives », Dalhousie French Studies, 94, 2011, p. 65.

${ }^{467}$ «In fact, society's rejection of these protagonists is so extreme that they are no longer perceived as human beings, but rather as shadows. Their worlds are somber and dark; their tales, slow and static », Ibid.
} 
Rohini Bannerjee, se concentrant cette fois-ci sur la question de la langue, s'interroge sur l'utilisation du créole dans les romans de Devi, et surtout dans Rue la Poudrière. Elle est d'avis que le créole permet de préserver « une sorte de nostalgie de la vie mauricienne de ces personnages ${ }^{468}$. Si Devi réécrit en français les phrases en créole, c'est pour «clarifier le sens du créole » ${ }^{469}$ et aussi pour «préserver l'environnement socio-culturel dans lequel elle écrit ${ }^{470}$. Devi contribue ainsi à une «francophonie universelle » ${ }^{471}$ et Soupirs et Rue la Poudrière sont «deux textes [...] qui traduisent une libération littéraire où la plume dévidienne imprime le créole dans le langage du quotidien, derrière chaque virgule du français standard ${ }^{472}$.

Nous constatons que beaucoup de ces analystes se sont intéressés à la condition misérable de la femme dans une société mauricienne hautement patriarcale et inégale sans traiter des relations entre homme et femme au sein du couple. En outre, s'ils s'intéressent à l'origine sociale des personnages, ils n'étudient pas la disparité qui existe pour la communauté créole. Dans cette partie, nous visons à étudier les différentes facettes de la société dépeinte dans Rue la Poudrière, et plus précisément l'origine ethnique et la classe sociale des personnages, les langues utilisées, et les relations au sein des couples Marie et Edouard, Paule et Mallacre, et Paule et Tapsy, pour déterminer si le roman reflète bien la réalité mauricienne et pour voir si la situation de ces personnages est unique à l'île Maurice.

\section{La communauté créole dans Rue la Poudrière}

Rue la Poudrière dépeint la situation d'une famille créole qui évolue dans un cercle également créole. Ainsi, les personnages portent des noms typiques de cette classe : Paule, Marie, Edouard, Mireille, Aline. De plus, vu que Paule, âgée de quatre ans, accompagne sa mère pour se rendre à la chapelle de la rue la Paix à Port-Louis tous les dimanches ${ }^{473}$, la famille est catholique, à l'instar des Créoles. La situation précaire de ces personnages créoles est frappante ; pauvreté, insalubrité, manque d'éducation et statut social défavorisé sont des traits attachés à la famille de Paule. Par ailleurs, tous ces personnages créoles sont dépeints comme répugnants. Ainsi, Marie est décrite

\footnotetext{
${ }^{468}$ Rohini Bannerjee, «L'Espace littéraire mauricien : l'hétérolinguisme dans l'œuvre francophone d'Ananda Devi », Contemporary French and Francophone Studies, 15, 2011, p. 507.

${ }^{469}$ Ibid., p. 508.

${ }^{470}$ Ibid., p. 508.

${ }^{471}$ Ibid., p. 506.

${ }^{472}$ Ibid.

473 « Je me revoyais, à quatre ans, heureuse de marcher le long de la rue la Paix avec ma mère jusqu'à la chapelle, les dimanches », Ananda Devi, Rue la Poudrière, Abidjan, Nouvelles Editions Africaines, 1988 , p. 8.
} 
comme « noire ${ }^{474}$, « vaste et écrasante ${ }^{475}$, ayant « une odeur de soufre comme si des plaques de son corps avait été brûlées au soleil » ${ }^{476}$. C'est une «masse de chair vibrante ${ }^{477}$ avec de $«$ grosses lèvres et (un) nez caverneux ${ }^{478}$. Quant à Edouard, il a une «face épaisse et osseuse ${ }^{479}$ et un « laid visage [...] [avec des] dents jaunes ${ }^{480}$. Mallacre a « un œil mince et jaune ${ }^{481}$, des «dents irrégulières ${ }^{482}$ et le père de Mireille, amie de Paule, a « une tête étroite et monstrueuse [...] une figure d'un noir opaque, des lèvres pourpres $»^{483}$.

Cette représentation négative des personnages créoles reflète-t-elle un stéréotype au sein de la société mauricienne ? D’après Lionnet, Devi «creuse inlassablement depuis plus de trente ans, [le thème] de l'exclusion et de ses contrecoups ${ }^{484}$ parce que $«$ le passé colonial n'est pas mort ${ }^{485}$. Aussi, Devi par l'entremise de ses personnages, dénonce les «inégalités qui perdurent en dépit de l'indépendance, et qui vont en s'accroissant aujourd'hui à cause de la mondialisation ${ }^{486}$, ainsi que les préjugés qui maintiennent la communauté créole en bas de l'échelle sociale. A en croire Sandra Carmignani, le terme «créole », dans le contexte mauricien renvoie à l'esclavage : «renvoyé[s] à la violence de l'esclavage et au déni de la créolisation, leurs origines sont perçues comme refoulées, oubliées, incertaines ${ }^{487}$ et la communauté est dénigrée par les autres à cause de ses origines. Pour sa part, Danielle Tranquille pense que les Créoles mauriciens incluent aussi ceux qui ont des origines et traditions mixtes africaines, asiatiques ou françaises, et ceux qui sont les descendants d'Indiens qui se sont convertis au christianisme ${ }^{488}$. Thomas Eriksen estime que les Créoles mauriciens sont les descendants africains ou

\footnotetext{
474 Ibid.

${ }^{475}$ Ibid., p. 11.

476 Ibid.

${ }^{477}$ Ibid., p. 12.

${ }^{478}$ Ibid., p. 55

${ }^{479}$ Ibid., p. 16

${ }^{480}$ Ibid., p. 94.

${ }^{481}$ Ibid., p. 88.

482 Ibid., p. 104.

${ }^{483}$ Ibid., p. 20.

${ }^{484}$ Françoise Lionnet, « Matière à photographie : Cosmopolitique et modernité créoles à l'île Maurice », French Forum, 34, 2009, p. 87.

${ }^{485}$ Ibid., p. 93.

${ }^{486}$ Ibid., p. $91-92$.

${ }^{487}$ Sandra Carmignani, «Figures identitaires créoles et patrimoine à l'île Maurice : une montagne en jeu », Journal des anthropologues, 2006, p. 268.

488 «Who are the créoles? Are they solely of African descent? What about those who are of mixed backgrounds: African, Asian and/or French descent? Or those others still, who are of Indian ancestry and have accepted Christian faith?», Danielle Tranquille, "Inscriptions of dev/fiancé: métissage in Mauritian literature », International Journal of Francophone Studies, 8, 2005, p. 203.
} 
malgaches ${ }^{489}$ dont le déracinement et la connexion avec leur lieu d'origine, les placent dans la même situation que les Hindous ${ }^{490}$.

A cause du manque de recherches sociologiques sur les années 1959-1979, nous proposons de nous baser sur les chiffres récents pour analyser la situation des personnages créoles du roman ${ }^{491}$. Ces chiffres, bien que trop récents, permettent d'établir des tendances qui sous-tendent la société mauricienne depuis l'indépendance. Pour les psycho-anthropologues et les historiens de la commission Truth and Justice publié en 2011, la situation actuelle des Créoles mauriciens et le traitement réservé aux descendants d'esclaves par les Mauriciens, est le résultat d'une déculturation profonde et inconsciente qui a fini par créer des discordes et des conflits ${ }^{492}$. D'après François Paul, « la situation de la communauté créole semble être confuse, et la voie de la promotion sociale encore plus complexe ${ }^{493}$. Il estime aussi que ce sont l'Eglise catholique et les partis politiques qui sont coupables de ne pas aider les Créoles à évoluer :

L'autorité de l'Eglise catholique a pour ainsi dire laissé pourrir la situation de la communauté créole, et toujours pratiqué l'égocentrisme qui lui est propre [...]. D'autre part, les partis politiques à vocation créole ont toujours cherché à assouvir leur soif de pouvoir, comme des sanguinaires en puisant leur force dans l'électorat créole $^{494}$.

\footnotetext{
489 «It is traditionally the Mauritians of African and/or Malagasy descent who are classified locally as Creoles », Eriksen Hylland, «Tu Dimunn Pu Vinn Kreol: the Mauritian Creole and the Concept of "Creolization", 1999, http://folk.uio.no/geirthe/Creoles.html, consulté le 13 avril 2012.

${ }^{490}$ «Like other groups known as Creoles, Mauritian Creoles have a history of uprootedness and the connection with their places of origin was severed almost immediately upon arrival in the colony », Ibid.

${ }^{491}$ Ce manque peut s'expliquer par le fait que ces sujets étaient, à l'époque, tabous ou bien considérés comme normaux.

${ }^{492}$ « There have been several interpretations of this state of affairs for this ethnic group, but many psycho-anthropologists and historians trace it back to slavery and its aftermath. The treatment which they were subjected to and their deculturalization appear to be deeply ingrained in their sub-consciousness [...] Such non-conscious phenomena affect individual functioning and become a source of strain, discord and strife », Truth \& Justice Commission, « Report of the Truth and Justice Commission. Volume 4. History, Economy, Society and Memory. Research Reports, Technical Studies and Surveys », http://pmo.gov.mu/English/Documents/TJC Vol4modified.pdf, consulté le 30 mars 2014, p. 514.

${ }^{493}$ Paul François, «La Communauté créole de l'île Maurice », http://csmsmagazine.org/?p=1667, consulté le 29 octobre 2013.

${ }^{494}$ Ibid.
} 
La description des personnages créoles de Rue la Poudrière et leur situation subalterne reproduisent bien les préjugés de la société mauricienne vis-à-vis des Créoles. Devi expose un état de fait réel mais n'offre pas d'explications quant à la situation inférieure du Créole mauricien.

\section{La classe inférieure dans Rue la Poudrière}

La famille de Paule fait partie de la classe inférieure où, traditionnellement, le mari travaille pour nourrir les siens, et où il est le chef de la famille qui prend les décisions majeures. Mais dans ce roman, c'est Marie qui se montre la plus forte et elle n'hésite pas à violenter son mari. De plus, c'est elle qui pourvoit aux besoins et devient, chef de famille à part entière, après l'accident de travail de son mari qui mène au chômage.

Lorsqu'il travaillait comme docker, Edouard dépensait tout son salaire dans des beuveries avec ses amis. Ceci est typique, d'après Stephen Sanderson, de la classe des travailleurs où l'homme préfère généralement être en compagnie d'autres hommes et se retrouver dans les bars ${ }^{495}$. Mais si Edouard est alcoolique, ce n'est pas sans raison. Son travail rude et son statut social inférieur qui ne lui permettent aucune échappatoire le poussent à se soûler. Un journal quotidien mauricien, faisant référence aux dockers, écrit que «leurs métiers, leurs conditions de vie et l'environnement ne leur offraient aucune meilleure perspective possible ${ }^{496}$. Margaret, une ancienne résidente des Dockers' Flats de Roche-Bois, témoigne de la déchéance de ces travailleurs : « je pense aussi que la vie des dockers était tellement dure que certains d'entre eux avaient sombré dans l'alcoolisme. Puis, leurs enfants ont manqué d'encadrement, à tous les niveaux, pour pouvoir éviter la déchéance ${ }^{497}$. Typiquement, Edouard, d'abord docker puis chômeur, répétait, au travail, les mêmes mouvements avec une régularité monotone et mécanique ${ }^{498}$, ce qui le pousse ensuite à l'alcoolisme puis à une absence de moralité, phénomène que Durkheim nomme anomie. Edouard est alcoolique parce qu'il est pauvre et désenchanté. Comme l'explique Merton, la personne frustrée et

\footnotetext{
${ }^{495}$ Stephen Sanderson, Macrosociology. An Introduction to Human Societies, New York, Harper \& Row Publishers, 1988, p. 349.

${ }^{496}$ Pradeep Daby, «Des Dockers Flats aux nouvelles résidences. Ce qui a changé pour des familles entières »,

http://www.defimedia.info/defi-plus/dp-societe/item/55709-des-dockers-flats-aux-nouvelles-residencesce-qui-a-change-pour-des-familles-entieres.html, consulté le 8 juin 2014.

${ }^{497}$ Ibid.

498 «Every day he repeats the same movements with monotonous regularity, but without having any interest or understanding of them », Emile Durkheim, The Division of Labour in Society, London, Macmillan Publishers, 1984, p. 306.
} 
inapte qui n'arrive pas à s'adapter abandonne et renonce à ses responsabilités ${ }^{499}$. Edouard, qui se réfugie dans l'alcool après avoir perdu son emploi en est l'exemple parfait.

D'autre part, les débardeurs, insatisfaits de leurs conditions de travail et n'ayant pas obtenu l'augmentation salariale réclamée, décident de faire grève. Exactement comme l'explique Durkheim; le simple mécontentement suffit pour qu'il y ait boycottage $^{500}$ et c'est exactement ce que Devi suggère dans son roman par le biais de ses personnages tels que Marie, Edouard et Mallacre. En somme, la famille de Paule est à l'image de la classe créole pauvre qui, d'après Joel Charon, est impuissante à contrôler sa situation économique, dépendante des autres pour sa survie et n'a presque pas d'impact sur l'ordre économique ou politique ${ }^{501}$.

Cette précarité ne permet pas à Edouard de réaliser son rêve mauricien. Si certains, comme Tapsy ou Edouard, n'aspirent qu'à travailler pour survivre, d'autres, tels que Mallacre ou Marie parviennent à avoir plus d'argent au prix de certains compromis. Marie n'hésite pas à s'adonner à la magie noire ${ }^{502}$ ou même à voler ${ }^{503}$ et Mallacre est proxénète. Le milieu où évolue Paule représente donc le microcosme d'un groupe social dysfonctionnel et mal considéré, celui des Créoles qui sont en bas de l'échelle sociale mauricienne.

\section{Utilisation des langues dans Rue la Poudrière}

Dans Rue la Poudrière, l'histoire est narrée en français standard et le personnage principal n'utilise que le français. Le hindi est utilisé une seule fois par Ganeshi, une femme folle de la communauté hindoue que Mallacre a aidée. Celle-ci chante une berceuse à un enfant imaginaire, en répétant les mêmes mots : «Soja baba, soja baba, soja... » ${ }^{504}$. D’autres personnages, tels que Marie, Edouard, Mallacre ou les

\footnotetext{
499 « The competitive order is maintained but the frustrated and handicapped individual who cannot cope with this order drops out », Robert Merton, Social Theory \& Social Structure, New York, The Free Press, 1968, p. 207.

500 " The slightest cause of discontent was enough to cause a firm to be boycotted », Emile Durkheim, The Division of Labour in Society, London, Macmillan Publishers, 1984, p. 293.

501 «One thing we know about the poor in all societies is that they are powerless to control their economic destiny, they are dependent on others for survival and they have almost no impact on the direction of the economic and political order », Joel Charon, The Meaning of Sociology, New Jersey, Prentice-Hall, 1987, p. 108.

502 « rentrant le soir, le visage creux, des billets et des pièces de monnaie boursouflant son cabas », Ananda Devi, Rue la Poudrière, Abidjan, Nouvelles Editions Africaines, 1988, p. 39.

503 « une chaîne en or, munie d'une croix, à son cou », Ibid., p. 72.

504 «Dors, bébé, dors... », Ibid., p. 174.
} 
prostituées ont de temps en temps recours à un langage issu de la classe inférieure peu éduquée qui constitue, à Maurice, la langue parlée courante commune, un «parler créole, lingua franca du pays [qui] évolue avec la scolarisation et se francise ${ }^{505}$. La mère de Paule utilise notamment le créole pour pratiquer la magie noire et évoquer le mal : «to pou touille li ? li pou fini mort, pauv'boug ! ${ }^{506}$. Elle l'utilise assez souvent lorsqu'elle s'adresse aux amis ivrognes d'Edouard : «Allez, sorti là allé ! Pas vinne faire désordre dans mo lakaze, mauvais bâtards ! ${ }^{507}$. De plus, une des prostituées de la maison close exprime ses sentiments envers Mallacre en créole : «Mallacre, ça ène boug ça! ène vrai boug! ${ }^{508}$. Une des lavandières s'exclame en créole «Ayo bon Dié ! qui été ça ? [...] li finne fini mort » ${ }^{509}$ lorsqu'elle découvre le corps inerte d'Aline. En fait, le langage des femmes issues de la classe des travailleurs est, d'après Susan Hughes, une langue vernaculaire qui est souvent associée à la masculinité et à la résistance $^{510}$, à l'image des personnages femmes qui utilisent le créole dans le roman. L'utilisation de ce type de langue au vocabulaire appauvri montre un manque d'éducation. A l'île Maurice, d'après la sociologue Sheila Bunwaree, si le gouvernement mauricien a aboli les frais d'examen en 1970 pour mettre fin à la discrimination des classes sociales et des divisions religieuses et de sexe $\mathrm{e}^{511}$, il reste des inégalités parce que les meilleures performances scolaires viennent de ceux qui fréquentent les écoles d'élite ${ }^{512}$.

Les habitudes langagières de Marie et des autres femmes de la classe inférieure qui ont recours au créole contribuent à évacuer les difficultés de leur classe inférieure. Edouard lui aussi, utilise le créole pour exprimer sa frustration et son désespoir après avoir perdu son travail : «pas ène la vie ça... ${ }^{513}$. Par contre, pour Jean-Georges Prosper, le Créole est une langue familière imprégnée d'émotivité :

\footnotetext{
505 Jean-Georges Prosper, Histoire de la littérature mauricienne de langue française, île Maurice, Editions de l'océan Indien, 1978, p. 9.

506 «Tu vas la tuer? elle sera déjà morte, pauvre bougre », Ananda Devi, Rue la Poudrière, Abidjan, Nouvelles Editions Africaines, 1988, p. 63.

507 « Sortez de là, allez ! ne venez pas faire du désordre dans ma maison, mauvais bâtards ! », Ibid., p. 35.

508 «Mallacre, c'est un bougre d'homme ! un vrai bougre!», Ibid., p. 100.

${ }^{509}$ «Mon Dieu ! Qu'est-ce que c'est ? [...] Il est déjà mort», Ibid., p. 136.

510 «Working-class (WC) speech is generally seen as one that makes great use of the vernacular and is often linked with masculinity and toughness », Susan Hughes, «Expletives of Lower Working-Class Women », Language in Society, 21, 1992, p. 291.

511 Sheila Bunwaree, «The Marginal in the Miracle: Human Capital in Mauritius », International Journal of Educational Development, 21, 2001, p. 264.

512 «The best performers come from [...] the "star' schools" », Ibid.

513 «Ce n'est pas une vie, ça », Ibid., p. 39.
} 
Au niveau de la masse [...] le parler créole est une floraison d'images saisissantes, dont l'utilisation dénote chez le peuple une manière d'intimité avec les choses de la nature ; une intimité, voire une imprégnation ${ }^{514}$.

Le créole a alors pour effet de rendre l'émotivité des personnages plus réelle et crédible.

Il paraît que les personnages utilisent le créole uniquement pour exprimer ou évacuer un sentiment, tel colère, désespoir, choc ou horreur alors que le hindi est associé à la folie comme le démontre Ganeshi, et que le français est la langue de la narration. Cette utilisation spécifique du français, du hindi et du créole dans le roman est-elle comparable à la façon dont ces langues sont utilisées à Maurice ? Selon Robert Chauderson, le créole mauricien est une langue subalterne issue de l'esclavage :

Un créole de "seconde génération" puisqu'il se développe à partir d'une situation linguistique qui ne comporte pas que le français et des langues serviles mais, outre ces langues, un créole "importé", le bourbonnais qui sert dès le début de langue de relation et est probablement appris aux premiers esclaves introduits dans le pays ${ }^{515}$.

Aussi, si le français de la narration est inévitable pour atteindre un lectorat étendu, l'emploi du créole est « pour l'auteur un moyen de rendre plus véritable le vernaculaire des personnages créoles de son roman et de marquer leur identité ${ }^{516}$. Le créole a donc pour fonction d'authentifier les personnages dans le roman et de les placer dans la classe inférieure de la population.

\section{Relations de couple dans Rue la Poudrière}

Nous proposons maintenant d'étudier les valeurs culturelles qui sous-tendent les relations de couple dans Rue la Poudrière, en examinant d'abord Marie et Edouard, les parents de la protagoniste ; puis Paule et Mallacre, son proxénète ; et Paule et Tapsy, son client devenu ami proche.

\footnotetext{
514 Jean-Georges Prosper, Histoire de la littérature mauricienne de langue française, île Maurice, Editions de l'océan Indien, 1978, p. 11.

${ }^{515}$ Robert Chauderson, Les Créoles français, Paris, Fernand Nathan, 1979, p. 51.

${ }^{516}$ Clive Gour, «Représentation du créole et thématique identitaire dans quelques œuvres littéraires d'expression française postindépendance à Maurice », mémoire, 250 pages, Ottawa, Université d'Ottawa, 2008, p. 101.
} 


\section{Marie et Edouard}

Marie et Edouard sont dépeints comme couple mais il n'est pas précisé s'ils sont mariés. Au début, le couple était heureux : «Edouard emmenait Marie au Champ de Mars pour les courses hippiques, et jouant, pour elle, un cheval gagnant [...] Edouard chantait les romances de Tino Rossi au boulevard de Marie Reine de la Paix, les soirs tièdes, effleurés d'octobre ${ }^{517}$. Mais très vite, des difficultés se font sentir et «les rancunes [et] les révoltes ${ }^{518}$ augmentent. D'abord, Edouard travaille au port pour subvenir aux besoins de la famille mais le dur labeur fait qu'il commence à boire et à dépenser l'argent du foyer aux jeux du hasard. Lorsqu'Edouard est accidenté au travail et se retrouve au chômage, il passe ses journées à la maison et les rôles commencent à s'inverser. Marie se met alors à travailler et devient chef de famille. Edouard, «triste et pleurnichard $»^{519}$, se sent inutile ${ }^{520}$ tandis que le mépris de Marie se fait évident. Cette situation les conduit à la violence physique et psychologique : «Marie et Edouard s'entrechoqu[aient] à coups de mots, à coups de pieds ${ }^{521}$. En fait, Paule rapporte que «c'était elle [Marie] qui assénait les coups à Edouard pendant son sommeil, elle tombait brusquement sur lui avec quelque chose de dur et de tranchant, elle le frappait partout, il se protégeait en hurlant ou en gémissant. Elle riait, sauvage, maléfique ${ }^{522}$. Par la suite, le roman ne donnant aucune indication sur le couple, il se peut que les deux se séparent ou continuent à vivre sous le même toit tout en étant comme des étrangers. Edouard se met à rôder alors sans but, tombant dans la pire dépravation : «Edouard, je le savais, avait fui, avait cherché d'autres voies de dégradation qui l'avaient mené jusqu'à moi. Edouard n'était rien qu'un instrument manipulé afin de voguer vers son propre échec et ma déchéance ${ }^{523}$. Déçu, inoccupé et alcoolique, Edouard se tourne vers les prostituées. Il est devenu l'exemple même du travailleur aliéné qui perd ses convictions et sa dignité. Comme l'explique Durkheim, le travailleur se tourne vers l'alcool à la fin d'une rude journée de travail pour noyer sa misère, sa fatigue et ses frustrations, puis le chômage le conduit à la dépression et au manque de confiance en

\footnotetext{
517 Ananda Devi, Rue la Poudrière, Abidjan, Nouvelles Editions Africaines, 1988, p. 36.

${ }^{518}$ Ibid., p. 7.

${ }^{519}$ Ibid., p. 39.

520 « Moi, un chien qu'on rosse à coups de pied, et puis on l'oublie, facile, laisse-le crever dans sa saleté, dans ses ordures, on crache sur lui et il fait le beau, on lui donne des restants et il doit faire dodo content », Ibid.

${ }^{521}$ Ibid., p. 15.

522 Ibid., p. 16

523 Ibid., p. 185.
} 
soi. D'après Warren Gibson, le chef de famille qui perd son emploi ressent durement son échec ${ }^{524}$.

Alcoolisme, perte d'emploi, perte de dignité, manque de confiance en soi, violence au sein du couple ; ce sont là des signes d'anomie, qui apparaît, selon Robert Merton lorsqu'il y a une rupture sociale et une insatisfaction personnelle ${ }^{525}$ qui résulte dans une cassure de l'encadrement et des liens familiaux, et dans les relations avec les autres $^{526}$. Pour John Kitsuse, l'anomie prend la forme de déviance quand l'homme s'éloigne du modèle culturel ambiant où il se doit de progresser et monter dans la hiérarchie sociale ${ }^{527}$. Merton qualifie de "retreatism", le comportement qui correspond à l'abandon majeur des pratiques culturelles qui sont généralement supposées guider la personne vers un but ${ }^{528}$. Ainsi, en vendant sa fille pour du rhum, Edouard se trouve en rupture complète avec la société. Marie, qui pratique la magie noire et la sorcellerie, et a recours au vol, est aussi en marge de la société par ses pratiques et son manque de morale. La violence et la dépravation du couple sont symptômatiques de leurs frustrations. L'échec de l'homme, le renversement des rôles et la pauvreté les ont conduits à rejeter la morale et les règles de la société.

\section{Paule et Mallacre}

Encore jeune adolescente, Paule est vendue à Mallacre par son père Edouard et le proxénète initie la jeune fille à l'univers de la prostitution. Pourtant, cet homme est, aux yeux de Paule, celui qui la sauve des griffes de ses parents, celui qui l'initie aux plaisirs du corps. Elle a beaucoup d'estime pour lui et s'abandonne à lui sans hésiter. Leur relation est surtout fondée sur leurs pulsions sexuelles, plutôt que sur l'amour.

\footnotetext{
524 «A family breadwinner who loses his job and remains unemployed for an extended period of time will surely become discouraged, a term that only begins to describe the psychological devastation that can ensue. Men especially begin to see themselves as failures not just as breadwinners, but as husbands and fathers and more generally. Marital problems often arise », Warren Gibson, « Unemployment: What is It? », A Journal for Western Man, issue cccv, 2011, p. 17.

525 «Anomie is then conceived as a breakdown in the cultural structure, occurring particularly when there is an acute disjunction between the cultural norms and goals and the socially structured capacities of members of the group to act in accord with them », Robert Merton, Social Theory \& Social Structure, New York, The Free Press, 1968, p. 216.

${ }^{526}$ «Retreatism seems to occur in response to acute anomie, involving an abrupt break in the familiar and accepted normative framework and in established social relations, », Ibid., p. 242.

527 « The behavior is deviant because it clearly represents a departure from the cultural model in which men are obliged to move onward and upward in the social hierarchy », John Kitsuse, « Societal Reaction to Deviant Behaviour: Problems of Theory and Method », Social Problems, 9, 1962, p. 248.

528 «The retreatist pattern consists of the substantial abandoning both of the once-esteemed cultural goals and institutionalized practices directed toward those goals », Robert Merton, Social Theory \& Social Structure, New York, The Free Press, 1968, p. 241.
} 
Paule voit en Mallacre un homme complètement différent de son père ${ }^{529}$. Connaissant mieux Mallacre, elle réalise qu'il y a en Edouard ce que Mallacre n'a pas et que Mallacre peut lui donner ce qu'Edouard n'a jamais pu lui donner: l'affection. La relation entre Paule et Mallacre fait naître en elle un sentiment de liberté qui l'aide à couper les ponts avec son passé familial douloureux : «[j'ai] découvert Mallacre, et [j'ai] tout oublié, l'emprise de Marie, les trahisons d'Edouard, [mon] enfance à l'odeur moisie, c'était un autre Dieu qui venait se réclamer, c'était une autre vie qu'il fallait choisir ${ }^{530}$. Elle trouve réconfort et forces auprès de Mallacre: «je me suis recroquevillée en lui, me laissant bercer, prendre, vilipender, diviniser, je ne sais plus. Tout était charnel, tout était sexuel, mais c'était la seule façon de me faire revenir à moi-même $»^{531}$.

Mais cette idylle est bientôt remise en question lorsque Paule s'aperçoit qu'elle est traitée comme les autres filles de joie et qu'elles sont toutes très dépendantes de Mallacre. Et le jour où elle voit Mallacre avec une jeune collégienne, elle réalise qu'elle a fait erreur en lui faisant confiance et qu'il n'y a, en fait, pas de différence entre cet homme et sa mère : «j'ai quitté Marie pour tomber entre les bras d'un autre donneur de mort ${ }^{532}$. Le triste sort de Ganeshi, devenue folle, lui sert aussi d'exemple. N'ayant pas le courage de se rebeller, Paule continue de rester sous l'aile de Mallacre jusqu'au jour où elle reçoit, comme client, son propre père. Dégoûtée, Paule décide enfin de quitter Mallacre et la maison close, en le critiquant ouvertement: «c'est ton jeu, ton obsession, tu aimes jongler avec les vies, entrechoquer les êtres comme des pions sur un échiquier-tu aimes jouer à être Dieu ${ }^{533}$. Mais elle se tient responsable de sa descente en enfer : « je me suis peut-être détruite moi-même $»^{534}$.

De surcrôit, Rue la Poudrière présente Mallacre comme un sauveur plutôt qu'un proxénète qui exige de plus en plus d'argent. D'après l'étude faite par Celia Williamson et Terry Cluse-Tolar le proxénète sait souvent que ses prostituées désirent faire partie d'un groupe et quitter leur vie de misère car et qu'elles font confiance à cet homme qui croit en elles, en leurs compétences, intelligence et beauté ${ }^{535}$. Nous constatons que

\footnotetext{
529 « J'ai vu un homme qui n'était pas mon père ; tout mâle qui aurait été là et qui n'était pas mon père aurait pu me cueillir de l'arbre sans peine et sans hâte », Ananda Devi, Rue la Poudrière, Abidjan, Nouvelles Editions Africaines, 1988, p. 89.

${ }^{530}$ Ibid., p. 90.

531 Ibid., p. 139.

${ }^{532}$ Ibid., p. 165.

533 Ibid., p. 181.

${ }^{534}$ Ibid., p. 189.

${ }^{535}$ Celia Williamson, Terry Cluse-Tolar, « Pimp-Controlled Prostitution », Violence Against Women, 8, 2002, p. 1081.
} 
Mallacre est l'exemple parfait du proxénète qui joue à ce jeu de séduction envers ses prostituées. De ce fait, la description de Paule correspond parfaitement à celle de la prostituée vulnérable qui se sent amadouer en contrepartie de la sécurité et de la chaleur humaine. Cette relation compliquée entre le proxénète et la prostituée qui inclut de la séduction, le contact charnel, l'échange d'argent est considérée comme illégale, ou moralement répréhensible. Ainsi, le couple Mallacre-Paule se place-t-il en marge de la société et s'avère très dysfonctionnel ${ }^{536}$. Paule se retrouve dans une situation d'anomie pour avoir voulu fuir sa famille et acquérir sa liberté.

Aussi, selon Jody Raphael et Brenda Myers-Powell, les proxénètes prétendent souvent aider les filles, car ils les font sortir de la misère et leur offre la sécurité et la nourriture ${ }^{537}$. C'est exactement ce que Mallacre promet à Paule. Ceci suggère clairement que peu importe la raison pour aller vers la prostitution, cette dernière est liée au problème de la distribution inégale des biens au sein de la société, où le pauvre ne fait que s'appauvrir et est prêt à tout pour sortir de sa situation, même si ceci signifie exploiter une autre personne dépravée. De ce fait, la conduite de Mallacre n'est moralement pas acceptable mais découle de la pauvreté ${ }^{538}$. Aussi, pour gagner de l'argent, Mallacre n'hésite pas à faire travailler ses filles et Paule, sans se soucier si elles se font avorter au prix de leur vie ou deviennent folles comme Ganeshi : « il y en avait qui étaient mortes, d'autres étaient devenues stériles ou folles. La plupart survivaient à trois ou quatre avortements sans difficultés, certaines en avaient subi jusqu'à cinq ${ }^{539}$. Doublement victime, Paule se croit en sécurité entre les bras de Mallacre mais pour ce dernier, faire travailler Paule, n'est qu'un moyen facile et sûr d'assurer sa propre survie.

\footnotetext{
536 « The extreme emphasis upon accumulation of wealth as a symbol of success in our own society militates against the completely effective control of institutionally regulated modes of acquiring a fortune. Fraud, corruption, vice, crime, in short, the entire catalogue of proscribed behaviour, becomes increasingly common when the emphasis on the culturally-induced success-goal becomes divorced from a coordinated institutional emphasis », Robert Merton, «Social Structure and Anomie », American Sociological Review, 3, 1938, p. 675- 676.

537 « To justify this control, many pimps explained that they were helping girls. They were teaching them not to give away their bodies for free. They were taking them from the gutter and feeding, housing and clothing them », Jody Raphael, Brenda Myers-Powell, «From Victims to Victimizers: Interviews with 25 Ex-Pimps in Chicago », http://newsroom.depaul.edu/pdf/family_law_center_report-final.pdf, consulté le 23 janvier 2014.

538 «Aberrant conduct, therefore, may be viewed as a symptom of dissociation between culturally defined aspirations and socially structured means », Robert Merton, "Social Structure and Anomie », American Sociological Review, 3, 1938, p. 674

${ }^{539}$ Ananda Devi, Rue la Poudrière, Abidjan, Nouvelles Editions Africaines, 1988, p. 142.
} 
Le roman suggère clairement par le biais du couple Mallacre et Paule que dans une société patriarchale les femmes pauvres et vulnérables comme Paule sont obligées à se prostituer et à tomber aux mains de Mallacre qui profite d'elles tout en prétendant les aider.

\section{Paule et Tapsy}

Tapsy, client de Paule, apparaît bien plus tard dans le roman. Sa liaison avec Paule ne dure que peu de temps mais ce couple peu ordinaire partage des moments intenses. Tous deux âgés de vingt ans, ils se sont rencontrés à la maison close et ce qui n'était qu'une rencontre sexuelle devient très vite une union de cœur, même si Paule ne l'admet pas vraiment car elle reste sous l'emprise de Mallacre. De ce fait, nous constatons qu'il s'établit un triangle amoureux et nous ne pouvons pas mentionner Paule et Tapsy sans nous référer à Mallacre.

Paule et Tapsy se sont rencontrés parce que ce dernier voulait s'initier aux plaisirs charnels : «ce Tapsy de vingt ans, qui boîtait atrocement [...] avait tenu à faire son apprentissage de l'amour afin que les autres ne se moquent pas de lui » ${ }^{540}$. Dès le début de cette rencontre, un lien spécial les lie et Paule fait une exception : «je ne lui pris pas d'argent la première fois ${ }^{541}$. Tapsy devient vite un habitué des lieux et Paule trouve en lui «une grande douceur d'approche, des caresses, des paroles rassurantes ${ }^{542}$ qu'elle apprécie, même si elle a conscience que « nos corps sont mal emboîtés, nous sommes si imparfaits, si ridicules, la rencontre de deux naufragés du destin ${ }^{543}$. De fait, Tapsy est issu de la classe pauvre, comme Paule. Il travaille au bazar de Port-Louis, où il fait le tri des fruits et légumes pourris, et vit dans la capitale, où il emmène Paule « vers sa tanière, vers son taudis, vers le plus bas des bas-fonds de Port-Louis où la vie est plus lésionnée ${ }^{544}{ }^{5}$. Toutes ces affinités avec Tapsy ne suffisent pas pour que Paule abandonne la prostitution, comme il le voudrait : « tu ne comprends pas Tapsy, je peux vendre mon corps, mais je préserve néanmoins quelque liberté, quelque fierté dans mon esprit; mais si je travaillais comme servante, j’y perdrais vraiment toute dignité, il ne me resterait rien ${ }^{545}$. Pour lui enlever tout espoir, elle n'hésite pas à utiliser des propos durs :

\footnotetext{
540 Ibid.

${ }^{541}$ Ibid., p. 142-143.

542 Ibid., p. 143.

${ }^{543}$ Ibid., p. $152-153$

544 Ibid., p. 152.

${ }^{545}$ Ibid., p. 151.
} 
Tu ne vaux rien à côté de Mallacre. Lui au moins c'est un homme, il n'a pas la patte tordue, il ne lui manque rien ; au contraire, il est plus homme que tous les autres réunis ! et qu'importe s'il nous achète s'il nous vend, si nous sommes les articles de son commerce ? ${ }^{546}$.

Même si Tapsy est doux et serein, ces qualités ne suffisent pas à Paule et quand Tapsy se dit prêt à avoir un enfant, la jeune fille trouve que Tapsy n'est pas le père idéal parce qu'il est pauvre et sans travail assuré :

et quel enfant jaillira de cela, quel infirme, quel atrophié, et que serons-nous, lorsque nous porterons le nom de père et mère, quelle Paule à la bouche mauvaise et injuriante, quel Tapsy sans travail morfondu devant son verre de rhum $^{547}$.

Paule ne veut pas reproduire ce qu'ont fait ses parents et par la suite, le couple se sépare : «j'ai laissé Tapsy ruiné, en perdition ${ }^{548}$. Ce qui a été pour Tapsy une histoire d'amour n'a été pour Paule qu'une rencontre un peu plus particulière. Dans ce cas précis, Tapsy représente le type de client vulnérable, peu attirant et seul qui recherche une relation émotive plus que sexuelle. D'après Lauren Joseph et Pamela Black, ce type de client appartiendrait à la catégorie des « hommes fragiles » ${ }^{549}$, qui réalise à un moment donné qu'il a besoin d'une connexion émotive à part les relations sexuelles avec la prostituée. En effet, Tapsy correspond bien à ce profil de client fragile qui attire la moquerie et les railleries des autres sur son lieu de travail : « les marchands et les commis alentour éclatent de rire. Il lui lancent des quolibets aux implications équivoques $\gg^{550}$. Tapsy veut aider Paule à trouver un travail plus digne et décent et même à avoir un enfant. Or, d'après Lauren Joseph et Pamela Black, la relation entre client et prostituée doit être sexuelle et sans attachment émotif quelconque, et surtout

\footnotetext{
546 Ibid., p. 150.

${ }^{547}$ Ibid., p. 153.

548 Ibid., p. 155.

549 «Men in the fragile masculinities category feel uncomfortable around women, unattractive to women and rejected by women in sexual marketplace », Lauren Joseph, Pamela Black, « Who's the Man ? Fragile Masculinities, Consumer Masculinities, and the Profiles of Sex Work Clients », Men and Masculinities, 15, 2012, p. 486.

${ }^{550}$ Ananda Devi, Rue la Poudrière, Abidjan, Nouvelles Editions Africaines, 1988, p. 145.
} 
sans désir d'enfant ${ }^{551}$. Pour la prostituée, comme pour Paule, la relation avec son client consiste à lui offrir son corps ${ }^{552}$. Cependant, Tapsy et Paule ressentent des sentiments l'un pour l'autre et tous deux expriment leur vœu d'avoir un enfant, défiant les règles de base de la prostitution. Mais, comme l'observe Charles Winick, il arrive que les prostituées répondent aux besoins émotifs de leurs clients et c'est ce qui fait que le client et la prostituée peuvent tomber amoureux l'un de l'autre ${ }^{553}$. Dès le début, Paule est à l'écoute de Tapsy: «nous bavardions beaucoup et il me racontait des histoires extraordinaires du bazar de Port-Louis, où il travaillait ${ }^{554}$. Comme le reconnaissent Joseph et Black, les prostituées peuvent jouer le rôle de psychothérapeute en s'engageant dans des dialogues ${ }^{555}$, comme le fait Paule. Tapsy est un client spécial, mais à force de voir Paule et de passer beaucoup de temps avec elle, il développe une connexion émotive avec elle et croit que ses sentiments sont réciproques ${ }^{556}$, d'autant plus que Paule, elle-même, part le retrouver sur son lieu de travail et que les deux se rencontrent hors de la maison close. Tapsy qui a cru que ses sentiments étaient partagés et vrai est brisé, bouleversé « ruiné (et) en perdition ${ }^{557}$ quand Paule a choisi de mettre fin à leur relation. Comme l'expliquent Christine Milrod et Ronald Weitzer, Tapsy a été naiff de tomber amoureux d'une prostituée et s'est mépris sur la nature de leur relation $^{558}$.

\footnotetext{
551 «Within this construct, sex, for men, should de decoupled from emotions, and men should desire, seek, and have sex for pleasure, rather than for procreation », Lauren Joseph, Pamela Black, « Who's the Man ? Fragile Masculinities, Consumer Masculinities, and the Profiles of Sex Work Clients », Men and Masculinities, 15, 2012, p. 489.

552 "Rather than offering the client her embodied self, she attempts to offer only the "raw meat" of her body and thus sexual access rather than sexual self », Belinda Carpenter, « The Prostitute and the Client: Challenging the Dualisms », Women's Studies International Forum, 21, 1998, p. 394.

${ }_{553}$ «It was usually seen as the prostitute's responding to some specific qualities of character or personality or virility of the client in a relatively direct and immediate way that might lead to love », Charles Winick, «Prostitutes' Clients' Perception of the Prostitutes and of Themselves », International Journal of Social Psychiatry, 8, 1962, p. 292.

${ }_{554}^{54}$ Ananda Devi, Rue la Poudrière, Abidjan, Nouvelles Editions Africaines, 1988, p. 143.

555 «Sex workers often describe a parallel between their work and that of a psychotherapist, describing clients who wished to be held, caressed, or even wanted only to talk during their interaction », Lauren Joseph, Pamela Black, "Who's the Man? Fragile Masculinities, Consumer Masculinities, and the Profiles of Sex Work Clients », Men and Masculinities, 15, 2012, p. 491.

556 "Clients who repeatedly see an "all-time favorite" (ATF) often develop what they perceive to be a genuine emotional connection with this person. They also believe that their feelings are experienced and reciprocated by the provider », C. Milrod, R. Weitzer, «The Intimacy Prism: Emotion Management among the Clients of Escorts », Men and Masculinities, 15, 2012, p. 450.

${ }_{557}$ Ananda Devi, Rue la Poudrière, Abidjan, Nouvelles Editions Africaines, 1988, p. 155.

558 «Some recount their gullibility and naïveté in falling in love with a provider [...] though this does not lessen the pain they feel after discovering that the relationship was not an authentic one », C Milrod, R Weitzer, «The Intimacy Prism: Emotion Management among the Clients of Escorts », Men and Masculinities, 15, 2012, p. 463.
} 
En fin de compte, les relations de couple très dysfonctionnelles décrites dans le roman sont typiques de la classe pauvre. Privés d'amour, certains personnages comme le père de Paule, tombent dans dans l'alcoolisme, ou la prostitution comme Paule. D'autres exploitent les autres pour survivre à l'image de Mallacre ou de la mère de Paule.

\section{Le rêve mauricien dans Rue la Poudrière}

Il est maintenant question d'examiner la notion de rêve mauricien au niveau des personnages. Rappelons que le rêve mauricien fait référence au fait de vouloir grimper l'échelle sociale et atteindre un niveau de vie élevé afin de jouir d'un statut social respectable, d'une bonne éducation et d'une bonne qualité de vie, même s'il faut avoir recours à la corruption ou à des moyens illégaux. Cette aspiration affecte toute la population mauricienne, qu'elle soit issue de la classe moyenne ou ouvrière, toutes ethnies confondues.

Dans Rue la Poudrière, nous constatons que Marie est l'exemple parfait de celle qui veut réaliser le rêve mauricien à tout prix car non seulement elle entreprend des emplois divers pour gagner de l'argent mais elle est aussi prête à voler ou tuer. En effet, son envie de pouvoir et sa soif d'amasser de l'argent se font sentir dans son entourage immédiat, où elle a recours à la violence pour se placer à la tête de la famille. Marie, illetrée et pauvre correspond parfaitement à l'image de la femme pauvre qui est au bas de l'échelle, comme l'affirme la directrice de Gender Links à l'île Maurice : si le chômage est répandu chez les femmes à Maurice, c'est aussi parce que beaucoup d'entre elles sont analphabètes et « 64 pour cent des femmes au chômage ne savent ni lire ni écrire ${ }^{559}$. Mais Marie, pour réaliser son rêve mauricien, défie toutes les règles sociales. Mallacre aussi est prêt à tout, même aux dépens de la vie des autres, pour améliorer ses conditions de vie.

\section{Représentation réaliste mais universelle de la classe ouvrière dans $R u e$ la Poudrière}

Ainsi, la situation économique et sociale, le mode de vie et les aspirations de ceux qui sont en bas de l'échelle sociale sont représentés de façon très réaliste dans ce roman qui met en scène les Créoles les plus démunis de la classe ouvrière qui sont souvent prêts à tout pour assurer leur survie. L'utilisation du créole comme langue vernaculaire ajoute

559 Raj Bissessur, «Chômage. Qaund la femme est victime», http://www.defimedia.info/defiquotidien/dq-societe/item/57712-chomage-quand-la-femme-est-victime.html, consulté le 13 août 2014. 
à la dimension locale du roman et montre une discrimination réelle envers les Créoles encore stigmatisés par leur antécédent d'esclavages.

\section{Le Voile de Draupadi}

Pour Le Voile de Draupadi, l'étude sera axée principalement sur la représentation des communautés hindoue, musulmane et créole et sur le registre utilisé par les personnages. Nous nous intéresserons aussi aux relations de couple entre Yashoda et son mari, entre Anjali et Dev, et entre Shyam et Margaret. Mais nous allons d'abord récapituler ce que les chercheurs ont dit à propos de la dimension sociale du roman.

Selon Bruno Cunniah et Shakuntala Boolell, qui consacrent tout un chapitre au roman et se sont concentrés sur la condition de l'Indo-Mauricienne dans la période post-indépendance, le roman de Devi est «une grande première dans la littérature mauricienne car en excluant le mâle des fruits de la relation sexuelle, la femme entend à elle seule assumer son rôle de mère ${ }^{560}$. Montrant une rupture dans le système patriarcal $^{561}$, Le Voile de Draupadi remet en question la condition de la femme hindoue qui est traditionnellement soumise à son époux et à sa belle famille. Selon Cunniah et Boolell, l'Indo-Mauricienne doit choisir de se séparer de sa famille, source de problèmes, afin de se délivrer « du poids des traditions qui la maintenaient sous la domination de l'homme ${ }^{562}$. Ashwiny Kistnareddy estime aussi que Le Voile de Draupadi dépeint la souffrance de la femme qui a le devoir de suivre le père, puis le mari, et ensuite le fils. Mais pour elle, Anjali jongle entre son milieu traditionnel hindou, où celle qui ne se conforme pas est considérée folle comme Vasanti ${ }^{563}$, et une éducation plus libérale. Elle affirme qu'Anjali accepte en fin de compte cette image libérée de la femme mauricienne en transcendant tout attachement aux ethnies qui divisent la société ${ }^{564}$. Véronique Bragard, préocupée par le «cri de femmes

\footnotetext{
${ }^{560}$ Bruno Cunniah, Shakuntala Boolell, Fonction et représentation de la Mauricienne dans le discours littéraire, Rose-Hill, Mauritius Printing Specialists LTD, 2000, p. 139.

${ }^{561}$ Ibid.

${ }^{562}$ Ibid., p. 143.

${ }^{563}$ Ashwiny Kistnareddy, «Interrogating Identity: Psychological Dislocations in Ananda Devi's Novels », Dalhousie French Studies, 94, Spring 2011, p. 31.

564 «Anjali has been able to transcend the barriers between herself and the island, which her grandfather had erected through his madness at his loss of identity as an Indian [...] Anjali chooses her present identity as a Mauritian woman, which is not multiple and plural but devoid of ethnic attachment », Ibid., p. 32.
} 
maudites » ${ }^{565}$ suggère également que Devi dépeint des femmes qui sont « rejetées par leurs communautés et l'autorité patriarcale qui les domine ${ }^{566}$. Elle établit un parallèle entre la traversée de l'océan par les travailleurs indiens et la traversée dans la souffrance par ces femmes, qui dans ce processus, doivent faire face au rejet et à l'abandon ${ }^{567}$. Elle conclut que, même si Devi présente des personnages femmes qui font face à des situations sociales opprimantes et difficiles, «l'écrivaine mauricienne nous invite à admirer la force qui se dégage de l'être humain, bien décidé à donner sens à son existence $»^{568}$.

Pour sa part, Srilata Ravi étudie, dans Le Voile de Draupadi, le rapport entre le mythe hindou et la formation de l'identité hindoue à Maurice aussi bien que le rapport entre les pratiques religieuses et la santé. Elle considère aussi les conventions sociales et religieuses et comment la femme hindoue doit posséder des qualités calquées sur Sita et Draupadi, les héroïnes des épopées indiennes Ramayana et Mahabharata, respectivement. D'après elle, Le Voile de Draupadi est une représentation réaliste des complexités de la société hindoue mauricienne ${ }^{569}$. Selon Ravi, Devi dénonce aussi les dogmes hindous qui participent de la société patriarcale hindoue et prône une diversité de la femme mauricienne hindoue ${ }^{570}$. Faisant référence au jeûne d'Anjali, Ravi avance que la jeune femme s'est tellement privée de nourriture qu'elle tombe dans l'anorexie et qu'elle devient hystérique, songant même à se laisser mourir lors de la marche sur le $\mathrm{feu}^{571}$. Njeri Githire, elle, observe que l'alimentation peut être source de tension et de conflit mais aussi de force ${ }^{572}$. Se concentrant sur la raison de la non-alimentation dans Le Voile de Draupadi dans le contexte religieux, culturel et social d'Anjali, elle est d'avis que, dans ce roman, le refus de s'alimenter aide à défier et subvertir le système

\footnotetext{
${ }^{565}$ Véronique Bragard, «Cris des femmes maudites, brûlures du silence, la symbolique des éléments fondamentaux dans l'œuvre d'Ananda Devi », Notre librairie, 142, 2000, p. 66. traversée transocéanique de leurs ancêtres indiens venus travailler à Maurice, il révèle également la traversée des interdits qu'elles doivent entreprendre pour se délivrer des carcans, des rôles et du poids des traditions et des croyances populaires qui les cloisonnent. Cette transgression a cependant un prix : une grande aliénation et une profonde solitude », Ibid.

568 Ibid., p. 73.

569 Srilata Ravi, «Religion, Health and Hindu Woman in Mauritius: Ananda Devi's Le Voile de Draupadi », Kunapipi, Journal of Postcolonial Writing, 28, 2006, p. 69.

${ }^{570}$ Ibid., p. 75.

571 «The ritual of fasting depletes Anjali physically and she verges on hysteria. She purposefully takes the fast to such an extreme point in the hope that her weakened, anorexic body will not survive after the fire sacrifice », Ibid., 73 .

$572 «[\ldots]$ the portrayal of food as a site of tension and conflict, but also as a potent force in forging selfdefinition », Njeri Githire, «The Semiotics of (Not) Eating: Fasting, Anorexia, and Hunger Strike in Ananda Devi's Le Voile de Draupadi », Nottingham French Studies, 48, 1, 2009, p. 82.
} 
social qui est au pouvoir ${ }^{573}$. Le jeûne n'affecte d'ailleurs pas seulement Anjali mais aussi son oncle qui finit par sombrer dans la folie pour avoir trop jeûné ${ }^{574}$. Si Githire montre les effets néfastes d'un excès de jeûne, elle discute aussi de la source et de l'importance rattachée au jeûne dans l'hindouisme. Régi par le patriarcat, le rituel du jeûne, surtout par la femme, est fondamental pour atteindre la piété ${ }^{575}$. Kistnareddy ${ }^{576}$ fait ressortir l'importance du feu dans la culture hindoue en montrant que si Vasanti s'est immolée, Anjali ressent l'énergie de ce feu brûler en elle, tout comme Draupadi, l'héroïne du Mahabharata, qui était née du feu ${ }^{577}$. Kistnareddy conclut qu'à travers la souffrance de ses personnages féminins, Devi suggère que chacun doit simplement s'accepter tel qu'il est sans s'attacher aux autres pour se définir ${ }^{578}$.

Srilata Ravi $^{579}$ mentionne le voile qui a une place importante dans la tradition indienne puisque le sari de la femme hindoue symbolise la chasteté et la pureté.

Tous ces travaux consacrés au Voile de Draupadi sont axés sur la souffrance de l'Indo-Mauricienne soumise à des traditions indiennes pesantes. Si la plupart de ces articles mettent en avant l'oppression de la femme hindoue à Maurice, nul ne s'intéresse à la disparité entre Hindous et Créoles, ni au registre de langue qui catégorise les personnages, ni aux relations de couples. Dans ce chapitre, nous analyserons, pour en évaluer le degré de réalisme, le milieu social, les conditions de vie des personnages, et les rapports au sein des couples formés par les parents d'Anjali, et au sein du couple Dev et Anjali et au sein du couple Shyam et Margaret.

\section{Représentation des ethnies dans Le Voile de Draupadi}

Le Voile de Draupadi dresse surtout le portrait de la famille hindoue à travers Anjali mais met aussi en scène des personnages issus des communautés musulmane et créole. Fatmah et Faisal sont des amis musulmans d'Anjali et de Dev, alors que

\footnotetext{
573 «I will endeavor to read (not-eating as a form of resilience which is interpolated into systems of power in order to subvert them », Ibid., p. 83.

574 «The memory of an uncle who fell apart, slipping into madness following extensive fasting and sacrifice and ascetic hardships is also deeply ingrained in Anjali's mind », Ibid., p. 85.

575 «The entanglement between food, sex and patriarchal control of women's bodies accounts for the reason why abstention from food as a practice necessary in the pursuit of holiness has been constructed through particular histories and practices to be more salient in the piety of women than in that of men ", Ibid., p. 87.

${ }^{576}$ Ashwiny Kistnareddy, « Interrogating Identity: Psychological Dislocations in Ananda Devi's Novels », Dalhousie French Studies, 94, Spring 2011, p. 27-38.

577 « The symbolism of fire is very important here as Vasanti died by fire, and Anjali feels fire burning inside her just as Draupadi was born out of fire », Ibid., p. 31.

578 «If the question of identity brings about such suffering as has been analyzed in this article then, Devi suggests, we should not persist in our quest but should simply be », Ibid., p. 36.

579 Srilata Ravi, «Religion, Health and Hindu Woman in Mauritius: Ananda Devi's Le Voile de Draupadi », Kunapipi, Journal of Postcolonial Writing, 28, 2006, p. 67- 78.
} 
Marlène et Matante Sec sont créoles, et respectivement servante ou sorcière. Il faudra donc préciser l'appartenance ethnique des personnages, analyser la représentation de ces ethnies, et les rapports entre ces communautés afin d'examiner la question de la disparité entre ces groupes sociaux.

\section{La communauté hindoue}

Anjali est sans conteste issue d'une famille hindoue ${ }^{580}$, mais de foi tamoule et de caste supérieure. Sa mère et son oncle conversent en « un tamoul très littéraire [qu'elle] ne comprenai[t] pratiquement pas ${ }^{581}$. Les noms des personnages aussi sont typiquement hindous. Ainsi, «Anjali signifie prière ${ }^{582}$, Dev fait référence au Dieu solaire $^{583}$, Vasanti dénote le printemps ${ }^{584}$, Shyam serait un pseudonyme pour le dieu Krishna, et Yashoda est le nom de la mère nourricière de Krishna dans le Mahabharata. Par ailleurs, Anjali se rappelle que son mariage est célébré à l'indienne en : « faisant le tour du feu ${ }^{585}$ et en portant « un sari blanc et or ${ }^{586}$. Le roman précise également l'origine supérieure de la famille : «tous les signes de la caste sont là : la couleur de la peau [...] la longueur des doigts effilés, la main grande mais étroite, la peau presque transparente ${ }^{587}$ et il est important, pour cette famille, de se marier de génération en génération, au sein de la caste ${ }^{588}$. Le fait qu'Anjali va au temple pour prier, jeûner et accomplir la marche sur le feu sont la confirmation qu'elle fait partie de la communauté hindoue.

Les descriptions des personnages hindous sont très flatteuses. Ainsi, la mère d'Anjali est décrite comme ayant un « grand rire clair » ${ }^{589}$ et l'héroïne se rappelle que sa «mère est belle. Elle est belle parce qu'il y a en elle une élégance innée » ${ }^{590}$. Quant à Vasanti, qui a les cheveux longs ${ }^{591}$, elle «était belle, pratiquement sans défauts physiques ${ }^{592}$, « une fée ${ }^{593}$. La belle-mère d'Anjali a une «apparence étonnamment

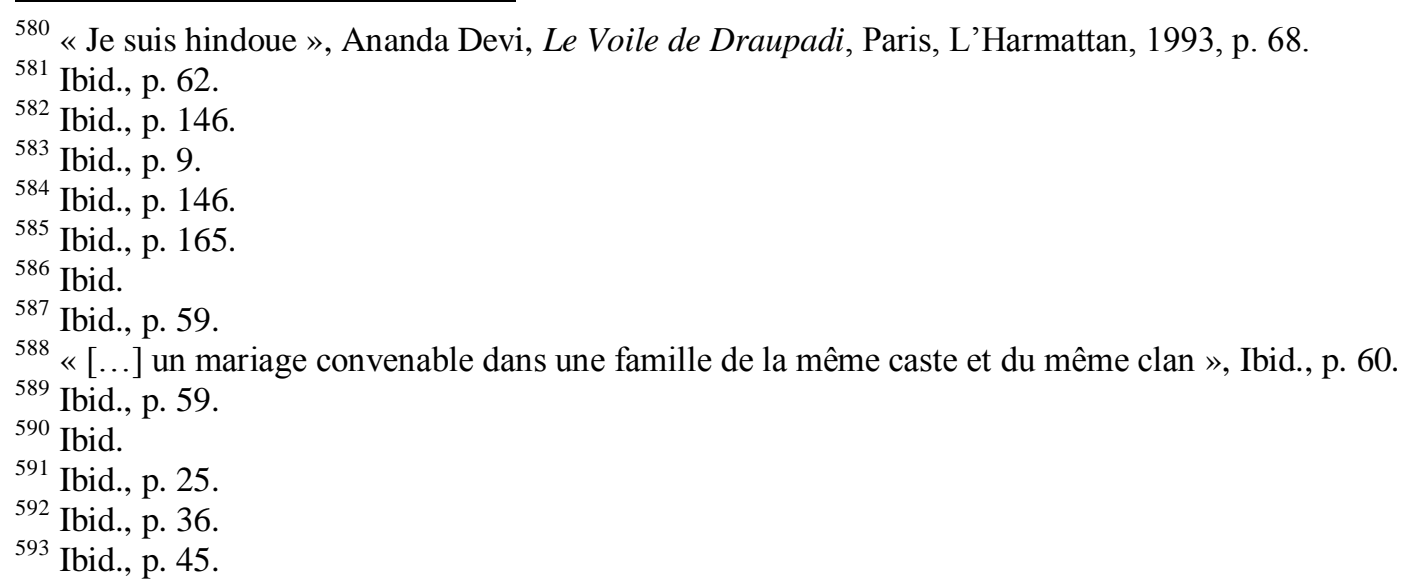


jeune ${ }^{594}$. Les hommes aussi ont droit à des descriptions flatteuses. Shyam a, comme son père, « les yeux brun-clair, que le soleil rend presque dorés, toujours allumés d'une douceur toute simple, le sourire presque permanent sur ses lèvres minces ${ }^{595}$.

Ces traits physiques sont traditionnellement associés à la caste supérieure des Hindous. Aussi, le grand-père d'Anjali est arrivé à l'île Maurice avec la notion des castes héritée du système social indien qui divise les Hindous en différentes castes. Le vieil homme perpétue en particulier la tradtidion du mariage arrangé : «surtout le respect de cette tradition [du mariage arrangé] était primordial. Rien ne devait altérer ce sang préservé depuis des siècles, ni la symbolique pureté de la lignée » ${ }^{596}$. Étant de caste supérieure, le grand-père entend préserver la tradition de marier ses enfants dans le même clan; exactement comme le constate le rapport de la Truth and Justice Commission $^{597}$. C'est aussi le principe que suit Yashoda lorsqu'elle marie sa fille Anjali à Dev. Pour cette union, les deux familles s'assurent que la situation sociale et financière est maintenue pour les deux familles : «rien que cette "réunion" de part et d'autre, les deux familles assises, s'observant, se regardant, se "mutualisant" et énumérant mentalement les avantages du mariage $»{ }^{598}$. Cette tradition indienne fortement ancrée à l'île Maurice au sein des Hindous est représentée par la famille d'Anjali dans le roman.

De même, la ségrégation basée sur la couleur de la peau a perduré dans la société indienne à Maurice. La croyance du grand-père d'Anjali que le teint clair est égal à la beauté trouve ainsi sa source dans la colonisation de l'Inde quand les riches colons blancs anglais étaient considérés comme supérieurs ${ }^{599}$ aux Indiens de teint foncé. Donc, une peau blanche représente non seulement la beauté mais aussi la suprématie économique. Cette question de couleur de la peau est encore bien ancrée, même de nos jours dans la société mauricienne où l'on associe toujours la peau claire à

\footnotetext{
${ }^{594}$ Ibid., p. 92.

595 Ibid., p. 142.

${ }^{596}$ Ibid., p. 60.

597 «As the Social Stratification project of the TJC (2011) confirms, when the Indian indentured labourers arrived in Mauritius, they did come to the island with unchanged ideas about and experiences of caste », Truth \& Justice Commission, « Report of the Truth and Justice Commission. Volume 3. Contemporary History, Culture and Society. Research Reports, Technical Studies and Surveys », http://pmo.gov.mu/English/Documents/TJC_Vol3.pdf, consulté le 13 mars 2014, p. 548.

${ }_{598}$ Ananda Devi, Le Voile de Draupadi, Paris, L'Harmattan, 1993, p. 20.

599 «The whitened face of Asia is [...] demanded from a colonial system that has extracted itself politically from the scene of colonial invasion », P. Goon, A. Craven, «Whose debt?: Globalisation and Whitefacing in Asia », http://intersections.anu.edu.au/issue9/gooncraven.html, Intersections: Gender, History and Culture in the Asian Context, 9, 2003.
} 
la caste supérieure et le teint foncé à la caste inférieure ${ }^{600}$, attitude colportée par le grand-père d'Anjali.

Les mariages arrangés qui se font majoritairement au sein de la même caste comme dans Le Voile de Draupadi ${ }^{601}$ perdurent-ils toujours à l'île Maurice ? Il est aussi intéressant de se demander si les Mauriciens jugent toujours les gens sur la couleur de leur peau. D'après la Truth and Justice Commission, les règles qui définissent et divisent les différentes catégories de caste à l'île Maurice n'ont jamais été très claires, et ceci dès le début de la venue des travailleurs engagés ${ }^{602}$. Mais les Hindous ont conservé les divisions de caste de l'Inde comme telles et de fait, ceux de la haute caste sont généralement prêtres ${ }^{603}$. Cette commission d'enquête établit aussi que l'appartenance à la caste est généralement prise en considération lorsque deux familles hindoues s'unissent dans les liens du mariage ${ }^{604}$.

\section{La communauté musulmane}

Le Voile de Draupadi dépeint des personnages issus de la communauté musulmane par l'entremise de Faisal et Fatmah, cousine de ce premier ${ }^{605}$, et de toute la famille, qui habite le quartier musulman de Port-Louis. Fatmah, fille célibataire de quarante ans, est l'amie d'Anjali alors que Faisal, client de Dev, est un riche vendeur de drogue. Cette famille étendue habite depuis un demi-siècle dans un quartier où « il s'y emboîtait plusieurs maisons, les unes reliées aux autres par un couloir ou une varangue vitrée. Chaque maison était occupée par un fils ou un neveu du patriarche ${ }^{606}$. Dans cette famille patriarcale, Fatmah n'a aucun statut, car elle n'a ni père, ni mari et «puisque Fatmah ne pouvait pas vivre seule (la réputation de la famille serait mise en

\footnotetext{
${ }^{600}$ Shilpi Bhattacharya, «The Desire for Whiteness: Can Law and Economics Explain it? », Columbia Journal of Race and Law, 2, 2011, p. 125.

${ }^{601}$ «Rien que cette formelle "réunion" [d'Anjali et de Dev], de part et d'autre, les deux familles assises, s'observant, se regardant, se "mutualisant" et énumérant mentalement les avantages du marriage », «Elle [Yashoda] a été une fille à la maison, attendant d'être mariée, et à dix-sept ans, elle a fait un mariage convenable dans une famille de la même caste et du même clan ", Ananda Devi, Le Voile de Draupadi, Paris, L'Harmattan, 1993, p. $20^{2}, 60$.

602 « In Mauritius, the lines were never so clear-cut, nor were they, in the beginning, at the Emigration or Immigration depot, on board the ship », Truth \& Justice Commission, « Report of the Truth and Justice Commission. Volume 1 », http://www.usip.org/sites/default/files/ROL/TJC_Vol1.pdf, consulté le 3 mars 2013, p. 290.

${ }^{603}$ «Mauritians have retained some of the stereotypical occupational specializations which categorized Hindus in India, such as the fact that Chamars were leather workers, or Ahirs, were cattle-rearers and Brahmins were priests », Ibid., p. 293.

${ }^{604}$ Ibid., p. 292.

${ }^{605}$ « Tu [Faisal] as une cousine, Fatmah, qui habite un réduit dans ton propre sous-sol pace que ta mère, cette vieille matriarche à moitié folle, la déteste», Ananda Devi, Le Voile de Draupadi, Paris, L'Harmattan, 1993, p. 54.

${ }^{606}$ Ibid., p. 102.
} 
question), Faisal lui aménage au sous-sol une espèce d'appartement sommaire, avec une latrine couverte et une douche à l'extérieur, derrière un arbre ${ }^{607}$. Cette situation précaire ne décourage pas Fatmah, très croyante et pratiquante, «la tête voilée, agenouillée sur le tapis de prière ${ }^{608}$, elle continue de prier, même pour la guérison de Wynn, avec des «Inch’ Allah » ${ }^{609}$. Comparée à Vasanti par Anjali, Fatmah est plus qu'une amie : «Fatmah avait été, d'une certaine façon, un de [ses] tuteurs » ${ }^{610}$ qui la guide et l'empêche de baisser les bras.

Tout comme les personnages hindous, les Musulmans sont décrits dans le roman de manière positive. Ainsi, Fatmah a toujours « un sourire permanent et empli de cette douceur illuminée ${ }^{611}$, avec des «tresses épaisses qui lui descendaient aux mollets ${ }^{612}$.

\section{La communauté créole}

Si, dans Le Voile de Draupadi, les Hindous sont riches et respectés et les Musulmans sont fortunés et puissants, les Créoles de leur côté sont pauvres, au service des Hindous, ou coupables de sorcellerie. Contrairement à la description de Shyam qui est souriant et beau avec ses yeux brun-clair, sa concubine, Margaret, est décrite comme étant « une grosse fille sombre, aux cheveux crêpelés, à la bouche indécise et morne, et si épaisse à côté de Shyam » ${ }^{613}$. Alors que la famille de Shyam est bien éduquée et respectée, celle de Margaret provient « de la plus basse couche sociale du pays ${ }^{614}$ et les femmes de son entourage sont «miséreuses [...] criardes [et] crues ${ }^{615}$.

Marlène, autre personnage créole et la servante d'Anjali, est peu attrayante : « une grosse femme dont la jeunesse ne se révélait qu'au son de sa voix [...] elle avait une tête carrée aux cheveux crépus et ras, et portait des vêtements toujours trop étroits qui avaient l'air de vouloir éclater au moindre effort sur ses chairs noires ${ }^{616}$. Matante Sec, une sorcière et guérisseuse créole, elle, «portait des vêtements disparates : un veston d'homme trop grand, une sorte de pantalon chinois flottant, de grosses chaussures au bout béant comme une gueule de poisson. Une immense chevelure

\footnotetext{
${ }^{607}$ Ibid., p. 104

${ }^{608}$ Ibid., p. 103.

${ }^{609}$ Ibid., p. 105.

${ }^{610}$ Ibid., p. 100.

${ }^{611}$ Ibid.

612 Ibid., p. 103.

${ }^{613}$ Ibid., p. 19.

614 Ibid., p. 122.

615 Ibid.

${ }^{616}$ Ibid., p. 68.
} 
crêpelée se répandait autour de sa tête, injectée de gris et de blancs » ${ }^{617}$ et qui avait « une très forte odeur, presque masculine, de sueur, de vieillesse, de rhum, un véritable relent de pauvreté et de décrépitude, vertigineux ${ }^{618}$.

Les Créoles décrits dans le roman font partie des pauvres mal habillés, laids et sans éducation, qui travaillent pour les autres. Par leurs traits physiques et leur statut social subalterne, ils s'opposent aux communautés hindoue et musulmane riches.

\section{Les disparités raciales et discriminatoires entre les communautés hindoue et créole dans Le Voile de Draupadi}

Il est évident que ce roman évoque une grande disparité entre les personnages hindous, musulmans et créoles. Si les Hindous sont toujours présentés comme beaux, riches et distingués et les Musulmans comme riches, les Créoles sont très pauvres et mal habillés, émettent des odeurs corporelles déplaisantes, et ne sont jamais beaux à voir. Ainsi, la famille riche d'Anjali, qui comprend de hauts cadres, peut se permettre d'avoir deux servantes, l'une hindoue et l'autre créole. Si la servante hindoue «nettoyait la maison et préparait quelquefois le repas ${ }^{619}$, la bonne créole « faisait la lessive et le repassage trois fois par semaine ${ }^{620}$. C'est dire que la servante hindoue, chargée des tâches ménagères à l'intérieur de la maison, peut avoir accès à la cuisine pour préparer la nourriture alors que la bonne créole, responsable du lavage et repassage, a un accès limité à l'intérieur de la maison et à la cuisine. Ressentant cette disparité et ressentant peut-être cette hiérarchie sociale, la bonne créole se rebelle en maintenant « une rivalité perpétuelle et sans cesse grandissante avec la servante hindoue $»^{621}$.

Cette représentation des trois ethnies reflète-t-elle une réalité ou est-elle une stéréotypisation raciste de la société mauricienne ? L'apparence physique et surtout la couleur de la peau de ces personnages sont souvent source de discordances entre les groupes ethniques présents dans ce roman. Ce sont justement ces discordances que nous analyserons afin de voir si elles émergent des cas stéréotypés de la société mauricienne.

\footnotetext{
${ }^{617}$ Ibid., p. 69.

${ }^{618}$ Ibid.

${ }^{619}$ Ibid., p. 68.

${ }^{620}$ Ibid.

621 Ibid.
} 
Le rejet des Créoles par les Indiens existe depuis que les travailleurs engagés, confrontés aux esclaves noirs, les ont considérés comme inférieurs, à cause de leurs traits physiques, de la couleur de la peau noire et du fait qu'ils travaillaient la terre. Pour l'Indien, celui qui a la peau noire est associé aux durs labeurs sous le soleil et celui qui a la peau blanche est issu d'une famille riche, ou a un niveau d'éducation élevé $^{622}$. Donc pour le travailleur engagé, l'esclave noir représente celui qu'il doit rejeter et ignorer car il juge que ce travailleur noir lui est inférieur puisqu'il ne correspond pas à sa définition de beauté; celle d'être grand et mince et de teint claire $^{623}$. C'est ainsi que les descendants d'esclaves à Maurice se retrouvent doublement esclave ; de leur maître blanc et des préjugés des Hindous. Pourtant, Anjali reconnaît finalement que malgré son apparence physique, Margaret est une personne sympathique. En reconnaissant la bonté et la douceur de celle-ci, Anjali symbolise peut-être une jeune génération hindoue mauricienne qui accepterait que le Créole fasse partie de son cercle familial :

Si au début nous avons tous été choqués de son apparence, du fait qu'elle provenait de la plus basse couche sociale du pays, n'était ni de notre race ni de notre religion, j'ai dû reconnaître plus tard que Margaret avait extrait de ses origines miséreuses quelque chose de beau [...] son physique épais était vite contredit par la douceur de sa voix et de son regard ${ }^{624}$.

Si à l'époque du grand-père d'Anjali, pour être joli il fallait être de teint très clair avec les yeux bleus, désormais pour l'Indo-Mauricienne, cette définition trouve sa source dans les films et séries télévisées indiennes où l'héroïne, grande, mince, bien maquillée et portant les vêtements de dernier cri, incarne la beauté ${ }^{625}$. De même, la jeune génération mauricienne commence peu à peu à changer ses critères de beauté

\footnotetext{
${ }^{622}$ «Dark skin is associated with labour and field work in the sun and fair skin with wealth, aristocracy and higher education », K. Islam, H. Ahmed, E. Karim, A. Amin, « Fair Factor. The Whiter the Better», http://archive.thedailystar.net/magazine/2006/05/02/cover.htm, consulté le 14 février 2014.

${ }_{623}$ «What is considered beautiful is often very clear. Beauty is fair, tall and slim », Susan Runkle, « The Beauty Obsession. International Standards for Fair and Lovely », http://www.manushiindia.org/pdfs_issues/PDF\%20145/145\%2011-16.pdf, consulté le 10 février 2014.

${ }_{624}$ Ananda Devi, Le Voile de Draupadi, Paris, L'Harmattan, 1993, p. 122.

625 «Indo-Mauritian young women are strongly attracted to the make-up, the dress and the body figures of the actresses », Angela Ramsoondur-Mungur, «Female Indo-Mauritian Negotiation of Beauty and Bollywood TV Serials », https://www.academia.edu/993399/Female_IndoMauritian_negotiation of beauty and Bollywood TV serials draft_paper, consulté le 10 février 2014, p. 5
} 
depuis que les séries télévisées de Bollywood montre que la femme indienne peut aussi être belle et élégante sans être blanche ${ }^{626}$. Ainsi, nous notons un retour aux sources pour les Indo-Mauriciens pour qui être jolie veut désormais dire ressembler aux actrices indiennes ${ }^{627}$ et non pas ressembler au colon à la peau blanche et aux yeux bleus.

A titre indicatif, nous proposons de nous baser sur les chiffres récents pour analyser la situation des personnages du roman. Ainsi, d'après la Truth and Justice Commission, les jeunes Créoles mauriciens sont d'avis qu'il existe encore à l'île Maurice une préférence pour la peau blanche qui limite leurs droits dans certains secteurs $^{628}$. En plus, la commission signale que les jeunes femmes créoles mauriciennes sont les plus ciblées et qu'il leur est très difficile de trouver un travail décent et bien rémunéré $^{629}$. La commission d'enquête ajoute que même si une personne peut être ami avec une personne d'une autre ethnie, il n'est pas pour autant permis de se marier entre ethnies différentes ${ }^{630}$. La commission est d'avis que les Indo-Mauriciens sont encore très rattachés à des traditions et cultures indiennes et conclut que bien que la société mauricienne montre une certaine ouverture, il existe toujours des valeurs racistes à l'île Maurice $^{631}$, comme le suggère Le Voile de Draupadi à travers l'union de Shyam et de Margaret, où les parents du jeune homme, pas prêts à accepter cette relation, réagissent pour montrer leur résistance et déception. Ainsi, Yashoda «pleurait, pleurait, jour et nuit (et) s'était mise à boire secrètement ${ }^{632}$ alors que son mari « se réfugiait dans sa chambre, silencieusement blessé au cœur par la trahison du fils $»^{633}$.

\footnotetext{
626 « There is no more this understanding that to be blonde or brunette with blue or grey or green eyes, that is, to be white means to be beautiful. The Indian woman [...] and Indian actresses [...] are seen as making part of the package "Beautiful" », Ibid., p. 6.

627 « Being beautiful means to be looking alike to the Indian actresses. Being beautiful for the IndoMauritian women is to mimic the beautiful Indian Women », Ibid., p. 8.

${ }^{628}$ "The youths are frustrated by the continuation of a "pigmentocracy" (social hierarchy based on the privileging of whiteness) in Mauritius [...] as significant minority, Creoles could never obtain advantages or even basic rights in Mauritian society », Truth \& Justice Commission, « Report of the Truth and Justice Commission. Volume $1 »$, http://www.usip.org/sites/default/files/ROL/TJC_Vol1.pdf, consulté le 3 mars 2013, p. 284.

${ }^{629}$ «The Commission finds that black-skinned, young Creole or slave descendant women in Mauritius experience the worst form of racism [...] they find it difficult to obtain decently-paid work », Ibid., p. 287.

${ }^{630}$ «Interestingly, while a young person may not necessarily be denied friendship with a person of a different religion or "race", the moment when marriage is being considered, then the young person is pressurised into finding someone of his or her own 'kind' », Ibid., p. 285.

631 «There appears to be social cohesion in Mauritius, but this is at the surface-level only. At a deeperlevel, Mauritius is an openly racist and bigoted country », Ibid., p. 288.

${ }^{632}$ Ananda Devi, Le Voile de Draupadi, Paris, L'Harmattan, 1993, p. 122.

633 Ibid.
} 


\section{La représentation des classes sociales dans Le Voile de Draupadi}

Dans Le Voile de Draupadi Dev est avocat et Shyam est cadre supérieur dans une entreprise privée. Pour la famille d'Anjali, d'origine hindoue, les hommes occupent de bons postes tandis que les femmes restent au foyer pour assurer la continuité de la lignée, bien élever et éduquer les enfants. Outre la famille hindoue aisée, ce roman dépeint aussi des personnages créoles féminins, employés par les familles hindoues.

\section{La classe aisée dans Le Voile de Draupadi}

Le roman ne livre pas de descriptions détaillées de la maison de Dev et Anjali mais Anjali fait allusion à l'immensité de leur propriété. Par exemple, lorsque Wynn est malade, Anjali passe la plupart de son temps dans la chambre de l'enfant et c'est pour cela que «les autres pièces » ${ }^{634}$ lui semblent «inconnues, froides, vêtues d'un vague délabrement ${ }^{635}$. Elle trouve refuge dans le jardin de sa maison où «l'air est encore doux » ${ }^{636}$ et où « la balançoire de Wynn » ${ }^{637}$ lui rappelle des moments heureux passés avec son fils. Nous savons aussi que pour maintenir la propriété, le couple emploie deux servantes. Ces descriptions suffisent pour suggèrer que le couple habite dans une grande maison avec un jardin. De plus, il a les moyens d'avoir une voiture ${ }^{638}$. Leurs vêtements sont aussi preuve de richesse. Ainsi, à la fête de Faisal, Anjali est : «vêtue de [son] pesant sari de soie brochée, le khol aux yeux, les bijoux aux oreilles, au cou et au poignet $»^{639}$. Le roman ne donne pas de détails sur le métier du père d'Anjali mais nous savons que sa mère Yashoda a toujours été femme au foyer et qu'ils habitent dans une «grande maison coloniale ${ }^{640}$, avec un grand jardin et un kiosque qui sont présentés de façon très détaillée dans le roman :

[Cette maison] était démesurément solide [...] les bardeaux s'accrochaient encore fermement aux combles gracieux, couronnés de paratonnerres blancs, la véranda vitrée au perron de pierres taillées livraient au regard des solives épaisses et patinées par l'âge. Une tête de cerf aux grands bois, au regard langoureux, pendait encore au mur

\footnotetext{
${ }^{634}$ Ibid., p. 7.

${ }^{635}$ Ibid.

${ }^{636}$ Ibid., p. 31.

${ }^{637}$ Ibid., p. 32.

${ }^{638}$ « Dev m'a prise par le bras, le regard dur et sec, et m'entraîne vers la voiture », Ibid., p. 55.

${ }^{639}$ Ibid., p. 52.

${ }^{640}$ Ibid., p. 120.
} 
de la véranda $[\ldots]$ le grand salon au plafond blanc, frangé de moulures, arborait une ancienne tapisserie peinte à la main $^{641}$.

D'autre part, la famille de Dev aussi est riche puisque selon Anjali une des tantes de Dev «s'était affublée d'une multitude de bijoux en or ${ }^{642}$.

Leur ami Faisal qui est Musulman est un homme riche. Ce dernier, lors d'une fête organisée dans sa maison au «goût extrêmement coûteux » ${ }^{643}$, a en effet, servi à ses invités du « caviar-champagne-saumon fumé » ${ }^{644}$, un « merveilleux vin blanc ${ }^{645}$ et d'autres «mets délicats et chers ${ }^{646}$. Les invités, visiblement riches, étaient habillés de vêtements des hauts couturiers comme, «Pierre Cardin, Christian Dior, Cartier ${ }^{647}$. Aussi, le roman dépeint-il une classe riche associée à la caste supérieure hindoue et à certains Musulmans.

\section{La classe inférieure dans Le Voile de Draupadi}

Le Voile de Draupadi dépeint aussi des personnages de la classe inférieure, tels Marlène, la servante créole d'Anjali, Matante Sec, la sorcière créole, et Margaret, la concubine créole de Shyam. Si nous n'avons pas de détails sur le lieu où demeure Marlène, nous savons que Matante Sec habite « dans une espèce de bouge envahie de bêtes empaillées qu'elle avait elle-même capturés et tuées » ${ }^{648}$, alors que Margaret «provenait de la plus basse couche sociale du pays » ${ }^{649}$, et avait des «origines miséreuses ${ }^{650}$. Il est intéressant de noter que tous ces personnages sont des femmes créoles et pauvres. En présentant ces femmes comme personnages démunis et faibles, Devi montre avec réalisme les conditions sociales des femmes créoles à l'île Maurice où elles sont doublement victimes ; comme Créoles et comme pauvres.

\footnotetext{
${ }^{641}$ Ibid., p. 123.

${ }^{642}$ Ibid., p. 94.

${ }^{643}$ Ibid., p. 53.

${ }^{644}$ Ibid., p. 55

${ }^{645}$ Ibid., p. 52.

${ }^{646}$ Ibid., p. 54.

${ }^{647}$ Ibid., p. 55.

${ }^{648}$ Ibid., p. 69

${ }^{649}$ Ibid., p. 122

${ }^{650}$ Ibid.
} 
C'est ainsi qu'à travers ces deux classes sociales opposées, Devi brosse « une peinture réaliste des milieux avec l'exactitude de l'analyse sociale » ${ }^{651}$. Cette représentation des catégories sociales du roman calque bien une réalité sociale mauricienne qui perdure toujours.

\section{Le registre de langue utilisé dans Le Voile de Draupadi}

Le registre de langue employé par la famille aisée d'Anjali démontre leur appartenance à l'élite. En effet, Anjali utilise toujours un français standard pour s'adresser à son entourage mais Dev et le médecin traitant de Wynn, le docteur Pradhan, communiquent en anglais : " you see, my dear, he'll have much better care in the clinic, we can do the necessary tests immediately and put him on the drip. If it is meningitis, it is probably quite benign, of course... ${ }^{652}$. Dev s'adresse alors en anglais à Anjali : «for god's sake ! ${ }^{653}$. À la fin du roman, le médecin et Dev parlent encore en anglais: "you know, meningitis is meningitis, in its acute form there is nothing much you can do ${ }^{654}$, « give him an overdose then ! [...] give him an overdose and let it end $! »{ }^{655}$.

Par contre, les autres personnages font appel soit au créole, soit au bhojpuri lorsqu'ils communiquent. Il est à noter que ces personnages ne sont pas nécessairement issus de la classe défavorisée, ni ne sont nécessairement des Créoles. Par exemple, le prêtre tamoul qui s'adresse à Anjali a recours au créole ${ }^{656}$ et une des invitées, apparemment riche, s'adresse aussi en créole à Anjali à la fête de Faisal ${ }^{657}$.

Ces échanges en différentes langues sont-ils représentatifs de la situation linguistique à l'île Maurice ? D'après Issa Asgarally, linguiste mauricien, les notions de langue et de colonialisme «sont intimement liées » ${ }^{658}$. C'est pourquoi l'anglais, héritage des colons anglais, est actuellement la langue officielle et la «langue du pouvoir ${ }^{659}$, alors que le français est resté la langue du prestige ${ }^{660}$. En effet, utilisée au

\footnotetext{
${ }^{651}$ Elaheh Salehi Rizi, «Les jeux entre la réalité et la fiction dans le roman : On s’y fera de Zoyâ Pirzâd », http://revel.unice.fr/symposia/actel/index.html?id=364, consulté le 20 août 2014.

${ }^{652}$ Ananda Devi, Le Voile de Draupadi, Paris, L’Harmattan, 1993, p. 27.

${ }^{653}$ Ibid., p. 91.

${ }^{654}$ Ibid., p. 157.

${ }^{655}$ Ibid.

656 «Bon dié ène grand kike çose ça, mo zenfant », Ibid., p. 132.

657 « Touille-moi, touille-moi bon dié, touille-moi », Ibid., p. 51.

${ }^{658}$ Issa Asgarally, « Histoire et Linguistique : Langues, Colonialisme et Sous-Développement. Tentative de description de la superstructure linguistique de l'île Maurice de 1721 à 1982 », Journal of Mauritian Studies, 1, 1986, p. 131.

${ }^{659}$ Ibid., p. 141.

660 «Despite more than a century and a half of British rule and the imposition of English as an official language, French has maintained its position as the prestige language of Mauritius », Satish Mahadeo,
} 
parlement mauricien, l'anglais «est conservé[e] par une classe politique dont l'accession et le maintien du pouvoir sont dûs principalement à sa collaboration avec l'ancienne métropole ${ }^{661}$. Tout comme au parlement, l'anglais est la langue qui prédomine aussi à la cour de justice de l'île Maurice, car « un des décrets imposa en 1847, sous l'administration de Sir William Gomm, l'abolition de l'usage du français dans les cours de justice du pays et fit que l'anglais demeura la seule langue utilisée par les hommes de loi ${ }^{662}$. Ainsi, faisant partie de l'élite et de la classe lettrée, il n'est pas surprenant que Dev et le médecin utilisent l'anglais au sein de leur cercle. Par ailleurs, la raison pour laquelle Dev exprime son exaspération en anglais, plutôt qu'en créole ou en français, serait peut-être parce qu'il est exposé à l'anglais à longueur de journée dans son lieu de travail. Par ailleurs, Anjali note que c'est «sa langue favorite de dérobade ${ }^{663}$ suggérant que l'anglais est la langue du pouvoir, mais non des émotions. Nous constatons également que Dev et le docteur Pradhan s'expriment en anglais, non seulement parce qu'ils sont lettrés et de la classe élite, mais surtout parce que «le colonialisme anglais [qui] ne s'est jamais forcé de rendre chaque Mauricien anglophone, $[\ldots]$ a certes tenté d'augmenter le nombre de locuteurs anglophones » ${ }^{64}$ en décrétant l'anglais comme principal vecteur de l'enseignement.

Rohini Bannerjee, qui s'est intéressée à la question de langue dans Le Voile de Draupadi, observe que Devi n'utilise pas seulement le créole mais aussi le hindi et l'urdu quand ses personnages sont d'origine indienne. Elle souligne cependant que Devi n'insère pas, pour autant, beaucoup de phrases en langues indiennes. Elle ajoute que puisque l'anglais est « la langue universitaire de l'île Maurice » ${ }^{665}$ utiliser l'anglais fait que la maladie de Wynn est prise plus au sérieux. Ainsi, selon elle, Devi rend « la littérature de la région plus authentique dans son expression linguistique ${ }^{666}$.

\footnotetext{
«History of English and French languages in Mauritius: A Study in languages and power», http://www.educ.utas.edu.au/users/tle/journal/articles/Mahadeo/Mahadeo4.html, consulté le 25 février 2014.

${ }^{661}$ Issa Asgarally, « Histoire et Linguistique. Langues, colonialisme et sous-développement. Tentative de description de la superstructure linguistique de l'île Maurice de 1721 à 1982 », Journal of Mauritian Studies, 1, 1986, p. 141.

${ }^{662}$ Ibid., p. 137.

${ }^{663}$ Ananda Devi, Le Voile de Draupadi, Paris, L'Harmattan, 1993, p. 157.

${ }^{664}$ Issa Asgarally, « Histoire et Linguistique. Langues, colonialisme et sous-développement. Tentative de description de la superstructure linguistique de l'île Maurice de 1721 à 1982 », Journal of Mauritian Studies, 1, 1986, p. 142.

${ }_{665}$ Rohini Bannerjee, «L'Espace littéraire mauricien : l'hétérolinguisme dans l'œuvre francophone d'Ananda Devi », Contemporary French and Francophone Studies, 15, 2011, p. 509.

${ }^{666}$ Ibid., p. 510.
} 
Ces changements de la langue dans le roman reflètent donc bien le fait que l'anglais est la langue de travail et le français, la langue quotidienne utilisée dans les familles de classe supérieure. De plus, il est intéressant de noter que, contrairement au roman Rue la Poudrière, aucune phrase, que ce soit en créole, anglais ou bhojpuri n'est traduite dans Le Voile de Draupadi. Tant d'exemples du roman nous démontrent que la famille et tout l'entourage d'Anjali est riche mais que ce n'est tout de même pas une famille qui connaît la richesse depuis des générations. C'est en effet une famille nouvellement riche puisque le grand-père était venu d'Inde en tant que travailleur engagé, sans argent ni possession quelconque. Finalement, la question qui nous intéresse ici est de savoir si à travers ces personnages, nous voyons la présence d'un rêve mauricien. Et si oui, nous nous demandons comment et par quelles mesures ces personnages arrivent à réaliser ce rêve mauricien.

\section{Le rêve mauricien dans Le Voile de Draupadi}

Comme nous l'avons fait ressortir plus tôt, les Indo-Mauriciens de la caste supérieure se marient au sein de leur groupe, à l'instar d'Anjali et Dev, pour préserver leur culture et statut social. Mais un des buts des classes aisées est aussi de gagner beaucoup d'argent pour améliorer leur niveau de vie. Dans cette partie du travail, nous analyserons comment Dev et Faisal atteignent ce but; celui de réaliser leur "rêve mauricien".

Même si son fils est gravement malade, et que Dev «souffre, saigne à l'intérieur ${ }^{667}$, il continue de travailler beaucoup. Sa mère l'avait encouragé à travailler dur pour qu'il sorte de leur misère et Dev a pris conscience jeune qu'il fallait travailler beaucoup. Même au début de sa carrière, le jeune avocat, pas encore célèbre, «travaillait comme un forcené » ${ }^{668}$ car «au barreau, les carrières et les réputations se bâtissaient quelquefois sur un cas unique, un tour de force où l'avocat était en mesure d'exhiber son talent ${ }^{669}$. Sa réputation « si chère à ceux de sa profession » ${ }^{670}$ s'est en fait bâtie le jour où Dev a plaidé le cas de Faisal. Pour cela, Dev, se «battit de toutes ses forces, distribua des dessous-de-table et des promesses, fît disparaître un feuillet du rapport de police, trouva de faux témoins ${ }^{671}$. Ce début de réussite professionnelle constituait ainsi un premier pas vers sa réussite sociale : il « fit la une des journaux [...]

\footnotetext{
${ }^{667}$ Ananda Devi, Le Voile de Draupadi, Paris, L'Harmattan, 1993, p. 29.

${ }^{668}$ Ibid., p. 30.

${ }^{669}$ Ibid., p. 29.

${ }^{670}$ Ibid., p. 30.

${ }^{671}$ Ibid.
} 
Son intelligence développa des tentacules de ruse, son sourire prit un mordant ${ }^{672}$. Dev fait désormais partie du cercle de ceux qui détiennent le pouvoir et le succès mais en même temps il perd l'estime de sa femme qui pense que ce succès « lui aura coûté [...] la pauvre offrande de son intégrité, et l'aura définitivement dissociée de ce côté-là de sa personnalité ${ }^{673}$. Le couple se désunit et, à la fête de Faisal, Dev laisse sa femme de côté pour sceller «d'une poignée de main quelque accord avec un des invités » ${ }^{674}$ car sa réussite professionnelle compte plus que tout.

Cet engouement pour la réussite et cette soif de succès caractérisent aussi Faisal, «richissime vendeur de drogue de la capitale sous le couvert d'une petite boutique miteuse ${ }^{675}$. Pour assurer sa richesse, Faisal est prêt à tout et « de petites enveloppes bien garnies atterrissaient avec une régularité d'horloge sur les tables des fonctionnaires de police, de tous les services publics, des avocats et même certains ministres $»^{676}$.

Ainsi, Dev et Faisal ont choisi d'adopter des pratiques peu recommendables comme la corruption, pour arriver à la réussite sociale ou matérielle. Le comportement de ces deux personnages représente-il vraiment les classes aisées de la société mauricienne qui aspirent, coûte que coûte, à monter l'échelle sociale? Par l'intermédiaire de Dev et Faisal, Devi tire la sonnette d'alarme sur la corruption encore présente à l'île Maurice. En termes de corruption, d'après le Transparency International, en 2013, l'île Maurice « passe de la $43^{\mathrm{e}}$ place sur le plan mondial à la $52^{\mathrm{e}}$ avec 52 points sur 100 contre 57 l'année dernière ${ }^{677}$, ce qui démontre un certain déclin sur le plan de la corruption au sein de cette société. Ainsi, à travers ce roman, l'auteure dénonce la corruption fort réelle à Maurice ${ }^{678}$. Ces chiffres sont révélateurs et Jean-Claude Charles explique ce phénomène ainsi :

La course folle des Mauriciens moyens démontre l'empressement de pouvoir conserver son statut financier de classe moyenne et de trouver pour son compte dans cet

\footnotetext{
672 Ibid.

${ }^{673}$ Ibid., p. 31.

${ }^{674}$ Ibid., p. 53

${ }^{675}$ Ibid., p. 30.

676 Ibid.

${ }^{677}$ Afriquinfos, « Corruption : Maurice chute de neuf places à l'indice de Transparency International », http://www.afriquinfos.com/articles/2013/12/4/corruption-maurice-chute-neuf-places-lindice transparency-international-237620.asp, consulté le 27 février 2014.

678 «It is expected of fiction that it include lessons to be extracted in regard to the real world », Kai Mikkonen, «Can Fiction Become Fact? The Fiction-to-Fact Transition in Recent Theories of Fiction », Style, 40, 2006, p. 295.
} 
univers difficile $[\ldots]$ ceux qui sont à bas de l'échelle, eux se débattent comme le diable dans l'eau bénite pour se faire une place socialement acceptable, sinon ils seront la maldonne de la société ${ }^{679}$.

En fait, la relation de Dev et Faisal est celle de donnant-donnant. L'avocat aide le trafiquant de drogue à éviter l'emprisonnement, et en retour, Faisal aide Dev à être célèbre, à être accepté dans le cercle des riches et des gens influents qui détiennent un pouvoir politique. Leur cas reflète bien ce qui se passe à l'île Maurice. D'après le journal mauricien, il faut trois conditions pour faciliter la corruption : «premièrement ça prend de l'argent. Deuxièmement, il faut des personnes complaisantes qui sont redevables en raison de faveurs reçues. Troisièmement, on doit pouvoir agir à l'abri des regards ou à la faveur de silences complices ${ }^{680}$. Ceci est exactement ce que montre Le Voile de Draupadi en mettant en avant les dangers de ce "rêve mauricien" qui peut amener certains à enfreindre la loi et à bafouer leurs propres valeurs, comme Dev. De plus, d'après le Crime and Justice Security Statistics 2013, si le nombre de crimes a augmenté de 2 pour cent de 2012 à 2013, le responsable de la Police Information and Operation Room, le chef inspecteur Vinod Dawonzuth « explique que cette hausse est liée à la "fraud and dishonesty" " ${ }^{681}$. Aussi, commentant ce même fait, le sociologue Ibrahim Koodoruth avance que « de 2003 à 2007, le taux de criminalité a frôlé la barre de 45 pour cent [et] que les cas de vols, fraudes et arnaques ont connu une hausse de 5 pour cent ${ }^{682}$. Ces chiffres viennent révéler, ici, un dysfonctionnement dû à la frustration au sein de cette société, où les gens sont prêts à tout pour acquérir du bien matériel ou pour améliorer leur condition de vie.

\section{Relations de couple dans Le Voile de Draupadi}

Le Voile de Draupadi dépeint l'univers de trois générations de couples, soit le grand-père d'Anjali et sa femme, sur qui le roman n'offre pas de détail précis ; Yashoda et son mari, les parents d'Anjali et de Shyam ; Anjali et Dev ainsi que Margaret et Shyam. Chacun de ces couples issus de la même famille, présente des valeurs bien

${ }^{679}$ Jean-Claude Charles, « île Maurice, une société de décadence », http://www.defimedia.info/blog/item/2357-\%C3\%AEle-maurice-une-soci\%C3\%A9t\%C3\%A9-ded\%C3\%A9cadence.html?tmpl=component\&print=1, consulté le 27 février 2014.

680 Ibid.

${ }^{681}$ Mario Boutia, « Au cœur de l'info : la hausse dans les délits sexuels inquiète », http://www.defimedia.info/defi-quotidien/dq-actualites/item/58097-au-coeur-de-l-info-la-hausse-dansles-delits-sexuels-inquiete.html, consulté le 19 août 2014.

${ }^{682}$ Ibid. 
différentes. Nous mettrons en avant ces différences et tenterons de voir si leur situation est représentative d'une évolution dans la société mauricienne.

\section{Yashoda et son mari}

Les parents d'Anjali et de Shyam, tous deux hindous, forment un couple traditionnel. La mère, Yashoda, âgée de cinquante ans ${ }^{683}$, a toujours été « une fille de la maison, attendant d'être mariée ${ }^{684}$, ce qui s'est fait lorsqu'elle n'avait que dix-sept ans ${ }^{685}$. D'après Yashoda, son père « était l'homme le plus fanatique, le plus férocement imbu des croyances ${ }^{686}$, pour qui maintenir la tradition et sauvegarder la lignée comptait plus que tout. Il est intéressant de noter que dans le roman tout ce que l'on apprend sur le couple parental est relaté par le biais d'Anjali, qui remonte dans ses lointains souvenirs pour raconter les brefs moments de bonheur que ses parents ont connus : «père et mère tout en sourires, et retournant, eux, encore plus loin dans leur passé, et retrouvant des soirées volées à l'emprise de la belle-famille, des moments isolés de liberté où ils pouvaient se tenir la main sans contraintes ${ }^{687}$. Aussi, le roman ne donne ni le nom ni la profession de son mari. Pourtant, pour Yashoda, « il demeurait au fond d'elle des ambitions inassouvies. Ce destin d'épouse ne lui semblait pas être le sien. Tout doucement l'amertume s'est appesantie sur elle ${ }^{688}$. De son côté, le mari de Yashoda ne semble pas non plus heureux dans cette vie conjugale qui lui donne « conscience de sa propre défaite face à la vie, ou aux valeurs matérielles qui contrôlent la vie ${ }^{689}$. Le couple reste ensemble pour leurs enfants, qui les réunissent. Anjali se souvient des sorties en famille : «nos promenades, ici, père et mère assis, souriant en nous regardant, mère au sari rose d'eau flottant dans la brise ${ }^{690}$. Mais ils font chambre à part : «chacun est allé rejoindre sa chambre obscure dans la grande maison du silence ${ }^{691}$. En plus, le mari de Yashoda était un homme qui «parlait généralement si peu, et jamais de façon à révéler ses pensées profondes ${ }^{692}$, tout comme quand il était question de réagir en tant qu'homme de la maison : «mon père s'est levé, est allé s'enfermer quelque part dans la grande maison. Il refuse de participer. Il retourne

\footnotetext{
683 «A cinquante ans, il ne reste plus en elle [Yashoda] qu'un potentiel sans cesse grandissant de souffrance », Ananda Devi, Le Voile de Draupadi, Paris, L’Harmattan, 1993, p. 63.

${ }^{684}$ Ibid., p. 60.

685 « et à dix-sept ans, elle [Yashoda] a fait un mariage convenable », Ibid.

${ }^{686}$ Ibid., p. 125.

${ }^{687}$ Ibid., p. 110

688 Ibid., p. 60.

${ }^{689}$ Ibid., p. 111.

${ }^{690}$ Ibid., p. 110

${ }^{691}$ Ibid., p. 128.

692 Ibid., p. 112.
} 
fermement sa marginalité [...] mon père, d'une certaine façon, m’a tourné le dos, comme il avait tourné le dos à Shyam » ${ }^{693}$. Leur mariage avait été arrangé pour réunir deux familles plutôt que deux personnes. Mais de nature faible, le mari de Yashoda n'a pas su assumer le rôle de chef de famille qui lui était imparti et le couple vit séparément sous le même toit : «à présent, les deux occupants s'enliseront dans leur passé comme dans une machine à remonter le temps, s'y perdront, et ne retrouveront plus le chemin contigu qu'ils avaient un temps suivi et où ils pouvaient de temps à autre se tendre la main pour se reconstruire et se rassembler ${ }^{694}$. Yashoda et son conjoint ont accepté le mariage arrangé pour se plier aux normes de leur ethnie mais leur union est un échec sur le plan personnel.

\section{Anjali et Dev}

Anjali a rencontré Dev «au mariage d'une cousine ${ }^{695}$ lorsqu'elle n'avait que dix-huit ans. Quand les deux familles se rencontrent pour officialiser la relation, Anjali, pensant que Dev est le compagnon parfait pour elle ${ }^{696}$, accepte cette union, prenant «pour le destin ce qui n'était en fait qu'une absence de choix ${ }^{697}$. Contrairement à Anjali, Dev ne ressent pas d'attirance et lors de cette rencontre familiale, au lieu de participer à cet événement, il « regardait avec ennui par la fenêtre, perdu dans ses rêves de puissance ${ }^{698}$. Anjali reconnaît que le couple s'est, en fait, laissé guider « de manière occulte, par la famille ${ }^{699}$. Malgré tout le couple s'aime et connaît des moments de tendresse au début de leur mariage : «il m'entoura de son bras [...] il me serra contre lui et m'embrassa ${ }^{700}$. Mais Dev et Anjali s'éloignent l'un de l'autre au moment de la jaunisse néonatale de leur fils puis, au moment de sa méningite aiguë. Un autre problème qui déchire ce couple survient lorsque Dev s'associe à Faisal, un puissant trafiquant de drogue que la jeune femme n'apprécie pas. Anjali finit par s'opposer à son mari et à toute sa belle-famille quand ils lui demandent de faire le sacrifice de la marche sur le feu pour sauver l'enfant. Anjali s'aperçoit alors que « Dev, lui aussi, est à présent du mauvais côté ${ }^{701}$ et réalise à quel point leur vie est régie par les autres membres de sa famille, par des traditions, des normes sociales, des règles et

\footnotetext{
${ }^{693}$ Ibid., p. 124.

${ }^{694}$ Ibid., p. 128.

${ }^{695}$ Ibid., p. 20.

${ }^{696}$ «j'avais cru l'aimer, depuis notre première rencontre», Ibid.

${ }^{697}$ Ibid.

${ }^{698}$ Ibid

${ }^{699}$ Ibid

${ }^{700}$ Ibid., p. 21.

${ }^{701}$ Ibid., p. 129.
} 
des conventions qui imposent à la femme d'être soumise à la famille : «toute ma vie, j'ai été à l'écoute des autres [...] cela aussi, la tradition nous l'enseigne dès l'enfance ; une femme doit penser avant tout à son mari, à ses enfants, à ses parents. Mais à ellemême, jamais ${ }^{702}$. Ne pouvant plus tolérer ce fardeau, Anjali décide de se libérer des chaînes familiales qui l'emprisonnent : «à partir de maintenant, enfin, finalement, ma vie m'appartient. Je n'ai de comptes à rendre à personne ${ }^{703}$. Après la mort de son fils, elle demande à son mari « de partir pour de bon ${ }^{704}$, même si ce dernier «a pleuré, supplié ${ }^{705}$ pour rester aux côtés de sa femme.

Ces deux personnages, dont la personnalité est différente, n'ont pu trouver un juste milieu pour faire fonctionner leur couple. Si Anjali, jeune fille, rêvait d'amour, elle n'a eu «aucun choix, aucune autre voie à suivre que celle de sa condition de femme ${ }^{706}$. Dev, pour sa part, a toujours eu la tête sur les épaules, et croyait que le principal était d'acquérir un statut social élevé et d'amasser de l'argent. Ces valeurs discordantes entre mari et femme nous rappellent les théories de "function" et "dysfunction" de Merton. Ainsi, ce qui était fonctionnel pour Anjali était dysfonctionnel pour Dev, et ce qui était fonctionnel pour Dev était dysfonctionnel pour Anjali. Voulant se libérer des règles imposées par la société et la famille, Anjali se rebelle parce que, comme l'explique Merton, ce conflit de valeurs la pousse à prendre une décision qui va à l'encontre des conventions ${ }^{707}$ du mariage arrangé.

Les mariages arrangés, comme celui d'Anjali et de Dev, sont en effet une réalité de l'île Maurice où une grande majorité des parents, et surtout ceux qui sont les descendants d'Indiens, décident du mariage de leurs enfants pour assurer la continuité de leurs pratiques culturelles et ainsi préserver leur identité ethnique ${ }^{708}$; pratique confirmée par Ari Nave ${ }^{709}$. Comme Anjali et Dev qui ont essayé de trouver le bonheur

\footnotetext{
702 Ibid., p. 154.

703 Ibid., p. 165.

${ }^{704}$ Ibid., p. 174.

705 Ibid., p. 173.

${ }^{706}$ Ibid., p. 61.

707 «It is the conflict between culturally accepted values and the socially structured difficulties in living up to these values which exerts pressure toward deviant behaviour and disruption of the normative system », Robert Merton, Social Theory \& Social Structure, New York, The Free Press, 1968, p. 245.

${ }_{708}$ «But people in Mauritius clearly do not select spouses at random, choosing instead to marry endogamously, passing their culture to their children along with their ethnic identity », Ari Nave, «Marriage and the Maintenance of Ethnic Group Boundaries: the Case of Mauritius », Ethnic and Racial Studies, 23, 2000, p. 331-332.

709 «Arranged marriages and pseudo-arranged marriages remain strong among people of Indian descent in Mauritius », Ibid;, p. 342.
} 
tout en acceptant la volonté de leurs parents, de nombreux jeunes Mauriciens cèdent à la pression de leurs parents et acceptent leur choix de conjoints ${ }^{710}$.

Les parents d'Anjali et Dev, en unissant deux jeunes issus des mêmes antécédents familiaux, leur assurent une vie sans complication et rejettent surtout toute possibilité de divorce car ces deux personnes sont censés être compatibles et se trouver sur la même longueur d'onde. C'est aussi le phénomène qu'observe Ari Nave dans la société mauricienne ${ }^{711}$. Mais cette pratique culturelle conduit assez souvent à la séparation, comme pour Anjali et Dev. De fait, «la justice mauricienne prononce en moyenne cinq jugements de divorce par jour $»^{712}$ et le nombre de divorces continue de grimper en flèche «passant de 1729 en 2011 à 1930 en $2012 »^{713}$. Dans Le Voile de Draupadi, c'est Anjali qui demande la séparation après sept ans de mariage ${ }^{714}$. D'après Loga Virahsawmy, la directrice de Gender Links, dans la réalité «ce sont parfois les femmes qui décident de prendre l'initiative pour mettre fin à leur mariage $»^{715}$ et $« 50$ pour cent des cas concernent des couples ayant moins de dix ans de mariage ${ }^{716}$. C'est aussi ce que semble affirmer un journal quotidien mauricien :

Le nombre de divorces ne cesse d'augmenter à Maurice. En 2013, selon le dernier rapport annuel du judiciaire, 2450 demandes de divorces ont été déposées à la Family Division de la Cour suprême, soit une hausse de 2 pour cent et les demandes se font plus par les femmes que les hommes, soit 48 pour cent contre 28 pour cent respectivement ${ }^{717}$.

\footnotetext{
710 «In Mauritius, wishes of the parents carry great weight on the spouse choices of their children », Ibid., p. $12-13$.

711 « Mauritian parents have an invested interest in the successful marriage of their children. They often fear that marriage to a member of another ethnic group will result in divorce. Whether true or not, a general conception seems to exist in Mauritius and elsewhere that cultural incompatibilities are the leading cause of marital conflict and divorce among inter-ethnic couples », Ibid., p. 341.

712 Linfo.Re, Antenne Réunion Télévision, «Hausse du nombre de divorces à Maurice », http://www.linfo.re/049-Ocean-Indien/402-Societe/496065-Hausse-du-nombre-de-divorces-a-Maurice, consulté le 3 mars 2014.

713 Ibid.

714 « Aujourd'hui, sept ans plus tard, je regarde mon mari à mes côtés », Ananda Devi, Le Voile de Draupadi, Paris, L'Harmattan, 1993, p. 23.

${ }^{715}$ Linfo.Re, Antenne Réunion Télévision, « Hausse du nombre de divorces à Maurice », http://www.linfo.re/049-Ocean-Indien/402-Societe/496065-Hausse-du-nombre-de-divorces-a-Maurice, consulté le 3 mars 2014.

716 Ibid.

${ }^{717}$ Nilen Kattany, «Bilan du judiciaire pour 2013 : Les femmes demandent le divorce plus que les hommes », http://www.defimedia.info/live-news/item/53682-bilan-du-judiciaire-pour-2013-les-femmesdemandent-le-divorce-plus-que-les-hommes.html, consulté le 31 mai 2013.
} 
Outre l'incompatibilité et le manque de communication dans le couple, ce sont aussi les valeurs de la société patriarcale qui poussent Anjali à quitter son mari qui n'a pas hésité à la violer ${ }^{718}$. Cette question de viol conjugal est une réalité qui perdure à l'île Maurice et dans de nombreuses sociétés patriarcales, malgré les lois qui l'interdisent. Ainsi, d'après les Human Rights Reports, la loi mauricienne interdit le viol mais ne reconnait pas le viol conjugal ${ }^{719}$.

Le couple Anjali et Dev, dominé par les circonstances et les conventions sociales hindoues patriarcales finit par se séparer à cause de leur incompatibilité. Leur mariage est arrangé, à l'image de ce qui ce passe actuellement dans la société mauricienne, où les mariages arrangés sont contractés pour assurer la réussite financière et sociale du couple, est un échec.

\section{Margaret et Shyam}

Shyam a rencontré Margaret à la fin de ses études secondaires, alors qu'elle avait «à peine vingt ans ${ }^{720}$. Tous deux ont très vite réalisé qu'ils avaient des affinités. Malheureusement, ce couple a souffert de la désapprobation des autres, surtout des membres de la famille de Shyam parce que Margaret, une fille créole, n'est ni hindoue, ni de caste supérieure. En particulier, si les parents de Shyam s'opposent à cette relation, c'est aussi parce qu'ils pensent qu'en se mariant à une Créole, leur fils mettrait fin à cette tradition d'origine indienne de se marier au sein du même groupe social, et interromprait la lignée. Aussi, Shyam et Margaret se rencontrent-ils «à l'abri des regards $»^{721}$. Finalement, Shyam a loué « un appartement en ville, où il serait libre de vivre avec son amie sans même l'épouser ${ }^{722}$ puisque Margaret avait déjà été mariée à l'âge de seize ans et que son mari, violent ${ }^{723}$, l'a quittée sans divorcer ${ }^{724}$. Shyam et Margaret sont unis par leur expérience du malheur : Margaret a connu « une existence sans protection, sans affection, avec tous les coups et les chocs de la misère et de

\footnotetext{
718 « J'avais cette certitude, cette effroyable accusation au fond de moi, que je venais d'être violée », Ananda Devi, Le Voile de Draupadi, Paris, L’Harmattan, 1993, p. 57.

719 «The law prohibits rape, but there is no provision for spousal rape », Bureau of Democracy, Human Rights and Labour, « 2013 Human Reports Rights: Mauritius », http://www.state.gov/j/drl/rls/hrrpt/2013/af/220139.htm, consulté le 24 mars 2014, p. 11.

${ }^{720}$ Ananda Devi, Le Voile de Draupadi, Paris, L'Harmattan, 1993, p. 144.

${ }^{721}$ Ibid., p. 19.

722 Ibid., p. 120.

723 « Je sais qu'elle a été battue par son premier mari », Ibid., p. 143.

724 « Nous avions fini par apprendre que Margaret avait été mariée à l'âge de seize ans et que son mari l'avait quittée sans lui donner le divorce », Ibid., p. 122.
} 
l'existence sordide ${ }^{725}$ tandis que Shyam a vécu avec le fardeau de la mort de Vasanti sur la conscience.

Si les parents de Shyam pensent que le couple est nécessairement voué à l'échec, il vit dans l'harmonie malgré les difficultés. Contrairement au mariage arrangé d'Anjali et de Dev, Shyam et Margaret sont unis par l'amour et se sont bâti un monde bien à eux. Shyam explique à Anjali qu'ils sont «perdus dans ce monde-là. C'est pour ça [qu'ils sont] bien ensemble. Chacun est le repère de l'autre ${ }^{726}$. Quand le mari de Margaret lui accorde le divorce, Shyam et Margaret, sûrs de leur amour l'un pour l'autre, décident de se marier ${ }^{727}$. Si le mariage d'Anjali et de Dev a débuté dans le bonheur et l'accord des familles, il a fini par une séparation, tandis que celui de Shyam et de Margaret a connu des débuts très difficiles. Le couple a su surmonter tous les hauts et bas bien que Shyam se soit opposé à ses parents et ait rejeté les valeurs sociales de sa caste qui n'autorise pas les mariages mixtes.

Le cas de Shyam et Margaret qui vont à l'encontre des règles sociales pour vivre selon leurs propres valeurs est-il une exception à l'île Maurice ? D'après Ari Nave, les mariages mixtes sont rares à cause de la pression sociale qui dicte qu'il faut se marier au sein de son groupe ethnique ${ }^{728}$ et social. En fait, à l'île Maurice, quatre couples sur cinq se marient au sein de leur groupe social, comme la tradition le veut. Tout comme leurs aînés, les jeunes Mauriciens, d'après Nave, prennent toujours exemple dans leur cercle immédiat ${ }^{729}$ parce qu'ils ont peur de représailles ou de punitions de la part de ceux qui leur sont chers ${ }^{730}$. Bien sûr, comme Shyam et Margaret, il y a des exceptions dans la société mauricienne, surtout parmi les Indo-Mauriciens ${ }^{731}$.

\footnotetext{
${ }^{725}$ Ibid., p. 22.

726 Ibid., p. 145.

727 « Tu sais le type, il va partir [...] il va donner le divorce à Margaret [...] aussitôt que ce sera fait, nous allons nous marier », Ibid., p. 163.

${ }_{728}$ «Inter-marriage rates are low because people have evolved a predisposition for conservative decisionmaking », Ari Nave, "The Role of Marriages in the Maintenance of Ethnic Group Boundaries : The Case of Mauritius », http://arinave.com/arinavec/wp-content/uploads/2012/05/pan-african1.pdf, consulté le 28 février 2014, p. 8.

${ }^{729}$ «Marriage in Mauritius as most elsewhere, is a decision which young unmarried individuals must make on the basis of little personal experience [...] it should come to no surprise, then, that people relay heavily on culturally learned preferences and beliefs in shaping their decision on whom to marry. In other words, a heuristic mechanisms has evolved where people incorporate the most frequent occurring cultural beliefs found in the immediate environment », Ibid., p. 9.

${ }^{730}$ «There does not seem to be strong indication that the rate of inter-ethnic marriage is rapidly increasing [...] This is because individuals act within a context of constraints, particularly the threat of punishment from immediate family members for entering into an inter-ethnic marriage », Ari Nave, " Marriage and the maintenance of ethnic group boundaries: the case of Mauritius », Ethnic and Racial Studies, 23, 2000, p. 339.

731 «One would expect to find a constant pattern of predominately ethnic endogamy but where intermarriage always occurred. And this is what we find in Mauritius », Ari Nave, « The Role of Marriages in
} 
Mais, le cas de Shyam et Margaret ne représente qu'une minorité ${ }^{732}$ alors que le mariage arrangé d'Anjali et de Dev représente la majorité. Dans le roman, cette notion traditionnelle de mariage arrangé, comme celui de Yashoda, est remise en question puisque le couple Anjali-Dev divorce et que le couple mixte Shyam-Margaret reste uni en dépit de la désapprobation familiale et sociale. De plus, Anjali aussi bien que Margaret, sont celles qui ont décidé de prendre leur vie en main, malgré les difficultés à venir, contrairement à Yashoda qui accepte son sort. Cette évolution montre une ouverture de la jeune génération qui commence à rejeter les valeurs de leurs parents et grand-parents.

\section{Représentation réaliste à la fois locale et universelle des ethnies et classes sociales dans Le Voile de Draupadi}

Tout compte fait, Le Voile de Draupadi souligne une disparité entre Hindous et Créoles qui trouve sa source dans les croyances et les coutumes venues d'Inde. Ces traditions qui renforcent la ségrégation des Créoles mais qui permettent de préserver la lignée et la réussite sociale des Indo-Mauriciens sont remises en cause par la dernière génération qui privilégie plus le bonheur personnel que les traditions. Ainsi, les mariages arrangés sont perçus comme échec contrairement au couple mixte qui se marie par amour. Cette évolution de mentalité dans la communauté indo-mauricienne est reflétée dans les mariages inter-ethniques ou les unions libres qui ont pris de l'ampleur dans la société mauricienne. Le roman retrace avec réalisme ces changements sociaux récents à travers les trois générations de la famille d'Anjali.

\section{Le Sari vert}

Pour Le Sari vert, tout comme dans Le Voile de Draupadi, l'étude sera axée principalement sur la représentation des communautés hindoue et créole et sur le registre de langue utilisé par les personnages. Nous nous intéresserons aussi aux relations du couple de Bissam, de Kitty et son mari, et de Malika et Marie-Rose. Mais avant, nous récapitulerons ce que les chercheurs ont dit à ce propos.

the Maintenance of Ethnic Group Boundaries : The Case of Mauritius », http://arinave.com/arinavec/wpcontent/uploads/2012/05/pan-african1.pdf, consulté le 28 février 2014, p. 9-10.

732 «Clearly, Mauritians have a preference for marrying someone of the same ethnic group », Ari Nave, « Marriage and the Maintenance of Ethnic Group Boundaries: the Case of Mauritius », Ethnic and Racial Studies, 23, 2000, p. 334. 
S'il y a beaucoup d'analyses sur Rue la Poudrière et le Voile de Draupadi, à notre connaissance, il n'existe que quelques comptes-rendus critiques sur Le Sari vert.

Magali Marson pense que le roman montre «l'envers sombre [...] des cartes postales ${ }^{733}$ où « l'île Maurice est mise en abyme ${ }^{734}$. Pour Marine de Tilly, l'auteure dépeint un « vieux médecin ivrogne et bavard, raciste, violent, misogyne et allergique à toute forme de modernité, surtout quand il s'agit du statut des femmes qu'il a toujours traitées en moins que rien ${ }^{735}$ et présente « une réflexion bouleversante sur les rapports de pouvoir et de domination $»^{736}$. Le quotidien mauricien, Le Matinal, juge aussi que «plus que la condition féminine, c'est la condition humaine qu'Ananda Devi juxtapose dans le drame de ces trois êtres ${ }^{737}$. Pour ce critique, Ananda Devi « nous dit que notre île "moderne" est en train d'engendrer des monstres. Des êtres comme vous et moi, mais qui dans le secret de leur intimité violent l'innocence de nos enfants ${ }^{738}$. Christine Rousseau, pour qui Le Sari vert est «direct, dru ${ }^{739}$, parle du long «monologue, éminemment violent, terrible et surtout dérangeant ${ }^{740}$ d'un médecin qui fait souffrir et domine les femmes et qui est finalement « à la merci de deux femmes : Kitty, sa fille, élevée dans le désamour, la peur et la soumission à cet ogre paternel, et Malika, sa petite-fille rebelle et provocante dans son amour des femmes ${ }^{741}$. FlorinaLiliana Mihalovici remarque que Devi «dénonce le poids des traditions dans une société multiculturelle et multiraciale qui pèse sur le genre féminin dans tous ses états $\gg^{742}$.

Se concentrant sur le martyre des femmes et surtout celui du médecin, Karine Bénac-Giroux écrit que le médecin «souhaite une épouse soumise alors qu'il est amoureux d'une femme-enfant $»^{743}$. Elle compare la mère du médecin à Cendrillon

\footnotetext{
${ }^{733}$ Magali Marson, «Ananda Devi - Le Sari vert», Cultures Sud. La revue en ligne des littératures du sud, http://www.culturessud.com/contenu.php?id=42, consulté le 2 mai 2012.

734 Ibid.

${ }^{735}$ Marine de Tilly, «Le Sari vert, d'Ananda Devi », http://www.lepoint.fr/livre/le-sari-vert-d-anandadevi-08-02-2011-1293168_79.php, consulté le 19 août 2013.

736 Ibid

${ }^{737}$ Le Matinal News Service, «Le Sari vert d'Ananda Devi : une constance littéraire qui force l'admiration », Le Matinal, 2009, http://www.lematinal.com/mobile/magazine/268-sari-vert-dAnandaDevi-une-constance-littraire-qui-force-ladmiration.html, consulté le 2 mai 2012.

738 Ibid.

${ }^{739}$ Christine Rousseau, «Le Sari vert d'Ananda Devi : un terrible monologue », http://www.lemonde.fr/livres/article/2009/11/12/le-sari-vert-d-ananda-devi_1266068_3260.html, consulté le 12 avril 2013.

${ }^{740}$ Ibid.

${ }^{741}$ Ibid.

${ }^{742}$ Florina-Liliana Mihalovici, « La "Guerre silencieuse" de la femme dans une société patriarcale qui la dévore : Le Sari vert d'Ananda Devi », dans Parole au silence, Limoges, Pulim, 2012, p. 103.

${ }^{743}$ Karine Bénac-Giroux, «Le martyre des femmes dans Le Sari vert d'Ananda Devi », dans Libertés et Oppressions, Paris, L’Harmattan, p. 105.
} 
«dont le fils seul pourrait un jour, en bon prince charmant, la délivrer de la pauvreté ${ }^{744}$. Elle affirme que la femme de Bissam « en refusant jusqu'au bout de cuire le riz convenablement, $[\ldots]$ développe une stratégie de résistance étrangement efficace, tellement d'ailleurs qu'elle en mourra, à force de pousser à bout son époux ${ }^{745}$. Pour elle, la société mauricienne doit faire face au «vieux thème de la femme battue ${ }^{746}$ « pour accéder à une véritable émancipation en passant par la reconnaissance d'un état de fait ancien, à abolir $»^{747}$.

Pour Rohini Bannerjee, le vieil homme de quatre-vingts ans «ne cache jamais sa haine pour les trois femmes qui l'entourent [et] ironiquement, [...] a besoin d'elles pour éviter la solitude de son agonie ${ }^{748}$. Mais les trois femmes « réussissent à survivre à l'abus et la torture ; elles résistent à travers le silence ${ }^{749}$. Pour elle, Devi démontre «la beauté de l'humanité et la puissance de la femme » ${ }^{750}$ en écrivant « une tragédie universelle qui pourrait être connue et vécue bien au-delà de l'île Maurice $»^{751}$. Tout comme Bannerjee, Mihalovici est d'avis que dans ce roman, « le silence devient [...] la seule arme viable contre une avalanche inattendue de coups de pieds, de poings, de mots blessants et méprisants ${ }^{752}$. Ainsi, si pour Kitty ce silence «n'est plus une forme de protection, mais la plus naturelle réponse à la violence du père ${ }^{753}$, Malika, elle, « comprend que la confrontation ne peut pas être remportée par le silence » ${ }^{754}$ et c'est pour cela qu'elle « prend le rôle de mâle, de protecteur contre l'autre homme, s'attirant encore plus le mépris du grand-père qui malgré la violence de son langage et des faits racontés, dévoile une certaine peur et méfiance à l'égard de Malika ${ }^{755}$. Mihalovici remarque qu'en «échappant aux poids des traditions et des préjugés, la femme peut initier sa rébellion contre tout ce qui peut la soumettre par le droit à l'expression, par la parole ${ }^{756}$.

\footnotetext{
${ }^{744}$ Ibid., p. 109.

745 Ibid., p. 111.

746 Ibid., p. 113.

${ }^{747}$ Ibid.

${ }^{748}$ Rohini Bannerjee, «Le Sari vert », Journal du conseil international d'études francophones (CIEF), 25, 1, 2010.

${ }^{749}$ Ibid.

750 Ibid.

751 Ibid.

${ }^{752}$ Florina-Liliana Mihalovici, «La "Guerre silencieuse" de la femme dans une société patriarcale qui la dévore : Le Sari vert d'Ananda Devi », dans Parole au silence, Limoges, Pulim, 2012, p. 105.

753 Ibid., p. 107.

${ }^{754}$ Ibid., p. 109

755 Ibid., p. 110

${ }^{756}$ Ibid., p. 112.
} 
Yves Chemla, quant à lui, suggère que l'auteure est « allée chercher la matière dans les replis les plus obscurs de sa conscience ${ }^{757}$ et que Le Sari vert 《luit de cette beauté qu'ont les visages réduits au silence par la folie masculine, mais dont les espaces intérieurs sont balayés d'émotions que les mots même insuffisants, parviennent quand même à repeupler » ${ }^{758}$. Pour lui, ce roman est « comme un combat réussi contre la misère, mené par le jeune homme, appuyé par sa mère, qui ne verra pas la conquête ${ }^{759}$. Il termine en ajoutant qu' « Ananda Devi répond à une interrogation radicale sur la condition féminine, et le façonnement de celle-ci par les pseudo-morales et les échardes de l'histoire qui façonnent l'île Maurice ${ }^{760}$.

Nous constatons donc que la plupart de ces critiques mettent en avant les caractéristiques misogynes du vieil homme et le silence des personnages femmes soumises, éléments qui seraient symptômatiques de la société patriarcale mauricienne, sans pour cela traiter des différences ethniques et sociales qui existent dans les diverses communautés. Nous viserons à combler cette lacune en examinant la représentation des ethnies, de la classe sociale et des relations de couple.

\section{La représentation des différentes ethnies dans Le Sari vert}

Le Sari vert met en scène la famille du docteur Bissam, une famille hindoue, et dresse le portrait de Marie Rose Patience, la jeune femme créole qui est devenue la compagne de Malika, la petite-fille du médecin. Le Voile de Draupadi et Le Sari vert présentent donc des thèmes similaires, et des problèmes identiques. Tout comme dans Le Voile de Draupadi, Le Sari vert fait le tour d'une famille hindoue et contient ainsi des éléments qui rapprochent ce roman à la coolitude. Nous étudierons donc comment sont perçues les communautés hindoue et créole du point de vue social à travers les personnages.

\footnotetext{
${ }^{757}$ Yves Chemla, « Ananda Devi: Le Sari vert», http://www.cec-ong.org/index.php?option=com_content\&view=article\&id=416:ananda-devi-le-sari-vert205-\&catid=131:chroniques-de-livres\&Itemid=224, consulté le 11 avril 2013.

$\frac{205-\& \text { ciid }}{758}$ Ibid

${ }^{759}$ Ibid

${ }^{760}$ Ibid.
} 


\section{La communauté hindoue dans Le Sari vert}

Dans un de ses soliloques, le vieil homme déclare que sa famille est « de bonne souche indienne ${ }^{761}$. Même les noms des personnages, comme Bissam ou Kaveri Bhavani « (qui est le «nom de reine de la lignée solaire Kuru) ${ }^{762}$ indiquent que ces personnages sont hindous. Le Sari vert, tout comme Le Voile de Draupadi, dépeint les personnages hindous comme étant beaux, bien habillés et cultivés. Ainsi, la femme du médecin adorait les saris de couleur pastel : « un jour elle était bleu ciel, un autre jour rose bonbon, un autre jour encore vert eau ${ }^{763}$. Elle portait aussi «ses boucles d'oreilles longues, son diamant au nez ${ }^{764}$. La jeune femme était tellement belle qu'elle est comparée à Soraya, une actrice indienne, et certains prétendaient même qu'elle «était plus belle que Soraya [qu'elle] était une flamme ambulante sur ses sandales à talons hauts ${ }^{765}$. Outre la mère, Kitty, la fille du couple, est aussi décrite par les autres comme étant belle avec les «yeux clairs ${ }^{766}$. Nous retiendrons donc les mêmes caractéristiques de la caste supérieure hindoue que dans Le Voile de Draupadi qui sont révélatrices de la réalité mauricienne.

Comme souligné plus tôt, l'idée de la coolitude ne se limite pas au départ des travailleurs engagés de l'Inde mais concerne aussi et surtout le maintien, dans leur nouvelle société, de leurs rites et traditions et de leur désir de se faire une meilleure place dans la société par le travail. Le Sari vert fait certainement allusion à cette notion par le biais de Bissam. Entouré de misère, surtout après la mort de sa mère, Bissam tente avec acharnement de sortir de sa condition et de se créer une identité à lui. Il a été tellement diligent, que ses patients l'ont même nommé le Docteur Dieu. D'après Happymon Jacob, cette attitude attachée à la coolitude relève de la perte d'identité ou de statut social ${ }^{767}$. Cette idée trouve sa source dans l'étude de Khal Torabully qui avance que même pour les travailleurs engagés, un des buts était d'acquérir des lopins de terre afin de s'y installer. Ainsi faisant, ils allaient s'assurer sécurité et stabilité

\footnotetext{
761 Ananda Devi, Le Sari vert, Paris, Gallimard, 2009, p. 13.

762 Ibid., p. 57.

${ }^{763}$ Ibid., p. $57-58$.

${ }^{764}$ Ibid., p. 25.

765 Ibid., p. 26- 27.

766 Ibid., p. 13

767 «It is a story of losing one's history and identity, looking desperately for them and then at last recreating them with the resources available », Happymon Jacob, « Home and Displacement: Refugees, Diaspora and India », http://sas.sagepub.com/content/11/1/100.full.pdf+html, consulté le 10 mars 2014, p. 103.
} 
politique et sociale ${ }^{768}$. Pour Bissam, acquérir une maison et se faire reconnaître en tant que médecin lui assurait un bon statut social et un bon revenu. Même si Bissam est devenu un médecin de renom, cette notoriété n'a pas suffi à convaincre les parents de sa bien-aimée pour qu'ils acceptent leur union. Pour cette approbation, il a fallu « de longs mois de négociations ${ }^{769}$. Donc tout comme Bissam qui, étant médecin, a dû quand même négocier avec les parents de sa bien-aimée, les coolies, d'après Torabully, étaient sous-estimés par les autorités, même s'ils avaient un contrat à leur nom, qui authentifiait leur identité. Et c'est ce qui les forçaient à lutter pour obtenir leurs droits légaux et leurs statut social ${ }^{770}$. Pour Vicram Ramharai, même après des décennies, les descendants des travailleurs engagés indiens continuent à rechercher le respect et la reconnaissance ${ }^{771}$, exactement comme le docteur Bissam dans le roman.

Finalement, ce roman démontre un docteur qui a acquis du bien matériel et du respect. De ce fait, cette lutte pour l'appartenance du médecin est identique à celle des coolies, qui ont pu se faire une place dans la société ${ }^{772}$. C'est ainsi que Bissam est désigné comme «l'exemple du vrai Mauricien » ${ }^{773}$, signe de reconnaissance et de respect.

\section{La communauté créole dans Le Sari vert}

Le Sari vert, tout comme Le Voile de Draupadi, présente les disparités sociales entre les communautés hindoue et créole. Dans Le Sari vert, le vieux médecin montre sa haine envers la compagne de Malika qui est créole, alors que Malika fait ressortir ses qualités.

Pour Malika, Marie-Rose « est bien belle, et elle est bien patiente, ses yeux sont lourds de toute la patience dont elle a eu besoin depuis sa naissance pour être et grandir

\footnotetext{
768 «One pragmatic reaction of the indentured labourer was to aspire to possess the land, and this was in keeping with rural Indian culture, even if, in exile, there was greater social and economic mobility in the process. This ownership was seen by the indentured as the means of procuring effective, political and cultural security », Marina Carter, Khal Torabully, Coolitude. An Anthology of the Indian Labour Diaspora, London, Anthem Press, 2002, p. 162.

${ }^{769}$ Ananda Devi, Le Sari vert, Paris, Gallimard, 2009, p. 24.

770 "The coolie himself was treated as "object". And even though he had his name on a contract, he had to fight to make his humanity respected. But the very fact of having this name on the contract is a highly significant aspect of coolitude, bequeathing on him a human status », Marina Carter, Khal Torabully, Coolitude. An Anthology of the Indian Labour Diaspora, London, Anthem Press, 2002, p. 160.

${ }^{771}$ Vicram Ramharai, « Récits postcoloniaux, retour colonial et diaspora indienne à Maurice », http://revel.unice.fr/symposia/actel/?id=417, consulté le 11 juillet 2014.

${ }_{772}$ «But, ultimatelly, the coolie became involved in the building of a new identity in the land where he/she settled », Marina Carter, Khal Torabully, Coolitude. An Anthology of the Indian Labour Diaspora, London, Anthem Press, 2002, p. 191.

${ }^{773}$ Ananda Devi, Le Sari vert, Paris, Gallimard, 2009, p. 131.
} 
et devenir celle qu'elle est ${ }^{774}$. Malika admire surtout le courage de Marie-Rose grâce auquel elle a pu surmonter sa misère : «oui, mon admiration est comme au premier jour, lorsqu'elle est venue travailler dans cette école, enveloppée d'un châle qui cachait sa misère, qui cachait son corps, qui cachait sa générosité et sa tristesse ${ }^{775}$. Malika aime les «formes molles ${ }^{776}$ de Marie-Rose et aussi le fait qu'elle soit une femme «bien noire $[\ldots]$ une magnifique Noire $»^{777}$.

Par contre aux yeux du docteur Bissam, Marie-Rose est une «vache noire » ${ }^{778}$ qui a une «lourde démarche de vache ${ }^{779}$. Il imagine que «peut-être [ses] déjections sont plus parfumées qu'elle ${ }^{780}$ : il trouve qu'elle est grosse ${ }^{781}$ et qu'elle lui adresse la parole «dans la langue des pauvres ${ }^{782}$. Cette haine du vieux envers Marie-Rose provient plus du fait que Marie-Rose est créole que du fait qu'elle est la compagne de sa petite-fille. Pour lui, être créole équivaut à être vilain, gros, puant, paresseux et pauvre. A ses yeux, Marie-Rose représente tous les Créoles dont il a une opinion très négative : «il n'y a qu'eux pour marcher comme ça, avec cette cadence-là, je me comprends, un rythme qui n'est pas un rythme, qui est une sorte de paresse d'être si profonde qu'il fallait l'esclavage pour les faire travailler ${ }^{783}$.

\section{Les disparités raciales et discriminatoires dans Le Sari vert}

Un tel dénigrement des Créoles est-il vraisemblable? L'attitude de Malika dénote-t-elle un changement vis-à-vis des Créoles dans la société mauricienne ? En fait, nous l'avons dit, les idées reçues du vieil homme remontent aux anciens préjugés sur les esclaves noirs. Bernardin de St Pierre ${ }^{784}$ avait remarqué ces stéréotypes discriminatoires à l'île Maurice au XVIII ${ }^{\mathrm{e}}$ siècle. Depuis, ce racisme et cette

\footnotetext{
${ }^{774}$ Ibid., p. 46.

775 Ibid.

776 Ibid.

777 Ibid., p. 47.

778 Ibid., p. 49

779 Ibid., p. 52.

${ }^{780}$ Ibid., p. 49.

781 «...] sa grosse femme (grosse ? je ne sais si elle a dit qu'elle était grosse, mais c'est ainsi que je la vois) », Ibid., p. 50.

${ }^{782}$ Ibid., p. 52.

783 Ibid.

784 «In Mauritius, the traveller accounts of Bernardin de St Pierre (in the 1700s in Mauritius), while producing a very rich account of Mauritius society in those early years, reinforced literary stereotypes of blacks as labourers and slaves and whites as owners and managers », Truth \& Justice Commission, «Report of the Truth and Justice Commission. Volume 3. Contemporary History, Culture and Society.Research Reports, Technical Studies and Surveys », http://pmo.gov.mu/English/Documents/TJC_Vol3.pdf, consulté le 13 mars 2014, p. 532.
} 
discrimination envers les Créoles sont devenus monnaie courante à l'île Maurice ${ }^{785}$, d'autant plus qu'il profite au système capitaliste qui compte sur une classe inférieure et opprimée pour faire le dur labeur ${ }^{786}$.

Comme le suggère Le Voile de Draupadi, ceux qui sont issus de la caste supérieure sont censés être des gens riches au teint clair. Or, nous constatons que la mère du médecin était une femme très pauvre et le médecin a dû travailler avec acharnement pour grimper à l'échelle social. Et c'est grâce à son mariage, qu'il a pu accéder à la caste supérieure. Cette contradiction pourrait relever du fait que, comme le démontre la Truth and Justice Commission, lors du voyage des coolies de l'Inde à Maurice, il s'était formé des couples issus de différentes castes, dans le but d'augmenter en nombre et d'accéder à la caste supérieure pour monter l'échelle sociale et échapper à la vie misérable de la caste inférieure ${ }^{787}$. L'attitude du vieil homme est conforme aux normes de la société mauricienne, dans laquelle, les Mauriciens ont très souvent recours à un discours superficiel et classifient les gens selon des critères $\operatorname{raciaux}^{788}$. Comme le vieil homme, beaucoup de Mauriciens ont recours à 1'“Aversive Racism", en utilisant de façon délibérée, un langage hostile et des propos raciaux ou en ayant des comportements indignes, envers une personne de couleur pour porter atteinte à sa dignité ${ }^{789}$. Ce phénomène est confirmé par Rosabelle Boswell dans le rapport de la Truth and Justice Commission qui précise que les Créoles, descendants d'esclaves, sont souvent victimes d'attaques raciales à l'île Maurice ${ }^{790}$, même si de nombreux Mauriciens voudraient éradiquer cette discrimination raciale et sensibiliser les habitants sur les résultats dévastateurs que cette mentalité peut causer chez la personne ciblée ${ }^{791}$.

\footnotetext{
785 «Utterance coming from respectable citizens or a closer look at school text-books or newspaper articles can reveal the persistence of racism in Mauritius », Ibid., p. 531.

${ }^{786}$ «It reaffirms discourse of superiority and inferiority and it maintains the capitalist system, which relies on an oppressed working class to generate profits », Ibid., p. 533.

787 «Relationships struck on the journey from India to Mauritius, dock marriages, the practice of hegemony and the mimicking of higher caste behaviours and practices were all experiences which the indentured labourers might have had on their travel to Mauritius [...] they were also humans who altered and attempted to change practices and beliefs in order to meet their particular needs », Ibid., p. 548.

788 «In our research we found that this was certainly the case, that Mauritians rely heavily on superficial language to assert a racial identity or categorise people in racial terms », Ibid., p. 535.

789 « Brief and commonplace daily verbal, behavioral, or environmental indignities, whether intentional or unintentional, that communicate hostile, derogatory or negative racial slights and insults towards people of colour », Ibid., p. 537.

${ }_{790}$ "In the course of research done by this team, it was found that Creoles, who are currently defined as slave descendants, routinely experienced racist attacks », Ibid., p. 592.

${ }^{791}$ «All centres provide individual, family and group counselling/psychotherapy. Some provide in house medical treatment or arrange transport for their clients to consult at the relevant hospitals. They offer holistic care in the form of occupational therapy (skills and vocational training), psychological care and spiritual care, as well as follow up/out-patient services [...] The centres accept clients from all walks of life, different ethnicity, religion and class. Although they could not provide on paper statistical data on the demographics of their clientele, the anecdotal information from Directors and Counsellors in these
} 
Comme pour les Créoles de l'île Maurice, dans Le Sari vert, Marie-Rose Patience est doublement victime. Elle est rejetée à cause de son appartenance ethnique et à cause de sa couleur. Mais en plus elle est renvoyée de son travail en raison de son lesbianisme. Son sort n'est pas exceptionnel car d'après la commission d'enquête, à l'île Maurice, ce sont les femmes noires qui souffrent le plus du racisme, et qui se retrouvent au plus bas de l'échelle sociale ${ }^{792}$.

\section{La classe sociale et le registre de langue dans Le Sari vert}

Le Sari vert brosse le tableau de la famille du docteur Bissam qui est parvenu à sortir de son milieu pauvre grâce à un dur labeur et un mariage avantageux. Le médecin a toujours eu beaucoup de clients et était submergé de travail. Marié à une femme issue d'une famille fortunée, il s'installe avec elle à Port-Louis dans une propriété dotée d'un jardin ${ }^{793}$. Or, avoir une maison et un jardin à Port-Louis est signe de richesse. Conformément à sa classe sociale, le docteur Bissam consomme de la bonne nourriture et sirote du whisky, surtout du Chivas. Sa petite-fille, Malika, est devenue institutrice et assume bien son indépendance. C'est ainsi que ce roman dépeint la classe aisée, par le biais du médecin et de sa famille mais surtout donne l'exemple de personnages qui ont travaillé dur pour améliorer leur statut social.

Tout comme dans Le Voile de Draupadi où l'avocat utilise l'anglais sur son lieu de travail et avec le médecin traitant de Wynn, le docteur Pradhan, le docteur Bissam utilisait aussi l'anglais à l'hôpital : «Os fragilisés déjà. Sans doute des fêlures, ici et là, hairline fractures, infimes mais douloureuses ${ }^{794}$. Devenu vieux, il continue à utiliser quelques mots d'anglais dans ses monologues et dans son sommeil. Occasionnellement, il se sert de l'anglais pour donner un ordre à Kitty ${ }^{795}$, ou pour dire «henpecked husband ${ }^{796}$ ou encore «sob story ${ }^{797}$. Ainsi, nous voyons comment le docteur Bissam montre une préférence pour l'anglais, tout comme les gens éduqués à l'île Maurice.

centres indicate that the majority of their clientele are Creoles of African descent with poor socio economic status, followed by Muslims », Ibid., p. 587.

792 «First, black skinned women in Mauritius experience the worst of racism. They are often the ones to be harassed and harangued. They receive the poorest levels of service. They are most discriminated against in public and government sectors », Ibid., p. 581.

793 «Je suis rentré et je les ai vues, elles, dans le jardin », Ananda Devi, Le Sari vert, Paris, Gallimard, 2009, p. 59.

${ }^{794}$ Ibid., p. 65

795 «I SAID NOW!», Ibid., p. 69.

${ }^{796}$ Ibid., p. 27

${ }^{797}$ Ibid, p. 111. 
Outre l'anglais, non traduit dans le roman, quelques mots en hindi, non traduits, tels que «daïne $»^{798}$, ou « besharam ${ }^{799}$ qui expriment la colère parsèment le texte. Il y a aussi quelques mots en créole, non traduits, dont la plupart sont des injures ou des grossièretés, tels «Bann sovaz ${ }^{800}$, «Eta Kuyon, to pa Kone ? ${ }^{801}$. Bissam utilise le créole pour exprimer sa colère : «sorti la ale zanimo ${ }^{802}$, «Lisien ! coson ! Saleté ! [...] Lisien ! coson! saleté ! lisien! saleté ! maquerelle ! zanimo ! ${ }^{803}$. Par ailleurs, l'histoire est narrée en français et à part le docteur Bissam, les personnages font toujours appel au français pour communiquer. Enfin, nous constatons que dans ce roman, les phrases en anglais, hindi et créole ne sont pas traduites, tout comme dans Le Voile de Draupadi mais contrairement au roman Rue la Poudrière.

Comme dans Le Voile de Draupadi, Le Sari vert aussi démontre l'anglais comme la langue du travail et les personnages font appel au créole pour exprimer leur colère. Par contre si dans Le Voile de Draupadi Ganeshi fait appel au hindi pour frédonner une chanson, dans Le Sari vert cette langue des ancêtres est utilisée par le médecin pour exprimer la colère.

\section{Le rêve mauricien dans Le Sari vert}

Le docteur Bissam est devenu riche et a pu intégrer la classe aisée grâce à son dur labeur, à son mariage avantageux et à son dévouement envers ses malades. D'abord étudiant diligent, ensuite médecin, il a pu réaliser le rêve mauricien et arriver en haut de l'échelle sociale, accéder au succès professionnel, acquérir du bien matériel, et surtout obtenir le respect des autres.

Bissam avait appris à travailler dur de sa mère qui travaillait sans cesse pour assurer l'avenir de son fils :

Mère, cheveux blancs dès trente ans, laide des poussières noires de la ville, de la vie accumulées dans ses rides, lourde des sédiments de désirs non exaucés, elle ne me verra pas devenir médecin. Elle est morte avant $[\ldots]$ elle

\footnotetext{
${ }^{798}$ Ce mot est utilisé aux pages 30, 61, 158, 163 du roman Le Sari vert.

799 Ananda Devi, Le Sari vert, Paris, Gallimard, 2009, p. 69.

${ }^{800}$ Ibid., p. 105.

${ }^{801}$ Ibid ; p. 128.

${ }^{802}$ Ibid., p. 152.

${ }^{803}$ Ibid., p. 134.
} 
avait laissé suffisamment d'argent de côté pour que je puisse partir ${ }^{804}$.

Grâce aux sacrifices de sa mère et aux siens, le docteur Bissam est parvenu à sortir de sa pauvreté avec dignité. Dignité dont il fait preuve quand il refuse de baisser son pantalon durant les émeutes de janvier 1968. Pour lui, ce «geste a condensé l'espoir d'une jeune nation. [Son] nom a été brandi comme le symbole d'une naissance ${ }^{805}$. Richesse, réussite sociale, dignité et reconnaissance sociale sont les valeurs auxquelles le médecin aspirait. Mais, une fois son rêve réalisé, il devient arrogant : «toujours entouré. De gens, de malades, de pauvres, de demandeurs. Moi planant au-dessus d'eux, sûr de mon utilité ${ }^{806}$. Influencé par sa mère et poussé par la société à sortir de sa condition sociale inférieure, le médecin croit fermement que la richesse et un bon statut social lui apportera puissance et bonheur. Son engouement à réaliser le rêve mauricien a un prix : sa femme meurt et il ne parvient pas à obtenir la considération de sa fille et de sa petite-fille. Ce rêve mauricien, qui est celui de beaucoup de Mauriciens, ne lui apporte ni le bonheur, ni l'amour de sa famille. Le roman, par l'entremise de la vie du docteur, critique les aspirations matérialistes de tant de Mauriciens.

\section{Relations de couples dans Le Sari vert}

Le Sari vert, tout comme Le Voile de Draupadi, met en scène trois couples, issus de trois générations différentes; le couple du docteur Bissam, celui de sa fille Kitty et celui de sa petite-fille Malika. Les deux premiers couples se sont mariés par amour. Malika et Marie-Rose s'aiment mais leur relation homosexuelle n'est socialement pas acceptée. Nous analyserons comment sont perçues leurs différences dévoilant une nouvelle tendance dans la société mauricienne et ailleurs.

\section{Le couple Bissam}

Les parents de Kitty ont fait un mariage d'amour. Ils se sont connus quand la jeune fille n'était âgée que de quinze ans et que le jeune homme était déjà médecin pratiquant. Il y a donc au moins dix ans d'écart entre les deux conjoints. La jeune fille, dont le nom n'est jamais donné, est issue d'une famille riche tandis que le jeune homme est pauvre. La relation a débuté sans le consentement des parents de la jeune fille, qui finissent par accepter le mariage. Très rapidement, il y a des scènes de violence, où le

\footnotetext{
${ }^{804}$ Ibid., p. 42.

${ }^{805}$ Ibid., p. 131.

${ }^{806}$ Ibid., p. 209.
} 
mari laisse libre cours à sa colère envers sa femme. Cette violence va en augmentant et mène à la mort de l'épouse. Pour le médecin, la violence est un exutoire qui le soulage et le libère des tensions accummulées :

Je dois avouer qu'à ce tout premier acte que je dois par honnêteté qualifier de violent il y a en moi comme une éruption d'un bonheur triomphal, si riche et si chaud que cela m'est tout de suite monté à la tête. Je me suis senti libéré de tout un poids de faux semblants. Toute la tension que je ressentais s'est dissipée ${ }^{807}$.

Son épouse subit en silence ses assauts sans jamais protester : «après le premier cri de surprise elle n'avait plus crié ni protesté ${ }^{808}$. Le médecin estime que « ce qui n'était pas normal, c'était qu'elle refuse, elle, l'ordre établi et s'engage dans cette guerre silencieuse contre moi. C'est cela qui nous a conduits à la destruction ${ }^{809}$.

$\mathrm{Au}$ début, le médecin supporte mal que sa femme ne soit pas une bonne cuisinière. Après la naissance de Kitty, il accorde à son épouse un certain temps «pour nourrir, changer, baigner, bercer la petite, lui chanter des chansons, lui raconter des histoires, la câliner ${ }^{810}$ en espérant qu'elle reprenne vite ses responsabilités de ménagère et de maîtresse de maison. Lorsque ceci ne se produit pas, il passe à un deuxième degré de violence et décide de se servir de son scalpel pour lui apprendre sa leçon : «pour bien faire pénétrer ce message, j’ai appuyé un tout petit peu sur la lame et j'ai vu avec une joie inouïe perler des gouttes de sang ${ }^{811}$. Selon le docteur, sa femme, «n'a pas compris que je faisais cela pour sauver ce qu'il restait de notre famille $\gg{ }^{812}$.

De plus en plus passive, sa femme meurt ébouillantée et brûlée alors que Kitty est âgée d'à peu près deux ans. Les circonstances exactes de cette mort restent énigmatiques. D'après les dires du médecin, c'est Kitty qui avait eu l'idée d'immoler sa mère en disant : «tu sais comment on punit les sorcières ? [...] il faut les brûler » ${ }^{813}$. Il se rappelle avoir été troublé par ces paroles de Kitty mais pense que Kitty est coupable :

\footnotetext{
${ }^{807}$ Ibid., p. 28.

${ }^{808}$ Ibid.

${ }^{809}$ Ibid., p. 24.

${ }^{810}$ Ibid., p. 57.

${ }^{811}$ Ibid., p. 60

812 Ibid.

${ }^{813}$ Ibid., p. 187.
} 
Ma main trenble. J'allume une cigarette. Ma main tremble. J'aspire une bouffée qui me brûle le cœur. Ma main tremble. Je secoue l'allumette pour l'éteindre et la jette à terre. Ma main tremble. Je pose la boîte d'allumettes et la boîte de cigarettes sur la table.

Ne joue pas avec les allumettes, Kitty, dis-je en sortant de la pièce.

Kitty, je t'ai aimée après la mort de ta mère, mais je n'ai pu oublier ce que tu as fait ${ }^{814}$.

Pour sa part, Kitty, devenue adulte, accuse son père d'avoir tué sa mère, d'abord en jetant du riz chaud sur sa tête et ensuite en jetant l'allumette sur son sari :

Je me souviens de son visage au moment précis où tu as renversé la marmite sur sa tête [...] Elle était prête. Je crois qu'elle était déjà morte quand le feu a pris [...] Heureusement pour elle, elle est partie avant que tu ne jettes ton allumette encore enflammée sur le pan de son $\operatorname{sari}^{815}$.

Ce qui est sûr, c'est que la mère de Kitty n'a réagi à aucun moment et s'est en quelque sorte laissée mourir :

Tu ne la regardais pas comme moi. Moi, j'avais les yeux fixés sur les siens, et j'ai vu clairement l'instant précis où la lumière est partie et il n'y avait plus rien là, que le corps de ma mère; ni sorcière ni mère, seulement une femme fracassée. Je n'y comprenais rien mais j'ai su qu'elle était partie ${ }^{816}$.

Pour Durkheim, « le meurtrier et le suicidé seraient tous deux des dégénérés et des impuissants. Egalement incapables de jouer un rôle utile dans la société, ils

${ }^{814}$ Ibid., p. 187-188.

${ }^{815}$ Ibid., p. 200.

${ }^{816}$ Ibid. 
seraient, par [la] suite, destinés à être vaincus ${ }^{817}$. Le caractère violent, misogyne et narcissique du médecin relèverait de son enfance vécue dans la misère et la faim, et du fait qu'il a dû survivre seul contre tous. Durkheim explique que quand : «l'existence humaine est moins respectée, [l'individu] se révoltera, déclarera la guerre à la société, tuera au lieu de se tuer ${ }^{818}$. Pendant cinq ans de mariage, l'épouse de Bissam a subi des atrocités de la part de son mari, et à aucun moment le roman ne mentionne l'intervention des parents de la jeune femme pour mettre un terme à cette violence. Dans un entretien donné au journal quotidien mauricien, l'avocate mauricienne Rada Gungaloo affirme que «dans les quartiers aisés, la femme a honte de dénoncer à cause de son statut et celui de son mari ${ }^{819}$. Sans limites, les atrocités ont continué et la violence conjugale a vite progressé à l'homicide. Comme le remarque Durkheim: « tantôt la violence d'où ils dérivent, ne rencontrant pas de résistance dans le milieu social, s'y répand, et alors, elle devient homicide ${ }^{820}$. Il est possible que la jeune femme ait préféré se taire, d'autant plus que les femmes hindoues sont éduquées pour servir le mari sans se plaindre ni se rebeller.

Mais si d'une part l'épouse n'a jamais pu satisfaire les attentes de son mari, celui-ci n'a pas pu accepter que sa jeune épouse, qui attirait l'attention de tout le monde par sa beauté, ne soit pas le reflet de sa mère à lui. Donc, pour que sa femme marche sur les pas de sa mère, il a recours à la violence puisqu'il avoue que «j'ai dû lui apprendre avec des coups de poing $»^{821}$. Il reproche en particulier à sa femme de ne pas être une bonne cuisinière, comme l'était sa mère qui lui : «préparait des gâteaux-patate avec les tubercules [...] aujourd'hui j'y pense et l'eau me vient à la bouche [...] le chant sucré de mon enfance ${ }^{822}$. Le mari n'a jamais réalisé que sa femme, mariée à quinze ans, n'était en effet qu'une adolescente, ayant grandi dans la richesse et le confort. Ces explications et suppositions ne sont pas des raisons qui légitimisent l'extrême violence du mari, mais d'après Merton le 'ressentiment' pourrait expliquer les actions du docteur. En effet, une personne rongée par ce sentiment est submergée

\footnotetext{
${ }^{817}$ Emile Durkheim, Le Suicide. Etude de sociologie. Livre troisième. Du Suicide comme phénomène social en général, http://classiques.uqac.ca/classiques/Durkheim_emile/suicide/suicide_Livre_3.pdf, consulté le 9 janvier 2014, p. 42.

818 Ibid.

${ }^{819}$ Jane Lutchmaya, «Violence domestique : quand la répression ne suffit pas ! », http://www.defimedia.info/defi-quotidien/dq-actualites/item/49078-violence-domestique-quand-la repression-ne-suffit-pas.html, consulté le 21 mars 2014.

${ }^{820}$ Emile Durkheim, Le Suicide. Etude de sociologie. Livre troisième. Du Suicide comme phénomène social en général, http://classiques.uqac.ca/classiques/Durkheim_emile/suicide/suicide_Livre 3.pdf, consulté le 9 janvier 2014, p. 42.

${ }^{821}$ Ananda Devi, Le Sari vert, Paris, Gallimard, 2009, p. 25.

${ }^{822}$ Ibid., p. 81.
} 
par la haine ou l'hostilité ${ }^{823}$ et réagit violemment car elle se voit dans l'incapacité d'atteindre ce qu'elle désire ${ }^{824}$; tout comme le médecin qui désirait une femme, à l'image de sa mère mais se voit dans l'impossibilité de réaliser ce désir.

D’après un journal quotidien mauricien, au moins cinq femmes ont été tuées d'une façon atroce de janvier à mars $2014^{825}$, et selon l'étude menée par Gender Links en 2012, à Maurice, « une femme sur quatre est victime de violence domestique ${ }^{826}$. De plus, d'après Rada Gungaloo, contrairement aux préjugés, les cas de violence domestique touchent «autant les couches sociales aisées que les quartiers populaires $»^{827}$. La violence de Bissam vis-à-vis de sa femme est à l'image de toute la société patriarcale mauricienne. D’après Ibrahim Koodoruth, sociologue mauricien, « la violence envers les femmes est aussi une affaire d'hommes ${ }^{828}$. La solution à ce fléau serait, d'après Jeanne Ambal, directrice de SOS Femmes, « que la société mauricienne ne soit plus patriarcale et machiste $! »^{829}$.

Ainsi les cas de violence à l'île Maurice n'englobent pas que la communauté aisée hindoue mais toutes les couches de la société. Cette violence touche aussi de nombreux pays. Par exemple en Inde, le nombre de femmes tuées par leur mari est en nette hausse ${ }^{830}$ et nous notons que beaucoup de ces femmes meurent brûlées, comme la femme du docteur. Selon Virendra Kumar, 40 pour cent des femmes en Inde sont tuées à l'aide d'allumettes, et 21,87 pour cent sont brûlées pendant les cinq premières années de leur mariage, comme pour la femme du docteur Bissam. De plus, 25 pour cent sont

${ }^{823}$ «First, diffuse feelings of hate, envy and hostility», Robert Merton, Social Theory \& Social Structure, New York, The Free Press, 1968, p. 210.

824 «Ressentiment [...] asserts merely that desired but unattainable objectives do not actually embody the prized value », Robert Merton, Social Theory \& Social Structure, New York, The Free Press, 1968, p. 210.

${ }^{825}$ Melanie Valere, « Dossier "Femmes". Les humains s'accordent aussi au féminin », http://www.defimedia.info/defi-quotidien/dq-societe/item/49252-dossier-femmes-les-droits-humains-saccordent-aussi-au-feminin.html, consulté le 21 mars 2014.

${ }^{826}$ Jane Lutchmaya, «Violence domestique : quand la répression ne suffit pas !», http://www.defimedia.info/defi-quotidien/dq-actualites/item/49078-violence-domestique-quand-larepression-ne-suffit-pas.html, consulté le 21 mars 2014.

${ }_{827}$ Ibid.

${ }^{828}$ Melanie Valere, « Dossier "Femmes". Les humains s'accordent aussi au féminin », http://www.defimedia.info/defi-quotidien/dq-societe/item/49252-dossier-femmes-les-droits-humains-saccordent-aussi-au-feminin.html, consulté le 21 mars 2014.

829 Jane Lutchmaya, «Violence domestique : quand la répression ne suffit pas ! », http://www.defimedia.info/defi-quotidien/dq-actualites/item/49078-violence-domestique-quand-larepression-ne-suffit-pas.html, consulté le 21 mars 2014.

$\frac{8}{830}$ «Female homicide is one of the leading causes of injury deaths in women $[\ldots]$ The victims were most frequently killed by their spouses. Argument (33.3\%) and dowry (30.7 \%) were most frequent reasons behind the crime. Majority of victims $(84.6 \%)$ were killed in their home. Fatal wound is most frequently seen in head, and defense wound is present only in nine cases. Considering sociological aspect of victim, $82 \%$ of the victims were from Hindu religion », Manoj Mohanty, Manoj Panigrahi, Sachidananda Mohanty, Srimanta Das, «Victimiologic Study of Female Homicide», Legal Medecine, 6, 2004, p. 151 
mortes instantanément de leurs brûlures ${ }^{831}$ et 80 pour cent de ces actes de barbarie sont commis dans la maison du couple ${ }^{832}$, exactement comme la femme du docteur. Et de toutes ces morts par le feu, 93,76 pour cent proviennent de familles hindoues ${ }^{833}$. Aussi, la mort de la mère de Kitty est-elle représentative d'une société patriarcale qui trouve ses sources en Inde.

\section{Kitty et son mari}

Contrairement au couple désuni et violent de ses parents, Kitty et son mari ont une excellente relation. Si le couple ne connaît que le bonheur et les joies de la vie conjugale, le mari de Kitty meurt « de tuberculose cinq ans après leur mariage ${ }^{834}$. Le mari de Kitty est issu d'une famille hindoue, de classe supérieure car le médecin a donné son aval : «la demande a été faite en bonne et due forme ${ }^{835}$ par la famille du futur mari.

Kitty était tombée amoureuse de ce bibliothécaire, et craignant une réaction négative, ne parle à son père de sa relation que bien plus tard : «ce n'est que lorsque tout a été décidé et les fiançailles célébrées que j'ai su qu'ils avaient tout manigancé, lui et Kitty ${ }^{836}$. Le mari de Kitty est doux, bon ${ }^{837}$, calme, timide et intelligent. Le vieux médecin pense que «Kitty l'avait choisi parce qu'il était [son] contraire ${ }^{838}$. A la mort de son mari, Kitty et sa fille sont allées vivre chez le médecin à Port-Louis. Une fois chez son mari, « Kitty a voulu couper les ponts ${ }^{839}$ avec son père mais après la mort de son mari, la jeune mère seule et veuve désorientée retourne à la maison paternelle. Mais elle réalise très vite qu'elle ne peut plus rester sous le même toit et décide d'aller vivre loin de son père, avec sa fille, à Curepipe ${ }^{840}$.

Le mariage de Kitty, comme celui de ses parents, n'a duré que cinq ans. Mais, en optant pour un mari qui est le contraire de son père et en fuyant la maison parentale après la mort de son mari, Kitty montre qu'elle ne peut plus tolérer la violence de son père. Contrairement à sa mère, elle se rebelle, et dans les termes de Merton, elle rejette

\footnotetext{
${ }^{831}$ Virendra Kumar, « Burnt Wives-a Study of Suicides », Burns, 29, 2003, p. 32.

${ }^{832}$ Ibid., p. 33

${ }^{833}$ Ibid.

${ }^{834}$ Ananda Devi, Le Sari vert, Paris, Gallimard, 2009, p. 139.

835 Ibid., p. 138.

${ }^{836}$ Ibid.

${ }^{837}$ «C'était un homme bon », Ibid., p. 139.

${ }^{838}$ Ibid., p. 142.

${ }^{839}$ Ibid., p. 146.

840 « J'ai pris ma place naturelle. Elles me sont revenues, toutes les deux [...] jusqu'à ce que Kitty finisse par prendre la fuite et se sépare de moi », Ibid.
} 
les valeurs qu'elle avait jusque-là respectées ${ }^{841}$. En effet, d'après les anciennes traditions hindoues, la veuve devait être brûlée sur le bûcher funéraire de son mari. Avec le temps, cette pratique, considérée comme inacceptable, a cessé mais est toujours occasionnellement observée en Inde ${ }^{842}$. Depuis la cessation du "sati”, les veuves sont rejetées en Inde car elles sont considérées comme porteuses de malheur. À l'île Maurice, si la veuve n'est pas brûlée vivante ou rejetée par sa belle-famille, elle est considérée comme une commodité et revient souvent à la charge du père. Il serait presqu' impossible pour une jeune veuve comme Kitty de survivre seule, sans emploi, et ayant à charge un enfant avant que le gouvernement mette en place les pensions de veuves en 1969. La décision de Kitty de mener une vie indépendante reflète donc une évolution sociale vis-à-vis des femmes à l'île Maurice.

\section{Malika et Marie-Rose Patience}

Malika et Marie-Rose Patience vivent en concubinage et font un couple mixte. Le roman donne peu de détails sur la vie de Marie-Rose, mais nous savons que les deux jeunes femmes ont été renvoyées de leur travail à cause de leur préférence sexuelle et qu'elles se retrouvent au chômage : « on nous a virées toutes les deux dès que la chose a été sue ${ }^{843}$.

Malika affiche ouvertement sa relation homosexuelle que son grand-père condamne et que sa mère, Kitty, semble accepter. Aussi, le roman expose deux points de vue radicalement différents sur le lesbianisme, venant de deux générations différentes. Malika, en racontant ses rapports sexuels, provoque délibérément son grand-père, pour qui le lesbianisme est inacceptable. Si Malika démontre sa préférence sexuelle, c'est peut-être pour se protéger contre les hommes, vu qu'elle connaît la souffrance de sa mère et la fin tragique de sa grand-mère. Le médecin considère que ce genre de relation est honteux : «comme ça a dû lui faire mal à Kitty, comme ça a dû la ronger et la faire saigner de honte par tous les pores ${ }^{844}$. Tout comme lui, nombreux sont les Mauriciens qui sont submergés par la honte lorsqu'ils apprennent que leur

\footnotetext{
841 «Involves a genuine transvaluation, where the direct or vicarious experience of frustration leads to full denunciation of previously prized values », Robert Merton, Social Theory \& Social Structure, New York, The Free Press, 1968, p. 210.

${ }^{842}$ Kavita Srivastava, general secretary of the People's Union for Civil Liberties, Rajasthan, has been actively working to discourage the practice since 1987. She says "the law has been very effective in preventing the making of a sati" but adds that "it has not been equally successful in preventing the glorification of sati even though it has been rendered a crime" », A. Divya, "Why Sati is still a burning issue », The Times of India, http://timesofindia.indiatimes.com/home/stoi/Why-sati-is-still-a-burningissue/articleshow/4897797.cms?, consulté le 12 août 2014.

${ }_{843}$ Ananda Devi, Le Sari vert, Paris, Gallimard, 2009, p. 46.

${ }^{844}$ Ibid.
} 
enfant est homosexuel et n'hésitent pas à le séquestrer et le torturer pour le guérir ou lui faire changer de comportement ${ }^{845}$. La perception du grand-père représente celle de toute une génération qui n'est pas prête à accepter l'homosexualité, est traditionellement répréhensible : «maintenant elles préfèrent se tourner vers la femme et se passer des hommes, qui eux se tournent vers d'autres hommes ${ }^{846}$. Pour lui, le rôle principal de la femme est d'enfanter : «la plupart des hommes de ma génération avaient plusieurs femmes et une portée d'enfants naturels qui tombaient comme des blattes et que les épouses légitimes devaient accepter ${ }^{847}$. Pour lui qui a toujours eu « un comportement normal ${ }^{848}$, sa petite-fille est malade. Mais à croire un quodidien mauricien, même en 2014, il y aurait des hommes, dans la société patriarcale mauricienne qui partagent l'avis du vieux médecin. Ainsi, d'après le sociologue Surendr Nowbuth :

A Maurice, il y a une culture ancestrale qui veut que la femme ne travaille pas, mais qu'elle reste à la maison pour s'occuper de sa famille et du ménage [...] Outre cet esprit conservateur, le second facteur qui pousse les hommes à préférer à ce que leur femme reste à la maison, c'est la jalousie [...] il y a encore d'autres maris qui, occupant déjà un travail bien rémunéré, ne trouvent pas nécessaire que leur femme travaille. Ils préfèrent la voir rester à la maison pour s'assurer que leur enfant a toute l'affection maternelle nécessaire, grandisse dans un bon cadre $^{849}$

Comme le montre l'analyse faite en 2011 par le Committee on the Elimination of Discrimination against Women, la société mauricienne reste stéréotypée, l'homme étant toujours considéré comme le principal gagne-pain et la femme ayant la charge des

\footnotetext{
845 «In the Indian Ocean of Mauritius, sodomy is prohibited and society is intolerant of gay and lesbian relationships. We've already had a case where a young girl was abducted on the beach by her parents and confined [...], or where a young girl was locked up in a psychiatric hospital and physically threatened by her family. But for every one who has the courage to testify, how many cases are kept quiet? Asks Natalie Ahnee, President of the Collectif Arc-en-Ciel », LGBT Asylum News, "Gays in Mauritius are kidnapped to "cure" their homosexuality ", http://sdgln.com/causes/2010/08/25/gays-mauritius-arekidnapped-cure-their-homosexuality\#sthash.ptF5ObuT.dndyNnUw.dpbs, consulté le 23 mars 2014.

${ }_{846}$ Ananda Devi, Le Sari vert, Paris, Gallimard, 2009, p. 51.

${ }^{847}$ Ibid, p. 50.

${ }^{848}$ Ibid, p. 51

849 Raj Bissessur, «Chômage. Quand la femme est victime», http://www.defimedia.info/defiquotidien/dq-societe/item/57712-chomage-quand-la-femme-est-victime.html, consulté le 13 août 2014.
} 
enfants ainsi que des tâches ménagères ${ }^{850}$. L'attitude du vieil homme est certainement partagée par beaucoup d'autres à l'île Maurice qui n'hésitent pas à enfermer leur fille à l'asile afin de la guérir de cette maladie qu'est le lesbianisme ${ }^{851}$. Pour sa part, la loi n'accepte ni ne pénalise l'homosexualité à l'île Maurice ${ }^{852}$ mais elle pénalise la sodomie, même parmi les hétérosexuels ${ }^{853}$.

A vrai dire, d'après les statistiques présentées par l'Amnesty International, l'île Maurice tombe dans la catégorie des pays africains où l'homosexualité est toujours illégale mais en 2009 , le gouvernement mauricien a décidé de la décriminaliser ${ }^{854}$ montrant une ouverture d'esprit nouvelle, comme la mère de Malika qui ne semble pas avoir d'objection à l'homosexualité de sa fille. Kitty représente une génération qui est plus tolérante que la précédente. A travers le cas de Malika et Marie-Rose, le roman montre que l'homosexualité commence à être acceptée par certains dans la société mauricienne. Pour Linley Couronne d'Amnesty Mauritius, les marches pacifiques annuelles des homosexuels à l'île Maurice, sont un grand pas vers l'acceptation ${ }^{855}$ et pour Natalie Ahnee, présidente de Collectif Arc-en Ciel

L'homosexualité est acceptée par certains, et tolérée par d'autres, il reste des gens pour qui les homosexuels,

850 «The Committee is deply concerned about the persistence of discriminatory cultural norms and practices as well as patriarchal attitudes and deep rooted stereotypes concerning the roles and responsibilities of women and men in family and society whereby men, at large, are still considered the main breadwinners and women are considered as having the primary responsibility for child-rearing and domestic tasks », Convention on the Elimination of All Forms of Discrimination against Women, «Concluding observations of the Committee on the Elimination of Discrimination against Women: Mauritius »,

http://www2.ohchr.org/english/bodies/cedaw/docs/co/CEDAW-C-MUS-CO-6-7.pdf, consulté le 25 mars 2014, p. 4.

${ }^{851}$ Agenda News, « Mauritius. Trauma for Young Lesbians », http://www.afrika.no/Detailed/12586.html, consulté le 24 mars 2014.

852 Ibid.

${ }^{853}$ Bureau of Democracy, Human Rights and Labour, « 2013 Human Reports Rights: Mauritius », http://www.state.gov/j/drl/rls/hrrpt/2013/af/220139.htm, consulté le 24 mars 2014.

${ }^{854}$ «On 10 February 2009, Mauritius committed to finalizing and adopting its Sexual Offences Bill, which would have the effect of decriminalizing homosexuality », Amnesty International, « Making Love a Crime. Criminalization of Same-Sex Conduct in Sub-Saharan African », http://www.amnesty.org/en/library/asset/AFR01/001/2013/en/9f2d91b7-bc0e-4ea7-adae7e51ae0ce36f/afr010012013en.pdf, consulté le 24 mars 2014, p. 17.

855 «Linley Couronne of Amnesty Mauritius mentions that there has been some evolution in the mentality as evidence by recent Gay Pride, an annual march in support of the rights of hemosexuals », SADC Gender Protocol, « Mauritius. Homophobia : One Step Forward, Two Steps Back », http://www.genderlinks.org.za/article/mauritius---homophobia-one-step-forward-two-steps-back-201106-17, consulté le 25 mars 2013. 
lesbiennes et ceux qui ne sont pas hétérosexuels, sont une plaie, une maladie ${ }^{856}$.

Il est à noter que la toute première marche pacifique pour les droits des homosexuels, aussi connu comme Gay Pride de l'île Maurice a eu lieu le 20 mai $2005^{857}$. Victimes de discriminations, Malika et Marie-Rose ont dû démissionner faute de loi antidiscriminatoires, loi qui n'a été adoptée à l'île Maurice qu'en $2008^{858}$ quand l'association Collectif Arc-en Ciel a réussi à faire inclure dans l'Equal Opportunites Act «toutes formes de discrimination homosexuelle ${ }^{859}$. Outre la question du lesbianisme comme tabou à l'île Maurice, ce roman fait aussi référence, à travers Malika et MarieRose, au taux de chômage de la femme mauricienne.Ainsi, « selon le dernier rapport de Gender Statistics pour le troisième trimetres de 2013, le taux de chômage était de 7,8 pour cent. Des 47300 chômeurs enregistrés, 19700 (42 pour cent) étaient des hommes et 27600 (58 pour cent) étaient des femmes [et] près de 60 pour cent des chômeurs à Maurice étaient des femmes ${ }^{860}$. De plus, beaucoup de femmes « auparavant actives sur le plan professionnel abandonnent leur emploi pour cause [d'être] licenciées » ${ }^{861}$, tout comme Malika et Marie-Rose.

\section{Représentation réaliste à la fois locale et universelle des ethnies et classes sociales dans Le Sari vert}

Le Sari vert fait la lumière sur les discriminations sociales qui existent contre les castes inférieures dans la communauté indo-mauricienne, et contre les homosexuels et contre les femmes, par l'entremise de la famille du docteur Bissam qui subit de façon différente le poids des traditions hindoues et des valeurs patriarcales. Le roman décrit

\footnotetext{
${ }^{856}$ Jenilaine Moonean, « Droits de la communauté LGBT. A la recherche du temps perdu », http://www.defimedia.info/defi-quotidien/dq-societe/item/9995-droits-de-la-communaut\%C3\%A9-lgbt\%E2\%80\%93-\%C3\%A0-la-recherche-du-temps-perdu.html, consulté le 07 juin 2014. explicitly outlaw discrimination on the basis of sexual orientation, or they have removed discriminatory provisions of existing employment legislation [...] and Mauritius in 2008 », « Making Love a Crime: Criminalization of Same-Sex Conduct in Sub-Saharan African », http://www.amnesty.org/en/library/asset/AFR01/001/2013/en/9f2d91b7-bc0e-4ea7-adae7e51ae0ce36f/afr010012013en.pdf, consulté le 24 mars 2014, p. 18.

${ }_{859}$ Jenilaine Moonean, « Droits de la communauté LGBT. A la recherche du temps perdu », http://www.defimedia.info/defi-quotidien/dq-societe/item/9995-droits-de-la-communaut\%C3\%A9-lgbt\%E2\%80\%93-\%C3\%A0-la-recherche-du-temps-perdu.html, consulté le 07 juin 2014.

860 Raj Bissessur, «Chômage. Qaund la femme est victime », http://www.defimedia.info/defiquotidien/dq-societe/item/57712-chomage-quand-la-femme-est-victime.html, consulté le 13 août 2014 ${ }^{861}$ Ibid.
} 
de façon très réaliste comment ces valeurs ancestrales changent avec chaque génération.

\section{Réalisme social et thèmes sociaux universels}

Dans ce chapitre, l'analyse de la dimension sociale dans les trois romans a permis d'étudier de près la représentation de la société mauricienne et d'en évaluer le réalisme et la portée universelle des thèmes.

Rue la Poudrière, qui traite surtout de la communauté créole et de sa situation précaire dans les années 1959 à 1979 en décrivant la vie de Paule et de sa famille à Port-Louis, donne des descriptions peu flatteuses des personnages créoles et de leurs conditions et reflète, à en croire le quatrième rapport de la Truth and Justice Commission, un fléau bien réel qui affectait la communauté créole de Maurice. La langue créole utilisée pour communiquer est perçue comme un manque d'éducation qui caractérise cette communauté pauvre dans les années où se déroule l'histoire. La perception et la description des Créoles et de leur mode de vie faite par l'auteure reflète très justement le sort misérable de ce groupe ethnique en bas de l'échelle sociale. A travers les problèmes sociaux soulevés tels l'alcoolisme, le chômage, la violence et la prostitution, le roman dénonce des dysfonctions sociales réelles qui caractérisaient l'époque pré-indépendante encore marquée par le passé colonial.

Dans Le Voile de Draupadi, l'accent est mis sur les communautés hindoue et musulmane ainsi que sur la relation tendue entre les communautés hindoue et créole par l'entremise de la famille d'Anjali et de Faisal, qui vivent à Port-Louis dans les années en 1986. Ce roman touche un point bien réel de la société mauricienne en démontrant la ségrégation raciale des Créoles que le rapport d'enquête de la Truth and Justice Commission a prouvée. Outre le racisme, cette commission d'enquête démontre aussi à quel point la communauté indo-mauricienne est influencée par la notion de caste si importante pour le grand-père et la mère d'Anjali. Le Voile de Draupadi dépeint en effet une classe aisée qui détient le pouvoir monétaire, qui utilise l'anglais comme langue de prestige, et explore les effets du mariage arrangé et des coutumes ancestrales hindoues dans la société mauricienne. Par le biais de la relation entre Shyam et Margaret, le roman met à jour une certaine ouverture de la société mauricienne en ce qui concerne les unions mixtes. 
Le Sari vert dépeint une famille hindoue aisée très dysfonctionnelle habitant Port-Louis et Curepipe, dans la première moitié des années 1980, dont le père de famille, le docteur Bissam, est respecté dans la société mais violent envers sa jeune femme. La description de la la violence, des mariages arrangés et des traditions hindoues et de l'impact du rêve mauricien font écho à la réalité mauricienne. La situation de femme battue de la mère de Kitty et sa mort montre un fléau qui vient de l'Inde et ronge la société mauricienne. En plus, la question du lesbianisme est aussi abordée dans le roman et révèle qu'il est peu accepté dans la société mauricienne malgré une certaine évolution dans les attitudes des plus jeunes générations.

Tout en présentant des phénomènes sociaux qui touchent profondément la société mauricienne, les trois romans à l'étude dépassent les frontières de cette île pour aborder des sujets universels. Les questions de pauvreté, de chômage, de prostitution touchent la plupart des pays en voie de développement où la classe inférieure fait face aux mêmes maux sociaux et où les plus démunis sont prêts à adopter des moyens illégitimes pour assurer leur survie. La situation inférieure du Créole mauricien est comparable à celle des Noirs, à en croire Paul François : «la situation des Noirs américains, ressemble quelque peu à celle des Créoles mauriciens ${ }^{862}$. Juliet Hooker, elle, avance qu'au Brésil 60 pour cent des Afro-Brésiliens sont pauvres ${ }^{863}$ et que les Noirs de l'Amérique latine souffrent aussi de discrimination raciale sur le marché du travail $^{864}$, tout comme en Colombie, en Ecuador, à Honduras, et au Nicaragua où la population noire rurale a longtemps lutté pour ses droits, pour le contrôle des ressources naturelles, l'autonomie territoriale ou politique et la reconnaissance culturelle ${ }^{865}$. La marginalisation des descendants des esclaves noirs de l'île Maurice semble générale. De plus, le comportement du proxénète Mallacre qui amadoue ses proies pour mieux les exploiter et du client qui tombe amoureux de la prostituée est un phénomène bien connu des spécialistes qui notent les mêmes pratiques un peu partout dans le monde.

Les questions de castes, et de disparités raciales, le complexe de supériorité et d'infériorité ne sont pas uniques à l'île Maurice non plus. En Inde, le système de caste renforce les complexes de supériorité et d'infériorité, et le racisme lié à la couleur de la

\footnotetext{
${ }^{862}$ Paul François, «La Communauté créole de 1'île Maurice », http://csmsmagazine.org/?p=1667, consulté le 29 octobre 2013.

863 «In Brazil 60 percent of Afro-Brazilians are poor », Juliet Hooker, «Indigenous Inclusion/Black Exclusion: Race, Ethnicity and Multicultural Citizenship in Latin America », Journal of Latin American Studies, 37, 2005, p. 288.

864 « Blacks in Latin America also suffer racial discrimination in the labour market », Ibid.

865 «Rural black populations have struggled for rights to command land, control over natural resources, territorial or political autonomy, and cultural recognition », Ibid., p. 295.
} 
peau. La violence et le meurtre au sein de la famille sont fréquents en Inde, où la femme est jugée faible par l'homme. De fait, d'après les chiffres mis en avant par la World Health Organisation, de toutes les femmes tuées, 38 pour cent le sont par leurs conjoints $^{866}$, tout comme la femme du médecin dans Le Sari vert et 16 pour cent de ces femmes mettent au monde des bébés faibles et chétifs à la naissance. Le rejet du lesbianisme est typique de toutes les sociétés traditionnelles, comme l'Inde où les lesbiennes ne trouvent pas leur place. Ainsi, comme Malika et Marie-Rose qui ont dû quitter leur emploi de force, leur emploi, de nombreuses lesbiennes en Inde font face au même sort : d'après une recherche faite sur la violence contre les femmes en Inde, il est clair que les lesbiennes sont forcées de quitter leur logement ou leur travail ${ }^{867}$.

De ce fait, nous pouvons affirmer que dans Rue la Poudrière, Le Voile de Draupadi et Le Sari vert, Ananda Devi décrit avec réalisme les problèmes sociaux qui affectent la société mauricienne, maux sociaux universels qui se retrouvent ailleurs dans le monde. En décrivant la situation sociale de l'île Maurice, Ananda Devi aborde des thèmes universels et dénonce les injustices sociales, la violence contre les femmes et les préjugés raciaux.

\footnotetext{
${ }^{866}$ World Health Organization, « Global and Regional Estimates of Violence against Women: Prevalence and Health Effects of Intimate Partner Violence and Non-Partner Sexual Violence. 2013 », http://apps.who.int/iris/bitstream/10665/85239/1/9789241564625_eng.pdf, consulté le 21 mars 2014, p. 2.

${ }^{867}$ « There have been instances of lesbians being thrown out of their rental accommodation, or out of their jobs, when landlords or employers have found out about their sexual orientation », Bina Fernandez, N.B. Gomathy, « The nature of violence faced by lesbian women in India », http://download.tiss.edu/fap/RCI-VAW/RCIVAW Publications/The Nature of violence faced by Lesbian_women in_India.pdf, consulté le 21 mars 2014, p. 10.
} 


\section{Partie V}

\section{Du local à l'universel : trois romans réalistes}




\section{Récapitulation}

L'analyse des dimensions spatiale, temporelle et sociale des trois romans de Devi a permis de voir à quel point ceux-ci dépeignent la société mauricienne de façon authentique, mais aussi à quel point les questions sociales abordées sont à la fois locales et universelles.

L'étude de la dimension spatiale a montré que Devi utilise une toponymie et une topographie bien mauricienne dans les trois romans dont l'histoire est située soit à Port-Louis, soit dans des villes et villages du centre et du sud de l'île. En fait, cette localisation spécifique est indéniable dans Le Voile de Draupadi et Le Sari vert mais moins marqué dans Rue la Poudrière. Ceci relève surtout de l'exigence des éditeurs, comme l'explique Devi durant une de ses entrevues : "Quand j'ai envoyé Rue la Poudrière aux éditeurs parisiens, le manuscrit a été systématiquement refusé parce qu'il “manquait de couleur locale ". J'ai compris que, du fait d'être Mauricienne, on exigeait de moi une écriture exotique ${ }^{868}$. Nous sommes donc en droit de nous demander si, après la déception concernant Rue la Poudrière, l'auteure a voulu répondre aux attentes des éditeurs en accentuant plus le caractère local des romans qui ont suivi, comme $L e$ Voile de Draupadi et Le Sari vert. Devi explique :

Je me rappelle que ces refus ont été très durs pour moi [...] Suite aux refus successifs des éditeurs au début de ma carrière d'écrivain, je me suis fortement remise en question, et pendant plusieurs années, j'ai cherché ma voix d'écrivain. J'ai l'impression que ce questionnement est visible si l'on compare le style de Rue la Poudrière, qui est un texte écrit d'un jet et à la première personne, avec celui du Voile de Draupadi, dont le lyrisme est beaucoup plus contenu et qui est moins spontané ${ }^{869}$.

De fait, dans Rue la Poudrière, si les lieux de Maurice sont expressément nommés, leur description s'avère plutôt universelle, et la flore, la faune, les insectes ou reptiles cités ne sont pas nécessairement locaux et renvoient à l'ailleurs ou à l'universalité des mythes. Tout le roman, centré sur la ville de Port-Louis, « restitue une perception

${ }^{868}$ Mar Garcia, «Entretien avec Ananda Devi »La Tortue verte : revue en ligne des littératures francophones, http://www.latortueverte.com/Entretien\%20avec\%20Ananda\%20Devi.pdf, consulté le 5 septembre 2011, p. 4.

${ }^{869}$ Ibid., p. 4, 5. 
diachronique de la réalité ${ }^{870}$ mauricienne dans la mesure où le lecteur découvre les changements économiques aussi bien que sociaux de cette ville et de ses habitants. La ville agit comme un «marquage symbolique [...] qui tisse une trame de lieux de mémoire ${ }^{871}$. De cette manière, le lecteur mauricien ou étranger est en mesure d'appréhender le monde de Devi qui met « la fiction [...] au service de la réalité » ${ }^{872}$ pour ancrer son histoire à Port-Louis, un lieu réel, tout en créant des personnages et un univers aux allures plus universelles. Par contre, Le Voile de Draupadi mentionne le nom de plusieurs endroits de balades ou d'escalade connus de Maurice et Le Sari vert fait mention d'endroits symboliques et significatifs pour l'île; Le Morne en est le parfait exemple. Le Sari vert fait référence à des villes et villages spécifique de l'île et le narrateur précise bien qu'il se considère comme Mauricien: «je suis devenu l'exemple du vrai Mauricien ${ }^{873}$. Nous lisons encore, « un whisky qui n'existait pas encore à Maurice, que personne n'avait encore jamais goûté ici » ${ }^{874}$, ou encore «moi, étudiant brillant à Dublin, moi rentré, triomphant à Maurice » ${ }^{875}$. Cet ancrage toponymique se double de descriptions de la flore mauricienne tandis que les comparaisons et métaphores animalières relèvent plutôt de l'universel dans Le Voile de Draupadi.

L'étude des références temporelles des romans a permis de préciser l'époque dans laquelle sont placés les personnages, ainsi que les événements historiques auxquels il est fait allusion. Il s'est avéré que Le Sari vert remonte le plus loin dans son temps en faisant référence aux esclaves africains et malgaches, tout en ayant comme présent du récit la première moitié des années 1980. Le Voile de Draupadi, pour sa part, remonte au temps des travailleurs engagés indiens et présente des personnages qui vivent aux alentours de 1986. Rue la Poudrière, qui remonte à la période préindépendante de l'île Maurice, se déroule entre les années 1959 à 1979. C'est dire que les trois romans couvrent ensemble la période qui commence dans les années 1960 et s'achève à la fin du vingtième siècle, et mentionnent des événements plus anciens de l'histoire mauricienne. Cet ancrage temporel double permet de présenter et d'expliquer la situation de l'île Maurice d'après l'indépendance, et établit un rapprochement avec

\footnotetext{
${ }^{870}$ Bertrand Lévy, « Géographie et littérature. Une synthèse historique », Le Globe, 146, 2006, p. 16.

${ }^{871}$ Ibid., p. 41.

${ }^{872}$ Névine El Nossery, Témoignages fictionnels au féminin. Une réécriture des Blancs de la guerre civile algérienne, New York, Rodopi, 2012, p. 71.

${ }^{873}$ Ananda Devi, Le Sari vert, Paris, Gallimard, 2009. p. 131.

${ }^{874}$ Ibid. p. 188.

${ }^{875}$ Ibid. p. 210.
} 
certains événements historiques dans d'autres pays tels que la Guyane, l'Inde ou l'Afrique du Sud.

L'analyse de la dimension sociale a mis en avant le fonctionnement d'un groupe social par l'entremise d'une cellule familiale soit hindoue pour Le Voile de Draupadi et Le Sari vert, soit créole pour Rue la Poudrière, ainsi que les différentes facettes de la cohabitation des différentes ethnies et classes au sein de la société mauricienne pour les trois romans. Elle a aussi fait la lumière sur les failles d'une société où les disparités raciales et discriminatoires sont réelles et spécifiques. De plus, il est apparu que les problèmes sociaux présentés dans les trois romans, tels la pauvreté, la prostitution, l'homicide ou la violence domestique reproduisent authentiquement la situation sociale de l'île Maurice et d'ailleurs. Le 'rêve mauricien', spécifique à l'île Maurice est dénoncé dans Le Voile de Draupadi avec Dev, dont la soif pour le pouvoir et l'argent conduit au divorce et à la mort de son fils. Par contre, le 'rêve mauricien' s'est révélé bénéfique, dans Le Sari vert, pour le docteur Bissam qui s'est marié a une Hindoue de la caste supérieure et dont le métier de médecin lui a apporté argent et respect, ce qui lui a permis de grimper l'échelle sociale.

Après cet aperçu de notre recherche, nous proposons d'approfondir les questions du local et du réalisme, de l'universalisation et du réalisme, du factuel et du fictionnel qui ont formé la problématique de notre travail et qui sont sous-tendent à la contradiction que nous avons soulevé au début, à savoir celle entre le fait que l'auteure dit vouloir exprimer des réalités universelles dans ses romans et le fait que les critiques et analystes ont surtout relevé le caractère local de ses ouvrages. De plus, nous avons noté une deuxième contradiction, cette fois-ci entre ce que dit l'auteure et ce qu'elle écrit. En effet, lors de ses entretiens, Devi affirme que, si le cadre de ses romans est l'île Maurice, les sujets abordés sortent de ce cadre restreint et touchent d'autres sociétés. Mais en fait, notre analyse a démontré que même si le cadre de ces trois romans est basé sur la topographie, la flore et la faune mauriciennes, les thèmes abordés relèvent, en grande partie, de la société mauricienne. 


\section{Local et réalisme}

Lors de l'analyse de la dimension spatiale, nous avons remarqué que la ville de Port-Louis est au cœur des trois romans, même s'ils font aussi référence à d'autres villes et villages mauriciens. Si nous avons conclu que le cadre géographique des romans renvoie au panorama mauricien, il est intéressant de noter que Rue la Poudrière et Le Voile de Draupadi contiennent bien plus de références géographiques que Le Sari vert, qui est pauvre en la matière. L'auteure explique avoir été influencée par son île natale dans un premier temps :

\section{L'élan de l'écriture s'est confondu avec l'admiration exaltée que je ressentais pour la nature et dont mon besoin d'observation [...] faisait tout naturellement le centre de mon inspiration. J'avais davantage de liens, et des liens infiniment plus profonds, avec cet environnement qu'avec les gens. Les personnages que je créais naissaient de mon imaginaire, ou peut-être de rencontres brèves et fulgurantes dans une rue, sans que j'en sache rien de plus, mais les paysages décrits étaient, eux, directement empruntés à la réalité ${ }^{876}$.}

Plus tard, Devi avoue qu'elle est passée par une «période de questionnement $»^{877}$ où elle s'est « remise en cause $»^{878}$. Ceci explique que Le Sari vert délaisse la géographie mauricienne pour donner plus de place aux problèmes sociaux comme la violence domestique et la pauvreté. C'est ainsi que dans Le Sari vert, les références historiques à la période de l'esclavage, à l'épisode où de nombreux esclaves affranchis se sont jetés de la falaise du Morne afin d'échapper à la maltraitance et de retrouver la liberté, aux grèves de 1937 des petits planteurs de cannes à sucre, et aux émeutes qui ont éclaté dans l'île en janvier 1968, deux mois avant l'indépendance, entre les communautés créole et musulmane de l'île Maurice, rendent l'histoire du médecin plus réelle, vivante et crédible. En même temps, ces faits sont fictionnalisés et

\footnotetext{
${ }^{876}$ Indes réunionnaises, «L'Ecriture est le monde, elle est le chemin et le but », entretien avec Ananda Devi Indes Réunionnaises, 2003, http://www.indereunion.net/actu/ananda/intervad.htm. Web, consulté le 22 juillet 2011.

${ }^{877}$ Mar Garcia, «Entretien avec Ananda Devi », La Tortue verte: revue en ligne des littératures francophones, http://www.latortueverte.com/Entretien\%20avec\%20Ananda\%20Devi.pdf, consulté le 5 septembre 2011, p. 5.

${ }^{878}$ Ibid.
} 
interprétés pour mettre l'accent sur leur signification sociale. Pour ce roman en question, Devi affirme que l'inspiration lui est venue lorsqu'elle a entendu l'histoire d'un homme qui avait ébouillanté sa femme qui lui servait du riz mal cuit. C'est alors qu'elle a décidé de raconter la violence domestique dans la perspective de l'homme ${ }^{879}$.

Il ne fait aucun doute que Devi s'inspire d'événements et de lieux réels pour mieux ancrer ses personnages et ses histoires, tout comme elle s'inspire d'événements historiques de l'île Maurice, comme la légende orale des esclaves malgaches et africains, les conditions de vie des travailleurs engagés, les émeutes de juillet 1937 dans les établissements sucriers, le conflit racial de janvier 1968 ou la grève des dockers de 1979.

\section{Universalisation et réalisme}

Si les références spatiales, historiques et sociales des trois romans ramènent directement à un réalisme local, elles participent également d'une universalisation. Aussi, la représentation de la faune locale endémique est-elle doublée de références à diverses mythologies et de métaphores universelles. Devi touche aussi à des sujets sensibles qui débordent le monde mauricien, tels l'esclavage, la traversée et l'exil des travailleurs engagés, les conflits raciaux, les grèves des travailleurs. Par exemple, la traversée des travailleurs indiens et leurs conditions une fois dans l'île, décrites dans $L e$ Voile de Draupadi, certes réfêrent à une vérité locale mauricienne, mais aussi à une notion plus universelle de coolitude. Cette idée d'universalisation est mentionnée par Eileen Lohka :

[Il faut] que le lecteur se rend[e] compte que l'espace littéraire d'Ananda Devi [...] outrepasse ses frontières, change de forme, déstabilise le lecteur afin de mieux le stimuler à explorer d'autres possibilités, à s'ouvrir à d'autres lieux, à refuser de s'enfermer dans des conclusions trop évidentes; qu'il présente une

\footnotetext{
879 «I had heard the story of a man who poured hot water on his wife just because she served badly cooked rice made an impact on me. I wanted to narrate the issues pertaining to domestic violence from a man's perspective », Sohini Chakravorty, « Chat Author Ananda Devi talks about her latest book Le Sari vert and her love for the French Language », http://www.thehindu.com/todays-paper/tp-features/tpmetroplus/power-of-words/article1560027.ece, consulté le 6 mars 2014.
} 
topographie du décalé, de l'instable renfermant toutes les possibilités ${ }^{880}$.

Nous avons montré comme Devi se sert, dans les trois romans, du local pour dénoncer des grands maux sociaux tels que la prostitution, l'alcoolisme, la violence conjugale et la croyance aveugle dans la religion et les traditions. Norbert Louis estime d'ailleurs que l'œuvre de Devi « répond à une demande de l'imaginaire mauricien mais [...] peut s'interpréter en fonction de divers imaginaires ${ }^{881}$. De même, Eileen Lohka pense que les romans de Devi sont «bien ancrés dans son terroir mauricien mais [...] que l'écriture transcende les frontières - spatiales, temporelles [...] pour offrir au lecteur une manière toujours nouvelle d'appréhender le monde ${ }^{882}$.

En plus, Ananda Devi universalise la question de la prostitution et de la pauvreté qui sont des problèmes sociaux qui ne touchent pas exclusivement l'île Maurice. Mallacre, Tapsy, Edouard, Marie, Paule, victimes de leur milieu social, représente chacun le cas typique du chômeur alcoolique brisé par les circonstances, du proxénète et de la prostituée, de l'épouse, personnage qui veut sortir de sa condition sociale par tous les moyens possibles alors que Tapsy est victime de sa naïveté. Basée sur l'oppression ou la violence les relations des couples sont vouées à l'échec.

Nous avons aussi montré comment Devi est parvenue à inscrire l'universel, comme elle l'espérait, dans Rue la Poudrière, Le Voile de Draupadi et Le sari vert:

Mes romans parlent d'individus qui débouchent sur des archétypes universels [...]. Aucun de mes romans ne vise à être une analyse anthropologique de la société mauricienne ${ }^{883}$.

A propos de cette question du local et de l'ailleurs, Lohka écrit que l'espace de Devi, «fluide [...], multiple et polymorphe, perçu autant de façon abstraite que concrète, se défait de la notion de frontières et englobe de ce fait une vision plus large

\footnotetext{
${ }^{880}$ Eileen Lohka, «Outrepasser le lieu et ouvrir un espace de création. Le cas d'Ananda Devi », Nouvelles études francophones, 28, 2013, p. 31.

${ }^{881}$ Norbert Louis, « Ananda Devi : le pari de l'universel à partir du local », http://www.genderlinks.org.za/article/ananda-devi--le-pari-de-luniversel-partir-du-local-2010-02-25-1, consulté le 15 juillet 2011.

${ }^{882}$ Eileen Lohka, «Outrepasser le lieu et ouvrir un espace de création. Le cas d'Ananda Devi », Nouvelles études francophones, 28, 2013, p. 36.

${ }^{883}$ Nathalie Philippe, «Explorer l'île pour atteindre l'universel. Retour sur l'œuvre d'Ananda Devi », Les Dépêches de Brazzaville, http://www.brazzaville-adiac.com/medias/dossiertele/suplit7.pdf, consulté le 13 mars 2013.
} 
de ce que l'écrivain tente de mettre en mots ${ }^{884}$. Cette idée est d'ailleurs confirmée par l'auteure elle-même lors d'un de ses entretiens :

L'île Maurice de mes fictions est avant tout une île Maurice mentale, rêvée, et s'il est vrai que les jeunes [...] habitent à Port-Louis, [ils] pourraient très bien, à quelques changements près, habiter ailleurs ${ }^{885}$.

\section{Le factuel et le fictionnel : le « mensonge du romancier »}

Devi tisse si bien la réalité mauricienne dans ses histoires fictives qu'il devient très difficile, à première lecture, de différencier le fictionnel du factuel. Vu qu'il n'a pas été facile de tirer la ligne de démarcation entre la fiction et la réalité dans les trois romans de Devi, nous trouvons utile d'emprunter le terme d'El Nossery pour conclure que les trois romans sont des «témoignage[s] fictionnel[s] ${ }^{886}$ où l'incorporation du réel aide à situer le récit

Entre le factuel et le fictionnel, l'éthique et l'esthétique, supprimant les frontières poreuses entre les genres et prouvant finalement que la fiction s'avère dans certains cas plus véridique qu'un manuel historique étant donné que le recours à l'événementiel et au témoignage participe à la littérarité du texte grâce à la transformation et l'esthétisation qu'il subit ${ }^{887}$.

Nos analyses ont bien montré comment Devi fait ressortir la ségrégation raciale, l'importance de la caste, de la couleur de la peau, et du rêve mauricien comme réponse aux inégalités sociales qui caractérisent l'île Maurice ${ }^{888}$, en incorporant fiction et réel

\footnotetext{
${ }^{884}$ Eileen Lohka, «Outrepasser le lieu et ouvrir un espace de création. Le cas d'Ananda Devi », Nouvelles études francophones, 28, 2013, p. 28- 29.

${ }_{885}$ Mar Garcia, «Entretien avec Ananda Devi », La Tortue verte: revue en ligne des littératures francophones, http://www.latortueverte.com/Entretien\%20avec\%20Ananda\%20Devi.pdf, consulté le 5 septembre 2011, p. 3.

${ }^{886}$ Névine El Nossery, Témoignages fictionnels au féminin. Une réécriture des Blancs de la guerre civile algérienne, New York, Rodopi, 2012, p. 220.

${ }_{887}^{8}$ Ibid.

888 «Ce qui me marque, à Maurice, c'est ce besoin de consommation toujours plus flagrant, cette nécessité d'acquérir tous les apparats de la société matérielle et, du coup, cet écart qui se creuse entre les plus pauvres et les plus nantis, car la vie est très chère », Fabien Mollon, «Ananda Devi : il y a une violence latente à Maurice», http://www.jeuneafrique.com/Article/JA2726p082-083.xml0/, consulté le 22 mai 2013.
} 
pour tirer la sonnette d'alarme sur plusieurs réalités que les Mauriciens ne veulent pas voir. Elle reconnait volontiers ses intentions :

Je suis une sorte de représentante de mon pays mais j'ai parfois le sentiment que les gens ont peur d'aller jusqu'au bout de ce que j'énonce. Je dis beaucoup de choses sur la société de l'île Maurice ${ }^{889}$. Ce que je dénonce ? C'est l'éternelle emprise du « communalisme », c'est-à-dire des divisions d'ordre ethnique, dans cette société, avec son cortège de préjugés, de mépris, d'incompréhension, ou plutôt de refus de comprendre. C'est le fait que, de plus en plus, elle se transforme en une société régie par l'argent et par le culte de la réussite matérielle. C'est que de nombreux parents démissionnent par rapport à l'éducation véritable et aux principes qu'ils sont censés inculquer à leurs enfants [...] C'est que c'est une pseudomoralité basée sur des croyances religieuses aveugles et sans questionnement qui prend le dessus [...] on ne fait que renforcer les clivages en créant des centres culturels à foison qui chemineront en parallèle sans jamais se rencontrer ni se comprendre ${ }^{890}$.

En particulier, nous avons établi que dans les trois romans étudiés, Devi se concentre sur les communautés hindoue, musulmane et créole pour dénoncer les inégalités sociales. Ce dont elle parle dans un de ses entretiens accordé à Fabien Mollon :

$$
\begin{aligned}
& \text { Ce qu'on appelle à Maurice des communautés, qui } \\
& \text { peuvent être religieuses ou de race, entre hindous et } \\
& \text { musulmans, entre créoles et personnes d'origine } \\
& \text { indienne ... ou alors à l'intérieur même de la }
\end{aligned}
$$

\footnotetext{
${ }^{889}$ Muriel Steinmetz, « Ananda Devi. Tempête sous un très vieux crâne à l'île Maurice », 2009, L'Humanité-Culture, http://www.lettresfrontiere.net/dms-static/c0586918-5575-4f26-9c6dceb199242e33/Ananda\%20Devi.pdf, consulté le 13 mars 2012.

${ }^{890}$ Indes réunionnaises, «L'Ecriture est le monde, elle est le chemin et le but », entretien avec Ananda Devi Indes réunionnaises, 2003, http://www.indereunion.net/actu/ananda/intervad.htm. Web, consulté le 22 juillet 2011.
} 
communauté indienne, par exemple, il y a des clivages de caste ou de langue ${ }^{891}$.

Eileen Lohka avait d'ailleurs noté que Devi choisit de donner la voix à ceux qui sont mis de côté, aux classes défavorisées et aux femmes violentées ${ }^{892}$.

En outre, notre recherche a dévoilé que l'ancrage temporel de certains faits historiques est peu fiable ou vague. Ainsi, dans Rue la Poudrière, l'âge de Paule lorsqu'elle part habiter aux Dockers' Flats reste indéterminable. Dans Le Voile de Draupadi le présent du récit n'est jamais complètement certain et dans Le Sari vert, les événements de juillet 1937 sont amalgamés à ceux de janvier 1968 par l'anachronisme d'environ quarante-huit ans. Dans une communication personnelle, l'auteure avoue avoir pris des libertés en ce qui concerne le temps dans Le Sari vert ${ }^{893}$. Cet anachronisme, qui constitue un amalgame des émeutes en 1968 et des grèves des petits planteurs de 1937, a pour effet d'accentuer l'importance qu'ont eue les idées d'émancipation dans l'histoire de la société mauricienne.

Il ressort de tout cela que, malgré le réalisme de ses univers, Ananda Devi "fictionnalise" ses écrits en rendant plus universels certains faits historiques mauriciens et certaines situations sociales locales ; comme elle le dit elle-même encore dans un de ses entretiens :

Maurice [est] une source inépuisable d'inspiration. En dépit de ses apparences d'exiguïté, cette terre contient une infinité de mondes. Malgré des mécanismes d'interaction et d'intégration encore défaillants ou artificiels, les différents groupes ethniques recomposent au quotidien la singularité de leur univers. Ce microcosme imprévisible me permet dans chacune de mes histoires d'entrer dans un monde différent ${ }^{894}$.

\footnotetext{
${ }^{891}$ Fabien Mollon, « Ananda Devi : il y a une violence latente à Maurice », http://www.jeuneafrique.com/Article/JA2726p082-083.xml0/, consulté le 22 mai 2013. ${ }^{892}$ Eileen Lohka, lors d'une communication personnelle du 11 juin 2013 faite à Grand-Baie, île maurice. 893 « With regard to Sari vert, I have taken considerable liberties with the time frame [...] the riots I was referring to were in 1943, but this, and the "torn underwear scene" during the independence riots (1968) really do not fit in with the age of the narrator. But I tried to leave the year during which the story is actually taking place fairly vague, because of this. My fiction does tend to play with historical facts a bit », communication personnelle datant du 09 août 2012.

${ }^{894}$ Marie Abraham, «L'Ile Maurice : source inépuisable d'inspiration », http://www.lehman.cuny.edu/ile.en.ile/paroles/devi_maurice.html, consulté le 18 mars 2013.
} 
C'est exactement ce que Lohka avait remarqué en affirmant qu'en « outrepassant les frontières entre le fictionnel et le réel [...] Ananda Devi (r)établit l'espace de l'écriture comme lieu de renouvellement ${ }^{895}$.

En tant qu'anthropologue ${ }^{896}$, Devi met en avant, avec réalisme, le passé de l'île Maurice. D'ailleurs, elle reconnaît lors d'un entretien accordé à Muriel Steinmetz: «mes études en anthropologie sociale ont bien sûr aiguisé mon regard sur la société ${ }^{897}$. Comme romancière, Devi "fictionnalise" et "universalise" cette réalité mauricienne ${ }^{898}$. Un de ses protagonistes, Subhadra, dans son roman Indian Tango, parle du "mensonge du romancier" :

L'objectif du romancier est de se cacher le mieux possible derrière ses mots. Mais si d'aventure l'envie lui prend de se révéler, il le fera de telle façon que personne ne reconnaîtra la vérité. Méfiez-vous du mensonge du romancier $^{899}$.

Devi elle-même avoue qu'écrire c'est mentir : « D'accord. J'écris, donc je mens ${ }^{900}$.

En fait, dans ses trois romans, l'auteure jongle entre le factuel et la fiction, brouillant ainsi les frontières entre ces deux entités en tissant dates et faits réels dans sa fiction de manière presqu'indissociable. Elle arrive ainsi, comme tous les romanciers, à créer un «troisième espace » qui reconnaît que la distinction entre fait et fiction est trompeuse, que la fiction est un espace où se mêlent réalité et imaginaire, réel et inventé. En fait, la romancière a recours à certaines réalités toponymiques et topographiques, historiques et sociales pour rendre vraisemblable son histoire fictionnelle. Si l'île Maurice forme le cadre des trois romans de Devi comme l'ont noté

${ }^{895}$ Eileen Lohka, «Outrepasser le lieu et ouvrir un espace de création. Le cas d'Ananda Devi », Nouvelles études francophones, 28, 2013, p. 34.

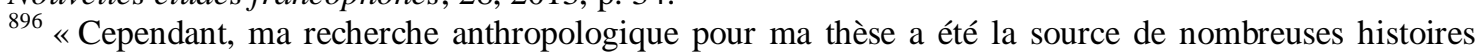
que j'ai utilisées par la suite dans mes livres », Mar Garcia, «Entretien avec Ananda Devi », La Tortue verte: revue en ligne des littératures francophones,

http://www.latortueverte.com/Entretien\%20avec\%20Ananda\%20Devi.pdf,

3 consulté le 5 septembre 2011, p. 1.

${ }^{897}$ Muriel Steinmetz, «Ananda Devi. Tempête sous un très vieux crâne à l'île Maurice », 2009, L'Humanité-Culture, http://www.lettresfrontiere.net/dms-static/c0586918-5575-4f26-9c6d-

ceb199242e33/Ananda\%20Devi.pdf, consulté le 13 mars 2012.

${ }^{898}$ Nathalie Philippe, « Explorer l'île pour atteindre l'universel. Retour sur l'œuvre d'Ananda Devi », Les Dépêches de Brazzaville, http://www.brazzaville-adiac.com/medias/dossiertele/suplit7.pdf, consulté le 13 mars 2013.

${ }^{899}$ Ananda Devi, Indian Tango, Paris, Gallimard, 2007, p. 53.

900 Ananda Devi, Les Hommes qui me parlent, Paris, L'Harmattan, 2011, p. 184. 
les analystes et critiques à propos de son écriture et comme l'admet l'auteure, ils présentent aussi une dimension sociologique mauricienne importante. Manifestement, les trois romans étudiés placent les personnages à l'île Maurice et abordent avec un certain réalisme les différentes facettes de la société mauricienne, même si les problèmes sociaux ne sont pas toujours uniquement mauriciens puisque nous avons observé que les romans ont également une dimension, surtout sociologique, plus universelle. Cette focalisation multiple, à la fois locale et universelle provient du fait que Devi mêle le point de vue endogène et le point de vue exogène ${ }^{901}$ en entrelaçant la vision de l'autochtone familier du monde décrit avec la vision de l'étranger qui observe son environnement avec un regard nouveau et plus détaché. De la sorte, Devi parvient à juxtaposer avec réalisme, le local et l'universel dans ses romans.

${ }^{901}$ Bertrand Westphal, La Géocritique. Réel, fiction, espace, Paris, Les Editions de Minuit, 2007, p. 208. 


\section{Bibliographie}




\section{Romans d'Ananda Devi étudiés}

Devi, Ananda, Rue la Poudrière, Abidjan, Nouvelles Editions Africaines, 1988.

Devi, Ananda, Le Voile de Draupadi, Paris, L'Harmattan, 1993.

Devi, Ananda, Le Sari vert, Paris, Gallimard, 2009.

\section{Entrevues d'Ananda Devi consultées}

Abraham Marie, «L'Ile Maurice : source inépuisable d'inspiration », http://www.lehman.cuny.edu/ile.en.ile/paroles/devi_maurice.html, consulté le 18 mars 2013.

Assouline Stillman, Dinah, «A Quiet Author's Written Rebellion. An Interview with Ananda Devi », World Literature Today, 87, 2013, p. 23-27.

Borges, Andre, « Jaipur Literature Festival 2014: Music of Languages. An Interview with Ananda Devi », http://zeenews.india.com/entertainment/bookworm/jaipurliterature-festival-2014/music-of-languages-an-interview-with-anandadevi_3165.htm, consulté le 13 juin 2014.

Chakravorty, Sohini, « Chat Author Ananda Devi talks about her latest book Le Sari Vert and her Love for the French Language », http://www.thehindu.com/todayspaper/tp-features/tp-metroplus/power-of-words/article1560027.ece, consulté le 6 mars 2014

Chanda, Tirthankar, « Ananda Devi : " "Le père ne s'excuse jamais du mal qu'il a perpétré autour de lui" », 2009, Revue de presse RFI, http://www.lettresfrontiere.net/dms-static/c0586918-5575-4f26-9c6dceb199242e33/Ananda\%20Devi.pdf, consulté le 3 septembre 2011.

Earally, Abdoolah, « Ananda Devi : “Il y aurait une autre manière de célébrer l'indépendance" », http://www.montraykreyol.org/spip.php?article5629, consulté le 29 octobre 2013.

Garcia, Mar, «Entretien avec Ananda Devi », La Tortue verte : revue en ligne des littératures francophones, http://www.latortueverte.com/Entretien\%20avec\%20Ananda\%20Devi.pdf, consulté le 5 septembre 2011.

Hawkins, Peter, «An Interview with Ananda Devi », Wasafiri, 26, 2011, p. 8-13.

Heminway, Annie, « Ananda Devi sur Les Hommes qui me parlent », http://www.lindaleith.com/posts/view/172, consulté le 20 mai 2013.

Indes réunionnaises, «L'Ecriture est le monde, elle est le chemin et le but », entretien avec Ananda Devi, Indes réunionnaises, 2003, http://www.indereunion.net/actu/ananda/intervad.htm. Web, consulté le 22 juillet 2011.

Issur, Kumari, « Un univers d'émotions et de sensations : l'écriture intimiste d'Ananda Devi. Entretien de Kumari Issur avec l'auteure », Paroles gelées, 27, 2012, p. 71-82. 
Léchot, Bernard, «Ananda Devi : une voix de l'océan Indien », http://www.swissinfo.ch/fre/Dossiers/LArchipel francophone/Le francais selo n.../Ananda_Devi:_une_voix_de_lOcean_indien.html?cid=8828986, consulté le 27 octobre 2011.

Le Mauricien, « Ananda Devi, écrivain : j'essaie de montrer l'alchimie entre le vécu, l'imaginaire et la littérature $»$, http://www.lemauricien.com/article/ananda-devi\% $3 \%$ A9crivain $\% \mathrm{C} 2 \% \mathrm{~A} 0-\% \mathrm{C} 2 \% \mathrm{AB} \% \mathrm{C} 2 \% \mathrm{~A} 0 \mathrm{j} \% \mathrm{E} 2 \% 80 \% 99$ essaie-montrer1\%E2\%80\%99alchimie-entre-v\%C3\%A9cu-1\%E2\%80\%99imaginaire-et-lalitt\%C3\%A9ratur, consulté le 27 octobre 2011.

Le Mauricien, «Ananda Devi : l'écriture et la vie profonde », http://www.lemauricien.com/article/ananda-devi-1\%C3\%A9criture-et-la-vieprofonde, consulté le 27 octobre 2011.

Le Mauricien, «Entretien avec Ananda Devi : «Il y a une scission de moi-même ; dans mes livres j'ose tout », http://www.lemauricien.com/article/ananda-deviecrivain $\% \mathrm{C} 2 \% \mathrm{~A} 0-\% \mathrm{C} 2 \% \mathrm{AB} \% \mathrm{C} 2 \% \mathrm{~A} 0 \mathrm{il}-\mathrm{y}$-scission-moi-meme\%C2\%A0-meslivres-j\%E2\%80\%99ose-tout\%C2\%A0\%C2\%BB, consulté le 21 décembre 2012.

Louis, Norbert, « Ananda Devi : le pari de l'universel à partir du local », http://www.genderlinks.org.za/article/ananda-devi--le-pari-de-luniversel-partirdu-local-2010-02-25-1, consulté le 15 juillet 2011.

Mollon, Fabien, «Ananda Devi : il y a une violence latente à Maurice », http://www.jeuneafrique.com/Article/JA2726p082-083.xml0/, consulté le 22 mai 2013.

Nkonlak, Romuald, Jules, « Ananda Devi, Angeline Solange Bonono : l'expression "littérature féminine" ne veut rien dire », allAfrica, Quotidienmutations, http://fr.allafrica.com/stories/200701050490.html, consulté le 15 juillet 2011.

Philippe, Nathalie, «Explorer l'île pour atteindre l'universel. Retour sur l'œuvre d'Ananda Devi », Les Dépêches de Brazzaville, http://www.brazzavilleadiac.com/medias/dossiertele/suplit7.pdf, consulté le 13 mars 2013.

Spear, Thomas, «Ananda Devi in conversation with Thomas C. Spear. From "Literary Talks Series" of the FIAF », http://www.youtube.com/watch?v=nyo66ZUuFAU, consulté le 24 mai 2013.

Steinmetz, Muriel, « Ananda Devi. Tempête sous un très vieux crâne à l'île Maurice », 2009, L'Humanité-Culture, http://www.lettresfrontiere.net/dms-static/c05869185575-4f26-9c6d-ceb199242e33/Ananda\%20Devi.pdf, consulté le 13 mars 2012.

Sultan, Patrick, « Ruptures et héritages. Entretien avec Ananda Devi », 2001, http://orees.concordia.ca/numero3/essai/sultan.shtml, consulté le 8 mai 2011.

Torabully, Khal, «Entretien avec Ananda Devi », Notre librairie, 142, 2000, p. 58-65.

Waters, Julia, « Ananda Devi as Writer and Translator: an interview with Julia Waters », Intimate Enemies. Translation in Francophone Contexts, Francophone Postcolonial Studies, 4, 2013, p. 117-123. 


\section{Critiques, articles, ouvrages et sites relatifs à Ananda Devi}

Abada Medjo, Jean Claude, «Poétique de la ville dans l'œuvre d'Ananda Devi », http://revel.unice.fr/loxias/index.html?id=7044, consulté le 17 janvier 2013, p. $1-15$.

Abada Medjo, Jean-Claude, «Utopie identitaire et traversée des genres dans l'œuvre d'Ananda Devi », Les Cahiers du GRELCEF, Les écrits contemporains des femmes de l'océan Indien et des Caraïbes, 3, 2012, p. 139-156.

Anderson, Jean, «Can the Subjected Speak? Giving a Voice to the Voiceless in Moi, l'interdite by Ananda Devi and Une femme de rien by Maryline Desbiolles », Women in French Studies, 2008, p. 108-115.

Anderson, Jean, « The Other Side of the Postcard: Rewriting the Exotic Beach in Works by Titaua Peu, Chantal Spitz (Tahiti) and Nathacha Appanah (Mauritius) », Dalhousie French Studies, 94, Spring 2011, p. 5-12.

Anonyme, «Ananda Devi lauréate 2010 du "Prix Louis-Guilloux" », http://www.lexpress.mu/article/ananda-devi-laur\%C3\%A9ate-2010-du$\% \mathrm{C} 2 \% \mathrm{AB}-$ prix-louis-guilloux-\%C2\%BB, consulté le 20 octobre 2011.

Bannerjee, Rohini, « Le Sari vert », Journal du conseil international d'études francophones (CIEF), 25, 1, 2010.

Bannerjee, Rohini, « The Kala Pani Connection: Francophone Migration Narratives in the Caribbean Writing of Raphaël Confiant and the Mauritian Writing of Ananda Devi », Anthurium: a Caribbean Studies Journal, 7, 2009, p. 1-11.

Bannerjee, Rohini, « L'Espace littéraire mauricien : l'hétérolinguisme dans l'œuvre francophone d'Ananda Devi », Contemporary French and Francophone Studies, 15, 2011, p. 505-512.

Bannerjee, Rohini, « Daughter Forsaken: la résistance of the Indo-Mauritian Girl Child in Ananda Devi's Novel » dans India in the World, Cristina M. GámezFernández, Antonia Navarro-Tejero, Newcastle, Cambridge Scholars Press, 2011, p. 123-134.

Beesoondial, Ashish, « The Voice of the Other: the Female Gothic and Ananda Devi's Moi l'Interdite », Journal of Mauritian Studies, 2, 2003, p. 105-129.

Bénac-Giroux, Karine, « Le Martyre des femmes dans Le Sari vert d'Ananda Devi », dans Libertés et oppressions, Paris, L'Harmattan, p. 103-113.

Bragard, Véronique, «Cris des femmes maudites, brûlures du silence, la symbolique des éléments fondamentaux dans l'œuvre d'Ananda Devi », Notre Librairie, 142, 2000, p. 66-73.

Bragard, Véronique, « Ananda Devi », http://www.lehman.cuny.edu/ile.en.ile/paroles/devi.html, consulté le 2 mai 2012.

Bragard, Véronique, «Eaux obscures du souvenir. Femme et mémoire dans l'œuvre d'Ananda Devi », Convergences and Interferences: Newness in Intercultural Practices. Ecrire d'une nouvelle ere/aire, Amsterdam et New York, Rodopi, 8, 2001, p. 187-199. 
Bragard, Véronique, « Regards croisés sur la mémoire coolie des Antilles aux Mascareignes », Nouvelles études francophones, 21, 2006, p. 163-180.

Burri, Julien, « Ananda Devi : la rage et le sucre », http://www.hebdo.ch/ananda_devi_la_rage_et_le_sucre_135983_html, consulté le 7 août 2013.

Cazenave, Odile, «Les hommes qui me parlent d'Ananda Devi. Un nouvel espace pour se dire ? », Nouvelles études francophones, 28, 2013, p. 39-52.

Chavy Cooper, Danielle, «Rue la Poudrière », World Literature Today, 68, 1990, p. 515.

Chavy Cooper, Danielle, «Le Voile de Draupadi », World Literature Today, 68, 1994, p. 640-641.

Chemla, Yves, « Ananda Devi : Le Sari vert», http://www.cecong.org/index.php?option=com_content $\&$ view=article \&id=416:ananda-devi-lesari-vert-205-\&catid=131:chroniques-de-livres\&Itemid=224, consulté le 11 avril 2013.

Chitour Mangin, Marie-Françoise, « Appropriation linguistique et stratégies d'écriture chez deux romancières de l'océan Indien (Monique Agénor et Ananda Devi) », Appropriation de la langue dans les littératures francophones de l'Afrique subsaharienne, du Maghreb et de l'océan Indien, actes des journées scientifiques des réseaux de chercheurs concernant la langue et la littérature, Angers, université d'Angers, 2006, p. 83-90.

Cunniah, Bruno, «La Textualisation de la dissidence chez Ananda Devi et Shakuntala Boolel », Les Cahiers du GRELCEF, 3, 2012, p. 123-137.

Damlé, Amaleena, «Phantasmal Relics, Psychoanalytical and Deconstructive Ghosts in Moi, l'interdite and Pagli, Amnesia and Haunting », Amnesia: Private and Public Memory in Modern French Culture, Peter Collier, Anna Magdalena Elsner, Olga Smith, eds, Bern, Peter Lang, 2009, p. 229-240.

Delmeule, Jean-Christophe, « Les hybridations mensongères, (Ananda Devi, Jean-Luc Raharimanana, Abdourahman Waberi) », La Tortue verte : revue en ligne des littératures francophones, http://www.latortueverte.com/Article2\%20hybridations\%20mensongeres\%20JC \%20Delmeule.pdf, consulté le 28 juillet 2011.

De Tilly, Marine, «"Le Sari vert” d'Ananda Devi », http://www.lepoint.fr/livre/le-sarivert-d-ananda-devi-08-02-2011-1293168 79.php, consulté le 19 août 2013.

Devi, Ananda, Les hommes qui me parlent, Paris, L'Harmattan, 2011.

Devi, Ananda, Indian Tango, Paris, Gallimard, 2007.

Effertz, Julia, «Le prédateur, c'est moi : l'écriture de la terre et la violence féminine dans l'œuvre d'Ananda Devi », Violence in French and Francophone Literature and Film, James Day, Amsterdam, Rodopi, 2008, p. 71-82.

Fonkoué, Ramon, «Voix de femmes et figures de mâle en littérature francophone : Nicole Brossard et Maryse Condé », Nouvelles études francophones, 25, 2010, p. 75-89.

Galibert, Nivoelisoa, «Port de badge et vision panoptique : les Dockers' Flats de PortLouis dans Rue la Poudrière (1988) d'Ananda Devi », Revue historique des Mascareignes, 5, 2004, p. 95-109. 
Ganapathy-Doré, Geeta, «Babyji d'Abha Dawesar et Indian Tango d'Ananda Devi : une étude comparée de la femme et de l'Inde contemporaine », Images changeantes de l'Inde et de l'Afrique, Paris, Harmattan, 2011, p. 179-193.

Gannier, Odile, « Jeunes déboussolés dans le Pacifique, dans les romans d'Alan Duff, Sia Figiel, Chantal T. Spitz, Albert Wendt et Ananda Devi », http://revel.unice.fr/loxias/index.html?id=2937, consulté le 5 mars 2013.

Garcia, Mar, «La Colonie pénitentiaire : exotisme et altérité dans Soupir d'Ananda Devi », dans India and the Diasporic Imagination/L'Inde et l'imagination diasporique, 37, 2006, p. 443-459.

Githire, Njeri, « The Semiotics of (Not) Eating: Fasting, Anorexia, and Hunger Strike in Ananda Devi's Le Voile de Draupadi », Nottingham French Studies, 48, 1, 2009, p. 82-93.

Golay, Annabelle, «Féminisme et postcolonialisme : Beauvoir, Fanon et la guerre d'Algérie », International Journal of Francophone Studies, 10, 2007, p. 407424.

Intertitres. L'Ecriture de fil en aiguille, « Ananda Devi : prix Louis Guilloux 2010 », http://cibamike.wordpress.com/2010/08/, consulté le 17 janvier 2013.

Itsieki Putu Basey, Jean de Dieu, « Amour, folie, liberté : la dissidence féminine dans l'écriture d'Ananda Devi », La Tortue verte : revue en ligne des littératures francophones, http://w3.gril.univ-tlse2.fr/analyses/A2012/ITSIEKI.pdf, consulté le 25 janvier 2013.

Jean-François, Emmanuel Bruno, «L'Ecriture de la déportation chez les écrivaines mauriciennes contemporaines : entre mémoire de la violence et violence de la mémoire », Les Cahiers du GRELCEF, Les écrits contemporains des femmes de l'océan Indien et des Caraïbes, 3, 2012, p. 103-121.

Kistnareddy, Ashwiny, «Interrogating Identity: psychological Dislocations in Ananda Devi's Novels », Dalhousie French Studies, 94, Spring 2011, p. 27-38.

Kistnareddy, Ashwiny, «Entre nomadisme et sédentarité : l'identité îlienne dans Pagli d'Ananda Devi », Le Panoptique : Perspectives sur les enjeux contemporains, 2008, http://www.lepanoptique.com/sections/arts-litterature/entre-nomadismeet-sedentarite-lidentite-ilienne-dans-pagli-dananda-devi/, consulté le 2 mai 2012.

Le Mauricien, «Littérature : le premier prix Mokanda décerné à Ananda Devi », http://www.lemauricien.com/article/litterature-premier-prix-mokanda-decerneananda-devi, consulté le 2 mai 2012.

Le Matinal News Service, « Le Sari Vert d'Ananda Devi : une constance littéraire qui force l'admiration », http://www.lematinal.com/mobile/magazine/268-sari-vertdAnanda-Devi-une-constance-littraire-qui-force-ladmiration.html, consulté le 2 mai 2012.

L'Express. Culture, « Ananda Devi lauréate 2010 du prix Louis-Guilloux », http://www.lexpress.mu/article/ananda-devi-laur\%C3\%A9ate-2010-du\%C2\%AB-prix-louis-guilloux-\%C2\%BB, consulté le 20 octobre 2011.

Lionnet, Françoise, «Evading the Subject: Narration and the City in Ananda Devi's Rue la Poudrière », L'Esprit créateur. Post-Colonial Women's Writing, 33, 1993, p.9-22. 
Lionnet, Françoise, " "New world" Exiles and Ironists from Evariste Parny to Ananda Devi », Postcolonial Poetics. Genre and Form, 2, 2011, p. 13-34.

Lohka, Eileen, « De la terre à la terre, du berceau à la tombe, l'île d'Ananda Devi », Nouvelles études francophones, 23, 1, 2008, p. 154-162.

Lohka, Eileen, « Outrepasser le lieu et ouvrir un espace de création. Le cas d'Ananda Devi », Nouvelles études francophones, 28, 2013, p. 27-38.

Magdelaine-Andrianjafitrimo, Valérie, « De Sita à Draupadi. Les ambivalences d'Anjali et de Vasanti dans Le Voile de Draupadi, d'Ananda Devi », La Revue "Vues d'ailleurs », 6, 2011-2012, p. 1-25.

Marson, Magali, «Carnalité et métamorphoses chez Ananda Devi », Indispensables Animaux, Notre librairie, 163, 2006, p. 71-76.

Marson, Magali, «Ananda Devi. Le Sari vert», Cultures Sud, la revue en ligne des littératures $d u$ sud, http://www.culturessud.com/contenu.php?id=42, consulté le 2 mai 2012.

Martin, Celina, «Ananda Devi et T.S. Eliot : réécriture, palimpseste et poétique de la différence », Cahiers suisses de littérature comparée, 37, 2006, p. 249-259.

Meitinger, Serge, «L'Innocence meurtrière : humanité et animalité dans les récits d'Ananda Devi », Francofonia, 17, 2008, p. 161-178.

Meitinger, Serge, «Le Vol librement choisi de l'oiseau. À propos des nouvelles d'Ananda Devi », Littérature critique francophone de l'Afrique subsaharienne et de l'océan Indien, 2007, p. 1-16.

Meitinger, Serge, «Avatars de la déesse-indianité, féminité et universalité du féminin dans l'œuvre d'Ananda Devi », La Revue de ressources.org, 2010 (2008), http://www.larevuedesressources.org/avatars-de-la-deesse,921.html, consulté le 1 août 2011.

Meitinger, Serge, «Les Fantastiques dans les nouvelles d'Ananda Devi », Iris : les cahiers du Gerf, 26, 2004, p. 17-28.

Mihalovici, Florina-Liliana, «La "Guerre silencieuse" de la femme dans une société patriarcale qui la dévore : Le Sari vert d'Ananda Devi », dans Parole au silence, Limoges, Pulim, 2012, p. 103-113.

Mouralis, Bernard, «La Littérature africaine et le fait colonial : quels traitements aujourd'hui ?», Cultures Sud: Notre librairie, Revue des littératures d'Afrique, des Caraïbes et de l'océan Indien, 165, 2007, p. 91-98.

Poché, Jean-Marc, «Le prix des Cinq Continents de la Francophonie à une Mauricienne », http://www.radiomoris.com/forum/nouvelles-de-lilemaurice/4382-le-prix-des-cinq-continents-de-la-francophonie-unemauricienne.html, consulté le 25 juillet 2011.

Raharimanana, Jean-Luc, «Moi l'interdite », Notre librairie, 142, 2000, p. 74-75.

Ramharai, Vicram, « Ananda Devi : repenser l'identité de la femme mauricienne », Notre librairie, 146, 2001, p. 110-112.

Ramharai, Vicram, «La Ville de Port-Louis dans Rue la Poudrière d'Ananda Devi », L'océan Indien dans les littératures francophones, Paris, Karthala, 2001, p. 706. 
Ramharai, Vicram, «Le Champ littéraire mauricien », Revue de littérature comparée, 318, 2006, p. 173-194.

Ramharai, Vicram, «Voyage, espace et postcolonialisme dans The Snake Spirit de Chaya Parmessur », Journal of Mauritian Studies. Voyages, Space and Encounters. South West Region of the Indian Ocean, 1, 2009, p. 101-129.

Ramharai, Vicram, «La Littérature mauricienne d'expression anglaise : problèmes et perspectives », Journal of Mauritian Studies, 2, 1998, p. 1-39.

Ramharai, Vicram, «La Litérature des années soixante à Maurice : reflet ou refus d'une société en mutation », http://www.cresoi.fr/IMG/pdf/revue_mascareigne_04part8.pdf, consulté le 11 juillet 2014.

Ramharai, Vicram, «Récits postcoloniaux, retour colonial et diaspora indienne à Maurice », http://revel.unice.fr/symposia/actel/?id=417, consulté le 11 juillet 2014.

Ravi, Srilata, «Religion, Health and Hindu Woman in Mauritius: Ananda Devi's Le Voile de Draupadi », Kunapipi, Journal of Postcolonial Writing, 28, 2006, p. 67-78.

Rivière, Marc, Serge, «The Impact of Exile on the Construction of a Cross-Cultural Identity. Two Case-Studies: Matthew Flinders and Daisy White », Journal of Mauritian Studies, 4, 2009, p. 26-43.

Rousseau, Christine, «Le Sari vert d'Ananda Devi : un terrible monologue », http://www.lemonde.fr/livres/article/2009/11/12/le-sari-vert-d-anandadevi_1266068_3260.html, consulté le 12 avril 2013.

Samboo, Sachita, «Vers une écriture de l'interculturel : déconstruction et démystification des mythes hindous chez Ananda Devi », La Diaspora indienne dans l'histoire des îles et pays de l'océan Indien, actes du colloque international organisé du 20 au 22 janvier 2010, La Réunion, 2010, p. 289- 299.

Sultan, Patrick, «Littérature : Ananda Devi », http://www.lehman.cuny.edu/ile.en.ile/paroles/devi.html, consulté le 20 mai 2013.

Tyagi, Ritu, «"Feminine” Desire in Ananda Devi’s Narratives », Dalhousie French Studies, 94, 2011, p. 65-75.

Tyagi, Ritu, Ananda Devi. Feminism, Narration and Polyphony, New York, Rodopi, 2013.

Tyagi, Ritu, « Rethinking Identity and Belonging. "Mauritianness" in the Work of Ananda Devi », dans Islanded Identities.Constructions of Postcolonial Cultural Insularity, New York, Rodopi, 2011, p. 89-108.

Union des femmes de la Martinique, « Journée internationale pour l'élimination des violences envers les femmes », http://www.unionfemmesmartinique.com/?article-683-textes-et-poemes-pourla-journee-du-25-nov-a-fort-de-france, consulté le 5 décembre 2012.

Uzunhasan, Inci, «L'Espace-Temps et la quête identitaire chez Ananda Devi », http://s343802320.onlinehome.fr/ villaGillet/?p=455, consulté le 17 janvier 2013.

Waters, Julia, " "Ton continent est noir": Rethinking Feminist Metaphors in Ananda Devi's Pagli », Dalhousie French Studies, 68, 2004, p. 45-55. 
Waters, Julia, «From Continents noirs to Collection blanche: From Other to Same? The Case of Ananda Devi », Postcolonial Literatures of the Francophone Indian Ocean, 2, 2008, p. 55-73.

Waters, Julia, « Ananda Devi as Transcolonial Translator », Intimate Enemies.

Translation in Francophone Contexts, Francophone Postcolonial Studies, 4, 2013, p. 216-234.

\section{Mémoires et thèses sur les romans d'Ananda Devi consultés}

Alis, Celine, Water, Walls and Dreams: Society and Poetics of Space in At the Other End of Myself by Marie-Therese Humbert, Pagli by Ananda Devi, and Blue Bay Palace by Nathacha Appanah, thèse, 233 pages, Louisiana, University of Louisiana at Lafayette, 2009.

Appadoo, Sheila, Etude comparative du personnage féminin dans les œuvres d'Ananda Devi et de Marie-Thérèse Humbert, mémoire, 70 pages, Massachusetts, Brandeis University, 1995.

Bannerjee, Reena, La Construction identitaire dans l'œuvre romanesque d'Ananda Devi, thèse, 172 pages, Ontario, University of Western Ontario, 2008.

Githire, Njeri, Voices from Ex/Isle: Caribbean and Indian Ocean Women Writers Break Geographical Confines, thèse, 259 pages, Pennsylvania, The Pennsylvania State University, 2004.

Kistnareddy, Ashwini, "Hybridity" in the Novels of Ananda Devi, mémoire de DEA, 242 pages, Nottingham, The University of Nottingham, 2011.

Lindo, Karen, States of Shame: Women, Affect, Transnationalisms, thèse, 295 pages, Los Angeles, University of California, 2007.

Mahatma, Maitryee, Sitā et ses doubles : mythes et représentations dans les æuvres d'Ananda Devi, thèse, 232 pages, Paris, université Paris XIII, 2008.

Meur, Marie-Caroline, Identité et monstruosité chez Ananda Devi, thèse, 346 pages, Paris, université Paris IV, Sorbonne, 2009.

Ramsey, Karen, L'Ecriture d'Ananda Devi : du cloisonnement à une proposition d'ouverture interculturelle, mémoire de DEA, 145 pages, St-Denis, université de la Réunion, 2005.

Rosnay de, Formet, Le Discours de l'impasse. Allegories de l'assujettissement dans L'Avalée des avalées et Moi, l'interdite, mémoire, 72 pages, Kingston, Queen's University, 2012.

Vaitanaki, Eva, Sacrifice, aliénation et reconquête, itinéraire de deux personnages féminins d'Ananda Devi dans Le voile de Drapaudi, Pagli, mémoire de DEA, 124 pages, St-Denis, université de la Réunion, 2005. 
Ziethen, Antje, Géo/Graphies : la poétique de l'espace (post)colonial dans le roman sénégalais et mauricien au féminin, thèse, 241 pages, Toronto, University of Toronto, 2010.

\section{Ouvrages sur la littérature consultés}

Anonyme, « Mauritius: the road to Independence (1945- 1968) », http://www.eisa.org.za/WEP/mauoverview7.htm, consulté le 22 mars 2013.

Anyinefa, Koffi, « Le Métro parisien : figure de l'exotisme postcolonial », French Forum, vol. 28, 2003, p. 77-98.

Arrivé, Michel, « Toponymie et littérature », http://hal.inria.fr/docs/00/82/29/75/PDF/TOPONYMIE_LITTA_RATURE_PD F.pdf, consulté le 1 mai 2014.

Arnold, Marcus, «Les Hiérarchies socio-économiqueset ethniques à l'île Maurice : homogénéités et ruptures dans l'identité des Franco-Mauriciens », Nouvelles études francophones, 26, 2011, p. 125-141.

Auerbach, Erich, Mimesis. The Representation of Reality in Western Literature, Oxford, Princeton University Press, 1953.

Bachelard, Gaston, La Poétique de l'espace, Paris, Presses Universitaires de France, 1970.

Baissac, C., Etude sur le patois créole mauricien, Genève, Slatkine Reprints, 1976.

Baron, Christine, «Littérature et géographie : lieux, espaces, paysages et écritures », http://www.fabula.org/lht/8/baron.html, consulté le 29 avril 2014.

Barthes, Roland, «L’Effet de réel », Communications, 11, 1968, p. 84-89.

Barthes, Roland, «Writing and Revolution », Yale French Studies, 39, 1967, p. 77-84.

Bellet, Roger, « Jules Vallès, journaliste devant le roman réaliste (1864-1865) », Revue des sciences humaines, 119, 1965, p. 353-385.

Beniamino, Michel, La Francophonie littéraire. Essai pour une théorie, Paris, L'Harmattan, 1999.

Bertrand, Denis, « De la topique à la figuration spatiale », http://revues.unilim.fr/nas/document.php?id=2759Denis Bertrand, consulté le 27 février 2013.

Bongie, Chris, «Edouard Glissant: Dealing in Globality », Postcolonial Thought in the French-Speaking World, Liverpool, Liverpool University Press, 2009.

Borowski, Mateusz, Malgorzata Sugiera, Fictional Realities/Real Fictions. Contemporary Theatre in Search of a New Mimetic Paradigm, Newcastle, Cambridge Scholars Publishing, 2007.

Boucher, Geneviève, «Espace littéraire et spatialisation de la littérature », p. 80-85, https://uottawa.scholarsportal.info/ojs/index.php/revueanalyses/article/viewFile/690/591, consulté le 23 avril 2014.

Boutaghou, Maya, " "Défense et illustration" d'un universel mauricien », International Journal of Francophone Studies, 13, 2010, p. 451-469. 
Bovet, Ernest, «Le Réalisme de Falubert », Revue d'histoire littéraire de la France, 1, 1911, p. 1-36.

Bragard, Véronique, «Transoceanic Echoes: Coolitude and the Work of the Mauritian Poet Khal Torabully », International Journal of Francophone Studies, 8, 2005, p. 219-233.

Brown, Tony, David Williams, James Jackson, Harold Neighbors, Myriam Torres, Sherrill Sellers, Kendrick Brown, « Being Black and Feeling Blue: the Mental Health Consequences of Racial Discrimination », Race \& Society, 2, 2000, p. $117-131$.

Brumm, Ursula, « Thoughts on History and the Novel », Comparative Literature Studies, 6, 1969, p. 317-330.

Bunwaree, Sheila, «The Marginal in the Miracle : Human Capital in Mauritius », International Journal of Educational Development, 21, 2001, p. 257-271.

Burns, Lorna, «Becoming-Postcolonial, Becoming-Caribbean: Edouard Glissant and the Poetics of Creolization », Textual Practice, 23, 1, p. 99-117.

Butor, Michel, «La Ville comme texte», dans Répertoire V et dernier, Paris, Editions de Minuit, p. 33-36.

Byrne Francis, Thom Huebner, Development and Structures of Creole Languages. Essays in Honor of Derek Bickerton, Amsterdam, John Benjamins Publishing Company, 1991.

Calvet, Louis-Jean, La Guerre des langues et politiques linguistiques, Paris, Payot, 1987.

Carter, Marina, Khal Torabully, Coolitude. An Anthology of the Indian Labour Diaspora, London, Anthem Press, 2002.

Champfleury, Le Réalisme, Genève, Slatkine Reprints, 1967.

Champion, Henry, The Great Dock Strike: in London, August, 1889, London, Swan Sonnenschein, 1890.

Charon, Joel, The Meaning of Sociology, Englewood Cliffs, Prentice-Hall, 1987.

Chauderson, Robert, Les Créoles français, Paris, Fernand Nathan, 1979.

Cobb, Martha, « Ortiz, Glissant, and Ellison: Fictional Patterns in Black Literature », Afro-Hispanic Review, 21, 2002, p. 179-184.

Cohn, Dorrit, The Distinction of Fiction, London, The Johns Hopkins University, 1999.

Currie, Gregory, « Fictional Truth », Philosophical Studies. An International Journal for Philosophy in the Analytic Tradition, 50, 1986, p. 195-212.

De la Hoz, Rafael, Jaime Szeinuk, «Longshoremen, Stevedores and Dockworkers », http://toxicology.ws/Greenberg/Chapter\%2021\%20-

\%20Longshoremen,\%20Stevefores,\%20and\%20Dockworkers.pdf, consulté le 21 janvier 2014.

Dédéyan, Charles, «Balzac dans "le littérateur universel" », Acta Baltica: Liber Annalis Instituti Baltici, 1, 1980, p. 239-244.

Deloughrey, Elizabeth, « On Kala Pani and Transoceanic Fluids », New Literature Review, 47, 2011, p. 71-92. 
Donnellan, Keith, «Speaking of Nothing », The Philosophical Review, 83, 1974, p. 331.

Dornel, Laurent, «Les Usages du racialisme. Le cas de la main-d'œuvre coloniale en France pendant la Première Guerre mondiale », Genèses, 20, 1995, p. 48-72.

Dubreuil, Laurent, « Don du français et parole (post)coloniale », International Journal of Francophone Studies, 10, 2007, p. 345-358.

Dumont, Micheline, «Romans historiques : l'histoire n'est pas une appellation contrôlée », LE DEVOIR.com-Libre de penser, 2006, http://www.ledevoir.com/non-classe/104093/romans-historiques-l-histoire-nest-pas-une-appellation-controlee, consulté le 13 mars 2012.

Dunn, Ernest, « Critical perspectives: Local, Regional, Universal? », African Literature Today, 4, 1978, p. 52-55.

Durkheim, Emile, The Division of Labour in Society, London, Macmillan Publishers, 1984.

Durkheim, Emile, Le Suicide. Etude de sociologie. Livre premier. Les facteurs extrasociaux, http://classiques.uqac.ca/classiques/Durkheim_emile/suicide/suicide_Livre_1.p df, consulté le 16 décembre 2013.

Durkheim, Emile, Le Suicide. Etude de sociologie. Livre deuxième. Causes sociales et types sociaux, http://classiques.uqac.ca/classiques/Durkheim emile/suicide/suicide Livre 2. pdf, consulté le 8 janvier 2014.

Durkheim, Emile, Le Suicide. Etude de Sociologie. Livre troisième. Du suicide comme phénomène social en général, http://classiques.uqac.ca/classiques/Durkheim_emile/suicide/suicide_Livre_3. pdf, consulté le 9 janvier 2014.

Eco, Umberto, « Reading My Readers », Comparative Literature, 107, 1992, p. 819827.

Eco, Umberto, « Lector in Fabula », The Threepenny Review, 48, 1992, p. 5.

El Nossery, Névine, Témoignages fictionnels au féminin. Une réécriture des blancs de la guerre civile algérienne, New York, Rodopi, 2012.

English, Philip, «Mauritius Reigniting the Engines of Growth. A Teaching Case Study », http://siteresources.worldbank.org/WBI/Resources/wbi37136.pdf, consulté le 2 septembre 2013.

Eriksen, Thomas, « Politics, Jobs and Marriages: Ethnic Relations in Mauritius », http://folk.uio.no/geirthe/Equality.html, consulté le 2 septembre 2013.

Eriksen, Thomas, "Creolization in Anthropological Theory and in Mauritius », http://folk.uio.no/geirthe/Creolisation.pdf, consulté le 14 janvier 2014.

Fletcher, Lisa, « ... Some distance to go », New Literary Review, 47, 2011, p. 17-34.

Formigari, Lia, «Herder entre universalisme et singularité », Revue germanique internationale, 20, 2003, p. 133-143. 
Gefen, Alexandre, «Le Vraisemblable comme vérisimilitude », Atelier de théorie littéraire, 2007, http://www.fabula.org/atelier.php?Le_vraisemblable_comme_v\%26eacute\%3Br isimilitude, consulté le 5 septembre 2011.

Ghose, Zulfikar, The Fiction of Reality, London, The Macmillan Press, 1983.

Gibson, Warren, «Unemployment: What is it? », A Journal for Western Man, issue cccv, 2011, p. 17-20.

Girmitunited.org, « Colonial British Indian Indentured Labour Transportation by Country », http://girmitunited.org/girmit/?page_id=816, consulté le 13 novembre 2013.

Goodman, Morris, A Comparative Study of Creole French Dialects, London, Mouton, 1964.

Gour, Clive, Représentation du créole et thématique identitaire dans quelques œuvres littéraires d'expression française postindépendance à Maurice, mémoire, 250 pages, Ottawa, université d'Ottawa, 2008.

Grignon, Claude, Jean-Claude Passeron, Le Savant et le populaire. Misérabilisme et populisme en sociologie et en littérature, Paris, Gallimard, Le Seuil, 1971.

Guellouz, Suzanne, «Une fiction qui fait l'histoire : la relation historique et galante de l'invasion de l'Espagne par les Arabes de Baudot de Juilly (1699) », Travaux de littérature, 23, 2010, p. 105-114.

Hawkins, Peter, The Other Hybrid Archipelago, Plymouth, The Rowan and Littlefield Publishing Group, 2007.

Hospers, John, « Truth and Fictional Characters », Journal of Aesthetic Education, 14, 1980, p. 5-17.

Halen, Pierre, « Moura, Jean-Marc. La littérature des lointains. Histoire de l'exotisme européen au $\mathrm{XX}^{\mathrm{e}}$ siècle », Cahiers d'études africaines, http://etudesafricaines.revues.org/37?lang=en, consulté le 7 juillet 2013.

Hertel, François, « Du misérabilisme intellectuel, du besoin de se renier... et de quelques "chefs-d'œuvre" », http://bibnum2.banq.qc.ca/bna/actionnationale/src/1967/04/13/1967-04-13.pdf, consulté le 20 août 2013.

Heyer den, Kent, «Configuring Historical Facts through Historical Fiction: Agency, Art-in-Fact, and Imagination as Stepping Stones between Then and Now », Educational Theory, 57, 2007, p. 141-157.

Hughes, Susan, «Expletives of Lower Working-Class Women », Language in Society, 21, 1992, p. 291-303.

Jacob, Happymon, «Home and Displacement: Refugees, Diaspora and India », http://sas.sagepub.com/content/11/1/100.full.pdf+html, consulté le 10 mars 2014.

Jones, C. Grahame, «Quelques observations sur le conflit entre le réalisme et la fantaisie dans le roman québécois », Revue d'histoire littéraire du Québec et du Canada, 13, 1987, p. 137-153.

Jouve, Vincent, « Pour une analyse de l'effet-personnage », Littérature, 85, 1992, p. $103-111$. 
Jouve, Vincent, L'Effet-Personnage dans le roman, Paris, Presses Universitaires de France, 1992.

Kadel, Yusuf, MondesFrancophones.com: revue mondiale des francophonies, « Île Maurice : repousser continuellement les limites de la poésie », 2011, http://mondesfrancophones.com/espaces/afriques/intervention-de-yusuf-kadeldans-le-cadre-de-la-conference-animee-par-le-professeur-benjamin-beniaminoa-1\%E2\%80\%99universite-de-limoges-sur-la-litterature-mauricienne-1/, consulté le 2 mai 2012.

Kapor, Vladimir, « Couleur locale. A Pictoral Term Gone Astray? », A Journal of Verbal/Visual Enquiry, 25, 2008, p. 22-32.

Karlsen Saffron, James Nazroo, « Relation between Racial Discrimination, Social Class, and Health among Ethnic Minority Groups », American Journal of Public Health, 92, 2002, p. 624-631.

Kee Mew, Evelyn, «La Littérature mauricienne et les débuts de la critique », International Journal of Francophone Studies, 13, 2010, p. 417-433.

Kee Mew, Evelyn, «Le Mauricianisme et l'indianocéanisme : deux projets de définition de la littérature mauricienne », http://revel.unice.fr/symposia/actel/?id=441, consulté le 15 juillet 2014.

King, Richard, « The Discipline of Fact/The Freedom of Fiction? », Journal of American Studies, 25, 1991, p. 171-188.

Kitsuse, John, « Societal Reaction to Deviant Behaviour: Problems of Theory and Method », Social Problems, 9, 1962, p. 247-256.

Kourouma, Ahmadou, «La Littérature francophone retrouve sa place », Courrier International, 2008, http://www.courrierinternational.com/article/2008/07/23/lalitterature-francophone-retrouve-sa-place, consulté le 13 mai 2012.

Kriegel, Sibylle, Ralph Ludwig, Fabiola Henri, « Encoding Path in Mauritian Creole and Bhojpuri. Problems of Language Contact», http://www.llf.cnrs.fr/Gens/Henri/encoding-path.pdf, consulté le 14 janvier 2014.

Lange, Matthew, «Embedding the Colonial State. A Comparative Historical Analysis of State Building and Broad-Based Development in Mauritius », Social Science History, 27, 2003, p. 397-423.

Le Bozec, Yves, «Le Vrai et le vraisemblable. Rhétorique et poétique », Revue des sciences humaines, 280, 2005.

Levine, Bruce, « Mental Illness or Rebellion », Ethical Human Psychology and Psychiatry, 7, 2005, p. 125-172.

Lévy, Bertrand, « Géographie et Littérature. Une synthèse historique », Le Globe, 146, 2006, p. 25-52.

L'Express, « Aslakha Callikhan-Proag explique Marcel Cabon », http://www.lexpress.mu/node/192113, consulté le 15 juillet 2014.

Lionnet, Françoise, «Lire l'exotisme by Jean-Marc Moura », The French Review, 71, 1997, p. 98-99.

Lionnet, Françoise, Postcolonial Representations. Women, Literature, Identity, London, Cornell University Press, 1995. 
Lionnet, Françoise, Le Su et l'incertain. Cosmopolitiques créoles de l'océan Indien, Trou d'Eau Douce, île Maurice, 2012.

Lionnet, Françoise, Ecritures féminines et dialogues critiques. Subjectivité, genre et ironie, Trou d'Eau Douce, île Maurice, 2012.

Lionnet, Françoise, « Matière à photographie : cosmopolitique et modernité créoles à l'île Maurice », French Forum, 34, 2009, p. 75-99.

Loichot, Valérie, «We Are all Related: Edouard Glissant Meets Octavia Butler », Small Axe, 30, 2009, p. 38-51.

Magdelaine-Andrianjafitrimo, Valérie, «Fantasme d'une terre matricielle : la représentation de l'Inde dans les romans francophones de l'engagisme aux Antilles et dans l'océan Indien », Synergies Inde, 1, 2006, p. 207-220.

Magdelaine-Andrianjafitrimo, Valérie, «Une mise en scène de la diversité linguistique : comment la littérature francophone mauricienne se dissocie-t-elle des nouvelles normes antillaise ? », Revue de sociolinguistique en ligne, 3 , 2004, p. 142-165.

Mathy, Jean-Philippe, "Refonder l'universalisme” : Bourdieu, Balibar et 1'"exception" philosophique française ", Contemporary French and Francophone Studies, 12, 2008, p. 357-368.

Maupassant, Guy de, «Le Roman », dans Pierre et Jean, Paris, Albin Michel, 1970, p. $829-842$.

McCarthy, Mary, « The Fact in Fiction », Partisan Review, 27, 1960, p. 438-458.

McCusker, Maeve, Anthony Soares, Islanded Identities. Constructions of Postcolonial Cultural Insularity, New York, Rodopi, 2011.

McKee Michael, Ian Robertson, Social Problems, New York, Random House, 1975.

Mehta, Brinda, «Indianités francophones : Kala Pani Narratives », L'Esprit créateur, 50, 2010, p. 1-11.

Meltzer, Bernard, Richard Musolf, « Resentment and Ressentiment », Sociological Inquiry, 72, 2002, p. 240-255.

Merton, Robert, Social Theory and Social Structure, New York, The Free Press, 1968.

Merton, Robert, « Social Structure and Anomie », American Sociological Review, 3, 1938, p. 672-682.

Michel, Andreas, «En route to the Other : Victor Segalen's Essai sur l'exotisme and Equipée », Romance Studies, 16, 1990, p. 21-30.

Mikkonen, Kai, « Can Fiction Become Fact? The Fiction-to-Fact Transition in Recent Theories of Fiction », Style, 40, 2006, p. 291-313.

Miroslav, Volf, « Nothing but the Truth », The Christian Century, 120, 2003, p. 33.

Mitterand, Henri, «L'Espace du corps dans le roman réaliste », dans Au bonheur des mots : mélanges en l'honneur de Gérald Antoine, Nancy, Presses universitaire de Nancy, 1984, p. 341-335.

Moss, Anita, «A Feminist Study of Mystic Structures », Children's Literature Association Quarterly, 7, 1982, p. 19-20. 
Moura, Jean-Marc, «L'Exotisme fin-de- $\left(\mathrm{XX}^{\mathrm{e}}\right)$ siècle », Revue de littérature comparée, 4, 2000, p. 533-594.

Muratore, M.J., « Historical Imposters, Fictional Truths: La Princesse de Clèves », A Quarterly Journal in Modern Literatures, 54, 2001, p. 245-258.

Nardout-Lafarge, Elisabeth, «Instabilité du lieu dans la fiction narrative contemporaine », temps zéro, http://tempszero.contemporain.info/document974, consulté le 22 avril 2014.

Niess, Robert, J., «Emile Zola: from Fact to Fiction », Modern Language Notes, 63, 1948, p. 407-408.

Nojgaard, Morten, « Le Problème du réalisme dans les romans de Marivaux. Réflexions sur l'introduction de la voiture embourbée », Revue romane, 1966, p. 71-87, http://img.kb.dk/tidsskriftdk/pdf/rro/rro_0001PDF/rro 0001 95132.pdf, consulté le 22 septembre 2011.

Office of the United Nations Resident Coordinator, « Common Country Assessment.Mauritius », http://www.law.yale.edu/rcw/rcw/jurisdictions/afe/mauritius/mauritius_assessm ent.pdf, consulté le 3 septembre 2013.

Olivieri-Godet, Rita, «Les Fils hybrides du tissage de l'histoire dans $O$ nobre sequestrador de Antonio Torres », http://www.antoniotorres.com.br/internacional9.html, consulté le 19 décembre 2012.

Orrù, Marco, Anomie, London, Allen \& Unwin, 1987.

O’Toole, Lawrence, « Dimensions of Semiotic Space in Narrative », Poetics Today, 4, 1980, p. 135-149.

Packham, Catherine, « Feigning Fictions: Imagination, Hypothesis and Philosophical Writing in the Scottish Enlightenment », The Eighteenth Century, 48, 2007, p. $149-171$.

Palmer, Frank, Literature and Moral Understanding. A Philosophical Essay on Ethics, Aesthetics, Education and Culture, Oxford, Oxford University Press, 1992.

Pannetier-Manckoundia, Florence, « Revisiting the Universal: Wilson Harris and Edouard Glissant », The Global and the Particular in the English-Speaking World, 2002, p. 79-87.

Philophil.com, «La Représentation de la femme en sorcière », http://www.philophil.com/philosophie/representation/Analyse/sorciere.htm, consulté le 2 mai 2012.

Pihlainen, Kalle, « The Moral of the Historical Story: Textual Differences in Fact and Fiction », New Literary History, 33, 2002, p. 39-60.

Poddar, Namrata, « The Islands Within: Alterity, Imperialism and the Métroport in Mauritian Fiction », Dalhousie French Studies, 94, Spring 2011, p. 39-51.

Port-Louis City Landslide Protection Project, http://www.jica.go.jp/english/our work/evaluation/oda loan/post/2002/pdf/136 _full.pf, consulté le 3 septembre 2013.

Pratt, Annis, « Women and Nature in Modern Fiction », Contemporary Literature, 13, 1972, p. 467-490. 
Prieto, Eric, «Edouard Glissant, Littérature-monde, and Tout-monde », Small Axe, 33, 2010, p. 111-120.

Putnam, Hilary, « Realism and Reason », Proceedings and Addresses of the American Philosophical Association, 50, 1977, p. 483-498.

Radnika, Dan, « 100 Years Ago: the Philadelphia Dockers Strike and Local 8 of the IWW », http://libcom.org/library/100-years-ago-philadelphia-dockers-strikelocal-8-iww-mouvement-communiste-kolektivn\%C4\%9B-pr, consulté le 12 novembre 2013.

Ramharai, Vicram, «Entre littérature mauricienne et littérature francophone : quels enjeux pour les écrivains mauriciens ? », Postcolonial Literatures of the Francophone Indian Ocean, e-France: an online Journal of French Studies, 2, 2008, p. 20-38, http://www.reading.ac.uk/web/FILES/e-france/Ramharai.pdf, consulté le 2 mai 2012.

Ramharai, Vicram, «La Littérature mauricienne d'expression anglaise : problèmes et perspectives », Journal of Mauritian Studies, 2, 1988, p. 1-39.

Ramsden, Maureen A., « Fictional Frontiers: the Interrelation of Fact and Fiction between the World and the Text », Neophilologus, 95, 2011, p. 341-358.

Repp, Charles, « Justification from Fictional Narratives », The Journal of Aesthetic Education, 48, 2014, p. 25-44.

Reuschel Anne-Kathrin, Hurni Lorenz, « Mapping Literature: Visualisation of Spatial Uncertainty in Fiction », The Cartography Journal, 48, 2011, p. 293-308.

Richard, François, «Le Décor romanesque », Etudes françaises, 8, 1972, p. 343-362.

Ricœur, Paul, «Can Fictional Narratives Be True?», dans The Phenomenology of Man and of the Human Condition. Individualisation of Nature and the Human Being, Dordrecht, Reidel Publishing Company, 1983, p. 3-19.

Riffaterre, Michael, Fictional Truth, London, The John Hopkins University Press, 1990.

Rizi, Elaheh, Salehi, «Les jeux entre la réalité et la fiction dans le roman : on s'y fera de Zoyâ Pirzâd », http://revel.unice.fr/symposia/actel/index.html?id=364,consulté le 20 août 2014.

Ronen, Ruth, « Space in Fiction », Poetics Today, 7, 1986, p. 421-438.

Ryan, Marie-Laure, Possible Worlds, Artificial Intelligence and Narrative Theory, Indiana, University of Bloomington and Indianapolis Press, 1991.

Sainsbury, Mark, Fiction and Fictionalism. New Problems of Philosophy, London, Routledge, 2010.

Sample Donald, C., Evans, «Estimates of the Infection Rates for Poliomyelitis Virus in the Years Preceding the Poliomyelitis Epidemics of 1916 in New York and 1945 in Mauritius », The Journal of Hygiene, 55, 1957, p. 254-265.

Sanderson, Stephen, Macrosociology. An Introduction to Human Societies, New York, Harper \& Row Publishers, 1988.

Sauerberg, Lars, « Fact-Flirting Fiction: Historiographical Potential or Involuntary Parody? », European Journal of English Studies, 3, 1999, p. 190-205. 
Schapira, Charlotte, «Maupassant et le fait divers », Hebrew University Studies in Literature and Arts, 14, 1987, p. 23-32.

Schlick, Rachel, Essay on Exoticism. An Aesthetics of Diversity. Victor Segalen, London, Duke University Press, 2002.

Schuhl, Pierre-Maxime, « Gaston Bachelard et la "Poétique de l'Espace", Revue philosophique de la France et de l'étranger, 148, 1958, p. 212-214.

Scott, Carolyn, Marjorie Zatz, « Comparative Deviance and Criminology », International Journal of Comparative Sociology, xxii, 1981, p. 237-256.

Seidl, Ivan, « Aspects de l'espace dans le roman français moderne », www.phil.muni.cz/plonedata/wurj/erb/.../8seidl-74.rtf, consulté le 23 avril 2014, p. 121-130.

Sellers, Robert, Cleopatra Caldwell, Karen Schmeelk-Cone, Marc Zimmerman, «Racial Identity, Racial Discrimination, Perceived Stress, and Psychological Distress among African American Young Adults », Journal of Health and Social Behavior, 44, 2003, p. 302-317.

Soh, Kelvin, TIMELINE: Key dates in the history of the personal computer, http://www.reuters.com/article/2009/01/07/us-laptop-sbidUSTRE50601V20090107, consulté le 15 novembre 2013.

Sombo, N'Cho, Dominique Tabutin, «Tendances et causes de la mortalité à Maurice depuis $1940 »$, Population, 40, 1985, p. 435-453.

Spivak, Gayatri Chakravorty, A Critique of Postcolonial Reasons. Toward a History of the Vanishing Present, London, Harvard University Press, 1999.

Subramanian, Arvind, «L'île Maurice. Etude de cas », Finance et Développement, 38, 2001, p. 22-25.

Thibault, Bruno, J.M.G. Le Clézio et la métaphore exotique, New York, Rodopi, 2009.

Tibloux, Emmanuel, « Les Enjeux littéraires de la description de l'espace », Espace Temps, 62-63, 1996, p. 116-129.

Timol, Umar, « Dynamisme littéraire à Maurice, leurre ou lueur? », Revues mondiales des Francophones, 2011,

http://mondesfrancophones.com/espaces/afriques/interventions-de-yusuf-kadelet-umar-timol-dans-le-cadre-de-la-conference-animee-par-le-professeurbenjamin-beniamino-a-1\%e2\%80\%99universite-de-limoges-sur-la-litteraturemauricienne/, consulté le 29 juillet 2011.

Todorov, Tzvetan, Nous et les autres. La réflexion française sur la diversité humaine, Paris, Seuil, 1989.

Todorov, Tzvetan, Poétique de la prose, Paris, Seuil, 1971.

Tranquille, Danielle, «Inscriptions of Dev/Fiancé: métissage in Mauritian Literature », International Journal of Francophone Studies, 8, 2005, p. 199-218.

Truth \& Justice Commission, « Report of the Truth and Justice Commission. Volume $1 »$, http://www.usip.org/sites/default/files/ROL/TJC_Voll.pdf, consulté le 3 mars 2013 . 
Truth \& Justice Commission, « Report of the Truth and Justice Commission. Volume 3. Contemporary History, Culture and Society. Research Reports, Technical Studies and Surveys », http://pmo.gov.mu/English/Documents/TJC_Vol3.pdf, consulté le 13 mars 2014.

Truth \& Justice Commission, « Report of the Truth and Justice Commission. Volume 4. History, Economy, Society and Memory. Research Reports, Technical Studies and Surveys », http://pmo.gov.mu/English/Documents/TJC Vol4modified.pdf, consulté le 30 mars 2014.

Ungurianu, Dan, « Fact and Fiction in the Romantic Historical Novel », The Russian Review, 57, 1998, p. 380-393.

Valdman, Albert, Initiation à la linguistique. Le Créole : structure, statut et origine, Paris, Editions Klincksieck, 1978.

Vernier-Larochette, Béatrice, «La Logique de la création dans L'Euvre de Zola. Fiction ou réalité ?», Initiales, 18, 1999, p. 23-41.

Viera, Domingos, « Miguel Torga : "L'universel, c'est le local moins les murs" », Diasporiques, 9, 2010, p. 46-50.

Weede Erich, Edward Muller, « Rebellion, Violence and Revolution: a Rational Choice perspective », Journal of Peace Research, 35, 1998, p. 43-59.

Westphal, Bertrand, La Géocritique. Réel, fiction, espace, Paris, Les Editions de Minuit, 2007.

White, Thornton, A Master Plan for Port Louis. Mauritius, Cape Town, Cape Times, 1952.

Wokler, Robert, « Todorov's Otherness », New Literary History, vol. 27, 1996, p. 4355.

World Health Organization, « Consequences of Alcohol Use. Social problems with Alcohol Use », http://www.who.int/substance abuse/publications/globalstatusreportalcohol200 4_socproblems.pdf, consulté le 22 janvier 2014.

Ziethen, Antje, «La Littérature et l'espace », Arborescences : revue d'études françaises, 3, 2013, p. 3-29.

Zippel, Frank, « Unreliable Narration and Fictional Truth », Journal of Literary Theory, 5, 2011, p. 109-130.

\section{Ouvrages sur l'histoire de l'île Maurice consultés}

Aapravasi Ghat World Heritage, «The Genesis of Immigration »,

http://www.aapravasighat.org/indenture.htm, consulté le 12 avril 2012.

Aapravasi Ghat, «Un expatrié français à l'île Maurice », 2010, http://expatfrancais.services-ile-maurice.com/tag/coolies, consulté le 3 mai 2012.

Aapravasi GhatWorld Heritage, « Aapravasi Ghat Trust Fund Newsletter », 1, 2008, p. 1-16.

Aapravasi GhatWorld Heritage, « Aapravasi Ghat Trust Fund Newsletter », 1, 2009, p. $1-24$. 
Aapravasi GhatWorld Heritage, « Aapravasi Ghat Trust Fund Newsletter », 1, 2010, p. $1-24$.

Abdullah, Zain, «African "Soul Brothers" in the Hood: Immigration, Islam, and the Black Encounter», Anthropological Quarterly, 82, 2009, p. 37-62.

Addison J., K. Hazareesing, A New History of Mauritius, Mauritius, Editions de l'océan Indien, 1989.

Agrawal, Baleshwar, « Mauritius. My Second Home », Mother India. Children Abroad, 2, 1988, p. 34-41.

Allen, Richard, « Capital, Illegal Slaves, Indentured Labourers and the Creation of a Sugar Plantation Economy in Mauritius, 1810-1860 », The Journal of Imperial and Commonwealth History, 36, 2008, p. 151-170.

Allen, Richard, B., Labour History of Mauritius Slaves, Freedmen and Indentured Labourers in Colonial Mauritius, Cambridge, Cambridge University Press, 1999.

Allen, Richard, B., «Licentious and Unbridled Proceedings: the Illegal Slave Trade to Mauritius and the Seychelles during the Early Nineteenth Century », The Journal of African History, 42, 2001, p. 91-116.

Anderson, Clare, Convicts in the Indian Ocean: Transportation from South Asia to Mauritius, 1835-1853, London, Macmillan, 2000.

Anderson, Clare, « The Politics of Punishment in Colonial Mauritius, 1766-1887 », Cultural and Social History, 5, 2008, p. 411-422.

Anderson, Clare, «Convicts and Coolies: Rethinking Indentured Labour in the Nineteenth Century », A Journal of Slave and Post-Slave Studies, 30, 2009, p. 93-109.

Anderson, Clare, «Indian Convict Ship Mutinies in the Mid-Nineteenth Century », http://www.sasnet.lu.se/EASASpapers/22ClareAnderson.pdf, consulté le 11 septembre 2013.

Anderson, Clare, Subaltern Lives. Biographies of Colonialism in the Indian Ocean World, 1790-1920, Cambridge, University Press, 2012.

Anonyme, «Port-Louis sous le signe de la violence. Mesures policières à l'étude », Le Cernéen, $\mathrm{n}^{\mathrm{0}}$ 5261, 20 janvier 1968.

Anonyme, « Port-Louis. La situation s'aggrave », Le Cernéen, n 5262, 22 janvier 1968.

Anonyme, « Port-Louis. L'ordre doit être rétabli. Des mesures draconiennes s'imposent », Le Cernéen, nº 5263, 23 janvier 1968.

Anonyme, « Port-Louis encore sous l'emprise de la peur hier », Le Cernéen, n 5264, 24 janvier 1968.

Anonyme, «La Vague de violence à Port-Louis. La Riot Unit et la S.M.F prêtes à intervenir », Week-end, nํ 69, 21 janvier 1968.

Anonyme, « 20 morts, 212 blessés. Le bilan d'une semaine de violence », Week-end, $\mathrm{n}^{\mathrm{o}} 70,28$ janvier 1968.

Anonyme, «Grave incendie aux Dockers' Flats, Roches Bois. Un enfant de deux ans meurt. Ses parents dans un état critique », Le Dimanche, $\mathrm{n}^{\mathrm{0}}$ 1.00, 18 janvier 1981. 
Araujo, Ana, «Welcome the Diaspora: Slave Trade Heritage Tourism and the Public Memory Slavery », Ethnologies, 32, 2010, p. 145-157.

Asgarally, Issa, « Histoire et linguistique : langues, colonialisme et sousdéveloppement. Tentative de description de la superstructure linguistique de l'île Maurice de 1721-1982 », Journal of Mauritian Studies, 1, 1986, p. 130147.

Bahadur, Gaiutra, Coolie Woman. The Odyssey of Indenture, Chicago, The University of Chicago Press, 2014.

Bahadur, Gaiutra, « Coolie Women Are in Demand Here », The Virginia Quarterly Review, 87, 2, 2011, http://www.vqronline.org/essay/coolie-women-aredemand-here, consulté le 9 septembre 2014.

Bissessur, Raj, " Chômage : quand la femme est victime », http://www.defimedia.info/defi-quotidien/dq-societe/item/57712-chomagequand-la-femme-est-victime.html, consulté le 13 août 2014.

Bissondoyal U., S. Servansing, «Indian Labour Immigration », Papers presented at the International Conference on Indian Labour Immigration, Mahatma Gandhi Institute, 1986.

Bissondoyal. U., Indians overseas. The Mauritian Experience, Mauritius, Mahatma Gandhi Institue Press, 1984.

Bissondoyal. B., The Truth about Mauritius, Bombay, India, Bhartiya Vidya Bhavan, 1968.

Boni, Tanella, « Humanity, Inhumanity and Closeness in the Look », Diogenes, 49, 2002, p. 57-65.

Boudet, Catherine, «Emeutes et élections à Maurice. La mort de Kaya, aléa ou échec de la construction nationale ? », Politique africaine, 79, 2000, p. 153-164.

Bragard, Véronique, Transoceanic Dialogue, Coolitude in Caribbean and Indian Ocean Literatures, coll. Comparatism and Society, vol. 5, Brussels, Peter Lang, 2008 .

Bragard, Véronique, «Transoceanic Echoes: Coolitude and the Work of the Mauritian poet Khal Torabully », International Journal of Francophone Studies, 8, 2005, p. 219-233.

Bragard, Véronique, «Gendered Voyages into Coolitude: the Shaping of the IndoCaribbean Woman's Literacy Consciousness », Kunapipi: Journal of PostColonial Writing, 20, 1998, p. 99-111.

Bragard, Véronique, «L'Empreinte des Kalapani dans la littérature caribéenne et mauricienne : une comparaison transcoloniale », L'Esprit créateur, 50, 2010, p. 86-94.

Britannica. Academic Edition, « Aryan », http://www.britannica.com/EBchecked/topic/37468/Aryan, consulté le 28 août 2014.

Brodwin, Paul, « Marginality and Cultural Intimacy in a Transnational Haitian Community », Occasional Paper, 91, 2001, p. 1-36.

Burrun, Breejan, Port-Louis-Mauritius, China, Christian Le Comte, 2009. 
Carroll Barbara, Terrance Carroll, « Trouble in Paradise: Ethnic Conflict in Mauritius », Commonwealth and Comparative Politics, 38, 2008, p. 25-50.

Carter, Marina, Voices from Indenture. Experiences of Indian Migrants in the British Empire, London, Leicester University Press, 1996.

Carter M., J. Ng, Forging the Rainbow-Labour Immigrants in British Mauritius, Mauritius, Alfran, 1997.

Chatterjee, Rajeswari, A History of the People of the Subcontinent of India in a Nutshell, Nevada, Frandsen Humanities Press, 2003.

Collen, Lindsey, « A Page in Revolutionary History. The 1979 General Strike in Mauritius », http://directaction.org.au/issue14/a page in revolutionary history the 1979 ge neral_strike_in_mauritius, consulté le 05 novembre 2012.

Collen, Lindsey, « Another Side of Paradise », http://newint.org/features/2009/05/01/mauritius-class/, consulté le 18 septembre 2013.

Commons and Lords Hansard, Commons Sitting of 23 January 1968, « Mauritius (State of Emergency) », http://hansard.millbanksystems.com/commons/1968/jan/23/mauritius-state-ofemergency, consulté le 30 août 2013.

Cumpston, M., «A Survey of Indian Immigration to British Tropical Colonies to $1910 »$, Population Studies, 10, 1956, p. 158-165.

Cuniah, C., «Coolitude », http://www.radiomoris.com/forum/discussionsgenerales/5343-que-pensez-vous-de-la-coolitude.htm, consulté le 3 décembre 2013.

Curpen, Indradev, « Workers' Struggle Is a Never Ending Process », http://www.defimedia.info/news-sunday/society/item/10857-michel-gerardnina-workers\%E2\%80\%99-struggle-is-a-never-endingprocess.html?tmpl=component\&print, consulté le 26 mars 2013.

Daby, Pardeep Kumar, « Les grèves en 1971, c'était pour notre dignité », http://www.defimedia.info/dimanche-hebdo/dh-people/item/38006-govinsamycarpen-ex-employes-des-docks-les-greves-en-1971-c-etait-pour-notredignite.html, consulté le 2 septembre 2013.

De Smith, Stanley, « Mauritius: Constitutionalism in a Plural Society », The Modern Law Review, 31, 1968, p. 601-622.

Deerpalsing S., M. Carter, V. Govinden, V. Teelock, Selected Documents on Indian Immigration. Volume I. (Mauritius 1834-1926). Organisation and Evaluation of the Indenture System, Mauritius, Mahatma Gandhi Institute press, 1994.

Deerpalsing S., Kwong Ng Foong J., « End of Indenture », dans Labour Immigrants in Mauritius. A Pictorial Recollection, Mauritius, Mahatma Gandhi Institute Press, 2001.

Deloughrey, Elizabeth, « On Kala Pani and Transoceanic Fluids », New Literature Review, 47, 2011, p. 71-92.

Dewoo, Teena, «The Coolitude of Coolietude: the $(\mathrm{Re})$ negotiation of the Indian Identity in Mauritius », Postambule, 8, 2012, p. 1-21. 
Dikhira, Chit, History of Mauritius. Experiments in Democracy, Delhi, India, Brijbasi Arts Press, 2002.

Dubey, Ajay, Government and Politics in Mauritius, India, Kalinge Publications, 1997.

Electoral Institute for Sustainable Democracy in Africa, « Mauritius: the Road to Independence (1945-1968) », http://www.content.eisa.org.za/oldpage/mauritius-road-independence-1945-1968, consulté le 1 juillet 2014.

Elyulph, Stanley, « The Treatment of Indian Immigration in Mauritius », Fortnightly Review, 17, 1875, p. 794-819.

Faruqee, Ashrufa, « Conceiving the Coolie Woman: Indentured Labour, Indian Women and Colonial Discourse », South Asia Research, 16, 1996, p. 61-76.

Forman, Ross, « Coolie Cargoes: Emigrant Ships and the Burden of Representation in Joseph Conrad's Typhoon and James Dalziel's Dead Reckoning », English Literature in Transition, 47, 2004, p. 398-428.

Fortuné, Joyce, Labourers, Dockers and Zenfan Balye. A Report on Workers in the Buffer Zone, Mauritius, Aapravasi Ghat Trust Fund, 2006.

Graham, Scott, « Gandhi's Salt March to Dandi », http://postcolonialstudies.emory.edu/ghandis-salt-march-to-dandi/, consulté le 17 septembre 2013.

Hayley, Julia, « No Hope in Spain for African Boat People », The Guardian, http://global.factiva.com.helicon.vuw.ac.nz/ha/default.aspx, consulté le 24 septembre 2013.

Houbert, Jean, « Décolonisation en pays créole : l'île Maurice et La Réunion », http://horizon.documentation.ird.fr/exldoc/pleins_textes/pleins_textes_7/polaf/p df/010078.pdf, consulté le 29 octobre 2013.

Houbert, Jean, « Mauritius: Independence and Dependence », The Journal of Modern African Studies, 19, 1981, p. 75-105.

Howard-Hassmann, Rhoda, « Reparations for the Slave Trade: Rhetoric, Law, History and Political Realities », Canadian Journal of African Studies, 41, 2007, p. 427454.

Hooker, Juliet, « Indigenous Inclusion/Black Exclusion: Race, Ethnicity and Multicultural Citizenship in Latin America », Journal of Latin American Studies, 37, 2005, p. 285-310.

Hooper, Charles Arthur, Report of the Commission of Enquiry into Unrest on Sugar Estates in Mauritius, 1937, Port-Louis, Government Printer, 1938.

Industrial Workers of the World, « The General Strike for Industrial Freedom », http://www.iww.org/search/node/the\%20general\%20strike\%20for\%20industrial \%20freedom, consulté le 20 mai 2014.

International Labour Office, Dock Work. Social Repercussions of New Methods of Cargo Handling, Geneva, International Labour Office, 2002.

Jacob, Happymon, « Home and Displacement: Refugees, Diaspora and India », http://sas.sagepub.com/content/11/1/100.full.pdf+html, consulté le 1 décembre 2013. 
Jean-François, Emmanuel Bruno, «La Représentation de la traversée des eaux par les esclaves et les engagés dans les littératures francophones contemporaines de l'île Maurice et de Madagascar », Journal of Mauritian Studies, 1, 2009, p. 117129.

Jeetah, Ramkissoon, «Indians in Mauritius. 1910-1988», Mother India. Children Abroad, 2, 1988, p. 16-26.

Kaur, Ramandeep, « Who were the Dravidians in India? », http://www.mapsofindia.com/my-india/history/who-were-dravidians-in-india, consulté le 28 août 2014.

LabourStart, «Global New ITUC Global Rights Index. The World's Worst Countries for Workers », http://www.labourstart.org/2013/, consulté le 21 mai 2014.

Lal, Brij, «The Odyssey of Indenture: Fragmentation and Reconstitution in the Indian Diaspora », A Journal of Transnational Studies, 5, 1996, p. 167-188.

Le Defi Plus, « Histoire-1979 : les grévistes à deux doigts de la mort », http://www.defimedia.info/defi-plus/dp-societe/item/57259-histoire-1979-lesgrevistes-a-deux-doigts-de-la-mort.html, consulté le 3 août 2014.

L'Express, « Adieu les Dockers' Flat», http://www.lexpress.mu/node/216368, consulté le 10 mai 2008.

Le Morne Heritage Trust Fund, « Slavery and Marooning », http://www.gov.mu/portal/sites/lemorne/history.htm\#MaroonsandLeMorne, consulté le 15 novembre 2013.

Macmillan, Allister, Mauritius Illustrated. Historical and Descriptive, Commercial and Industrial, Facts, Figures and Resources, Londres, 1914.

Mangerel, Caroline, «La Drive, le marronnage : présentation d'un mode d'errance insulaire et créole », Nouvelles études francophones, 25, 2010, p. 90-106.

Marks, Kathy, « City Life. Port-Louis, Mauritius: Melting Pot that Is Starting to Boil over », The Independent. Foreign News, 1999, p. 12.

Mauritiusmag, « An Eye Witness Account of the 1968 Riots », http://www.mauritiusmag.com/?p=598, consulté le 30 août 2013.

Maurer, Sylvie, « Former British Colony: Mauritians in the Face of Globalisation », Ontrocom Online Journal of Anthropology, 7, 2011, p. 235-250.

McDougall, Julie, Don Fletcher, « Dehumanising the Boat People », Social Alternative, 21, 2002, p. 33-36.

Mishra, Amit, «Indian Indentured Labourers in Mauritius », Studies in History, 25, 2009, p. 229-251.

Mishra, Amit, From Indentured Labour to Liberated Nation. Public Policy and Small Planters in Mauritius, India, Focus Press, 2007.

Mishra, Amit, Mauritius, Delhi, National Book Trust, 2009.

Mookherji, S., The Indenture System in Mauritius (1837-1915), Calcutta, Firma K.L. Mukhopadhyay, 1962.

Moutou, Benjamin, L'île Maurice. Vingt-cinq leçons d'histoire (1598-1998), RicheTerre, Maurice, Alfran, 1998. 
Mozaffar, Shaheen, « Negotiating Independence in Mauritius », International Negotiation, 10, 2005, p. 263-291.

Napal, Dayachand, British Mauritius 1810-1948, Mauritius, Intellectual Publishing Company, 1984.

New Zealand History Online, « The 1951 Waterfront Dispute », http://www.nzhistory.net.nz/politics/the-1951-waterfront-dispute, consulté le 1 mai 2014.

Northrup, David, Indentured Labour in the Age of Imperialism, 1834-1922, Cambridge, Cambridge University Press, 1995.

Peerthum, Satyendra, «The Horror of Slave Voyage to Mauritius Remembered », http://www.lexpress.mu/article/horror-slave-voyage-mauritius-remembered, consulté le 24 septembre 2013.

Peerthum, Satyendra, « History and Remembrance. "An Untold Story": the Arrival of Indentured Labourers in Mauritius between 1826 and $1834 »$, http://www.lemauricien.com/article/history-remembrance-\%E2\%80\%9Cuntold-story\%E2\%80\%9D-arrival-indentured-labourers-mauritius-between1826-and-, consulté le 19 novembre 2013.

Peerthum, Satyendra, «The Port-Louis Dockers Strike of 1938 », http://www.lexpress.mu/article/port-louis-dockers\%E2\%80\%99-strike-1938, consulté le 19 novembre 2013.

Plevitz, Loretta de, « Arthur Hamilton Gordon and Adolphe de Plevitz: Ambitions for Indian Labour in Colonial Mauritius and Fiji », Queensland History Journal, 21, 2010, p. 181-196.

Prosper, Jean-Georges, Histoire de la littérature mauricienne de langue française, Rose-Hill, Editions de l'océan Indien, 1978.

Prosper, Jean-Georges, L'île Maurice : au sommet de la vague économique francophone, Paris, L’Harmattan, 1993.

Pydayya, Ramasawmi, Kalla, Indians in Mauritius, Delhi, Star Publications, 1936.

Quenette, Rivaltz, La Corporation municipale de Port-Louis. Volume IV. Le retour au scrutin de liste 1924-1935, Port-Louis, Super Printing Company, 2002.

Sagar, Vidya, «Indian Migration to Mauritius: 1834-1910 », Mother India. Children Abroad, 2, 1988, p. 9-15.

Saturday Review of Politics, Literature, Science and Art, « Chinese Coolie in Cuba », British Periodicals, 43, 1877, p. 77-78.

Scarr, Deryck, Slaving and Slavery in the Indian Ocean, Great Britain, Macmillan Press, 1998.

Seegobin, Ram, « Hunger Strikes: "Food refusal" as a Means of Struggle », http://www.lalitmauritius.org/viewnews.php?id=965, consulté le 4 septembre 2013.

Selvon, Sydney, A Comprehensive History of Mauritius, from Beginning to 2001, PortLouis, Mauritius, Mauritius Printing Specialists, 2001.

Sibeud, Emmanuelle, «Post-Colonial et Colonial Studies : enjeux et débats », Revue d'histoires moderne et contemporaine, 51, 2004, p. 87-95. 
Slusser, George, « History, Historicity, Story », Science Fiction Studies, 15, 1988, p. 187-213.

Special Mobile Force, «Presentation of Regimental Colour on the Occasion of the $50^{\text {th }}$ Anniversary of the SMF », SMF Gymkhana Grounds, Vacoas.

Stiebel, Lindy, «Crossing the Kala Pani: Cause for "Celebration" or "Commemoration" 150 year on? Portrayals of Indenture in Recent South African Writing », Journal of Literary Studies, 27, 2011, p. 77-89.

Storey, Kelleher, « Small-Scale Sugar Cane Farmers and Biotechnology in Mauritius: the "Uba" Riots of 1937 », Agricultural History, 69, 1995, p. 163-176.

Swift Lowe, Candice, «Privileging the Diaspora in Mauritius: Making World Heritage for a Multicultural Nation », Diaspora. A Journal of Transnational Studies, 16, 2007, p. 287-323.

Teelock, Vijaya, «Breaking the Wall of Silence: Slavery in Mauritian Historiography », Radical History Review, 91, 2005, p. 104-109.

Teelock, Vijaya, «The Influence of Slavery in the Formation of Creole Identity », Comparative Studies of South Asia, Africa and the Middle East, 19, 1999, p. 38.

Teelock, Vijaya, Mauritian History. From its Beginning to Modern Times, Mauritius, Mahatma Gandhi Institute Press, 2009.

The National Archives, «Indian Identured Labourers », 2007, http://www.nationalarchives.gov.uk/records/research-guides/indian-indenturedlabour.htm, consulté le 5 juillet 2012.

The Irish Times, « Deaths Toll in Lampedusa Boat Tragedy Rises to 232 », http://www.irishtimes.com/news/world/europe/death-toll-in-lampedusa-boattragedy-rises-to-232-1.1553814, consulté le 16 novembre 2013.

The Mauritius Sugar Bulk Terminal Corporation, « The Mauritius Sugar Bulk Terminal Corporation », http://prb.pmo.gov.mu/English/Pages/prb\%202003/Parastatal\%20and\%20Other \%20Statutory\%20Bodies/Mauritius-Sugar-Terminal-Corporation.aspx, consulté le 3 septembre 2013.

Tinker, Hugh, A New System of Slavery, London, The Camelot Press, 1974.

Torabully, Khal, « Esclaves et coolies : pour un rapprochement des mémoires », Africultures, 67, 2006, p. 101-109.

Torabully, Khaleel, « The Coolies' Odyssey », The Unesco Courrier, 10, 1996, p. 1316.

Torabully, Khal, «A l'Aapravasi Ghat, île Maurice. Dialogue enfin avec l'histoire », http://www.montraykreyol.org/spip.php?article1657, consulté le 10 décembre 2013.

Torabully, Khal, «Créolité, coolitude, créolisation : les imaginaires de la relation », http://www.luxiotte.net/entretiens/torabully1.htm, consulté le 3 décembre 2013.

Torabully, Khal, « Maurice. Plus qu'une juxtaposition, il nous faut un itinéraire double d'une passerelle entre l'Aapravasi Ghat et Le Morne », http://www.montraykreyol.org/spip.php?article1378, consulté le 10 décembre 2013. 
Toussaint, Augustin, Port-Louis. Deux siècles d'histoire (1735-1935), Port-Louis, Maurice, La Typographie Moderne, 1936.

Urena-Rib, Pedro, « Créolités dans les Amériques », Nouvelles études francophones, 25, 2010, p. 107-125.

Varma, Moonindra, The Political History of Mauritius. Volume I (1883-1983). Recollections and Reflections, Mauritius, Bahadoor Printing, 2011.

World Heritage List, « Le Morne Cultural Landscape », http://whc.unesco.org/en/list/1259, consulté le 19 juin 2014.

World Socialist Web Site, « Workers Struggles: Asia, Australia and the Pacific », http://www.wsws.org/en/articles/2008/10/labo-o11.html, consulté le 20 décembre 2014.

\section{Ouvrages sociologiques consultés}

Abraham, Marie, «L'île Maurice : source inépuisable d'inspiration », Salon du Livre de l'outre-mer, $6^{\text {ème }}$ éd., 1999, p. 10-11.

Adey Shaen, Fiona McIntosh, Sept jours à l'île Maurice et Rodrigues, Le Cap, New Holland Publishing, p. 25, 2004.

Afriquinfos, «Corruption : Maurice chute de neuf places à l'indice de Transparency International », http://www.afriquinfos.com/articles/2013/12/4/corruptionmaurice-chute-neuf-places-lindice-transparency-international-237620.asp, consulté le 27 février 2014.

Agamben, Giorgio, Michel Boccara, «L'Homme et l'animal », 2002, http://www.globenet.org/transversales/grit/animal.htm, consulté le 2 mai 2012.

Agathe, Ludovic, « 45 ans de l'indépendance et un mauricianisme qui se cherche encore », http://www.lexpress.mu/article/45-ans-de1\%E2\%80\%99ind\%C3\% A9pendance-et-un-mauricianisme-qui-se-chercheencore, consulté le 13 janvier 2014.

Agenda News, « Mauritius: Trauma for Young Lesbians », http://www.afrika.no/Detailed/12586.html, consulté le 24 mars 2014.

AllAfrica.com, « Port-Louis. Capitale délaissée », http://search.proquest.com/docview/1018104942?accountid=14782, consulté le 13 novembre 2013 .

Amnesty International, « Making Love a Crime: Criminalization of Same-Sex Conduct in Sub-Saharan African », http://www.amnesty.org/en/library/asset/AFR01/001/2013/en/9f2d91b7-bc0e4ea7-adae-7e51ae0ce36f/afr010012013en.pdf, consulté le 24 mars 2014.

Anderson, Clare, «Subaltern Lives : History, Identity and Memory in the Indian Ocean World », History Compass, 11, 2013, p. 503-507.

Anonyme, «Adieu les Dockers' Flat », http://www.lexpress.mu/services/archive107739-adieu-les-dockers-flat.html, consulté le 10 mai 2011.

Anonyme, « Ports and Harbours », http://www.pfri.uniri.hr/ bopri/documents/14-MEtal_001.pdf, consulté le 1 mai 2013. 
Anonyme, « Mauritius Reptiles », http://www.mauritius-reptiles.com/the-reptiles-ofmauritius/introduced-reptiles/house-geckos/, consulté le 21 février 2013.

Anonyme, «Demographics of Mauritius », http://en.wikipedia.org/wiki/Demographics_of_Mauritius, consulté le 11 septembre 2013.

Arno, Toni, Ile Maurice. Une société multiraciale, Paris, L'Harmattan, 1986.

Asgarally, Issa, « Histoire et linguistique : langues, colonialisme et sousdéveloppement. Tentative de description de la superstructure linguistique de l’île Maurice de 1721 à 1982 », Journal of Mauritian Studies, 1, 1986, p. 130147.

Aumeerally, N.L., " "Tiger in paradise": Reading Global Mauritius in Shifting Time and Space », Journal of African Cultural Studies, 17, 2005, p. 161-180.

Barrault, Michèle, Ile Maurice. Regards, Michel Hetier, Mauritius, 1990.

Barrere, Claude, « Les langues », 2001, http://barrere.claude.free.fr/maurice/langues.htm, consulté le 2 mai 2012.

Belk, Russel, «Pimps for Paradise : Missionaries, Monetary Funds and Marketers », Marketing Intelligence \& Planning, 18, 2000, p. 337-343.

Beniamino, Michel, «Écritures féminines à l'île Maurice : une rupture postcoloniale ? », Nouvelles études francophones, 23, 1, 2008, p. 145-153.

Bhattacharya, Shilpi, « The Desire for Whiteness : Can Law and Economics Explain it? », Columbia Journal of Race and Law, 2, 2011, p. 117-147.

Boswell, Rosabelle, «Le Malaise créole: Ethnic Identity in Mauritius », dans New Directions in Anthropology, New York, Oxford, Berghahn, 2006.

Boswell, Rosabelle, « Heritage Tourism and Identity in the Mauritian Villages of Chamarel and Le Morne », 31, 2005, p. 283-295.

Boutia, Mario, «Au cœur de l'info : la hausse dans les délits sexuels inquiète », http://www.defimedia.info/defi-quotidien/dq-actualites/item/58097-au-coeurde-l-info-la-hausse-dans-les-delits-sexuels-inquiete.html, consulté le 19 août 2014.

Bragard, Véronique, « Matière à photographie : cosmopolitiques et modernité créoles à l'île Maurice », French Forum, 34, 2009, p. 75-99.

Bragard, Véronique, Srilata Ravi, Ecritures mauriciennes au féminin : penser l'altérité, Paris, L'Harmattan, 2011.

Brow, Tony, David Williams, James Jackson, Harold Neighbours, Myriam Torres, Sherrill Sellers, Kendrick Brown, «Being Black and Feeling Blue : the Mental Health Consequences of Racial Discrimantion », Race \& Society, 2, 2000, p. 117-131.

Brown, W. Norman, «La Vache sacrée dans la religion hindoue », Annales, Économies, Sociétés, Civilisations, 19, 1964, p. 643-664.

Bunwaree, Sheila, « Economics, Conflicts and Interculturality in a Small Island State: the Case of Mauritius », Polis/R.C.S.P/C.P.S.R, 9, 2002, p. 1-19.

Bunwaree, Sheila, « Growth, Gender and Equity NEPAD and Renaissance: Myth or Reality?», Economica, 16, 2009, p. 1-15. 
Bunwaree, Sheila, « Small Island Developing States: Challenges, Prospects and International Cooperation for Sustainable Development », IRFD World Forum, http://irfd.org/events/wfsids/virtual/papers/sids_sbunwaree.pdf, consulté le 13 décembre 2013.

Bureau of Democracy, Human Rights and Labour, « 2013 Human Reports Rights: Mauritius », http://www.state.gov/j/drl/rls/hrrpt/2013/af/220139.htm, consulté le 24 mars 2014.

Burton, Benedict, « Mauritius at the Crossroads », The British Journal of Sociology, 12, 1961, p. 387-392.

Cap Soleil, « Paille-en-queue. Phaeton lepturus », http://www.capsoleilmaurice.com/fr/les-oiseaux/paille-en-queue.html, consulté le 24 janvier 2013.

Carmignani, Sandra, «Figures identitaires créoles et patrimoine à l'île Maurice : une montagne en jeu », Journal des anthropologues, 2006, p. 265-285.

Carpenter, Belinda, « The Prostitute and the Client : Challenging the Dualisms », Women's Studies International Forum, 21, 1998, p. 387-399.

Charles, Jean-Claude, «Ile Maurice, une société de décadence », http://www.defimedia.info/blog/item/2357-\%C3\%AEle-maurice-unesoci\%C3\%A9t\%C3\%A9-ded\%C3\%A9cadence.html?tmpl=component\&print=1, consulté le 27 février 2014.

Chazan-Gillig, Suzanne, «The Roots of Mauritian Multi-Culturalism and the Birth of a New Social Contract: Being “Autochtone”, Being Creole », Journal of Mauritian Studies, 2, 2003, p. 64-86.

Chazan-Gillig, Suzanne, «Insularité et mondialisation. Diasporas et créolisation de la société mauricienne contemporaine », Journal des anthropologues, 96-97, 2004, p. 321-339.

Chazan-Gillig, Suzanne, «Ethnicity and Free exchange in Mauritian Society », Social Anthropology, 8, 2000, p. 33-44.

Chemla, Yves, « Des voyages hors de soi dont on ne revient pas... », 2007, Côté Sud, http://homepage.mac.com/chemla/fic_doc/Devi_121007.html, consulté le 8 mai 2011.

Chiba, Eugene, « English in Mauritius », http://homes.chass.utoronto.ca/ cpercy/courses/6362-chiba.htm, consulté le 25 février 2014.

Chowdhury, Amitava, « Towards an Archaeological Heritage Management of Aapravasi Ghat », Journal of Mauritian Studies, 2, 2003, p. 87-104.

Christopher, A.J., «Ethnicity, Community, and the Census in Mauritius, 1830-1990 », The Geographical Journal, 158, 1992, p. 57-64.

Claveyrolas, Mathieu, «L'ancrage de l'hindouisme dans le paysage mauricien : transfert et appropriation », Autrepart, 56, 2010, p. 17-38.

Cohen, Barney, «Small Cities, Big Problems », Science and Technology, 20, 2004, p. 87-89.

Collins, Randall, «The Micro Contribution to Macro Sociology », Sociological Theory, 6, 1988, p. 242-253. 
Conseil du statut de la femme, «La Prostitution : profession ou exploitation ? Une réflexion à poursuivre », http://collections.banq.qc.ca/ark:/52327/bs52753, consulté le 23 janvier 2014.

Convention on the Elimination of all Forms of Discrimination against Women, «Concluding Observations of the Committee on the Elimination of Discrimination against Women: Mauritius », http://www2.ohchr.org/english/bodies/cedaw/docs/co/CEDAW-C-MUS-CO-67.pdf, consulté le 25 mars 2014.

Cook, Brian, George Winokur, «Alcoholism as a Family Dysfunction », Psychiatric Annals, 23, 1993, p. 508-512.

Cornille, Jean-Louis, « Mémoires d'océan et d'ailleurs : traversées interocéaniques », Tydskrif vir letterkunde, 50, 2013, p. 86-96.

Creig, Alastair, «The Fragility of Success: Repositioning Mauritian Development in the Twenty-First Century », Island Studies Journal, 6, 2011, p. 157-178.

Crowley, Eve, «Creoles », New Encyclopedia of Africa, 2, 2008, p. 528-532.

Cunniah, C., «Coolitude », Mauritius Mag: History, Society, Culture and People of Mauritius, L'Express, 2010, http://www.mauritiusmag.com/?p=198, consulté le 1 mai 2012.

Cunniah, Bruno, Shakuntala Boolell, Fonction et représentation de la Mauricienne dans le discours littéraire, Rose-Hill, Mauritius Printing Specialists, 2000.

Daby, Pradeep, « Des Dockers Flats aux nouvelles résidences : ce qui a changé pour des familles entières », http://www.defimedia.info/defi-plus/dpsociete/item/55709-des-dockers-flats-aux-nouvelles-residences-ce-qui-achange-pour-des-familles-entieres.html, consulté le 8 juin 2014.

Dickens, Charles, «Londoners over the Border », Household Words, 16, 1857, p. 241244.

Dictionnaires des symboles, «le symbolisme du coq », http://www.dictionnairedessymboles.fr/article-le-symbolisme-du-coq61928839.html, consulté le 1 août 2014.

Divya, A. «Why Sati is Still a Burning Issue », The Times of India, http://timesofindia.indiatimes.com/home/stoi/Why-sati-is-still-a-burningissue/articleshow/4897797.cms?, consulté le 12 août 2014.

Dorsey M., P. Ingram, « Guide to Exploring African American Culture », http://pubs.cas.psu.edu/FreePubs/pdfs/agrs92.pdf, consulté le 15 février 2014.

Duntley J., D. Buss, « Homicide Adaptations », Aggression and Violent Behaviour, 16, 2011, p. 399-410.

Dupon, J.F., «La Société mauricienne », Revue juridique et politique, 23, 1969, p. 337356.

Durkheim, Emile, The Rules of Sociological Methods, New York, The Free Press, 1982.

Edensor, Tim, « Caudan », dans Small Cities. Urban Experience beyond the Metropolis, Oxon, Routledge, 2006, p. 205-216. 
Ensminger, Margaret, David Celentano, « Gender Differences in the Effect of Unemployment on Psychological Distress », Social Science and Medicine, 30, 1990, p. 469-477.

Eisenlohr, Patrick, « Creole Publics: Language, Cultural Citizenship and the Spread of the Nation in Mauritius », Comparative Studies in Society and History, 49, 2007, p. 968-998.

Eriksen, Thomas, Hylland, «Tu Dimunn Pu Vinn Kreol: the Mauritian Creole and the Concept of Creolization », 1999, http://folk.uio.no/geirthe/Creoles.html, consulté le 13 avril 2012.

Eriksen, Thomas, Hylland, « We and Us: Two Modes of Group Identification », Journal of Peace Research, 32, 1995, p. 427-436.

Fernandez, Bina, N. B. Gomathy, « The Nature of Violence Faced by Lesbian Women in India »,http://download.tiss.edu/fap/RCI-VAW/RCI-

VAW_Publications/The_Nature_of_violence_faced_by_Lesbian_women_in_In dia.pdf, consulté le 21 mars 2014.

François, Paul, « La communauté créole de l'île Maurice », http://csmsmagazine.org/?p=1667, consulté le 29 octobre 2013.

Garfield, Eugene, « The Anomie. Deviant Behaviour Connection: the Theories of Durkheim, Merton and Srole », 10, 1987, p. 272-281.

Gelles, Rebecca, «Fair and Lovely: Standards of Beauty, Globalization, and the Modern Indian Woman », http://digitalcollections.sit.edu/cgi/viewcontent.cgi?article=2143\&context=isp_c ollection, consulté le 13 février 2013.

Gerbeau, Hubert, « Religion et identité créole à l'île Maurice », Histoire, Monde et Culture religieuse, 12, 2009, p. 53-71.

Ghasarian, Christian, «La Mise en scène de la possession », 2002, Mardévirin, http://www.indereunion.net/IREV/ghasarian/da2.htm, consulté le 3 novembre 2011.

Goldo, Rak, «Les Herbes médicinales et magiques du Moyen âge », http://racinescharnelles.blogspot.co.nz/2010/12/les-herbes-medicinales-etmagiques-du.html, consulté le 3 mai 2013.

Goon P., A. Craven, «Whose debt?: Globalisation and Whitefacing in Asia », http://intersections.anu.edu.au/issue9/gooncraven.html, Intersections: Gender, History and Culture in the Asian Context, 9, 2003.

Gosai, Anjana, «India's Myth of Fair-Skinned Beauty », http://www.theguardian.com/lifeandstyle/2010/jul/19/india-fair-skinned-beauty, consulté le 14 février 2014.

Green, Maia, « Representing Poverty and Attacking Representations: Perspectives on Poverty from Social Anthropology », Journal of Development Studies, 42, 2006, p. 1108-1129.

Gregory, Marilyn, « Masculinity and Homicide-Suicide », International Journal of Law, Crime and Justice, 40, 2012, p. 133-151.

Gujarati in Mauritius, http://joshuaproject.net/people groups/11982/MP, consulté le 1 avril 2014. 
Hiltebeitel, Alf, The Cult of Draupadi 2. On Hindu Ritual and the Goddess, Chicago and London, The University of Chicago Press, 1991.

Hollander, Jason, « Renowned Columbia Sociologist and National Medal of Science Winner Robert K. Merton Dies at $92 »$, http://www.columbia.edu/cu/news/03/02/robertKMerton.html, consulté le 12 décembre 2013.

Hollup, Oddvar, « The Disintegration of Caste and Changing Concepts of Indian Ethnic Identity in Mauritius », Ethnology, 33, 1994, p. 297-316.

Indianastrology2000.com, « General Results of Being Born in Chitra Nakshatra », 2010, http://www.indianastrology2000.com/astrology-clues/chitra.php, consulté le 13 mars 2012.

Institut national de la santé et de la recherche médicale, "Anorexie », La santé de l'homme, 2008, http://www.inserm.fr/thematiques/neurosciences-sciencescognitives-neurologie-psychiatrie/dossiers-d-information/anorexie, consulté le 13 mars 2012.

Islam K., H. Ahmed, E. Karim, A. Amin, « Fair Factor. The Whiter the Better », http://archive.thedailystar.net/magazine/2006/05/02/cover.htm, consulté le 14 février 2014.

Jackson, Joan, «Alcoholism and Family », Annals of the American Academy of Political and Social Science, 315, 1958, p. 90-98.

Jean-François, Emmanuel Bruno, «L'expérience de la violence dans le roman mauricien francophone de la nouvelle génération », International Journal of Francophone Studies, vol. 13, 2010, p. 513-529.

Jheelan, Daniel, « Fruits of Mauritius », http://dodoilemaurice.free.fr/litchi.htm, consulté le 12 mars 2013.

Jones, Frank, « Micro-Macro Linkages in Sociological Analysis: Theory, Method and Substance », Journal of Sociology, 31, 1995, p. 74-92.

Joseph, Lauren, Pamela Black, «Who’s the Man? Fragile Masculinities, Consumer Masculinities, and the Profiles of Sex Work Clients », Men and Masculinities, 15, 2012, p. 486-506.

Karsten Paul, Klaus Moser, « Unemployment Impairs Mental Health: Meta-Analyses », Journal of Vocational Behavior, 74, 2009, p. 264-282.

Kasenally, Roukaya, « Mauritius: Paradise Reconsidered », Journal of Democracy, 22, 2011, p. 160-169.

Kattany, Nilen, «Bilan du judiciaire pour 2013 : les femmes demandent le divorce plus que les hommes », http://www.defimedia.info/live-news/item/53682-bilan-dujudiciaire-pour-2013-les-femmes-demandent-le-divorce-plus-que-leshommes.html, consulté le 31 mai 2013.

Katyal, Neal, «Men Who Own Women: a Thirteenth Amendement Critique of Forced Prostitution », The Yale Law Journal, 103, 1993, p. 791-826.

Khodabocus, Nooreeda, «ENN SEL LEPEP, ENN SEL NATION ? : communalisme vs. mauricianisme », http://www.lepep.net/2013/03/enn-sel-lepep-enn-selnation-communalisme-vs-mauricianisme/, consulté le 13 janvier 2013. 
Kiesling, Scott, « Power and the Language of Men », The Masculinities Reader, USA, Malden, 2001, p. 65-85.

Kubrin, Charis, Ronald Weitzer, « New Directions in Social Disorganization Theory », Journal of Research in Crime and Delinquency, 40, 2003, p. 374-402.

Kumar, Virendra, « Burnt wives. A Study of Suicides », Burns, 29, 2003, p. 31-35.

Lallah, Pravesh, «ICAC: Ten Years on. Why We Are Still the Champions of Fraud and Corruption », http://www.lemauricien.com/article/icac-ten-years-\%E2\%80\%93why-we-are-still-champions-fraud-and-corruption, consulté le 27 février 2014.

Larousse, « Le Port », http://www.larousse.fr/dictionnaires/francais/port/62676?q=le+port\#61970, consulté le 3 mai 2013.

Leroueil, Emmanuel, «Le Miracle mauricien », http://terangaweb.com/le-miraclemauricien/, consulté le 14 janvier 2014.

L'Express, Archive, «L'échappée nostalgique d'Eileen Lohka », http://www.lexpress.mu/services/archive-44987-1-echappee-nostalgique-deileen-lohka.html, consulté le 11 avril 2012.

Le Mauricien, «SRILATA RAVI : la littérature mauricienne n'est pas bornée sur un héritage fixe, elle déborde cette île », http://www.lemauricien.com/article/srilata-ravi-la-litterature-mauricienne-npas-bornee-sur-heritage-fixe-elle-deborde-ile, consulté le 21 mai 2013.

LGBT Asylum News, «Gays in Mauritius Are Kidnapped to "Cure" their Homosexuality », http://sdgln.com/causes/2010/08/25/gays-mauritius-arekidnapped-cure-their-homosexuality\#sthash.ptF5ObuT.dndyNnUw.dpbs, consulté le 23 mars 2014.

Lionnet, Françoise, « Matière à photographie, cosmopolitique et modernité créoles à l'île Maurice », French Forum, 34, 3, 2009, p. 75-99.

Lionnet, Françoise, Thomas Spear, «Introduction: Mauritius in/and Global Culture : Politics, Literature, Visual Arts », International Journal of Francophone Studies, 13, 2010, p. 371-400.

Lincoln, David, « Labour Migration in the Global Division of Labour: Migrant Workers in Mauritius », International Migration, 47, 2009, p. 129-156.

Lindo, Karen, « Mapping Territories of Affective Communites in the Indian Ocean », International Journal of Francophone Studies, 13, 2010, p. 471- 488.

Linfo.Re, Antenne Réunion Télévision, « Hausse du nombre de divorces à Maurice », http://www.linfo.re/049-Ocean-Indien/402-Societe/496065-Hausse-du-nombrede-divorces-a-Maurice, consulté le 3 mars 2014.

Lohka, Eileen, «Lectures de l'écriture mauricienne à la lueur des penseurs canadiens », http://revel.unice.fr/symposia/actel/index.html?id=424, consulté le 3 juin 2014.

Longéras, Stéphanie, «La culture créole a besoin de retrouver ses valeurs », Témoignages. Festival International Kréol de Maurice, 2006, http://www.temoignages.re/la-culture-creole-a-besoin-de,18976.html, consulté le 14 mars 2012. 
Lowe, Candice, Indrani Gopauloo, Colette Le Chantier, Diana Bablee, Le Morne Cultural Landscape. History, Symbolism and Tradition, Port-Louis, Mauritius, Government Printing Department, 2010.

Lutchmaya, Jane, «Violence domestique : quand la répression ne suffit pas ! », http://www.defimedia.info/defi-quotidien/dq-actualites/item/49078-violencedomestique-quand-la-repression-ne-suffit-pas.html, consulté le 21 mars 2014.

Magedera, Ian, « France-India-Britain, (Post)Colonial Triangles : Mauritius/India and Canada/India, (Post)Colonial Tangents », International Journal of Francophone Studies, 5, 2002, p. 64-73.

Mahadeo, Satish, « History of English and French Languages in Mauritius: a Study in Languages and Power », http://www.educ.utas.edu.au/users/tle/journal/articles/Mahadeo/Mahadeo4.ht $\underline{\text { ml}}$, consulté le 25 février 2014.

Mamet, Joseph, «L'île Maurice et sa civilisation. Histoire : les langues à Maurice », 1949, p. 383, http://www.cjp.net/macnews/JuneJuly10.pdf, consulté le 3 mai 2012.

Marypages, «Les apparitions de la Vierge à Fatima », 2011, http://www.marypages.com/fatimaFrench.htm, consulté le 13 mars 2012.

McGinley, William, « Reduction in Sociology », Philosophy of Social Sciences, 42, 2012, p. 370-398.

McDonnell, Lawrence, «Skin Color and the Census. Slave Bodies », United States Bureau of the Census, 2002, p. 114-117.

Mearns, Andrew, The Bitter Cry of Outcast London. An Inquiry into the Condition of the Abject Poor, London, James Clarke, 1883.

Miles, William F.S., « The Creole Malaise in Mauritius », Oxford Journals, 98, 1999, p. 211-228.

Miley, James, « Critical Dimensions in Human Ecology: Ideology in American Sociology », Journal of Contemporary Ethnography, 9, 1980, p. 163-185.

Milrod C., R. Weitzer, «The Intimacy Prism: Emotion Management among the Clients of Escorts », Men and Masculinities, 15, 2012, p. 447-467.

Ministry of Gender Equality, Child Development and Family Welfare, « Statistics in Mauritius. A Gender Approach. February $2013 »$, http://gender.gov.mu/English/Documents/Statistics\%20in\%20Mauritius\%20\%20A\%20Gender\%20Approach\%2024.07.13.pdf, consulté le 21 mars 2014.

Ministry of Health and Quality of Life, « Dr. A. Gaffoor. Jeetoo Hospital», http://www.gov.mu/portal/site/mohsite/menuitem.4581ded5607bad351a42860a a0208a0cl, consulté le 12 mai 2014.

Ministry of Finance and Economic Development, « 2011 Housing and Population Census, Mauritius. Volume 2. Demographic and Fertility Characteristics », http://www.gov.mu/portal/goc/cso/file/2011VolIIPC.pdf, consulté le 8 juin 2014.

Mohanty, Manoj, Manoj Panigrahi, Sachidananda Mohanty, Das Srimanta, «Victimiologic Study of Female Homicide », Legal Medecine, 6, 2004, p. 151156. 
Moss, Roger, «Ennti-liv: Publication in the Margins », Wasafiri, 17, 2002, p. 37-41.

Monto M., N. McRee, «A Comparison of the Male Customers of Female Street Prostitutes with National Samples of Men », International Journal of Offender Therapy and Comparative Criminology, 49, 2005, p. 505-528.

Moonean, Jenilaine, « Droits de la communauté LGBT. A la recherche du temps perdu », http://www.defimedia.info/defi-quotidien/dq-societe/item/9995-droitsde-la-communaut\%C3\%A9-lgbt-\%E2\%80\%93-\%C3\%A0-la-recherche-dutemps-perdu.html, consulté le 7 juin 2014.

Morey, Robert, « Hinduism Exposed », http://njiat.com/JunePDFs/Hinduism\%20Exposed\%2003 01 09.pdf, consulté le 15 février 2014.

Mulloo, Anand, « Hindus in Mauritius », Mother India. Children Abroad, 2, 1988, p. 69-76.

Nave, Ari, « The Role of Marriages in the Maintenance of Ethnic Group Boundaries: the Case of Mauritius », http://arinave.com/arinavec/wpcontent/uploads/2012/05/pan-african1.pdf, consulté le 28 février 2014.

Nave, Ari, « Marriage and the Maintenance of Ethnic Group Boundaries: the Case of Mauritius », Ethnic and Racial Studies, 23, 2000, p. 329-352.

Nave, Ari, « Nested Identities: Ethnicity, Community and Nature of Group Conflict in Mauritius », http://arinave.com/wp-content/uploads/2012/05/NestedIdentities.pdf, consulté le 28 férvrier 2014.

Neewoor, Anand, «India-Mauritius Relations », Mother India. Children Abroad, 2, 1988, p. 27-30.

Nevin, Andrew, «Pimpology 101. An Examination of the Pimp as an Acceptable Social Role in a Capitalist Society », Sociological Imagination: Western's Undergraduate Sociology Student Journal, 2, 2013, p. 1-18.

Oodiah, Malenn, D., « Communalisme / Ethnicité : théories, concepts, notions, analyses. Revue critique et nouvelles perspectives », Journal of Mauritian Studies, 2, 1998, p. 87-134.

Office of the United Nations Resident Coordinator, Mauritius, «Common Country Assessment.Mauritius », http://www.law.yale.edu/rcw/rcw/jurisdictions/afe/mauritius/mauritius assessment.pdf, consulté le 3 septembre 2013.

Office of Public Communication, Bureau of Public Affairs, « Historical, Political and Economic Overviews of the Countries of the World.Mauritius », http://dosfan.lib.uic.edu/ERC/bgnotes/af/mauritius9211.html, consulté le 23 octobre 2012.

Polselli, Marine, « Ponctionlombaire.com », 2010, http://www.ponctionlombaire.com/, consulté le 13 mars 2012.

Port-Louis City Landslide Project, http://www.jica.go.jp/english/our_work/evaluation/oda_loan/post/2002/pdf/136 full.pdf, consulté le 3 septembre 2013.

Quinn, Eithne, « Who's the Mack?: the Performativity and Politics of the Pimp Figure in Gangsta Rap », Journal of American Studies, 34, 2000, p. 115-136. 
Rajah-Carim, Aaliya, «Language Use and Attitude in Mauritius on the Basis of the 2000 Population Census », Journal of Multilingual and Multicultural Development, 26, 2005, p. 317-332.

Ramdhony, Raj, « Mauritius. An Overview », Mother India. Children Abroad, 2, 1988, p. 1-8.

Ramdhony, Reshmi, «Future of Mauritian Women in the New Economic Order », Mother India. Children Abroad, 2, 1988, p. 77-98.

Ramdoyal, Ramesh, L'île Maurice à travers ses fêtes, Singapour, Singapour National Printers, 1993.

Ramharai, Vicram, «Entre littérature mauricienne et littérature francophone : quels enjeux pour les écrivains mauriciens ? », Postcolonial Literatures of the Francophone Indian Ocean, 2, 2008, http://www.reading.ac.uk/efrance/Indian\%200cean/Ramharai.pdf, consulté le 5 septembre 2011.

Ramsoondur-Mungur, Angela, «Female Indo-Mauritian Negotiation of Beauty and Bollywood TV Serials », https://www.academia.edu/993399/Female_IndoMauritian_negotiation_of_beauty_and_Bollywood_TV_serials_draft_paper_, consulté le 10 février 2014.

Raphael, Jody, Brenda Myers-Powell, « From Victims to Victimizers : Interviews with 25 Ex-Pimps in Chicago », http://newsroom.depaul.edu/pdf/family law center report-final.pdf, consulté le 23 janvier 2014.

Raub, Werner, Vincent Buskens, Marcel Van Assen, « Micro-Macro Links and Microfoundations in Sociology », 35, 2011, p. 1-25.

Ravi, Srilata, «Indo-Mauritians: National and Postnational Identities », L'Esprit créateur, 50, 2010, p. 29-45.

Ravi, Srilata, «Cultivating Indianness: the Indian Labour in Mauritian Imagery », $e$ France: an on-line Journal of French Studies, 2, 2008, p. 116-131.

Rivière, Lindsay, Historical Dictionary of Mauritius, London, The Scarecrow Press, 1982.

Rothstein, Edward, «Claude Lévi-Strauss, 100, Dies. Altered Western Views of the "Primitive" ", http://www.nytimes.com/2009/11/04/world/europe/04levistrauss.html?pagewan ted=1\& r=0\&em, consulté le 12 décembre 2013.

Romenesko, Kim, Eleanor Miller, « The Second Step in Double Jeopardy: Appropriating the Labor of Female Street Hustlers », Crime and Delinquency, 35, 1989, p. 109-135.

Runkle, Susan, «The Beauty Obsession. International Standards for Fair and Lovely », http://www.manushi-india.org/pdfs_issues/PDF\%20145/145\%2011-16.pdf, consulté le 10 février 2014.

SADC Gender Protocol, « Mauritius. Homophobia: One Step Forward, Two Steps Back », http://www.genderlinks.org.za/article/mauritius---homophobia-onestep-forward-two-steps-back-2011-06-17, consulté le 25 mars 2013. 
Schreiber, Andrea, «Alcoholism », Oral Surgery Oral Medicine Oral Pathology Oral Radiology and Endodontology, 92, 2001, p. 127-131.

Serughetti, Giorgia, « Prostitution and Clients' Responsibility », Men and Masculinities, 16, 2012, p. 35-48.

Shankar R., P. Subish, « Fair Skin in South Asia: an Obsession? », Journal of Pakistan Association of Dermatologists, 17, 2007, p. 101-105.

Simone, Abdou Maliq, «On the Dynamics of Ambivalent Urbanization and Urban Productivity in Port Louis, Mauritius » dans Small Cities. Urban Experience beyond the Metropolis, Oxon, Routledge, 2006, p. 21-32.

Simmons, Adele, Modern Mauritius. The Politics of Decolonization, Bloomington, Indiana University Press, 1982.

Special Mobile Force, « Presentation of Regimental Colour », Government Printing, 2010.

Spidel A., C. Greaves, B. Cooper, H. Hervé, R. Hare, J. Yuille, « The Psychopath as Pimp », The Canadian Journal of Police and Security Services, 4, 2006, p. 193199.

Srebrnik, Henry, « Can an Ethnically-Based Civil Society Succeed? The Case of Mauritius », Journal of Contemporary African Studies, 18, 2000, p. 7-20.

Suntoo, Rajen, Hemant Chittoo, « Managing Multi-Ethnic Mauritius », Global Journal of Human Social Science, 12, 2012, p. 33-41.

The Pretoria News, «Commission Addressed Dark History of Mauritian Slavery », http://search.proquest.com/docview/912095263?accountid=14782, consulté le 30 octobre 2013.

ThinkQuest, « Social Stratification in Mauritius », http://library.thinkquest.org/C0110237/Population/Social Classification/social classification.html, consulté le 13 janvier 2014.

Thompson, Cheryl, « Black Women and Identity: What's Hair Got to Do with It ? », Politics and Performativity, 22, 2008- 2009, p. 78-89.

Titmuss, Richard, Social Policies and Population Growth in Mauritius, London, Eyre and Spottiswoode, 1961.

Toorawa, Shawkat, «Is Multiculturalism Bad for Art? Carl de Souza's La maison qui marchait vers le large and the Mauritian City », L'Esprit créateur, 41, 2001, p. 197-206.

Toolsee, Damodur, «Legal System in Mauritius », Mother India. Children Abroad, 2, 1988, p. 42-46.

Toussaint, Auguste, Port-Louis. A Tropical City, Londres, Allen and Unwin, 1973.

Treilles, Johanna, « Sourcière ou Sorcière ? Archétypes et représentations des mères dans les écrits contemporains des îles de l'océan Indien », communication présentée à la conférence organisé par APFUCC, 27-31 mai 2011.

Treisman, Daniel, « The Causes of Corruption: a Cross-National Study », Journal of Public Economics, 73, 2000, p. 399-457.

Tsekeris, Charalambos, Anna Lydaki, « The Micro-Macro Dilemma in Sociology:Perplexities and Perspectives », 3, 2011, p. 67-82. 
Valere, Melanie, « Dossier "Femmes". Les Humains s'accordent aussi au féminin », http://www.defimedia.info/defi-quotidien/dq-societe/item/49252-dossierfemmes-les-droits-humains-s-accordent-aussi-au-feminin.html, consulté le 21 mars 2014.

Van Gee, Susan, «Alcoholism and the Family: a Psychodrama Approach », Journal of Psychiatric Nursing and Mental Health Services, 17, 1979, p. 9-12.

World Health Organization, « Global and Regional Estimates of Violence against Women: Prevalence and Health Effects of Intimate Partner Violence and NonPartner Sexual Violence. $2013 »$, http://apps.who.int/iris/bitstream/10665/85239/1/9789241564625 eng.pdf, consulté le 21 mars 2014.

Wilson, Jason, Bernardin de Saint-Pierre. Journey to Mauritius, Oxford, Signal Books, 2002.

Williamson, Celia, Terry Cluse-Tolar, « Pimp-Controlled Prostitution », Violence Against Women, 8, 2002, p. 1074-1092.

Winick, Charles, «Prostitutes' Clients' Perception of the Prostitutes and of Themselves », International Journal of Social Psychiatry, 8, 1962, p. 289-296.

Wolfgang, Lutz, «Spotlight. Mauritius », Population Today, 25, 1997, p. 7.

Zafar, Ali, «Mauritius: an Economic Success Story », http://siteresources.worldbank.org/AFRICAEXT/Resources/Mauritius_success.p df, consulté le 14 novembre 2013. 